Giovanni Placini

\title{
On the Geometry and Topology of Sasakian Manifolds
}

Dissertation an der

Fakultät für Mathematik, Informatik und Statistik der Ludwig-Maximilians-Universität München

Vorgelegt am

29. Juni 2020

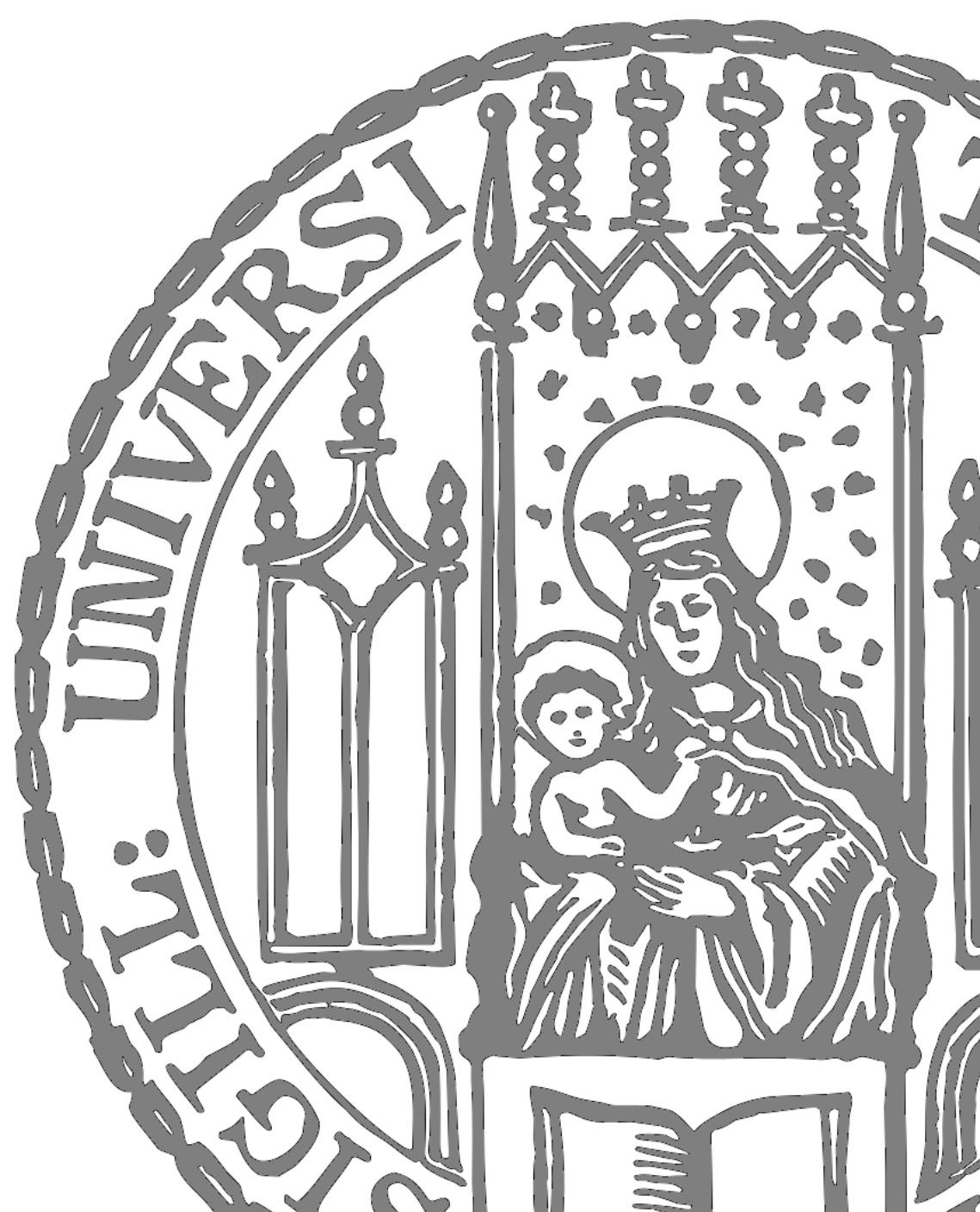




\section{Erster Gutachter}

Prof. Dieter Kotschick, D. Phil. (Oxon)

(Ludwig-Maximilians-Universität München)

\section{Zweiter Gutachter}

Prof. Dr. Beniamino Cappelletti Montano

(Università di Cagliari)

\section{Dritter Gutachter}

Prof. Dr. Oliver Goertsches

(Philipps-Universität Marburg)

Tag der Disputation: 04.08.2020 


\begin{abstract}
This thesis is concerned with the topology and geometry of Sasakian manifolds. Sasaki structures consist of certain contact forms equipped with special Riemannian metrics. Sasakian manifolds relate to arbitrary contact manifolds as Kählerian or projective complex manifolds relate to arbitrary symplectic manifolds. Therefore, Sasakian manifolds are the odd-dimensional analogs of Kähler manifolds.

In the first part of the thesis we discuss some geometric invariants of Sasaki structures. Specifically, the socalled basic Hodge numbers, the type and their relation to the underlying contact and almost contact structures are discussed. We produce many pairs of negative Sasakian structures with distinct basic Hodge numbers on the same differentiable manifold in any odd dimension larger than 3.

In the second part of the thesis we discuss topological properties of Sasakian manifolds, focussing particularly on the fundamental groups of compact Sasakian manifolds. In parallel with the theory of Kähler and projective groups, we call these groups Sasaki groups. We prove that any projective group is realizable as the fundamental group of a compact Sasakian manifold in every odd dimension larger than three. Similarly, every finitely presentable group is realizable as the fundamental group of a compact K-contact manifold in every odd dimension larger than three. Nevertheless, Sasaki groups satisfy some very strong constraints, some of which are reminiscent of known constraints on Kähler groups. We show that the class of Sasaki groups is not closed under direct products and that there exist Sasaki groups that cannot be realized in arbitrarily large dimension. We prove that Sasaki groups behave similarly to Kähler groups regarding their relation to 3-manifold groups and to free products.
\end{abstract}

\title{
Zusammenfassung
}

Thema der vorliegenden Arbeit ist die Topologie und Geometrie von Sasakimannigfaltigkeiten. Eine Sasaki Struktur setzt sich aus einer gewissen Kontaktform und einer speziellen Riemannschen Metrik zusammen. Sasaki Mannigfaltigkeiten verhalten sich zu Kontaktmannigfaltigkeiten wie Kählermannigfaltigkeiten oder projektive komplexe Mannigfaltigkeiten zu beliebigen symplektischen Mannigfaltigkeiten. Daher sind Sasaki Mannigfaltigkeiten als das ungerade dimensionale Analogon von Kählermannigfaltigkeiten anzusehen.

Im ersten Teil der Arbeit diskutieren wir einige geometrische Invarianten von Sasaki Strukturen. Genauer behandeln wir die sogenannten basischen Hodge Zahlen, den Typ und den Zusammenhang mit den unterliegenden Kontakt- und Fastkontaktstrukturen. Wir konstruieren, in jeder ungeraden Dimension größer als 3, viele Beispiele von Paaren negativer Sasaki Strukturen mit verschiedenen basischen Hodge Zahlen auf derselben differenzierbaren Mannigfaltigkeit.

Im zweiten Teil der Arbeit diskutieren wir topologische Eigenschaften von Sasaki Mannigfaltigkeiten, wobei der Fokus auf den Fundamentalgruppen kompakter Sasaki 
Mannigfaltigkeiten liegt. Parallel zur Theorie der Kählergruppen und projektiven Gruppen nennen wir solche Gruppen Sasakigruppen. Wir zeigen, dass sich jede projektive Gruppe als Fundamentalgruppe einer kompakten Sasakimannigfaltigkeit in jeder ungeraden Dimension größer als drei realisieren lässt. Analog lässt sich jede endlich präsentierbare Gruppe als Fundamentalgruppe einer K-Kontaktmannigfaltigkeit in jeder ungeraden Dimension größer als drei realisieren. Nichtsdestotrotz unterliegen Sasakigruppen einigen sehr starken Einschränkungen, von denen manche an bekannte Einschränkungen für Kählergruppen erinnern. Wir zeigen dass die Klasse der Sasakigruppen nicht unter direkten Produkten abgeschlossen ist und dass es Sasakigruppen gibt die nicht in beliebig großer Dimension realisiert werden können. Wir beweisen dass sich Sasakigruppen bezüglich ihrer Beziehung zu 3-Mannigfaltigkeitsgruppen und freien Produkten ähnlich wie Kählergruppen verhalten. 




\section{Contents}

$\begin{array}{lll}1 & \text { Introduction } & 1\end{array}$

1.1 Invariants of Sasaki structures . . . . . . . . . . . . . 2

1.2 Fundamental groups of compact Sasakian manifolds . . . . . . . . 5

1.3 Organization of the thesis $\ldots \ldots \ldots \ldots 7$

\begin{tabular}{|lll}
\hline 2 & Orbifolds & 9
\end{tabular}

2.1 Definitions . . . . . . . . . . . . . . . . . . . . . . . . 9

2.2 Orbibundles . . . . . . . . . . . . . . . . . . . . 11

2.3 Orbifold classifying spaces . . . . . . . . . . . . . . . . . 13

2.4 Complex cyclic orbifolds . . . . . . . . . . . . . . . . 20

\begin{tabular}{llr}
\hline & Sasaki manifolds & 27
\end{tabular}

3.1 Contact and almost contact structures . . . . . . . . . . . . . 27

3.2 The Boothby-Wang fibration . . . . . . . . . . . . . . . 33

3.3 K-contact structures . . . . . . . . . . . . . . . . . . . 35

3.4 Complex structures on the Riemannian cone . . . . . . . . . . . . . . . . . . . 40

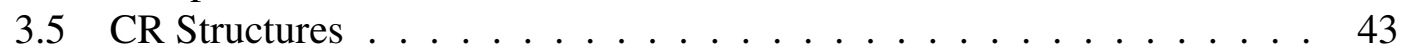

3.6 Sasaki structures $\ldots \ldots \ldots \ldots$. . . . . . . . . . . . . . . . 44

3.7 Transverse Hodge theory . . . . . . . . . . . . . . . . . . 55

3.8 The join construction $\ldots \ldots \ldots \ldots$. . . . . . . . . . . . . . 61

3.9 Topology of Sasakian manifolds $\ldots \ldots$. . . . . . . . . . . 63

$\begin{array}{|lll|}4 & \text { Group extensions and Kähler groups } & 67\end{array}$

4.1 Group cohomology and central extensions . . . . . . . . . . . . . . 67

4.2 Kähler groups $\ldots \ldots \ldots \ldots$. . . . . . . . . . . . . . . . . . . . . . . . . . . . . . . 70

4.3 Analogues in the Sasakian setting . . . . . . . . . . . . 75

\begin{tabular}{|lll}
\hline 5 & Invariants and underlying structures & $\mathbf{7 9}$
\end{tabular}

5.1 Invariance of basic Betti and Hodge numbers . . . . . . . . . . . 79

5.2 Complete intersections . . . . . . . . . . . . . . . 81

$5.3 \quad$ Distinguished Sasaki structures on a smooth manifold . . . . . . . . . . 83

5.3 .1 Simply connected 5-manifold . . . . . . . . . 83 
5.3 .2 Simply connected 7-manifolds . . . . . . . . . . . . . 90

5.3 .3 Simply connected higher dimensional manifolds . . . . . . . 93

$\begin{array}{lll}6 & \text { Sasaki groups } & 95\end{array}$

6.1 The short exact sequence of a quasi-regular Sasaki structure . . . . . . 95

6.2 Realizability . . . . . . . . . . . . . . . . . . . . . . . 99

6.3 Direct products . . . . . . . . . . . . . . . . . . . . . . . 103

6.4 Free products . . . . . . . . . . . . . . . . . . . . . . . . . 104

6.5 Three-manifold groups $\ldots \ldots$. . . . . . . . . . . . . . . 106

6.6 Further consequences . . . . . . . . . . . . . . . . . . . 108 


\section{Chapter 1}

\section{Introduction}

Sasaki structures were introduced by Sasaki and Hatakeyama in [103] building on previous work of Sasaki [102]. Unfortunately the study of Sasaki manifolds, although very active, remained confined to Japan for the following years. The first attempt to spread the interest for the subject was made by Blair [8]. Nevertheless, the study of Sasakian geometry did not meet the enthusiasm reserved for its even dimensional analogue, Kähler geometry. In fact, Sasakian geometry was not seen as the multifaceted subject it is but rather considered as a subfield of Riemannian geometry. Sasakian geometry and topology met renewed interest after the work of Boyer and Galicki and, specifically, the publication of their seminal book [15]. In [15] Boyer and Galicki give an account of the interplay between Sasakian and complex algebraic geometry as well as differential topology, albeit the main focus of the book remains on Sasaki-Einstein metrics. This initiated an intensive study of Sasakian geometry and topology through the interrelation with other geometries.

Sasaki structures are contact structures with a special transverse complex structure. As such, they are related to symplectic and Kähler manifolds as follows. A contact manifold $(M, \eta)$ is a smooth $(2 n+1)$-dimensional manifold $M$ endowed with a contact form $\eta$, i.e. a 1 -form such that $\eta \wedge \mathrm{d} \eta^{n} \neq 0$. It is easy to check that the cone over a contact manifold $C=M \times \mathbb{R}^{+}$carries the symplectic form $\Omega=\mathrm{d}(t \eta)$. Moreover, the contact form $\eta$ defines the Reeb foliation $\mathcal{F}$, that is, the foliation given by the orbits of the Reeb vector field $R$. The contact distribution $\mathcal{D}=\operatorname{ker} \eta$ is endowed with the symplectic structure $\mathrm{d} \eta$. Therefore the transverse space $X$ to the Reeb foliation is naturally symplectic. The existence of an almost complex structure $J$ on $X$ compatible with the symplectic form is equivalent to the existence of an almost complex structure $\widetilde{J}$ on the contact distribution $\mathcal{D}$ compatible with $\mathrm{d} \eta$ which is preserved by the Reeb vector field, that is, such that $\mathcal{L}_{R} \widetilde{J}=0$. In fact, this is a one-to-one correspondence. A contact manifold endowed with such an almost complex structure is a K-contact manifold. The cone over a K-contact manifold is naturally equipped with an almost complex structure I compatible with $\mathrm{d}(t \eta)$ and hence is itself an almost Kähler manifold. This is the relation between contact and symplectic structures or, more precisely, between K-contact 
and almost Kähler manifolds.

A Sasaki manifold is a K-contact manifold whose cone $\left(M \times \mathbb{R}^{+}, \mathrm{d}(t \eta), I\right)$ is Kähler. This condition is equivalent to the space $X$ being Kähler. That is, every Sasaki manifold can be viewed as a manifold endowed with a contact form $\eta$ whose Reeb foliation is transversally Kähler. Therefore, Sasaki structures can be thought of as the odd dimensional analogue of Kähler structures. This description highlights the fact that Sasaki manifolds relate to K-contact manifolds as Kähler manifolds relate to almost Kähler manifolds.

We should mention that Sasaki structures are more often defined as contact structures with a special Riemannian metric $g$. This is equivalent to our approach. In fact, the metric $g$ is determined unequivocally by the almost complex structure and vice versa. This reflects the fact that in an almost Kähler triple $(\omega, J, h)$ on $X$ the metric $h$ determines $J$ and vice versa.

When the foliation $\mathcal{F}$ is regular we have a socalled Boothby-Wang fibration. That is, a principal $S^{1}$-bundle

$$
\pi:(M, \eta) \longrightarrow(X, \omega)
$$

where $(X, \omega)$ is a smooth Kähler manifold and $\pi^{*} \omega=\mathrm{d} \eta$. Furthermore, $[\omega]$ is the first Chern class of the bundle and $X$ is a smooth projective variety. This description is closer to the general case than one might suspect. In fact, every Sasakian manifold is a principal $S^{1}$-bundle over a projective orbifold obtained from a quasi-regular Sasaki structure, cf. the Structure Theorem 3.59

Given the multitude of underlying structures it is evident that Sasakian geometry can be studied from several viewpoints implementing tools from various other geometries. In this thesis we will focus on contact and almost contact geometry as well as Kähler geometry. The study of Sasakian geometry and topology in this thesis can be divided in two parts. The first one investigates transverse invariants of Sasaki structures while the latter investigates fundamental groups of compact Sasakian manifolds.

In this thesis all manifolds are understood to be smooth, closed, connected and orientable unless otherwise stated.

\subsection{Invariants of Sasaki structures}

In the first part of this thesis we study some invariants of Sasaki structures and discuss their dependence on the underlying contact and almost contact structures. In particular, we focus on the type of Sasaki structures and their basic Hodge numbers and relate this to the topology of almost contact and contact structures.

Contact structures are maximally non-integrable hyperplane distributions on a manifold of dimension $2 n+1$. Eliashberg [39] introduced a dichotomy of 3-dimensional contact structures into overtwisted and tight ones. The definition of overtwisted contact structure, hence the dichotomy of contact structures, was then extended to higher dimensions in [12]. The contrast between overtwisted and tight structures is encountered, 
for instance, when considering symplectic fillability. A (strong) symplectic filling of a closed contact manifold $(M, \eta)$ is a symplectic manifold $(W, \Omega)$ with $\partial W=M$ such that $\Omega=\mathrm{d} \alpha$ near the boundary, $\mathcal{D}=\operatorname{ker} \eta=\operatorname{ker} \alpha_{\left.\right|_{T M}}$ and $\mathrm{d} \alpha_{\mid \mathcal{D}}>0$. Both in dimension three and in higher dimensions the existence of a symplectic filling gives an obstruction to overtwistedness, that is, a symplectically fillable contact manifold is tight [40, 93]. As mentioned above, a regular Sasaki manifold $(M, \eta)$ is a principal $S^{1}$-bundle over a projective manifold. The disc bundle given by filling the fibers of the Boothby-Wang fibration is a symplectic filling of $(M, \eta)$. As a consequence of a theorem of Niederkrüger and Pasquotto [94] on resolutions of cyclic orbifold singularities, the same result holds for quasi-regular K-contact, hence Sasaki, structures. Therefore, any quasi-regular Sasaki structure is tight as a contact structure.

In [39] Eliashberg proved a parametric $h$-principle for overtwisted contact structures. Namely, any homotopy class of almost contact structures on a closed 3-manifold contains a unique isotopy class of overtwisted contact structures. This classification was later extended to higher dimensions by Borman, Eliashberg and Murphy [12]. By contrast, the topology of tight contact structures is less understood than that of overtwisted contact structures and is not necessarily given by an $h$-principle. For instance, two overtwisted contact structures with homotopic underlying almost contact structures are isotopic as contact structures. This is not necessarily the case for Sasaki structure because their contact structures are always tight. Therefore, two Sasaki structures on the same manifold can have equivalent almost contact structures but inequivalent contact structures. We discuss instances of this phenomenon in Chapter 5. In particular, we prove that the fact that an almost contact structure supports a Sasaki structure does not give a bound on the number of isotopy classes of tight contact structures in its homotopy class.

Theorem 5.20. For all positive integers $k>0$ there exists a simply connected 5manifold admitting $k$ Sasaki structures with homotopic almost contact structures but pairwise inequivalent contact structures.

Basic Hodge numbers are transverse invariants of the Reeb foliation. As such, they depend, a priori, on the Reeb vector field and the complex bundle $(\mathcal{D}, J)$. The transverse geometry of the Reeb foliation of a Sasaki structure is very rich. Cohomological properties of the transverse space $X$, that is, the socalled basic cohomology of the foliation, were studied by El Kacimi-Alaoui, Hector and Nicolau [41, 42, 43]. They proved that the basic cohomology of a Sasaki manifold shares many properties with the cohomology of Kähler manifolds. For instance, one can define a basic Dolbeault double complex and prove that it satisfies the Hodge decomposition Theorem and Poincaré and Serre duality. This leads to the definition of basic Betti and Hodge numbers $\mathrm{b}_{B}^{r}(\mathcal{F})$ and $h_{B}^{p, q}(\mathcal{F})$. While the former turn out to be topological invariants [15, Theorem 7.4.14], the latter do not depend only on the underlying smooth manifold. However, in [49] it is proved that basic Hodge numbers are invariant under CR deformations of the Sasaki structure. Recently Raźny [100] showed that basic Hodge numbers are invariant under 
arbitrary smooth deformations of Sasaki structures. Moreover, in [49] is provided a 5dimensional manifold endowed with two Sasaki structures with differing basic Hodge numbers, see Example 5.3. To the best of the author's knowledge this was the only known example of Sasaki structures with different basic Hodge numbers on the same smooth manifold before this thesis.

Basic Hodge numbers are closely related to another transverse invariant, namely, the type of the Sasaki structure. In parallel with the standard case one can define basic Chern classes $c_{i}(\mathcal{F})$ with the use of a a transverse connection. Again many properties that are encountered on Kähler manifolds are satisfied. For instance, El Kacimi-Alaoui [41] showed that if $2 \pi c_{1}(\mathcal{F})$ is represented by a real basic $(1,1)$-form $\rho$, then it is the Ricci curvature of a unique transverse Kähler form $\omega$ with $[\omega]=[\mathrm{d} \eta]$. It is then natural to call a Sasaki structure of positive, respectively negative type or null, if $c_{1}(\mathcal{F})$ can be represented by a positive definite, resp. negative definite or null, $(1,1)$-form. An instance of the relation between the type and basic Hodge numbers is the transverse vanishing theorem proven independently by Goto and Nozawa, see [51, 95]. Namely, if a Sasaki structure is positive, its basic Hodge numbers $h_{B}^{p, 0}(\mathcal{F})$ vanish for all $p>0$.

The Sasaki structures with different basic Hodge numbers given in [49] are of different type. Specifically, one of the structures is positive while the other is null. This motivates our study of manifolds admitting Sasaki structures with different basic Hodge numbers. We construct many Sasaki structures of the same type whose basic Hodge numbers disagree. Namely, we prove the following:

Theorem 5.21. There exist infinitely many simply connected 5-manifolds admitting two negative Sasaki structures whose basic Hodge numbers disagree. Moreover, these pairs of Sasaki structures have homotopic underlying almost contact structures but inequivalent contact structures.

These results on 5-manifolds rely on Barden's classification of simply connected 5manifolds [7], Geiges's classification of almost contact structures on simply connected 5-manifolds [47] and a result of Hamilton's [59] on the equivalence classes of almost contact and contact structures in dimension 5. No similar results exist in higher dimension. In order to generalize Theorem 5.21 to higher dimensions we turn our attention to complete intersections. These projective varieties have a rather simple cohomology that allows us to control the geometry of Boothby-Wang bundles. Namely, appealing to Wall's classification of simply connected 6-manifolds and the Hirzebruch-RiemannRoch Theorem, we extend Theorem 5.21 to dimension 7 albeit in the following weaker form:

Theorem 5.25. There exist infinitely many 7-manifolds admitting two negative Sasaki structures with different basic Hodge numbers. Moreover, these manifolds can be arranged to be spin or non-spin.

We conclude the study of transverse invariants generalizing the result above to any dimension. 
Theorem 5.28, For all $n>1$ there exist countably many $(2 n+1)$-dimensional manifolds admitting two Sasaki structures with different basic Hodge numbers. Moreover, these manifolds can be arranged to be spin or non-spin and one can pick the Sasaki structures to be negative or indefinite.

\subsection{Fundamental groups of compact Sasakian manifolds}

It is a classical problem to determine the relations between geometry and topology or, more specifically, whether a certain geometric property imposes constraints on the topology of a manifold. A standard instance of this interplay between topology and geometry is represented by the restriction that positive curvature properties prescribe on the topology of a manifold. We focus on a specific case of the problem above. Namely, we are interested in the following:

Question: Which restrictions on the fundamental group of a compact manifold are given by the existence of a certain geometric structure?

A well known corollary of Hodge theory states that odd degree Betti numbers of Kähler manifolds are even. In particular, so is the first Betti number of a Kähler manifold, hence the rank of the abelianization of its fundamental group. This type of observation justifies the interest for the class of fundamental groups of compact Kähler manifolds as well as symplectic and complex manifolds. Kotschick proved in [75] that every finitely presentable group is the fundamental group of a 4-dimensional almost complex manifold and asked whether the same holds for symplectic manifolds. Building on a theorem of Taubes [112] Carlson and Kotschick independently noted that every finitely presentable group is the fundamental group of a compact complex 3-fold. Shortly after, Gompf [50] proved an analogous statement for 4-dimensional symplectic manifolds. Moreover, he observed that the complex 3-fold in [112] can be arranged to be simultaneously symplectic.

Therefore, a Kähler structure imposes restrictions on the fundamental group while complex or symplectic structures do not. Given the analogy between Kähler and Sasakian geometry it is natural to ask whether or not the same is true for Sasaki structures. The answer to this question is affirmative. It was proven in [76] that the existence of an almost contact structure does not impose any restriction on the fundamental group. We will see later that the same holds for K-contact structures. Moreover, it was pointed out already in [15] that the results of El Kacimi-Alaoui [41] imply that the first Betti number of a Sasakian manifold is even.

In light of this, in the second part of the thesis we focus on properties of fundamental groups of compact Sasakian manifolds. In analogy with the Kähler case we will call these groups Sasaki groups. Kähler groups have been an active field of research in the last 30 years with important contributions from many mathematicians, see [2] for an 
introduction. In comparison, very little is known about Sasaki groups, see however [10, 30, 68].

Firstly we investigate the realizability problem for Sasaki groups. Namely, we ask which groups can be realized as fundamental groups of compact Sasakian manifolds and in which dimension. Three-dimensional Sasaki and K-contact manifolds were classified by Geiges [48]. As a consequence their fundamental groups are lattices in $S O(4)$, $S \widetilde{L(2, \mathbb{R})}$ or the real Heisenberg group $\mathcal{H}_{3}$. This provides a solution to the problem of realizability in dimension 3. Chen showed in [30] that every projective group can be realized as the fundamental group of a compact Sasakian manifold of any odd dimension $2 n+1 \geqslant 7$. More recently a similar statement was proven [10] for K-contact manifolds. Namely, every finitely presentable group can be realized as the fundamental group of a compact K-contact manifold of any odd dimension $2 n+1 \geqslant 7$. Our first results on Sasaki and K-contact groups sharpen these results:

Theorem 6.5. Every projective group $\Gamma$ can be realized as the fundamental group of a compact Sasakian manifold of any odd dimension $2 n+1 \geqslant 5$.

Theorem 6.7. Every finitely presentable group $\Delta$ can be realized as the fundamental group of a compact $K$-contact manifold of any odd dimension $2 n+1 \geqslant 5$.

We investigate further the problem of realizability by asking in which dimension a given Sasaki group can be realized. In the projective setting the problem is completely solved by the classification of Riemann surfaces, the Lefschetz Hyperplane theorem and the following observation. It is enough to take the Cartesian product with a suitable number of copies of $\mathbb{C P}^{1}$ to realize a Kähler or projective group in arbitrarily large dimensions. We show that this is not the case for Sasaki groups. Namely we prove the following

Theorem 6.12, For $n=1,2,3$ there exist $(2 n+1)$-dimensional Sasakian manifolds whose fundamental group cannot be realized by a Sasakian manifold of higher dimension.

We believe this result to be true in any dimension $2 n+1$ but we were not able to prove it.

Another rather elementary property of Kähler groups is closedness under direct products. In fact, the product of two Kähler manifold is again Kähler. Thus the direct product of two Kähler groups is a Kähler group. Products of Sasakian manifolds are not Sasakian for dimension reasons. A natural approach is to try to perform a construction, e.g. a join construction, while controlling the fundamental group. We prove in Section 6.3 that this is not possible:

Theorem 6.15. The set $\mathcal{S}$ of Sasaki groups is not closed under direct products.

Theorem 6.12 and Theorem 6.15 show that the class of Sasaki groups is an interesting research subject in its own right. However some of the constraints satisfied by 
Sasaki groups are reminiscent of the restrictions that Kähler groups are subject to. In fact, in some instances Sasaki and Kähler groups present very similar behaviour.

It is easy to see that free groups cannot be Sasaki. Motivated by this observation we investigate the case of free products. Namely, we prove that under mild hypotheses on $\Gamma_{1}$ and $\Gamma_{2}$ the group $\left(\Gamma_{1} * \Gamma_{2}\right) \times H$ is not Sasaki for any group $H$. This shows in particular that such Sasaki groups are indecomposable under free products whose factors have non-trivial finite quotients. Namely, we prove the Sasakian analogue of a theorem of Johnson and Rees [66] for Kähler groups.

Theorem 6.18, Let $\Gamma_{1}$ and $\Gamma_{2}$ be two groups. Assume $f_{i}: \Gamma_{i} \longrightarrow Q_{i}$ is a non-trivial quotient with with $\left|Q_{i}\right|=m_{i}<\infty$ for $i=1,2$.

a) Then $\left(\Gamma_{1} * \Gamma_{2}\right)$ is not Sasaki.

b) For any group $H$ the product $\left(\Gamma_{1} * \Gamma_{2}\right) \times H$ is not Sasaki.

A well studied class of groups is that of 3-manifold groups, i.e. fundamental groups of (not necessarily closed) manifolds of dimension 3. These groups enjoy different properties than Kähler groups. For instance, by taking connected sums one sees that 3 manifold groups are closed under free products. Motivated by the results on free groups and free products we discuss the relation between Sasaki and 3-manifold groups. It was proved in [79] that if a 3-manifold group is also a Kähler group, then it is a surface group or a finite group. Clearly the analogous statement in the Sasaki setting should involve only Sasaki groups realizable in dimension 5 or higher. It turns out that Sasaki groups have very little in common with 3-manifold groups, apart from the obvious intersection given by surface groups and finite groups.

Theorem 6.21, Let $\Gamma$ be an infinite 3-manifold group. Then $\Gamma$ is the fundamental group of a Sasakian manifold of dimension $2 n+1 \geqslant 5$ if and only if $\Gamma$ is the fundamental group of a closed orientable surface.

As an immediate consequence of Theorem 6.21 we get a dichotomy of infinite Sasaki groups into those realizable in dimension 3 and all others.

Corollary 6.23. The fundamental group of a three-dimensional Sasakian manifold is realizable in higher dimensions if and only if it is finite.

\subsection{Organization of the thesis}

In Chapter 2 we give an overview of orbifolds. Section 2.1 and Section 2.2 focus on the definitions of orbifolds and of related concepts such as orbibundles, metrics and forms. In Section 2.3 we turn our attention to orbifold classifying spaces. This allows us to introduce orbifold invariants such as orbifold cohomology and orbifold fundamental groups which play a central role in this thesis. In the last section of Chapter 2 we discuss complex orbifolds with a particular focus on Seifert bundles. 
Chapter 3 is devoted to Sasaki manifolds and their topological and geometric invariants. In the first part of the chapter we introduce almost contact and contact structures. We then discuss CR structures and the metric cone in order to define K-contact and Sasaki manifolds. In Section 3.6 we define Sasaki structures and discuss the various approaches that can be taken. Moreover, we prove the Structure Theorem 3.59 [15. Theorem 7.1.3 and Theorem 7.1.6] that will play an essential role in the remainder of this thesis. Next we present the join construction. This is, roughly speaking, the analogue of Cartesian products in the Sasaki setting. Section 3.7 is dedicated to the transverse geometry of K-contact and Sasaki manifolds. Several geometric invariants of Sasaki manifolds are defined here making this section crucial for the results proven in Chapter 5. The last section of Chapter 3 gives an overview of topological properties of Sasakian manifolds. Due to its importance the role of Boothby-Wang fibrations is stressed throughout the chapter and their treatment is a constant focus.

Chapter 4 serves as motivation for the study of Sasaki groups carried in Chapter 6 . In Section 4.1 we recall some basic notions on group cohomology and central extensions. We then proceed to present some results on Kähler groups in Section 4.2. This section gives a (by no means exhaustive) treatment of our current understanding of Kähler groups. We conclude Chapter 4 with a review of the properties of Sasaki groups proven in [10, 23, 30, 68].

Chapter 5 is dedicated to the proofs of the results presented in Section 1.1 above. In Section 5.1 we discuss invariance of basic Betti and Hodge numbers. This serves as motivation for the results in Section 5.3. The proof of these results is preceded by a review of the geometry of complete intersections carried out in Section 5.2. Particular attention is reserved to the Hodge and Chern numbers of these projective varieties.

Chapter 6 is dedicated to the study of Sasaki groups. First we give a correspondence between quasi-regular Sasaki structures and the fundamental group as central extensions in Section 6.1. The remainder of the chapter is devoted to the proofs of the results exposed in Section 1.2 above.

\section{Acknowledgements}

I am indebted to my supervisor Prof. D. Kotschick for the patience, guidance, the enlightening discussions and the many ideas that he shared with me. I would like to thank R. Coelho, N. Pia and J. Stelzig for being always available for helpful discussions which were source of never trivial comments and suggestions. Finally, I am grateful to all the members of the differential geometry and topology group for the lively and stimulating environment. 


\section{Chapter 2}

\section{Orbifolds}

Orbifolds play a fundamental role in the study of Sasakian topology and geometry. This chapter is dedicated to orbibundles and their classification as well as topological invariants of orbifolds.

Riemannian orbifolds were introduced and studied by Satake in [104] and [105] under the name of V-manifolds. Independently, Baily introduced complex orbifolds and proved the Hodge decomposition [5] and Kodaira's Embedding Theorem [6] in this setting. Most of the material of this chapter can be found in [15, Chapter 4] and in the book [1] which provides an excellent introduction to orbifolds and their relation to groupoids. Omitted proofs in this chapter can be found in [1, 15] and references therein.

\subsection{Definitions}

Let us start by defining the central object of this chapter.

Definition 2.1. Let $X$ be a topological space and fix $n \geqslant 0$.

1) An $n$-dimensional orbifold chart (or uniformizing chart) $(\widetilde{U}, \Gamma, \varphi)$ on $X$ is given by an open connected set $\widetilde{U} \subset \mathbb{R}^{n}$, a finite group $\Gamma$ (the uniformizing group) acting effectively on $\widetilde{U}$, and a $\Gamma$-invariant map $\varphi: \widetilde{U} \rightarrow X$ which induces a homeomorphism of $\widetilde{U} / \Gamma$ onto an open set $U \subset X$.

In the following given an orbifold chart $(\widetilde{U}, \Gamma, \varphi)$ the image $\varphi(\widetilde{U})$ will always be denoted by $U$.

2) An (orbifold) embedding $\lambda:(\widetilde{U}, \Gamma, \varphi) \rightarrow\left(\widetilde{U}^{\prime}, \Gamma^{\prime}, \varphi^{\prime}\right)$ between two charts is a smooth embedding such that $\varphi^{\prime} \circ \lambda=\varphi$.

3) An orbifold atlas on $X$ is a family of charts $\mathcal{U}=\left\{\left(\widetilde{U}_{i}, \Gamma_{i}, \varphi_{i}\right)\right\}$ satisfying the following two properties:

i) $X=\bigcup_{i} \varphi_{i}\left(\widetilde{U}_{i}\right)$. 
ii) Let $\left(\widetilde{U}_{i}, \Gamma_{i}, \varphi_{i}\right)$ and $\left(\widetilde{U}_{j}, \Gamma_{j}, \varphi_{j}\right)$ be two orbifold charts. For any point $x \in$ $U_{i} \bigcap U_{j}$ there exist a chart $\left(\widetilde{U}_{k}, \Gamma_{k}, \varphi_{k}\right)$ with $x \in U_{k}$ and two embeddings $\lambda_{k i}:\left(\widetilde{U}_{k}, \Gamma_{k}, \varphi_{k}\right) \rightarrow\left(\widetilde{U}_{i}, \Gamma_{i}, \varphi_{i}\right)$ and $\lambda_{k j}:\left(\widetilde{U}_{k}, \Gamma_{k}, \varphi_{k}\right) \rightarrow\left(\widetilde{U}_{j}, \Gamma_{j}, \varphi_{j}\right)$.

4) An atlas $\mathcal{V}$ is a refinement of an atlas $\mathcal{U}$ if every chart in $\mathcal{V}$ has an embedding in some chart of $\mathcal{U}$.

5) Two atlases $\mathcal{U}$ and $\mathcal{V}$ are equivalent if they admit a common refinement.

6) An orbifold $\mathcal{X}=(X, \mathcal{U})$ consists of a second countable Hausdorff space $X$ with an equivalence class of orbifold atlases represented by $\mathcal{U}$.

Notation. We will sometimes denote an orbifold by $\mathcal{X}$, omitting the underlying topological space $X$ and the orbifold atlas $\mathcal{U}$.

Remark 2.2. One can define complex orbifolds in an analogous way. Namely, by replacing $\mathbb{R}^{n}$ by $\mathbb{C}^{n}$ and smooth maps by holomorphic ones. For simplicity, we will work in the real setting, but all results can be translated to the complex case.

One can prove (see [89]) that given two embeddings $\lambda_{1}, \lambda_{2}:(\tilde{U}, \Gamma, \varphi) \rightarrow\left(\tilde{U}^{\prime}, \Gamma^{\prime}, \varphi^{\prime}\right)$ there exists a unique $\gamma^{\prime} \in \Gamma^{\prime}$ such that $\lambda_{2}=\gamma^{\prime} \circ \lambda_{1}$. Therefore the isotropy group of $p \in \widetilde{U}$ depends only on $x=\varphi(p)$ and will be denoted by $\Gamma_{x}$. A point $x \in X$ whose isotropy group $\Gamma_{x}$ is non-trivial is called an orbifold singular point. The set of orbifold singular point is denoted by $\Sigma^{o r b}(\mathcal{X})$. Points for which $\Gamma_{x}=0$ are called regular and form an open dense subset of $X$.

Remark 2.3. Notice that the set $\Sigma(X)$ of singular points of the space $X$ is contained in the set $\Sigma^{o r b}(\mathcal{X})$, i.e. singular points of $X$ are also orbifold singular points for an orbifold $\mathcal{X}=(X, \mathcal{U})$. The converse is false in general. For instance, the global quotient of $S^{2}$ by a finite cyclic group of rotations $\mathbb{Z}_{n}$ is an orbifold with two orbifold singular points. Nevertheless, the underlying topological space is again $S^{2}$.

Orbifolds arise naturally as quotients of a manifold by a smooth and effective action of a finite group. However, not all orbifolds are of this type. In particular, we will see in Chapter 3 that the orbifolds associated to Sasaki structures are not, in general, global quotients by finite groups. However, they turn out to be quotients of a manifold by a Lie group acting smoothly, effectively and with finite isotropy groups.

We conclude this secton by giving the notion of maps between orbifolds. These can be regarded as a collection of equivariant maps between charts which induce the same map on the underlying topological space.

Definition 2.4. Let $\mathcal{X}=(X, \mathcal{U})$ and $\mathcal{Y}=(Y, \mathcal{V})$ be orbifolds.

1) A map $f: X \rightarrow Y$ is smooth if for every point $x \in X$ there exist charts $(\tilde{U}, \Gamma, \varphi)$ around $x$ and $(\widetilde{V}, \Delta, \psi)$ around $f(x)$ and a smooth lift $\tilde{f}_{\widetilde{U}}: \widetilde{U} \rightarrow \widetilde{V}$ of $f$.

2) The orbifolds $\mathcal{X}$ and $\mathcal{Y}$ are equivalent if there exist two smooth maps $f: X \rightarrow Y$ and $g: Y \rightarrow X$ such that $g f=\operatorname{Id}_{X}$ and $f g=\operatorname{Id}_{Y}$. 


\subsection{Orbibundles}

In this section we give the definition of bundles and classical constructions on bundles in the orbifold setting. In general these will be local data together with compatibility conditions that ensure the definition of a global object on an orbifold. The first example is the following.

Definition 2.5. An orbisheaf (or simply sheaf) $\mathcal{F}$ on an orbifold $\mathcal{X}=(X, \mathcal{U})$ consists of a sheaf $\mathcal{F}_{\widetilde{U}_{i}}$ on each chart $\left(\widetilde{U}_{i}, \Gamma_{i}, \varphi_{i}\right)$ satisfying the following compatibility condition. For each embedding $\lambda_{i j}: \widetilde{U}_{i} \rightarrow \widetilde{U}_{j}$ there exists a functorial isomorphism of sheaves $\mathcal{F}\left(\lambda_{i j}\right): \mathcal{F}_{\widetilde{U}_{i}} \rightarrow \lambda_{i j}^{*} \mathcal{F}_{\widetilde{U}_{j}}$.

Next we define orbibundles. These are central objects in this chapter as well as in the study of Sasaki structures. Once more, orbibundles are defined by bundles over each chart which can be "glued together".

Definition 2.6. An orbibundle $E \rightarrow \mathcal{X}$ over an orbifold $\mathcal{X}=(X, \mathcal{U})$ consists of fiber bundles $E_{\widetilde{U}_{i}}$ over the charts $\left(\widetilde{U}_{i}, \Gamma_{i}, \varphi_{i}\right)$ with fiber a manifold $F$ and structure group $G$ together with homomorphisms $h_{\widetilde{U}_{i}}: \Gamma_{i} \rightarrow G$ such that the following conditions hold:

i) Let $p$ be a point in the fiber over $\widetilde{x}_{i} \in \widetilde{U}_{i}$. Then $p h_{\widetilde{U}_{i}}(\gamma)$ lies in the fiber over $\gamma^{-1} \widetilde{x}_{i}$ for all $\gamma \in \Gamma_{i}$.

ii) Let $\lambda_{i j}: \widetilde{U}_{i} \rightarrow \widetilde{U}_{j}$ be an embedding. Given an element $\gamma \in \Gamma_{i}$ let $\gamma^{\prime} \in \Gamma_{j}$ be the unique element such that $\lambda_{i j} \circ \gamma=\gamma^{\prime} \circ \lambda_{i j}$. Then there is a bundle morphism

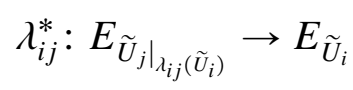

satisfying the conditions that $h_{\widetilde{U}_{i}}(\gamma) \circ \lambda_{i j}^{*}=\lambda_{i j}^{*} \circ h_{\widetilde{U}_{j}}\left(\gamma^{\prime}\right)$. The morphism $\lambda_{i j}^{*}$ is often called a transition map.

iii) Moreover if $\lambda_{j k}: \widetilde{U}_{j} \rightarrow \widetilde{U}_{k}$ is another embedding then $\left(\lambda_{j k} \circ \lambda_{i j}\right)^{*}=\lambda_{i j}^{*} \circ \lambda_{j k}^{*}$.

If the fiber $F$ is a vector space and $G \subset G L(F)$ then $E$ is a vector orbibundle. If $F=G$ and the action is given by right multiplication then $E$ is a principal $G$-orbibundle.

We will sometimes write bundle in place of orbibundle if the meaning is clear from the context.

The total space $E$ of an orbibundle has an orbifold structure induced from the base. Consider orbifold charts $\widetilde{U}_{i}$ on $\mathcal{X}$ which are trivializing sets for $E$, i.e. such that $E_{\widetilde{U}_{i}}$ is the product $\widetilde{U}_{i} \times F$, and define orbifold charts $\left(E_{\widetilde{U}_{i}}, \Gamma_{i}^{*}, \varphi^{*}\right)$ as follows. The action of $\Gamma_{i}$ extends to $E_{\widetilde{U}_{i}}$ as $\left(\widetilde{x}_{i}, p\right) \mapsto\left(\gamma^{-1} \widetilde{x}_{i}, p h_{\widetilde{U}_{i}}(\gamma)\right)$, thus we can define the group $\Gamma_{i}^{*}$ to be the subgroup of $\Gamma_{i}$ that stabilizes the point $\left(x_{i}, p\right)$. The total space $E$ is obtained by gluing together the sets $E_{\widetilde{U}_{i}} / \Gamma_{i}^{*}$. 
Lemma 2.7. Let $P$ be the total space of a principal G-orbibundle over an orbifold $\mathcal{X}=(X, \mathcal{U})$. Then $P$ is a smooth manifold if and only if the maps $h_{\widetilde{U}_{i}}$ are injective for all $i$.

Proof. For a principal orbibundle the group $h_{\widetilde{U}_{i}}\left(\Gamma_{i}^{*}\right) \subset G$ acts freely on the fiber $G$. Therefore if $h_{\widetilde{U}_{i}}$ is injective, there is no element of $\Gamma_{i}$ that stabilizes a point under the action $\left(\tilde{x}_{i}, g\right) \mapsto\left(\gamma^{-1} \tilde{x}_{i}, g h_{\widetilde{U}_{i}}(\gamma)\right)$. Thus the groups $\Gamma_{i}^{*}$ are trivial and so is the orbifold structure on $P$.

Example 2.8 (The tangent bundle of $\mathcal{X})$. Let $\mathcal{X}=(X, \mathcal{U})$ be an orbifold. For each chart $\left(\widetilde{U}_{i}, \Gamma_{i}, \varphi_{i}\right) \in \mathcal{U}$ consider the set $E_{\widetilde{U}_{i}}=T \widetilde{U}_{i}$, i.e. the tangent space of $\widetilde{U}_{i}$. Given an embedding $\lambda_{i j}: \widetilde{U}_{i} \rightarrow \widetilde{U}_{j}$ the differential $D \lambda_{i j}: E_{\widetilde{U}_{i}} \rightarrow E_{\widetilde{U}_{j}}$ satisfies $D \lambda_{i j}(\widetilde{x}) \in G L(n, \mathbb{R})$. Hence we define the transition map for the tangent bundle to be the inverse of $D \lambda_{i j}$. Since every element of $\Gamma_{i}$ defines an embedding the maps $h_{\widetilde{U}_{i}}$ are injective and satisfy the first property in Definition 2.6. Moreover by definition the transition maps satisfy the second condition in Definition 2.6. Therefore we have defined a bundle $T \mathcal{X}=\left(T X, \mathcal{U}^{*}\right)$ where the elements of $\mathcal{U}^{*}$ are given by $\left(\widetilde{U}_{i} \times \mathbb{R}^{n}, \Gamma_{i}^{*}, \varphi_{i}^{*}\right)$ with $\Gamma_{i}^{*}=\Gamma_{i}$ acting linearly on $\mathbb{R}^{n}$ and $\varphi_{i}^{*}$ is the quotient projection. Note that $T X$ is only the notation for the underlying topological space since $X$ is in general not a smooth manifold.

Example 2.9 (The linear frame bundle $L(\mathcal{X})$ of $\mathcal{X}$ ). In the notation of Example 2.8 set now $G=F=G L(n, \mathbb{R})$ and let $E_{\widetilde{U}_{i}}$ be the linear frame bundle over $\widetilde{U}_{i}$. We define the linear frame bundle $L(\mathcal{X})=\left(L(X), \mathcal{U}^{*}\right)$ to have charts of the form $\left(\widetilde{U}_{i} \times\right.$ $\left.G L(n, \mathbb{R}), \Gamma_{i}^{*}, \varphi^{*}\right)$. Also in this case $\varphi_{i}^{*}$ is the quotient projection of the action of $\Gamma_{i}^{*}=\Gamma_{i}$ given by $(\widetilde{x}, A) \mapsto\left(\gamma^{-1} \widetilde{x}, A h_{\widetilde{U}_{i}}(\gamma)\right)$ where $h_{\widetilde{U}_{i}}$ is defined as in Example 2.8. Notice that the homomorphisms $h_{\widetilde{U}_{i}}$ are injective, thus by Lemma 2.7 the total space $L(X)$ is a smooth manifold. However, the action of $G L(n, \mathbb{R})$ on $L(X)$ is only locally free; indeed, the isotropy groups are given by the uniformizing groups $\Gamma_{i}$.

Definition 2.10. Let $\mathcal{E}=\left(E, \mathcal{U}^{*}\right)$ be an orbibundle over $\mathcal{X}$. A section $s$ of $\mathcal{E}$ over $U \subset X$ consists of a section $s_{i}$ of the bundle $E_{\widetilde{U}_{i}}$ for each chart $\left(\widetilde{U}_{i}, \Gamma_{i}, \varphi_{i}\right)$ such that $U_{i} \subset U$ satisfying, for all $\tilde{x} \in \widetilde{U}_{i}$, the following properties.

i) For each $\gamma \in \Gamma_{i}, s_{i}\left(\gamma^{-1} \widetilde{x}\right)=s_{i}(\widetilde{x}) h_{\widetilde{U}_{i}}(\gamma)$.

ii) If $\lambda_{i j}: \widetilde{U}_{i} \rightarrow \widetilde{U}_{j}$ is an embedding, then $\lambda_{i j}^{*} s_{j}\left(\lambda_{i j}(\widetilde{x})\right)=s_{i}(\widetilde{x})$.

We can carry out a construction analogous to Example 2.8 in order to define the cotangent orbibundle and the tensor orbibundles. Combining these with the previous definition allows us to define vector fields, differential forms, metrics, connections, etc. in parallel to the smooth case. For instance, we have the following

Definition 2.11. A Riemannian metric on an orbifold $\mathcal{X}=(X, \mathcal{U})$ is a collection of metrics on each chart $\left(\widetilde{U}_{i}, \Gamma_{i}, \varphi_{i}\right) \in \mathcal{U}$ such that $\Gamma_{i}$ acts by isometries and every embedding $\lambda_{i j}: \widetilde{U}_{i} \rightarrow \widetilde{U}_{j}$ is an isometry. 
Similarly we can define Hermitian metrics on complex orbifolds. Moreover we have the following

Proposition 2.12 ([88]). Every orbifold admits a Riemannian metric and every complex orbifold admits a Hermitian metric.

Proof. The proof is analogous to the smooth case. Namely, one makes use of a partition of unity to patch together metrics on $\widetilde{U}_{i}$ which are invariant under $\Gamma_{i}$.

Notice that differential forms and the exterior derivative are well defined on an orbifold $\mathcal{X}$. Therefore we can define the de Rham complex $\Omega_{d R}(\mathcal{X})$ and, consequently, its cohomology $H_{d R}^{*}(\mathcal{X})$. In his seminal paper Satake already noticed that de Rham's theorem holds in the orbifold setting. In particular we have

Proposition 2.13 ([104]). For any orbifold $\mathcal{X}=(X, \mathcal{U})$ the de Rham cohomology ring $H_{d R}^{*}(X)$ is isomorphic to the singular cohomology ring with real coefficients $H^{*}(X ; \mathbb{R})$.

Example 2.14 (The frame bundle $\operatorname{Fr}(\mathcal{X})$ of $\mathcal{X}$ ). In light of Proposition 2.12 we can define the (orthonormal) frame orbibundle of an orbifold $\mathcal{X}=(X, \mathcal{U})$. The construction is analogous to that of the linear frame bundle. Namely we patch together the orthonormal frame bundles $E_{\widetilde{U}_{i}}$ over each chart $\left(\widetilde{U}_{i}, \Gamma_{i}, \varphi_{i}\right) \in \mathcal{U}$. We obtain in this way a subbundle $\operatorname{Fr}(\mathcal{X})=\left(\operatorname{Fr}(X), \mathcal{U}^{*}\right)$ of the linear frame bundle $L(\mathcal{X})$. Moreover, since the homomorphisms $h_{\widetilde{U}_{i}}$ are injective, the total space is again a smooth manifold.

Proposition 2.15. Every orbifold is the quotient orbifold of a locally free action of a compact Lie group on a smooth manifold.

Proof. The frame bundle $\operatorname{Fr}(\mathcal{X})$ of an orbifold $\mathcal{X}$ is a smooth manifold, see Example 2.14. Moreover, by definition $\mathcal{X}$ is the quotient orbifold of the $O(n)$ action on $\operatorname{Fr}(\mathcal{X})$. The claim follows immediately.

\subsection{Orbifold classifying spaces}

We will now present an equivalent definition of orbifolds that will allow us to define some key topological invariants of orbifolds. We start by describing the correspondence between orbifolds and proper effective étale Lie groupoids. Recall that a category is small if its objects and morphisms form sets.

Definition 2.16. 1) A groupoid (5 is a small category whose morphisms are isomorphisms.

2) A Lie groupoid is a groupoid whose sets of objects $G_{0}$ and morphisms $G_{1}$ are smooth manifolds, the following two maps are smooth submersions

i) the source map $s: G_{1} \rightarrow G_{0} s(g: x \rightarrow y)=x$, 
ii) the target map $t: G_{1} \rightarrow G_{0} t(g: x \rightarrow y)=y$,

and the following three maps are smooth

iii) the composition map $m: G_{1 s} \times{ }_{t} G_{1} \rightarrow G_{1}$ where $G_{1 s} \times{ }_{t} G_{1}=\{(h, g) \in$ $\left.G_{1} \times G_{1} \mid t(g)=s(h)\right\}$,

iv) the identity map $u: G_{0} \rightarrow G_{1}$,

v) the inverse map $i: G_{1} \rightarrow G_{1}$.

3) A Lie groupoid $(5$ is an étale groupoid if $s$ and $t$ are local diffeomorphisms.

4) A Lie groupoid 65 is proper if the map $(s, t): G_{1} \rightarrow G_{0} \times G_{0}$ is proper.

5) An étale Lie groupoid $\left(5\right.$ is effective if the isotropy group $G_{x}=\left\{g \in G_{1} \mid s(g)=\right.$ $t(g)=x\}$ acts effectively on $G_{0}$.

Notice that given a Lie groupoid $\left(55\right.$ we can define the space of orbits $X_{\mathfrak{5}}$ to be the quotient of $G_{0}$ under the following equivalence relation

$$
x \sim y \Longleftrightarrow \exists g \in G_{1} \text { such that } s(g)=x \text { and } t(g)=y .
$$

Example 2.17. An example of Lie groupoid that will be relevant for us is the action groupoid $G \ltimes M$. Given a Lie group $G$ acting on a manifold $M$ let $(G \ltimes M)_{0}=M$ and $(G \ltimes M)_{1}=G \times M$, define the source map to be the projection on the second factor and the target map to be $t(g, x)=g(x)$. The composition map is then given by multiplication in $G$.

Remark 2.18. If $M$ is a quasi-regular Sasaki manifold then we can associate to it two different action groupoids. Namely, the first and more immediate Lie groupoid is given by the $S^{1}$-action on $M$. Now let $\pi: M \rightarrow \mathcal{X}$ be the projection on the space of orbits and consider the $O(2 n)$ action on the frame bundle of $\mathcal{X}$. This is again an action of a Lie group on a smooth manifold therefore it defines another action groupoid.

We will see that one can associate to $\mathcal{X}$ yet another Lie groupoid and the three of them are equivalent in a sense that we will explain below. First, in order to formulate this equivalence, we need some definitions.

Definition 2.19. A homomorphism of Lie groupoids is a functor $\Phi: \mathfrak{5} \rightarrow \mathfrak{H}$ such that the maps $\Phi_{0}: G_{0} \rightarrow H_{0}$ and $\Phi_{1}: G_{1} \rightarrow H_{1}$ are smooth.

Definition 2.20. A homomorphism $\Phi: \mathfrak{b} \rightarrow \mathfrak{H}$ of Lie groupoids is an equivalence if and only if

i) the map $t \circ \mathrm{pr}_{1}: H_{1 s} \times{ }_{\Phi} G_{0} \rightarrow H_{0}$, is a surjective submersion (where $H_{1 s} \times_{\Phi} G_{0}=$ $\left.\left\{(h, x) \in H_{1} \times G_{0} \mid s(h)=\Phi(x)\right\}\right)$, and 
ii) the diagram

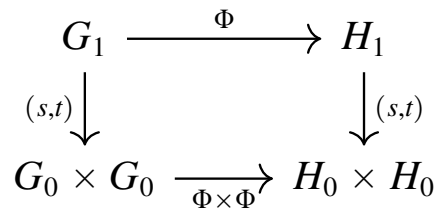

is a pullback diagram.

Definition 2.21. Two Lie groupoids $\mathfrak{G}$ and $\mathfrak{G}^{\prime}$ are said to be Morita equivalent if there exists a third Lie groupoid $\mathfrak{S}$ and two equivalences $\Phi: \mathfrak{H} \rightarrow\left(\mathfrak{5}\right.$ and $\Phi^{\prime}: \mathfrak{H} \rightarrow\left(\mathfrak{\zeta}^{\prime}\right.$.

Now given an orbifold $\mathcal{X}=(X, \mathcal{U})$ we want to construct a proper effective étale Lie groupoid which captures the information of the orbifold structure. Let $U=\sqcup_{i} \widetilde{U}_{i}$ be the disjoint union of all charts $\widetilde{U}_{i} \in \mathcal{U}$. Denote by $\mathcal{P}_{\mathcal{X}}$ the pseudogroup generated by embeddings $\lambda_{i j}$ and their inverses. We can identify $X$ with the quotient $U / \mathcal{P}_{X}$ where two points $x, y$ are identified if there exists an element $f \in \mathcal{P}_{X}$ such that $f(x)=y$. Now consider the groupoid $\mathfrak{5}_{X}$ whose set of objects is $U$ and whose morphisms are germs of the embeddings. It is clear that the orbit space of $\mathfrak{5}_{X}$ is homeomorphic to $X$. Notice that the groupoid $\mathfrak{5}_{X}$ depends on the orbifold atlas $\mathcal{U}$, nonetheless we have the following result.

Theorem $2.22([89])$. Let $\mathcal{X}=(X, \mathcal{U})$ be an orbifold. Then $\mathfrak{b}_{X}$ is a proper effective étale Lie groupoid. Moreover, if $\mathcal{X}^{\prime}=\left(X^{\prime}, \mathcal{U}^{\prime}\right)$ is another orbifold, then $\mathfrak{5}_{X}$ and $\mathfrak{5}_{X^{\prime}}$ are Morita equivalent if and only if $\mathcal{X}$ and $\mathcal{X}^{\prime}$ are equivalent.

Conversely, it is then clear that we can associate an orbifold $X_{\mathfrak{F}}=\left(X_{\mathfrak{G}}, \mathcal{U}_{\mathfrak{F}}\right)$ to a proper effective étale Lie groupoid $\mathfrak{5}$ in a straightforward way. Namely, let $X_{\mathfrak{5}}$ be as above and let $U_{x}$ be a neighbourhood of a point $x \in G_{0}$ which is diffeomorphic to an open set in $\mathbb{R}^{n}$, i.e. there exists a diffeomorphism $\phi_{x}: \widetilde{U}_{x} \rightarrow U_{x}$. Then, with a slight abuse of notation, the charts are given by $\left(\widetilde{U}_{x}, G_{x}, \pi \circ \phi_{x}\right)$ where $\pi: G_{0} \rightarrow X_{\mathfrak{G}}$ is the quotient projection. Thus, given a proper effective étale Lie groupoid $\mathbb{6}_{5}$ the orbit space $\mathcal{X}_{\mathfrak{G}}$ has a canonical orbifold structure. This shows that proper effective étale Lie groupoids correspond exactly to orbifold structures on second countable Hausdorff spaces.

We are now interested in finding a classifying space for orbifolds using the above correspondence with Lie groupoids. In order to do so we need to introduce the concept of principal $\mathfrak{6} \mathfrak{5}$-bundle for a Lie groupoid $\mathfrak{5}$.

Definition 2.23. Let $\mathfrak{5}$ be a Lie groupoid and $Y$ a topological space. Given a continuous map $\mu: Y \rightarrow G_{0}$ let $G_{1 s} \times{ }_{\mu} Y$ be as in Definition 2.20.

1) The action of $\left(5\right.$ on $Y$ with moment map $\mu$ is a map $\mathcal{A}: G_{1 s} \times_{\mu} Y \rightarrow Y$ such that

i) $\mu(\mathcal{A}(g, y))=t(g)$,

ii) $\mathcal{A}(g \circ h, y)=\mathcal{A}(g, \mathcal{A}(h, y))$,

iii) $\mathcal{A}(u(\mu(y)), y)=y$. 
The orbit of $(55$ through $y$ is the subset $[5(y)=\{\mathcal{A}(g, y) \subset Y \mid s(g)=\mu(y)\}$.

2) A principal (5-bundle over $Y$ is a topological space $E$ with a surjection $\pi: E \rightarrow Y$ and an action of $\left(55\right.$ on $E$ with moment map $\mu: E \rightarrow G_{0}$ such that $\pi(\mathcal{A}(g, e))=$ $\pi(e)$ for $e \in E$. Moreover the action is transitive on the fibers and each point $y \in Y$ has an open neighbourhood $U$ and a section $\sigma: U \rightarrow E$ such that the map

$$
\begin{aligned}
G_{1 s} \times{ }_{\mu} U & \longrightarrow \pi^{-1}(U) \\
(g, y) & \mapsto \mathcal{A}(g, \sigma(y))
\end{aligned}
$$

is a homeomorphism.

Theorem 2.24 ([55, 56]). Given a Lie groupoid $(5$ there exists a principal 5 -bundle $\pi: E 6_{5} \rightarrow B(5$ which is universal in the sense that for any $C W$-complex $X$ the pullback $E \mathbb{5} \mapsto f^{*} E(5)$ induces a one-to-one correspondence between homotopy classes of maps $f: Y \rightarrow B\left(5\right.$ and isomorphism classes of principal $\left(\mathfrak{5}\right.$-bundles over $Y$. Moreover if $\mathfrak{5}^{\prime}$ is Morita equivalent to $\mathbf{6}_{5}$ then $B(5)$ and $B\left(\mathfrak{G}^{\prime}\right.$ are weakly homotopy equivalent.

For action groupoids, defined in Example 2.17, the classifying space admits a more concrete description. Namely, let $\mathfrak{5}=G \ltimes M$ be an action groupoid. Then a classifying space $B\left(\mathfrak{5}\right.$ is the space $E G \times{ }_{G} M$, i.e. the quotient of $E G \times M$ by the diagonal action of $G$. See [82] for a proof.

In the case of interest to us, i.e. a proper effective étale Lie groupoid $\mathfrak{5}_{\mathcal{X}}$ arising from an orbifold $\mathcal{X}$, we can describe the universal principal $\mathfrak{5}_{\mathcal{X}}$-bundle more explicitely in terms of $\mathcal{X}$. Recall that any orbifold $\mathcal{X}$ can be seen as the quotient of its frame bundle $\operatorname{Fr}(\mathcal{X})$ by the $O(n)$ action. Let $\operatorname{Fr}(U)$ be the disjoint union of the frame bundles over the charts $\widetilde{U}_{i} \in \mathcal{U}$. It is clear that the groupoid action on $U$ induces a $\mathfrak{G}_{X}$-action on $\operatorname{Fr}(U)$ whose orbit space is $\operatorname{Fr}(\mathcal{X})$. Therefore we can set $E \mathfrak{b}_{\mathcal{X}}=\operatorname{Fr}(U) \times_{O(n)} E O(n)$. Moreover, by requiring $\mathfrak{b}_{X}$ to act trivially on the second factor, we can see that $E \mathfrak{b}_{X}$ is a principal $\mathfrak{5}_{\mathcal{X}}$-bundle over $\operatorname{Fr}(\mathcal{X}) \times_{O(n)} E O(n)$.

By construction we have a commutative diagram

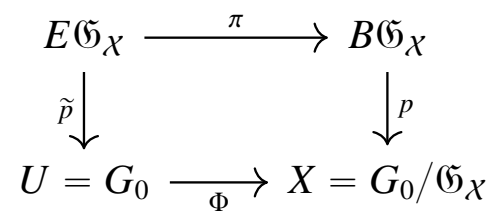

where the horizontal arrows are $\mathfrak{5}_{\mathcal{X}}$-bundles and the vertical arrows are $O(n)$-bundles.

Moreover, by Theorem 2.22 and Theorem 2.24, the homotopy type of $B 5_{X}$ only depends on the equivalence class of $\mathcal{X}$. This gives rise to the following fundamental

Definition 2.25. The orbifold classifying space $B X$ of an orbifold $X$ is the classifying space $B \mathfrak{5}_{X}$ of the associated proper effective étale Lie groupoid $\mathfrak{5}_{X}$. 
Definition 2.26. The orbifold homotopy, homology and cohomology groups of an orbifold $\mathcal{X}$ are defined by

$$
H_{\text {orb }}^{i}(\mathcal{X} ; \mathbb{Z})=H^{i}(B \mathcal{X} ; \mathbb{Z}), H_{i}^{\text {orb }}(\mathcal{X} ; \mathbb{Z})=H_{i}(B \mathcal{X} ; \mathbb{Z}), \pi_{i}^{\text {orb }}(\mathcal{X})=\pi_{i}(B \mathcal{X}) .
$$

Before showing that $B X$ does indeed classify orbibundles on $X$ we need to give a different orbifold atlas on $B X$ that will allow us to define local data and patch them together. Consider an orbifold $\mathcal{X}$ with charts $\left(\widetilde{U}_{i}, \Gamma_{i}, \varphi_{i}\right)$ and let $\operatorname{Fr}(\mathcal{X})$ be its frame bundle with the atlas defined in Example 2.14. Since the orthogonal group $O(n)$ acts locally freely on a chart $E_{\widetilde{U}_{i}}$, we have the homeomorphisms $E_{\widetilde{U}_{i}} / O(n) \simeq \widetilde{U}_{i} / \Gamma_{i} \simeq U_{i}$. Now we define an atlas of $\operatorname{Fr}(\mathcal{X})$ since we can cover it by charts of the form $\widetilde{U}_{i} \times_{\Gamma_{i}}$ $E O(n)$. Moreover, any embedding $\lambda_{i j}: \widetilde{U}_{i} \rightarrow \widetilde{U}_{j}$ induces an embedding $\Lambda_{i j}: \widetilde{U}_{i} \times_{\Gamma_{i}}$ $E O(n) \rightarrow \widetilde{U}_{j} \times_{\Gamma_{j}} E O(n)$ given by $\Lambda_{i j}\left[\left(\widetilde{x}_{i}, p\right)\right]=\left[\left(\lambda_{i j}\left(\widetilde{x}_{i}\right), p\right)\right]$. This allows us to glue together local data on the charts $\widetilde{U}_{i} \times_{\Gamma_{i}} E O(n)$ given by functions on $\widetilde{U}_{i}$ and $E O(n)$ invariant under $\Gamma_{i}$. As an example consider the following diagram

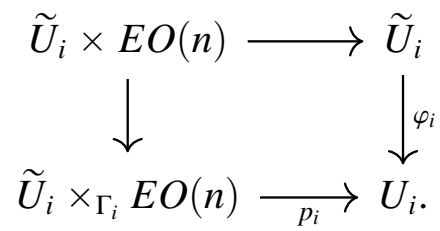

Since the local covering maps are smooth and $\Gamma_{i}$ invariant, by definition the induced map $p: B X \rightarrow X$ is continuous.

Remark 2.27. Notice that one can consider charts of the form $\widetilde{U}_{i} \times_{\Gamma_{i}} E O(n)$ around the fiber of $p: B X \rightarrow X$ over a point $x \in X$. Moreover, for $U_{i}$ small enough and $x$ a regular point the chart is homotopy equivalent to $E O(n)$, and therefore contractible, while when $x$ is a singular point the chart is an Eilenberg-MacLane space $K\left(\Gamma_{i}, 1\right)$. This implies that the cohomology groups $H^{j}\left(p^{-1}\left(U_{i}\right) ; R\right)$ vanish for $R=\mathbb{Q}, \mathbb{R}, \mathbb{C}$. Thus the Leray spectral sequence implies that the map $p: B X \rightarrow X$ induces an isomorphism in cohomology, i.e. $H_{\text {orb }}^{*}(X ; R) \cong H^{*}(X ; R)$.

Remark 2.28. Combining the discussion in Remark 2.27 with Proposition 2.13 yields an isomorphism $H_{o r b}^{*}(\mathcal{X} ; \mathbb{R}) \cong H_{d R}^{*}(\mathcal{X})$. That is, every closed form on $\mathcal{X}$ represents a cohomology class with real coefficients in $H_{o r b}^{*}(\mathcal{X} ; \mathbb{R})$.

Next we explain in which sense the space $B X$ is classifying. Namely, we give a correspondence between orbibundles on $X$ with structure group $G$ and generic fiber $F$ and isomorphism classes of bundles on $B X$ with structure group $G$ and fiber $F$.

Given such an orbibundle on $\mathcal{X}$ we can define an action of $\Gamma_{i}$ on $\widetilde{U}_{i} \times F \times E O(n)$ by $\left(\widetilde{x}_{i}, f, p\right) \mapsto\left(\gamma^{-1} \widetilde{x}_{i}, f h_{\widetilde{U}_{i}}(\gamma), p \gamma\right)$. This defines a $G$-bundle with fiber $F$ on $\widetilde{U}_{i} \times_{\Gamma_{i}} E O(n)$ for all charts $\left(\widetilde{U}_{i}, \Gamma_{i}, \varphi_{i}\right)$. Moreover the second property in Definition 2.6 ensures that the cocycle conditions are satisfied to give a globally defined bundle on $B X$ with structure group $G$ and fiber $F$. 
Conversely, given such a bundle on $B X$, we can restrict to a chart $\widetilde{U}_{i} \times_{\Gamma_{i}} E O(n)$ to get a $G$-bundle with fiber $F$ there. Now, since the chart $\widetilde{U}_{i} \times_{\Gamma_{i}} E O(n)$ is an EilenbergMacLane space $K\left(\Gamma_{i}, 1\right)$, there is a one-to-one correspondence between isomorphism classes of $G$-bundles on $\widetilde{U}_{i} \times_{\Gamma_{i}} E O(n)$ and conjugacy classes of homomorphisms from $\Gamma_{i}$ to $G$. This the defines the homomorphisms $h_{\widetilde{U}_{i}}$ in Definition 2.6.

Proposition 2.29. The correspondence above between isomorphism classes of orbibundles on $\mathcal{X}$ with structure group $G$ and generic fiber $F$ and isomorphism classes of bundles on $B X$ with structure group $G$ and fiber $F$ is one-to-one.

Since $B \mathcal{X}$ is a $\mathrm{CW}$-complex, the following result is an immediate consequence.

Corollary 2.30. For any Lie group $G$ there is a one-to-one correspondence between principal $G$-orbibundle on an orbifold $\mathcal{X}$ and homotopy classes of maps $[B \mathcal{X}, B G]$.

Moreover, we get the following corollary whose proof is a straightforward adaptation of the proof in the smooth case.

Corollary 2.31. Isomorphism classes of G-bundles on $\mathcal{X}$ are in one-to-one correspondence with the cohomology set $H_{\text {orb }}^{1}(X, \mathfrak{5})=H^{1}(B X, \mathfrak{5})$ with 5 denoting the sheaf of germs of functions to $G$.

Characteristic classes of a smooth bundle can be defined as the pullback of the generators of the cohomology of the structure group. The results above allow us to define orbifold characteristic classes analogously. Namely, since a principal $G$-bundle on $\mathcal{X}$ is uniquely determined by the homotopy class of a map $f: B X \rightarrow B G$, we define the orbifold characteristic classes to be the elements in the image of $f^{*}: H^{*}(B G, R) \rightarrow$ $H_{o r b}^{*}(X, R)$ for a ring $R$. For instance to any orbibundle with structure group $O(n), U(n)$ or $S p(n)$ we can associate Stiefel-Whitney, Chern or Pontryagin classes respectively. Since our focus will be on complex vector bundles we give the following

Definition 2.32. Let $E \rightarrow \mathcal{X}$ be an orbibundle with structure group $U(n)$ and let $f$ : $B X \rightarrow B U(n)$ be its classifying map. The $i$-th orbifold Chern class $c_{i}^{\text {orb }}(E)$ of $E$ are defined to be

$$
f^{*}\left(c_{i}\right) \in H^{2 i}(B \mathcal{X} ; \mathbb{Z})
$$

where the $c_{i} \in H^{2 i}(B U(n) ; \mathbb{Z})$ are such that $H^{*}(B U(n) ; \mathbb{Z}) \cong \mathbb{Z}\left[c_{1}, c_{2}, \ldots, c_{n}\right]$.

On a smooth manifold $M$ Chern-Weil theory gives an equivalent definition of characteristic classes. In particular it provides differential forms representing the image of Chern classes under the inclusion $H^{*}(M ; \mathbb{Z}) \hookrightarrow H_{d R}^{*}(M)$. Since all the objects involved are well defined on orbifolds the theory translates in the orbifold setting, see [31] for the details. Namely, for a $U(n)$-orbibundle $E$ consider the image $c_{i}^{\text {orb }}(E)_{\mathbb{R}}$ of the orbifold Chern classes $c_{i}^{\text {orb }}(E)$ under the inclusion $H_{o}^{*} r b(\mathcal{X} ; \mathbb{Z}) \hookrightarrow H_{o}^{*} r b(\mathcal{X} ; \mathbb{R})$. To each connection 1-form on $E$ the Chern-Weil homomorphism associates differential forms on $\mathcal{X}$. These forms represent the classes $c_{i}^{\text {orb }}(E)_{\mathbb{R}}$ under the isomorphism given in Remark 2.28, 
Definition 2.33. Let $E$ be a $U(n)$-orbibundle. The $i$-th real orbifold Chern class is the class $c_{i}^{\text {orb }}(E)_{\mathbb{R}} \in H_{\text {orb }}^{2 i}(\mathcal{X} ; \mathbb{R}) \cong H_{d R}^{2 i}(\mathcal{X})$ defined above.

Notation. When it will be clear from the context we will write simply $c_{i}^{\text {orb }}(E)$ for both real and integral orbifold Chern classes. Moreover we will sometimes write $C_{i}(E)$ dropping the superscript in order to lighten the notation.

We can now prove the orbifold analogue of a classical result.

Proposition 2.34. Principal $S^{1}$-bundles $E$ over an orbifold $\mathcal{X}$ are are in one-to-one correspondence with elements of $H_{o r b}^{2}(X, \mathbb{Z})$ and the correspondence is given by the first orbifold Chern class $c_{1}^{\text {orb }}(E)$.

Proof. Let $\mathcal{E}$ be the sheaf of germs of smooth functions $B \mathcal{X} \rightarrow \mathbb{C}$ and $\mathcal{E}^{*}$ the subsheaf of non-vanishing functions. The complex line bundles on $B X$ (up to isomorphism) are in one-to-one correspondence with classes in $H_{o r b}^{1}\left(\mathcal{X}, \mathcal{E}^{*}\right)$. Since $B \mathcal{X}$ is a second countable $\mathrm{CW}$-complex and $\mathcal{E}$ is a fine sheaf the exponential sequence yields an isomorphism $H_{\text {orb }}^{1}\left(\mathcal{X}, \mathcal{E}^{*}\right) \cong H_{\text {orb }}^{2}(\mathcal{X}, \mathbb{Z})$. Now denote by $\mathcal{S}$ the subsheaf of $\mathcal{E}$ given by germs of functions from $B \mathcal{X}$ to $S^{1}$. Since $\mathcal{E}^{*}$ deformation retracts onto $\mathcal{S}$ we get an isomorphism $H_{\text {orb }}^{1}\left(\mathcal{X}, \mathcal{E}^{*}\right) \cong H_{\text {orb }}^{1}(\mathcal{X}, \mathcal{S})$. The statement then follows from the fact that for $\mathrm{CW}$-complexes the connecting morphism in the long exact sequence associated to the exponential sequence is given by the first Chern class.

The last result we include in this chapter is pivotal and we will rely on it several times in the reminder of the thesis. Before we can state it we give the following definition for clarity albeit it is a special case of Definition 2.23 when regarding a Lie group $G$ as a groupoid with set of morphism given by $G$ and a singleton as set of objects.

Definition 2.35. The action of a Lie group $G$ on an orbifold $X=(X, \mathcal{U})$ is given by a continuous action $\mathcal{A}: G \times X \rightarrow X$ such that for each $g \in G$ and $x \in X$ there are charts $\left(\widetilde{U}_{i}, \Gamma_{i}, \varphi_{i}\right)$ and $\left(\widetilde{U}_{j}, \Gamma_{j}, \varphi_{j}\right)$ over $x$ and $\mathcal{A}(g, x)$ respectively with a neighbourhood $V \subset G$ of $g$ and a smooth map $\widetilde{\mathcal{A}}: V \times \widetilde{U}_{i} \rightarrow \widetilde{U}_{j}$ such that $\varphi_{j}(\widetilde{\mathcal{A}}(g, \widetilde{x}))=\mathcal{A}\left(g, \varphi_{i}(\widetilde{x})\right)$ and for all $g \in G$ the map defined by $\widetilde{x} \mapsto \tilde{\mathcal{A}}(g, \tilde{x})$ is a diffeomorphism.

Now to a locally free action of a Lie group $G$ on an orbifold $\mathcal{Y}$ with quotient orbifold $\mathcal{X}$ we associate the fibration $G \times E O(n) \hookrightarrow B Y \rightarrow B X$. This gives rise to a long exact sequence of homotopy groups. We state this important result in the setting that interest us the most.

Theorem 2.36 ([57]). Let $G$ be a torus acting locally freely on an orbifold $y$ with quotient orbifold $\mathcal{X}$. Then the following sequence of homotopy groups

$$
\cdots \rightarrow \pi_{i}(G) \rightarrow \pi_{i}^{o r b}(\mathcal{Y}) \rightarrow \pi_{i}^{o r b}(\mathcal{X}) \rightarrow \pi_{i-1}(G) \rightarrow \cdots
$$

is exact. 


\subsection{Complex cyclic orbifolds}

In this final section we focus on the class of orbifolds that will appear in the rest of the thesis. Namely, complex projective cyclic orbifolds. Moreover, we discuss Seifert bundles and their relation to $S^{1}$-orbibundles with particular emphasis on the smoothness of the total space. For further details on the material presented in this section see [15, Chapter 4] as well as [73, 74].

Recall that for a complex orbifold $\mathcal{X}=(X, \mathcal{U})$ the trivialization charts $\widetilde{U}_{x}$ are biholomorphic to the polydisc $D^{n} \subset \mathbb{C}^{n}$ and the uniformizing groups $\Gamma_{x}$ are finite subgroups of $G L(n, \mathbb{C})$. Moreover, $\mathcal{X}$ is cyclic if the groups $\Gamma_{x}$ are cyclic for all $x \in X$.

In the following we will consider orbifolds whose underlying space $X$ is a normal projective variety. In this case we consider Weil divisors $D_{\alpha}$ which lie in the orbifold singular set $\Sigma^{o r b}(\mathcal{X})$. This in particular implies that the local uniformizing group $\Gamma_{x}$ is non-trivial for all points $x \in D_{\alpha}$. Thus we define the ramification index $m_{\alpha}$ of the divisor $D_{\alpha}$ to be the gcd of all the orders of the uniformizing groups $\Gamma_{x}$ for $x \in D_{\alpha}$. The branch divisor $\Delta$ of the orbifold $\mathcal{X}=(X, \mathcal{U})$ is defined to be the the $\mathbb{Q}$-divisor, i.e., a Weil divisor with coefficients in $\mathbb{Q}$, of the form

$$
\Delta=\sum_{\alpha}\left(1-\frac{1}{m_{\alpha}}\right) D_{\alpha}
$$

where the sum is taken over all divisors that lie in the orbifold singular set $\Sigma^{\text {orb }}(\mathcal{X})$ and the $m_{\alpha}$ 's are as above. Therefore, we can associate a pair $(X, \Delta)$ to a complex cyclic orbifold $(X, \mathcal{U})$ such that $X$ is a normal projective variety. Conversely, the pair $(X, \Delta)$ determines the orbifold $X$ uniquely. In fact, the chart $\varphi_{i}: \widetilde{U}_{i} \cong D^{n} \longrightarrow U_{i}$ at a point $x \in X$, and therefore the atlas $\mathcal{U}$, is uniquely determined by the following conditions:

- $\varphi_{i}$ is unramified over $U_{i} \backslash\left(\Sigma\left(U_{i}\right) \bigcup D_{\alpha}\right)$ where the union is taken over all divisors $D_{\alpha}$ intersecting $U_{i}$ and

- the ramification index $m_{\alpha}$ is the largest integer that divides the ramification index of all $\varphi_{i}: \widetilde{U}_{i} \longrightarrow U_{i}$ such that the intersection $U_{i} \bigcap D_{\alpha}$ is non-empty.

Thus we will often think of complex cyclic orbifolds as pairs $(X, \Delta)$.

Example 2.37 (Weighted projective spaces). Consider the weighted $\mathbb{C}^{*}(w)$-action on $\mathbb{C}^{n+1}$ with weight $w=\left(w_{0}, \ldots, w_{n}\right)$ defined by

$$
\left(z_{0}, \ldots, z_{n}\right) \mapsto\left(\lambda^{w_{0}} z_{0}, \ldots, \lambda^{w_{n}} z_{n}\right) .
$$

We will assume that $w$ satisfies $w_{0} \leqslant w_{1} \leqslant \ldots \leqslant w_{n}$ and $\operatorname{gcd}\left(w_{0}, \ldots, w_{n}\right)=1$. This can be always arranged by reordering the coordinates on $\mathbb{C}^{n+1}$ and redefining the coordinate on $\mathbb{C}^{*}$. The weighted projective space $\mathbb{C P}^{n}(w)$ is the quotient $\mathbb{C}^{n+1} / \mathbb{C}^{*}(w)$ and is endowed with the following orbifold structure. The charts $\varphi_{i}: \widetilde{U}_{i} \longrightarrow U_{i}$ are given by a weighted adaptation of the standard charts of $\mathbb{C P}^{n}$. Namely, let $U_{i}=\left\{\left[z_{0}, \ldots, z_{n}\right] \in\right.$ 
$\left.\mathbb{C P}^{n}(w) \mid z_{i} \neq 0\right\}$ and set coordinates $\left(y_{0}, \ldots, y_{i-1}, y_{i+1}, \ldots, y_{n}\right)$ on the open set $\widetilde{U}_{i} \cong \mathbb{C}^{n}$ such that

$$
y_{j}^{w_{i}}=\frac{z_{j}^{w_{i}}}{z_{i}^{w_{j}}} .
$$

The map $\varphi_{i}$ is then given by

$$
\varphi_{i}\left(y_{0}, \ldots, y_{i-1}, y_{i+1}, \ldots, y_{n}\right)=\left(y_{0}^{w_{i}}, \ldots, y_{i-1}^{w_{i}}, y_{i+1}^{w_{i}}, \ldots, y_{n}^{w_{i}}\right) .
$$

This defines an orbifold chart $\left(\widetilde{U}_{i}, \Gamma_{i}, \varphi_{i}\right)$ where $\Gamma_{i} \cong \mathbb{Z}_{w_{i}}$ is the group of $w_{i}$-th rooths of unity in $\mathbb{C}^{*}(w)$. The atlas $\mathcal{U}$ on $\mathbb{C P}^{n}(w)$ consists of the charts $\left(\widetilde{U}_{i}, \mathbb{Z}_{w_{i}}, \varphi_{i}\right)$ together with the intersection $\left(U_{i_{1} \ldots i_{j}}, \mathbb{Z}_{\operatorname{gcd}\left(w_{i_{1}}, \ldots, w_{i_{j}}\right)}, \varphi_{i_{1} \ldots i_{j}}\right)$ where $U_{i_{1} \ldots i_{j}}=\widetilde{U}_{i_{1}} \bigcap \cdots \bigcap \widetilde{U}_{i_{j}}$.

We focus now on the algebraic variety underlying weighted projective spaces. We have the following result of Dolgachev.

Lemma 2.38 ([36]). As algebraic varieties $\mathbb{C} P^{n}(w) \cong \mathbb{C} P^{n} / G_{w}$ where $G_{w}=\mathbb{Z}_{w_{0}} \times$ $\cdots \times \mathbb{Z}_{w_{n}}$. Moreover, $\mathbb{C} P^{n}(w)$ and $\mathbb{C} P^{n} / G_{w}$ are isomorphic as orbifolds if and only if $w=(1, \ldots, 1)$.

In order to study the underlying algebraic variety of weighted projective spaces $\mathrm{CP}^{n}(w)$ let us introduce some notation. For all $i=0, \ldots, n$ we define the following integers:

$$
\begin{aligned}
d_{i} & =\operatorname{gcd}\left(w_{0}, \ldots, w_{i-1}, w_{i+1}, \ldots, w_{n}\right), \\
e_{i} & =\prod_{j \neq i} d_{j}, \\
\bar{w} & =\left(\frac{w_{0}}{e_{0}}, \ldots, \frac{w_{n}}{e_{n}}\right) .
\end{aligned}
$$

It follows directly from these definitions and the assumption $\operatorname{gcd}\left(w_{0}, \ldots, w_{n}\right)=1$ that:

i) the $d_{i}$ 's are pairwise relatively prime,

ii) $d_{i}$ divides $w_{j}$ for all $i \neq j$,

iii) the $e_{i}$ divides $w_{i}$ for all $i$ so that the entries of the vector $\bar{w}$ are integers and $w=\bar{w}$ if and only if $w=(1, \ldots, 1)$.

The following result of Delorme [34] says that, when interested in $\mathbb{C P}^{n}(w)$ as an algebraic variety, we can consider $\mathbb{C P}^{n}(\bar{w})$ instead, see [36] for a proof.

Proposition 2.39 ([34]). There is an isomorphism $\mathbb{C} P^{n}(w) \cong \mathbb{C} P^{n}(\bar{w})$ of algebraic varieties.

Example 2.40. Notice that if $d_{i} \neq 0$, then the weight vector

$$
w^{\prime}=\left(\frac{w_{0}}{d_{i}}, \ldots, w_{i}, \ldots, \frac{w_{n}}{d_{i}}\right)
$$


satisfies the property $\bar{w}=\bar{w}^{\prime}$. Therefore we have an isomorphism of weighted projective spaces $\mathbb{C P}^{n}(w) \cong \mathbb{C P}^{n}\left(w^{\prime}\right)$ as algebraic varieties. For instance, the algebraic variety $\mathbb{C P}^{2}(6,2(6 k-1), 3(6 k-1))$ is isomorphic to the standard projective space $\mathbb{C P}^{2}$. This can be easily verified by applying the argument above first with $d_{0}=6 k-1$, then with $d_{1}=3$ and finally with $d_{2}=2$.

Remark 2.41. Notice that singular set of the orbifold $\mathbb{C P}^{n}\left(w^{\prime}\right)$ consists of the points $\left[0: \ldots: z_{i}: \ldots: 0\right]$ for all $i$ such that $w_{i} \neq 0$. Therefore, in the notation introduced above, the orbifold $\mathbb{C P}^{n}\left(w^{\prime}\right)$ is denoted by $\left(\mathbb{C P}^{n}\left(w^{\prime}\right), \Delta=0\right)$. On the other hand, given a vector $w$ if $d_{i} \neq 0$ for some $i$, then the divisor $D_{i}=\left(z_{i}=0\right)$ is contained in $\Sigma^{o r b}\left(\mathbb{C P}^{n}(w)\right)$ and has ramification index $d_{i}$. Hence $\Delta \neq 0$.

Example 2.42. Let us now clarify with an example the relation between the orbifold $X=\mathbb{C P}^{n}(w)$ and the pair $(X, \Delta)$.

Consider the weighted projective space $\mathbb{C P}^{2}(3,4,6)$. From the description of the orbifold atlas it is clear that the singular locus is given by

$$
\Sigma^{o r b}\left(\mathbb{C P}^{2}(3,4,6)\right)=\left\{z_{0}=0\right\} \bigcup\left\{z_{1}=0\right\} .
$$

Moreover, the divisors $D_{0}=\left(z_{0}=0\right)$ and $D_{1}=\left(z_{1}=0\right)$ have ramification index 2 and 3 respectively. Thus we have $\Delta=\frac{1}{2} D_{0}+\frac{2}{3} D_{1}$. By Proposition 2.39 the orbifold $\mathbb{C P}^{2}(3,4,6)$ is given by $\left(\mathbb{C P}^{2}(1,2,1), \frac{1}{2} D_{0}+\frac{2}{3} D_{1}\right)$. Notice that $\mathbb{C P}^{2}(1,2,1)$ and $\mathrm{CP}^{2}(3,4,6)$ are isomorphic as algebraic varieties, even though they are not isomorphic as orbifolds since the former has trivial branch divisor $\Delta$.

A large class of examples of complex cyclic orbifolds is given by hypersurfaces, or more generally complete intersections, in a weighted projective space $\mathbb{C P}^{n}(w)$.

Definition 2.43. A polynomial $f\left(z_{0}, \ldots, z_{n}\right)$ is called a weighted homogeneous polynomial of degree $d$ and weight $w=\left(w_{0}, \ldots, w_{n}\right)$ if

$$
f\left(\lambda^{w_{0}} z_{0}, \ldots, \lambda^{w_{n}} z_{n}\right)=\lambda^{d} f\left(z_{0}, \ldots, z_{n}\right)
$$

with $\lambda \in \mathbb{C}^{*}$.

If $V$ is a variety of $\mathbb{C}^{n+1}$ defined by weighted homogeneous polynomials $f_{1}, \ldots, f_{r}$ with weights $w=\left(w_{0}, \ldots, w_{n}\right)$, then it is invariant under the $\mathbb{C}^{*}(w)$-action on $\mathbb{C}^{n+1}$. This leads to the following:

Definition 2.44. A weighted variety in $\mathbb{C P}^{n}(w)$ is the zero set of a collection $f_{1}, \ldots, f_{r}$ of weighted homogeneous polynomials with weights $w$. The variety $X$ is called a weighted complete intersection if $r=\operatorname{codim}(X)$. A weighted hypersurface $X$ is the zero locus of a single weighted homogeneous polynomial.

It is natural to require that the weighted variety $X$ carries an orbifold structure which is naturally induced by $\mathbb{C} \mathrm{P}^{n}(w)$. The next proposition gives a necessary condition. 
Proposition 2.45. Let pr: $\mathbb{C}^{n+1} \backslash\{0\} \longrightarrow \mathbb{C} P^{n}(w)$ be the quotient by the $\mathbb{C}^{*}(w)$-action and $\iota: X \longrightarrow \mathbb{C} P^{n}(w)$ be a weighted variety. If $\mathrm{pr}^{-1}(X)$ is smooth, then $\mathbb{C} P^{n}(w)$ naturally induces a locally cyclic orbifold structure $\mathcal{X}=(X, \mathcal{U})$ on $X$.

Proof. Since $\operatorname{pr}^{-1}(X)$ is smooth and invariant under the $\mathbb{C}^{*}(w)$-action we can use charts of $\operatorname{pr}^{-1}(X)$ at a point $y \in \operatorname{pr}^{-1}(x)$ to define orbifold charts at $x \in X$. The orbifold structure on $X$ is then naturally given by the $\mathbb{C}^{*}(w)$-action on $\operatorname{pr}^{-1}(X)$. Moreover, the local uniformizing group $\Gamma_{x}$ at a point $x \in X$ is the uniformizing group at the point $\left.\iota(x) \in \mathbb{C P}^{n} w\right)$. Hence the orbifold $\mathcal{X}$ is locally cyclic.

Example 2.46 (Brieskorn-Pham Polynomials). A Brieskorn-Pham polynomial with exponent $a=\left(a_{0}, \ldots, a_{n}\right)$ is a degree $d$ weighted homogeneous polynomial $f$ of the form

$$
f\left(z_{0}, \ldots, z_{n}\right)=z_{0}^{a_{0}}+\cdots+z_{n}^{a_{n}}
$$

with $a_{i}>1$ for all $i$. Notice that we have $w_{i} a_{i}=d$ for all $i$ and $d=\operatorname{lcm}\left(a_{0}, \ldots, a_{n}\right)$ since we are assuming $\operatorname{gcd}\left(w_{0}, \ldots, w_{n}\right)=1$. The polynomial $f$ defines a weighted hypersurface $X_{f} \subset \mathbb{C P}^{n}(w)$. It is easy to see that the set $\mathrm{pr}^{-1}\left(X_{f}\right)$ is smooth. Thus $X_{f}$ is naturally endowed with a cyclic orbifold structure. Moreover, we see that the branch divisor $\Delta$ that identifies this orbifold structure is

$$
\Delta=\sum_{i}\left(1-\frac{1}{d_{i}}\right) D_{i}
$$

where the $d_{i}$ 's were defined in (2.2) and $D_{i}$ is the hyperplane divisor $\left(z_{i}=0\right) \bigcap X_{f}$.

Before we move on to the definition of a Seifert bundle we recall that a Stein space is a holomorphically convex space such that any compact analytic subset is finite.

Definition 2.47. Let $X$ be a normal complex space. A Seifert bundle over $X$ is a map $\pi: Y \longrightarrow X$ from a normal complex space $Y$ together with a $C^{*}$-action on $Y$ satisfying the following conditions:

- $\pi$ is $\mathbb{C}^{*}$ invariant with the respect to the trivial action on $X$,

- the preimage $\pi^{-1}$ of any open Stein set is Stein and

- For every $x \in X$, the $\mathbb{C}^{*}$-action on the fiber $Y_{x}=\pi^{-1}(x)$ is $\mathbb{C}^{*}$-equivariantly biholomorphic to the standard $\mathbb{C}^{*}$-action on $\mathbb{C}^{*} / \mu_{m}$ for some $m=m(x, Y / X)$, where $\mu_{m} \subset \mathbb{C}^{*}$ denotes the group of $m$-th roots of unity.

One can always assume that $m(x, Y / X)=1$ on a dense open set, that is, one can assume the $\mathbb{C}^{*}$-action to be effective.

Consider now the set of points $\{x \in X \mid m(x, X / Y)>1\}$. This is a closed analytic subset of $X$. It can be written as the union of Weil divisors $D_{i}$ and of a subset of codimension 
at least 2 contained in singular locus $\Sigma(X)$. The multiplicity $m(x, Y / X)$ is constant on a dense open subset of each $D_{i}$. This common value is denoted by $m_{i}$. The $\mathbb{Q}$-divisor

$$
\Delta=\sum_{i}\left(1-\frac{1}{m_{i}}\right) D_{i}
$$

is called the branch divisor of $\pi: Y \longrightarrow X$. When we want to emphasize the branch divisor we will write the Seifert bundle as $\pi: Y \longrightarrow(X, \Delta)$.

It is clear from the description above that if $Y$ is smooth, the pair $(X, \Delta)$ defines a cyclic orbifold as discussed in the beginning of this section. In fact, for every $x \in X$ and any $y \in \pi^{-1}(x)$ let $V_{x}$ be a $\mu_{m}$-invariant smooth hypersurface transverse to $\pi^{-1}(x)$, where $m=m(x, Y / X)$. Then the maps $\varphi_{x}: V_{x}=\widetilde{U}_{x} \longrightarrow V_{x} / \mu_{m}=U_{x}$ give an orbifold structure $\mathcal{X}=(X, \mathcal{U})$. Moreover, the orbifold branch divisor coincides with the branch divisor of $\pi: Y \longrightarrow X$ so that $X=(X, \Delta)$. It follows from the definition of the orbifold $X$ that $\pi: Y \longrightarrow(X, \Delta)$ is a principal $C^{*}$-orbibundle whose local uniformizing groups inject into $C^{*}$, cf. Lemma 2.7

Consider the splitting $\mathbb{C}^{*}=\mathbb{R} \times S^{1}$ of $\mathbb{C}^{*}$ as a Lie group. Since the definition of a Seifert bundle only involve subgroups of $S^{1}$ we can write $Y$ as $M \times \mathbb{R}$ and restrict the Seifert bundle to $M$. By abuse of notation we will call this a Seifert bundle. Clearly, $M$ is smooth if and only if $Y$ is smooth. In this instance the manifold $M$ is a principal $S^{1}$-orbibundle over the orbifold $(X, \Delta)$. We have proven the following:

Theorem 2.48. Every Seifert bundle $\pi: M \rightarrow(X, \Delta)$ with $M$ smooth is a principal $S^{1}$ orbibundle over a cyclic orbifold $\mathcal{X}=(X, \Delta)$ Conversely, every principal $S^{1}$-orbibundle $\pi: M \rightarrow(X, \Delta)$ over a cyclic orbifold $\mathcal{X}=(X, \Delta)$ whose local uniformizing groups inject into $S^{1}$ is a Seifert bundle with $M$ smooth.

The following result of Kollár gives a useful correspondence.

Theorem 2.49 ([73] $)$. Let $X$ be a normal complex space with at worst quotient singularities and $\Delta=\sum\left(1-\frac{1}{m_{i}}\right) D_{i} a \mathbb{Q}$-divisor. There is a one-to-one correspondence between Seifert bundles $\pi: Y \longrightarrow(X, \Delta)$ and the following data:

1. For each $D_{i}$ an integer $0 \leqslant b_{i} \leqslant m_{i}$, relatively prime to $m_{i}$, and

2. a linear equivalence class of Weil divisors [B], i.e. an element of the divisor class group $C l(X)$.

Definition 2.50. Let $\pi: Y \longrightarrow(X, \Delta)$ be a Seifert bundle and let $[B], m_{i}$ and $b_{i}$ as in Theorem 2.49. The first Chern class $c_{1}(Y / X)$ of the Seifert bundle $\pi: Y \longrightarrow(X, \Delta)$ is defined to be the rational homology class

$$
c_{1}(Y / X)=[B]+\sum_{i} \frac{b_{i}}{m_{i}}\left[D_{i}\right] \in H^{2}(X ; \mathbb{Q})
$$


where we identify the linear equivalence class of a divisor with the first Chern class of the associated line orbibundle.

We want to understand the smoothness condition on $M$ in terms of the data that determines the Seifert bundle according to Theorem 2.49. In order to do so let us recall some notions.

Definition 2.51. An element of $G L(n, \mathbb{C})$ is a reflection if it has eigenvalue 1 with multiplicity $n-1$, that is, if it fixes an hyperplane in $\mathbb{C}^{n}$. A finite subgroup $\Gamma \subset G L(n, \mathbb{C})$ generated by reflections is called a reflection group. A finite group $\Lambda \subset G L(n, \mathbb{C})$ is small if it contains no reflection.

Theorem $2.52([\overline{98}])$. Let $\Gamma \subset G L(n, \mathbb{C})$ be a finite grop. Then

1. the quotient $\mathbb{C}^{n} / \Gamma$ is smooth if and only if $\Gamma$ is a reflection group and

2. there exist a small group $\Lambda$ such that $\mathbb{C}^{n} / \Gamma$ and $\mathbb{C}^{n} / \Lambda$ are biholomorphic.

We can now study the local geometry of a Seifert bundle in terms of the $\mathbb{C}^{*}$-action. Let $\pi: Y \longrightarrow(X, \Delta)$ be a Seifert bundle and suppose $X$ has complex dimension $n$. Pick a point $x \in X$ such that $Y$ is smooth along $\pi^{-1}(x)$. Let $(\tilde{U}, \Gamma, \varphi)$ be an orbifold chart at $x$. We can assume that $\widetilde{U}$ is biholomorphic to the polydisc $D^{n}$. Thus we have $U=$ $\varphi(\widetilde{U})=D^{n} / \mu_{m}$ where $m=m(x, Y / X)$. Now picking a generator $\lambda$ of the uniformizing group $\mu_{m}$ and diagonalising the action we get the vector $\left(a_{1}, \ldots, a_{n}\right)$ such that the action on the $i$-th coordinate is given by $z_{i} \mapsto \lambda^{a_{i}} z_{i}$. We can assume that the action is effective, i.e. that $\operatorname{gcd}\left(a_{1}, \ldots, a_{n}, m\right)=1$. Now consider the following integers:

- $m_{i}=\operatorname{gcd}\left(a_{1}, \ldots, a_{i-1}, a_{i+1}, \ldots, a_{n}, m\right)$ and

- $M=\prod m_{i}$.

Notice that the integers $m_{i}$ are relatively prime since $\operatorname{gcd}\left(a_{1}, \ldots, a_{n}, m\right)=1$. Moreover, by construction they are the multiplicities of the irreducible components $D_{i}$ of $\Delta$ passing through $x$. Hence the number of $m_{i} \neq 1$ i the number of irreducible components $D_{i}$ of $\Delta$ passing through $x$.

Now we can give a clear local description of $\mathcal{X}$ at $x$. Namely, since the subgroups $\mu_{m_{i}} \subset \mu_{m}$ fix all but one coordinate hyperplane in $\mathcal{D}^{n}$, they are reflection groups. By part (1) of Theorem 2.52 the quotient $D^{n} / \mu_{M}$ is smooth and biholomorphic to $D^{n}$. Therefore we have that $\mu_{r}$, where $r=m / M$, is a small group. By part (2) of Theorem 2.52 there is a biholomorphism $U \cong D^{n} / \mu_{r}$.

Therefore, at a point $x \in X$ with $\left|\Gamma_{x}\right|=m$ such that $\pi^{-1}(x)$ is smooth we have a factorization $m=m_{1} \cdots m_{n} r$ satisfying the following conditions:

1. The numbers $m_{i}$ are relatively prime and are the multiplicities of the irreducible components $D_{i}$ of $\Delta$ passing through $x$ with the necessary number of 1's if there are less than $n$ components. 
2. As an algebraic variety $U \cong D^{n} / \mu_{r}$.

As a consequence the local divisor class group $C l(X, x)$ at $x$ is $\mu_{r} \cong \mathbb{Z}_{r}$, see [73, Part 24]. Given a Weil divisor passing through $x$ we can consider its restriction to $U \cong D^{n} / \mu_{r}$. This gives a well defined map $R_{x}: C l(X) \longrightarrow C l(X, x)$ from the divisor class group of $X$ to the local divisor class group $C l(X, x) \cong \mathbb{Z}_{r}$.

We now define the number $m(x, \Delta)$ to be $\operatorname{lcm}\left(m_{i} \mid x \in D_{i}\right)$. Consider the element

$$
m(x, \Delta) \cdot c_{1}(Y / X)=m(x, \Delta)[B]+\sum_{i \mid x \in D_{i}} \frac{m(x, \Delta) b_{i}}{m_{i}} D_{i}
$$

where $c_{1}(Y / X$ is the first Chern class of the Seifert bundle $\pi: Y \longrightarrow X$, see Equation (2.5). Since $m_{i}$ devides $m(x, \Delta)$ for all $i, m(x, \Delta) \cdot c_{1}(Y / X)$ defines an element of the divisor class group $C l(X)$. Kollár [74] gives a criterion for the smoothness of the bundle $\pi: Y \longrightarrow X$.

Theorem 2.53 ([74]). Let $\pi: Y \longrightarrow X$ be the bundle determined by $B$ and $b_{i}$ over the orbifold $\left(X, \Delta=\sum\left(1-\frac{1}{m_{i}}\right) D_{i}\right)$ as in Theorem 2.49. Then $Y$ is smooth along $\pi^{-1}(x)$ if and only if the element

$$
R_{x}\left(m(x, \Delta) \cdot c_{1}(Y / X)\right)
$$

is a generator of the local divisor class group $C l(X, x)$.

We conclude this chapter with a result of Baily on Kähler orbifolds. In this context a Kähler form $\omega$ is a collection of forms $\omega_{i}$ on each chart $\left(\widetilde{U}_{i}, \Gamma_{i}, \varphi_{i}\right)$ satisfying the compatibility conditions. Notice that the Kähler form $\omega$ defines a class in $H^{2}(X ; \mathbb{R}) \cong$ $H_{o r b}^{2}(X ; \mathbb{R})$. We will say that the class $[\omega]$ is integral if it lies in the image of the map $H^{2}(\mathcal{X} ; \mathbb{Z}) \hookrightarrow H^{2}(\mathcal{X} ; \mathbb{R})$. The following theorem of Baily is the orbifold analogue of Kodaira Embedding Theorem.

Theorem $2.54([6])$. Let $\mathcal{X}=(X, \mathcal{U})$ be a Kähler orbifold with integral Kähler class $[\omega]$. Then $X$ is a projective algebraic variety. 


\section{Chapter 3}

\section{Sasaki manifolds}

The aim of this chapter is to give a (partial) overview of Sasakian geometry and topology. Namely, we define Sasaki manifolds and discuss the various underlying structures. We then proceed to prove the Structure Theorem which will lead to most of the topological properties of Sasakian manifolds that we discuss in later chapters. Section 3.7 and Section 3.9 are of particular importance for later chapters. The former is concerned with the transverse geometry of Sasaki structures while the latter gives a brief overview of the topology of Sasakian manifolds.

An introduction to contact and almost contact structures can be found in [9]. Most of the results in this chapter are included in the seminal book of Boyer and Galicki [15]. We refer the reader to [15] for a detailed exposition of the topics in this chapter and Sasakian geometry in general.

\subsection{Contact and almost contact structures}

Definition 3.1. An almost contact structure on a smooth manifold $M$ is a triple $(\eta, \phi, R)$ where $\eta$ is a $1-$ form, $\phi$ is an endomorphism of $T M$ and $R$ is a non-vanishing vector field such that

$$
\eta(R)=1, \quad \phi^{2}=-\mathrm{Id}+R \otimes \eta .
$$

From this definition we can easily derive the following identities:

$$
\phi R=0, \quad \eta \circ \phi=0 .
$$

In order to prove them notice first that $\phi^{2} R=0$. Suppose now $\phi R \neq 0$. From $0=$ $\phi^{2}(\phi R)=-\phi R+\eta(\phi R) R$ we get $\phi R=\eta(\phi R) R \neq 0$. Substituting twice in $0=\phi^{2} R=$ $\eta(\phi R) \phi R=(\eta(\phi R))^{2} R \neq 0$ yields a contradiction. Now given $X \in \mathfrak{X}(M)$ the second identity follows from $\eta(\phi X) R=\phi^{3} X+\phi X=-\phi X+\phi(\eta(X) R)+\phi X=0$. Moreover if $X \in \mathfrak{X}(M)$ satisfies $\phi X=0$ then $0=\phi^{2} X=-X+\eta(X) R$, which proves that $\operatorname{rank}(\phi)=2 n$.

Equivalently we can define almost contact structures as follows. 
Definition 3.2. An almost contact structure on a manifold $M$ of dimension $2 n+1$ is a reduction of the structure group to $U(n) \times 1$.

In order to show that these definitions are indeed equivalent let us introduce some auxiliary structure.

Definition 3.3. An almost contact manifold $(M, \eta, \phi, R)$ is a pair consisting of a smooth manifold $M$ and an almost contact structure $(\eta, \phi, R)$ on $M$. We will sometimes write $M$ for an almost contact manifold and omit the almost contact structure.

Definition 3.4. A Riemannian metric $g$ on an almost contact manifold $(M, \eta, \phi, R)$ is said to be compatible with the almost contact structure if it satisfies

$$
g(\phi X, \phi Y)=g(X, Y)-\eta(X) \eta(Y)
$$

for any two vector fields $X, Y \in \mathfrak{X}(M)$.

For a compatible metric $g$ we have $\eta(X)=g(X, R)$. Notice that any almost contact manifold admits a compatible metric. In fact given any Riemannian metric $g^{\prime}$, a compatible metric $g$ is given by

$$
g(X, Y)=\frac{1}{2}\left(g^{\prime}\left(\phi^{2} X, \phi^{2} Y\right)+g^{\prime}(\phi X, \phi Y)\right)+\eta(X) \eta(Y) .
$$

For future reference we give the following.

Definition 3.5. An almost contact structure $(\eta, \phi, R)$ with a choice of a compatible metric $g$ is called an almost contact metric structure and denoted by $(\eta, \phi, R, g)$.

We can now define an almost contact basis of vector fields in a coordinate chart $\mathcal{U}$. Let $X_{1}$ be a unit vector field orthogonal to $R$. Then $Y_{1}=\phi X_{1}$ is also a unit vector field orthogonal to both $X_{1}$ and $R$. Now choose $X_{2}$ orthogonal to the span of $X_{1}, Y_{1}$ and $R$. Then so is $Y_{2}=\phi X_{2}$. Iterating this process we get a local orthonormal basis $\left\{X_{1}, \ldots, X_{n}, Y_{1}, \ldots, Y_{n}, R\right\}$. Notice that this shows that an almost contact manifold is odd dimensional. Choosing such a basis for each coordinate chart $\mathcal{U}_{\alpha}$ the endomorphism $\phi$ is given locally by

$$
\left(\begin{array}{ccc}
0 & -\mathrm{Id} & 0 \\
\mathrm{Id} & 0 & \vdots \\
0 & \cdots & 0
\end{array}\right) .
$$

Now for $p \in \mathcal{U}_{\alpha} \cap \mathcal{U}_{\beta}$ and $X \in T_{p} M$ let $X_{\alpha}$ and $X_{\beta}$ be the expressions of $X$ in the local basis. In particular we have

$$
X_{\beta}=\left(\begin{array}{ccc}
A & B & 0 \\
C & D & \vdots \\
0 & \cdots & 1
\end{array}\right) X_{\alpha}
$$


where $\left(\begin{array}{cc}A & B \\ C & D\end{array}\right) \in O(2 n)$. Therefore we have

$$
\begin{aligned}
& \left(\begin{array}{ccc}
0 & -\mathrm{Id} & 0 \\
\mathrm{Id} & 0 & \vdots \\
0 & \cdots & 0
\end{array}\right)\left(\begin{array}{ccc}
A & B & 0 \\
C & D & \vdots \\
0 & \cdots & 1
\end{array}\right) X_{\alpha}=\phi\left(X_{\beta}\right)=(\phi X)_{\beta}= \\
= & \left(\begin{array}{ccc}
A & B & 0 \\
C & D & \vdots \\
0 & \cdots & 1
\end{array}\right)\left(\begin{array}{ccc}
0 & -\mathrm{Id} & 0 \\
\mathrm{Id} & 0 & \vdots \\
0 & \cdots & 0
\end{array}\right) X_{\alpha}
\end{aligned}
$$

which shows that $\left(\begin{array}{ll}A & B \\ C & D\end{array}\right)$ commutes with $\left(\begin{array}{cc}0 & - \text { Id } \\ \text { Id } & 0\end{array}\right)$, i.e. $\left(\begin{array}{ll}A & B \\ C & D\end{array}\right) \in U(n)$.

Conversely, if there exists a reduction to $U(n) \times 1$ of the structural group, consider an atlas $\left\{\mathcal{U}_{\alpha}\right\}$ whose transition functions take values in $U(n) \times 1$. Define locally

$$
\phi_{\alpha}=\left(\begin{array}{ccc}
0 & -\mathrm{Id} & 0 \\
\mathrm{Id} & 0 & \vdots \\
0 & \cdots & 0
\end{array}\right), \quad \eta_{\alpha}=\left(\begin{array}{llll}
0 & \cdots & 0 & 1
\end{array}\right) \text { and } R_{\alpha}=\left(\begin{array}{c}
0 \\
\vdots \\
0 \\
1
\end{array}\right) .
$$

Now since $\phi_{\alpha}$ commutes with $U(n) \times 1$ this defines a global endomorphism of $T M$. Moreover, $\eta_{\alpha}$ and $R_{\alpha}$ define $\eta$ and $R$ globally. Finally, the identities

$$
\eta(R)=1, \quad \phi^{2}=-\mathrm{Id}+R \otimes \eta
$$

hold since they are verified in each chart. This shows that Definition 3.1 and Definition 3.2 are equivalent.

An almost contact manifold is equipped with a canonical splitting of the tangent bundle. Namely, the non-vanishing vector field $R$ defines a trivial line bundle $L_{R}$ while $\mathcal{D}=\operatorname{ker} \eta$ defines a codimension 1 sub-bundle of $T M$ with almost complex structure $\phi_{\mid \mathcal{D}}$. Thus the tangent bundle canonically splits as $T M=\mathcal{D} \oplus L_{R}$.

From the very definition of an almost contact structure one gets a topological obstruction for the existence of such structures. In fact if a $2 n+1$-dimensional manifold $M$ admits an almost contact structure then the classifying map $M \rightarrow \mathrm{BS} O(2 n+1)$ of TM factorizes through $\mathrm{BS} U(n)$. The characteristic classes of $M$ are obtained by pulling back those of $S U(n)$. However the odd Stiefel-Whitney classes $w_{2 k+1}$ and integral Stiefel-Whitney classes $W_{k}$ of $S U(n)$ vanish. Thus we get the following classical theorem of Gray.

Theorem 3.6 ([52]). Let $M$ be a $2 n+1$-dimensional almost contact manifold. Then the odd Stiefel-Whitney classes $w_{2 k+1}(M)$ and integral Stiefel-Whitney classes $W_{k}(M)$ vanish. Thus all Stiefel-Whitney numbers vanish and $M$ is the boundary of a compact manifold. 
Let us discuss now equivalence of almost contact structures. Since the notion of equivalence depends on the nature of problem that is studied, we introduce now several relations on almost contact manifolds.

In light of Definition 3.2 we can regard almost contact structures on a $(2 n+1)$ dimensional manifold $M$ as maps from $M$ to $\mathrm{SO}(2 n+1) / \mathrm{U}(n)$. In fact, fix an embedding of $\mathrm{U}(n)$ in $\mathrm{SO}(2 n+1)$. An almost contact structure at a point amounts to an equivalence class of orthonormal frames under the action of $\mathrm{U}(n)$. That is, a section $f: M \longrightarrow$ $\mathrm{SO}(2 n+1) / \mathrm{U}(n)$ of the quotient bundle $\operatorname{Fr}(M) / \mathrm{U}(n)$ of the frame bundle $\operatorname{Fr}(M)$. Then we can define homotopies of almost contact structure as follows.

Definition 3.7. Let $f_{1}: M \longrightarrow \mathrm{SO}(2 n+1) / \mathrm{U}(n)$ and $f_{2}: M \longrightarrow \mathrm{SO}(2 n+1) / \mathrm{U}(n)$ be almost contact structures on a manifold $M$. The two almost contact structures are said homotopic if there exists a homotopy $F: M \times I \rightarrow \mathrm{SO}(2 n+1) / \mathrm{U}(n)$ between $f_{1}$ and $f_{2}$.

Classifying homotopy classes of almost contact structures on arbitrary manifolds is a hard problem. However, in some particular cases the classification can be carried out by means of obstruction theory. We will see such instances in Chapter 5 .

Definition 3.8. Let $\left(M_{1}, \eta_{1}, \phi_{1}, R_{1}\right)$ and $\left(M_{2}, \eta_{2}, \phi_{2}, R_{2}\right)$ be two almost contact manifolds. A diffeomorphism $f: M_{1} \rightarrow M_{2}$ is an isomorphism of almost contact structures if the following two conditions are satisfied

1. $f_{*} \phi_{1}=\phi_{2} f_{*}$,

2. $f^{*} \eta_{2}=g \eta_{1}$ for a non-vanishing function $g \in C^{\infty}\left(M_{1}\right)$.

The two almost contact manifolds are then called isomorphic.

Definition 3.9. Two almost contact structures $\left(\eta_{1}, \phi_{1}, R_{1}\right)$ and $\left(\eta_{2}, \phi_{2}, R_{2}\right)$ on a manifold $M$ are equivalent if they can be identified by a sequence of homotopies and isomorphisms.

Let us now turn our attention to contact structures.

Definition 3.10. A contact form on a manifold $M$ of dimension $2 n+1$ is a 1 -form $\eta$ satisfying $\eta \wedge(\mathrm{d} \eta)^{n} \neq 0$. A contact structure is an equivalence class of contact forms, where two forms $\eta, \eta^{\prime}$ are equivalent if there exists a positive function $f$ such that $\eta=f \eta^{\prime}$.

Remark 3.11. Notice that $\operatorname{ker} \eta=\operatorname{ker} \eta^{\prime}$ if and only if two contact forms $\eta, \eta^{\prime}$ belong to the same equivalence class. Therefore we will identify a contact structure with the distribution $\mathcal{D}=\operatorname{ker} \eta$.

Definition 3.12. A contact manifold $(M, \eta)$ is a pair consisting of a smooth manifold $M$ and a contact form $\eta$ on $M$. By abuse of notation we will often write $M$ for a contact manifold omitting the contact form. 
A choice of a contact form uniquely determines a vector field $R$ by requiring that

$$
\eta(R)=1, \quad \iota_{R} \mathrm{~d} \eta=0 .
$$

To see this notice that the volume form $\eta \wedge(\mathrm{d} \eta)^{n}$ gives an isomorphism of $C^{\infty}(M)$ modules between vector fields on $M$ and $2 n$-forms on $M$. Thus there is a unique vector field $R$ such that $\iota_{R}(\eta \wedge(\mathrm{d} \eta))^{n}=\mathrm{d} \eta^{n}$. Contracting again shows that $\iota_{R}(\mathrm{~d} \eta)^{n}=0$ which in turn implies $\iota_{R} \mathrm{~d} \eta=0$ since $\operatorname{rank}(\mathrm{d} \eta)=2 n$. Moreover, we have

$$
\mathrm{d} \eta^{n}=\iota_{R}(\eta \wedge(\mathrm{d} \eta))^{n}=\eta(R) \mathrm{d} \eta^{n}-\eta \wedge \iota_{R}(\mathrm{~d} \eta)^{n}=\eta(R) \mathrm{d} \eta^{n}
$$

which implies the first identity. The vector field $R$ is called the Reeb vector field of $\eta$ and the foliation determined by the orbits of $R$ is called the characteristic foliation or Reeb foliation of the contact structure.

It is easy to check that the Reeb vector field preserves the contact form nd its exterior derivative, that is,

$$
\mathcal{L}_{R} \eta=\mathcal{L}_{R} \mathrm{~d} \eta=0
$$

In fact we have

$$
\mathcal{L}_{R} \eta=\iota_{R} \mathrm{~d} \eta+\mathrm{d} \iota_{R} \eta=0+\mathrm{d} 1=0 .
$$

Moreover, using $\iota_{R} \mathrm{~d} \eta=0$ we get

$$
\mathcal{L}_{R} \mathrm{~d} \eta=\iota_{R} \mathrm{~d}^{2} \eta+\mathrm{d} \iota_{R} \mathrm{~d} \eta=0 .
$$

Notice that a choice of almost complex structure $J$ on $\mathcal{D}=\operatorname{ker} \eta$ determines an almost contact structure $(\eta, \phi, R)$. In fact, as in the almost contact case we get a canonical splitting of the tangent bundle

$$
T M=\mathcal{D} \oplus L_{R}
$$

where $L_{R}$ is the trivial line bundle given by $R$ and $\mathcal{D}=\operatorname{ker} \eta$ is the contact distribution. Moreover, since $\iota_{R} \mathrm{~d} \eta=0$ and $\operatorname{rank}(\mathrm{d} \eta)=2 n, \mathcal{D}$ is a symplectic sub-bundle with symplectic form $\mathrm{d} \eta$. Then a compatible almost complex structure $J$ on $\mathcal{D}$ can be extended trivially on $L_{R}$ to define $\phi$ on $T M$.

Definition 3.13. A contact form is quasi-regular if each point has a foliated chart for the characteristic foliation such that the intersection with each leaf has at most $k$ connected components and it is irregular otherwise. When $k=1$ the contact form is called regular. By abuse of notation we call a contact manifold $(M, \eta)$ regular (quasi-regular, irregular) if $\eta$ is a regular contact (quasi-regular, irregular) form.

We now state a classical theorem of Darboux which shows that contact structures admit no local invariants.

Theorem 3.14. At each point of a contact manifold $(M, \eta)$ there exist local coordinates $\left(x_{1}, \ldots, x_{n}, y_{1}, \ldots, y_{n}, z\right)$ with respect to which $\eta=d z+\sum_{i=1}^{n} y_{i} d x_{i}$. 
As in the almost contact case we can define several notions of equivalence of contact structures.

Definition 3.15. Let $\left(M_{1}, \eta_{1}\right)$ and $\left(M_{2}, \eta_{2}\right)$ be two contact manifolds. A diffeomorphism $f: M_{1} \rightarrow M_{2}$ is a contactomorphism if $f^{*} \eta_{2}=g \eta_{1}$ for a non-vanishing function $g \in C^{\infty}\left(M_{1}\right)$. The contact manifolds $\left(M_{1}, \eta_{1}\right)$ and $\left(M_{2}, \eta_{2}\right)$ are then called contactomorphic.

One can give a definition of smooth families of contact structures. A celebrated theorem of Gray shows that this is in fact equivalent to an isotopy.

Theorem 3.16 ([52]). Let $M$ be a closed contact manifold. Assume $\mathcal{D}_{t}$ is a smooth family of contact structures for $t \in[0,1]$. Then there is an isotopy $\psi_{t}$ of $M$ with $t \in[0,1]$ such that

$$
\left(\psi_{t}\right)_{*} \mathcal{D}_{0}=\mathcal{D}_{t} \text { for all } t \in[0,1] .
$$

Remark 3.17. Theorem 3.16 does not hold for a smooth deformation of contact forms $\eta_{t}$.

In parallel with the almost contact case we give the following:

Definition 3.18. Two contact structures $\mathcal{D}_{1}$ and $\mathcal{D}_{2}$ on a closed manifold $M$ are equivalent if they can be identified by a sequence of isotopies and contactomorphisms.

We present now several examples of contact structures, some of which will turn out to be fundamental examples of Sasaki structures.

Example 3.19 (Standard contact structure on $S^{2 n+1}$ ). Consider $S^{2 n+1} \subset \mathbb{C}^{n+1}$ with standard coordinates $z=\left(z_{0}, \ldots, z_{n}\right)$ where $z_{j}=x_{j}+i y_{j}$. The restriction of $\eta_{0}=$ $\sum_{j=0}^{n}\left(x_{j} \mathrm{~d} y_{j}-y_{j} \mathrm{~d} x_{j}\right)$ to $S^{2 n+1}$ is a contact form. In order to see that consider the form $\alpha=\sum_{j=0}^{n}\left(x_{j} \mathrm{~d} x_{j}+y_{j} \mathrm{~d} y_{j}\right)$ normal to the unit sphere. A simple computation shows that

$$
\alpha \wedge \eta_{0} \wedge \mathrm{d} \eta_{0}^{n}=2^{n} n ! \sum_{j=0}^{n}\left(x_{j}^{2}+y_{j}^{2}\right) \mathrm{d} x_{0} \wedge \mathrm{d} y_{0} \wedge \cdots \wedge \mathrm{d} x_{n} \wedge \mathrm{d} y_{n}
$$

therefore $\eta_{0} \wedge \mathrm{d} \eta_{0}^{n}$ is a volume form on $S^{2 n+1}$.

In other words if $N$ is the unit normal to the sphere and $\omega=\sum_{j=0}^{n} \mathrm{~d} x_{j} \wedge \mathrm{d} y_{j}$ is the standard Kähler form then $\eta_{0}=\iota_{N} \omega$. It is easy to check that if $J$ is the standard complex structure on $\mathbb{C}^{n+1}$ then $J N=R_{0}$ is the Reeb vector field.

Example 3.20. The previous example provides a contact structure on odd dimensional real projective spaces since the standard structure on $S^{2 n+1}$ is invariant under the reflection $z \mapsto-z$.

Example 3.21. It was proven by Bourgeois [14] that all odd dimensional tori admit contact structures. Even though it is not trivial to show this in full generality, one can easily give an explicit contact structure on $T^{3}=\mathbb{R}^{3} / \mathbb{Z}^{3}$. In fact the 1 -form $\eta=\sin y \mathrm{~d} x+$ $\cos y \mathrm{~d} z$ is such that $\eta \wedge \mathrm{d} \eta=-\mathrm{d} x \wedge \mathrm{d} y \wedge \mathrm{d} z$ in the standard coordinates $(x, y, z)$ of $\mathbb{R}^{3}$. 


\subsection{The Boothby-Wang fibration}

In this section we introduce a construction that will be crucial in the rest of the thesis. Namely, we present a special class of contact manifolds, called Boothby-Wang fibrations, which arise as principal $S^{1}$-bundles over symplectic manifolds.

Let $X$ be a smooth manifold and $\pi: M \longrightarrow X$ a principal $S^{1}$-bundle. Suppose $\omega$ is a 2-form representing the first Chern class of $M$ in $H^{2}(X ; \mathbb{Z})$. Any connection 1-form $\eta^{\prime}$ satisfies $\mathrm{d} \eta^{\prime}=-2 \pi i \pi^{*} \omega^{\prime}$ for some 2-form $\omega^{\prime}$ representing $[\omega]$. Now consider $\beta$ to be a 1 -form on $X$ such that $\mathrm{d} \beta=\omega-\omega^{\prime}$ and set $\eta=\eta^{\prime}-2 \pi i \pi^{*} \beta$. The form $\eta$ is a connection 1-form on $M$ since $\pi^{*} \beta$ is horizontal and invariant. Moreover, we have

$$
\begin{aligned}
\mathrm{d} \eta & =\mathrm{d} \eta^{\prime}-2 \pi i \mathrm{~d} \pi^{*} \beta=-2 \pi i \pi^{*} \omega^{\prime}-2 \pi i \pi^{*} \mathrm{~d} \beta \\
& =-2 \pi i \pi^{*} \omega^{\prime}-2 \pi i \pi^{*}\left(\omega-\omega^{\prime}\right) \\
& =-2 \pi i \pi^{*} \omega .
\end{aligned}
$$

We have shown that on the principal $S^{1}$-bundle associated to $[\omega]$ one can choose a connection 1-form $\eta$ such that $\mathrm{d} \eta=-2 \pi i \pi^{*} \omega$.

Notation. The first Chern class $c_{1}(M)=[\omega] \in H^{2}(X ; \mathbb{Z})$ associated to the principal $S^{1}$-bundle $M \stackrel{\pi}{\rightarrow} X$ coincide with the Euler class of the associated rank 2 vector bundle bundle. Therefore, we will not distinguish between the two.

Notation. In order to lighten the notation we identify the Lie algebra $i \mathbb{R}$ of $U(1)$ with $\mathbb{R}$ and drop the complex notation. In the same spirit we omit the coefficient $2 \pi$.

The following theorem of Boothby and Wang gives a one-to-one correspondence between regular contact structures and non-trivial principal circle bundles over symplectic manifolds with integral symplectic class.

Theorem 3.22 ([11] $)$. Let $\left(M, \eta^{\prime}\right)$ be a compact regular contact manifold. Then there exists a non-vanishing function $f$ such that the Reeb vector field $R$ associated to the contact form $\eta=f \eta^{\prime}$ generates a free $S^{1}$-action on $M$. Moreover, the orbits of $R$ are the fibers of a principal $S^{1}$-bundle $\pi: M \rightarrow X$ over a symplectic manifold $(X, \omega)$ such that $\eta$ is a connection form with curvature form $d \eta=\pi^{*} \omega$.

Conversely, if $(X, \omega)$ is a symplectic manifold such that the class $[\omega]$ is integral, then the principal $S^{1}$-bundle $M$ associated to $[\omega]$ is a regular contact manifold with contact form $\eta$ such that $d \eta=\pi^{*} \omega$.

Proof. Since the contact structure is regular and $M$ is compact, the leaves of the characteristic foliation are homeomorphic to circles. Moreover, again by regularity of the characteristic foliation, $M$ is a fiber bundle over a smooth manifold $X$.

Let $\varphi^{\prime}$ be the flow of the Reeb vector field $R^{\prime}$ of $\eta^{\prime}$ and $f$ be its period map, i.e. $f(p)=\min \left\{t \in \mathbb{R} \mid \varphi_{t}^{\prime}(p)=p\right\}$. The map $f$ is constant along orbits of $R^{\prime}$ and it is positive and finite since there are no fixed points and the leaves are circles. We now show 
that $f$ is constant on $M$. Denote the projection by $\pi: M \rightarrow X$ and define a compatible metric by $g=\pi^{*} h+\eta^{\prime} \otimes \eta^{\prime}$ where $h$ is a metric on $X$. Now $R^{\prime}$ is a Killing vector field for $g$ since $\eta^{\prime}\left(R^{\prime}\right)=1$ and $\mathcal{L}_{R^{\prime}} \eta^{\prime}=0$. Moreover, its orbits are geodesics because $g\left(\nabla_{R^{\prime}} R^{\prime}, V\right)=-g\left(\nabla_{V} R^{\prime}, R^{\prime}\right)=0$ where $\nabla$ is the Levi-Civita connection of $g$. Let $\gamma$ be the orbit through $p$ and $\gamma^{\prime}$ a sufficiently close orbit. Then there is a unique minimal geodesic from $p$ to $\gamma^{\prime}$ and it meets $\gamma$ and $\gamma^{\prime}$ orthogonally. Denote by $\delta$ the geodesic arc between $p$ and $\gamma^{\prime}$. Now since $\varphi^{\prime}$ acts by isometries the image of $\delta$ is orthogonal to both $\gamma$ and $\gamma^{\prime}$ at all times. Therefore, when $p$ moves by one period on $\gamma$, the endpoint of $\delta$ also moves by one period. Thus the function $f$ is locally constant, therefore constant, on $M$.

Now define $\eta=\frac{1}{f} \eta^{\prime}$ and $R=f R^{\prime}$. Notice that $R$ is the Reeb vector field of $\eta$ since $f$ is constant. Moreover, the period function of $R$ is identically 1 thus the action of its flow $\varphi_{t}$ only depends on $t \bmod 1$. Therefore $R$ induces a free $S^{1}$-action on $M$.

Recall that $\mathcal{L}_{R} \eta=\mathcal{L}_{R} \mathrm{~d} \eta=0$ so that both $\eta$ and $\mathrm{d} \eta$ are invariant forms. Now, having identified the Lie algebra of $S^{1}$ with $\mathbb{R}$, we can regard $\eta$ as the connection 1form associated to the Ehresmann connection $\mathcal{D}=\operatorname{ker} \eta$. Since $S^{1}$ is abelian we get that $\mathrm{d} \eta$ is the curvature form for $\eta$. Now $\mathrm{d} \eta$ is horizontal and invariant therefore there exists a 2-form $\omega$ on $X$ such that $\pi^{*} \omega=\mathrm{d} \eta$. Moreover $\pi^{*} \mathrm{~d} \omega=\mathrm{d} \pi^{*} \omega=\mathrm{d}^{2} \eta=0$ implies $\mathrm{d} \omega=0$. Thus $\omega$ is closed and defines an integral cohomology class on $X$. Furthermore $X$ is symplectic because $\mathrm{d}\left(\omega^{n}\right)=\mathrm{d} \omega^{n}=\mathrm{d} \eta^{n} \neq 0$ yields $\omega^{n} \neq 0$.

Conversely, let $(X, \omega)$ be a $2 n$-dimensional symplectic manifold such that the symplectic form $\omega$ represents an integral class $[\omega] \in H^{2}(X ; \mathbb{Z})$. Let $\pi: M \rightarrow X$ be the principal $S^{1}$-bundle associated to $[\omega]$. Then, as explained in the beginning of this section, there exists a connection form $\eta$ such that $\mathrm{d} \eta=\pi^{*} \omega$. Denote by $R$ a vertical vector field such that $\eta(R)=1$ and let $V_{1}, \ldots, V_{2 n}$ be linearly independent horizontal vector fields. Then $\eta \wedge(\mathrm{d} \eta)^{n}=\eta(R) \mathrm{d} \eta^{n}\left(V_{1}, \ldots, V_{2 n}\right) \neq 0$ and we can regard $\eta$ as a contact form by identifying the Lie algebra of $S^{1}$ with $\mathbb{R}$. Moreover the contact structure on $M$ is regular by construction.

Notation. In the setting of Theorem 3.22 the regular contact manifold $M$ is called the Boothby-Wang fibration, or Boothby-Wang construction, over $(X, \omega)$.

Remark 3.23. In general we can associate (not uniquely) a Boothby-Wang fibration to any symplectic manifold $(X, \Omega)$ regardless of whether or not $[\Omega]$ is integral. Namely, let $g$ be any metric on $X$. Consider a ball $B_{\epsilon}$ of radius $\epsilon$ around the origin of the space of harmonic 2-forms on $X$ with respect to $g$. Since non-degeneracy is an open condition, for $\epsilon$ small enough every form in $\Omega+B_{\epsilon}$ is symplectic. Moreover, $\Omega+B_{\epsilon}$ represents an open set of classes in $H^{2}(X ; \mathbb{R})$. Therefore we can choose a form $\Omega^{\prime}$ in $\Omega+B_{\epsilon}$ such that $\left[\Omega^{\prime}\right]$ lies in the image of the map $H^{2}(X ; \mathbb{Q}) \longrightarrow H^{2}(X ; \mathbb{R})$, i.e. such that $\left[\Omega^{\prime}\right]$ is a rational class. Thus a suitable multiple $\omega$ of $\Omega^{\prime}$ represents an integral class $[\omega] \in H^{2}(X ; \mathbb{Z})$. Now Theorem 3.22 provides a contact manifold $(M, \eta)$ which is a principal $S^{1}$-bundle over $X$. Notice that the choice of $\Omega^{\prime}$ is not canonical. Hence a different choice can lead to a different principal $S^{1}$-bundle $M^{\prime} \longrightarrow X$. 
Example 3.24. The most classical example of a Boothby-Wang fibration is the Hopf fibration of odd-dimensional spheres. These are indeed $S^{1}$-bundles associated to the generator $\left[\omega_{F S}\right]$ of $H^{2}\left(\mathbb{C P}^{n}, \mathbb{Z}\right)$ and give the standard contact structure on spheres discussed in Example 3.19. In order to see this consider the Hopf fibration, i.e. the restriction to $S^{2 n+1}$ of the fibration $\mathbb{C}^{*} \rightarrow \mathbb{C}^{n+1} \backslash\{0\} \stackrel{\pi}{\rightarrow} \mathbb{C P}^{n}$. Now the Reeb vector field $R_{0}=J N$ is in $\operatorname{ker}\left(\pi_{*}\right)$ because $N \in \operatorname{ker}\left(\pi_{*}\right)$ and the kernel is a complex line. Therefore, the orbits of $R_{0}$ are the fibers of the fibration which, in particular, assures that $\eta_{0}$ defines a regular contact structure. Now an orbit of $R_{0}$ is a maximal circle on the sphere. Thus its period is $2 \pi$ because $R_{0}$ has constant norm 1 . Therefore the contact form $\eta_{0}$ defines a connection form on the principal $S^{1}$-bundle $S^{2 n+1} \stackrel{\pi}{\rightarrow} \mathbb{C P}^{n}$ such that $\pi^{*} \omega_{F S}=\mathrm{d} \eta_{0}$.

\subsection{K-contact structures}

We have introduced the concept of metrics compatible with an almost contact structure in Definition 3.5. In the remainder of this chapter we will discuss contact structures endowed with such metrics satisfying some additional properties.

As seen before, one can associate an almost contact structure $(\eta, \phi, R)$ to a given contact form $\eta$ in the following way. Fix an almost complex structure $J$ on $\mathcal{D}=\operatorname{ker} \eta$ compatible with $\mathrm{d} \eta$. The extension $\phi$ to $\mathrm{T} M$ given by setting $\phi R=0$ satisfies $\phi^{2}=$ $-\mathrm{Id}+R \otimes \eta$. Therefore, the triple $(\eta, \phi, R)$, given by the contact form $\eta$, the Reeb vector field $R$ and the endomorphism $\phi$, is an almost contact structure.

Remark 3.25. Notice that the almost contact structure so induced depends on the choice of $J$ while its homotopy class does not since the space of almost complex structures compatible with $\mathrm{d} \eta$ is contractible.

Definition 3.26. Let $(M, \eta)$ be a contact manifold. An almost contact (metric) structure $\left(\eta^{\prime}, \phi, R^{\prime}, g\right)$ is compatible with the contact structure if $\eta^{\prime}=\eta, R^{\prime}$ is the Reeb vector field $R$ of $\eta$ and $\phi$ satisfies

$$
\mathrm{d} \eta(\phi X, \phi Y)=\mathrm{d} \eta(X, Y), \quad \mathrm{d} \eta(\phi X, X)>0 .
$$

This is equivalent to the compatibility of the almost complex structure $\phi_{\mid \mathcal{D}}$ with $\mathrm{d} \eta$. Moreover, given a contact manifold $(M, \eta)$, every compatible almost contact structure is uniquely determined by $\phi_{\mid \mathcal{D}}$.

For an almost contact structure $(\eta, \phi, R)$ it is possible to define a unique metric $g$ such that $g(X, \phi Y)=\mathrm{d} \eta(X, Y)$. Such a metric is called the associated metric to $(\eta, \phi, R)$.

Definition 3.27. A contact metric structure $(\eta, \phi, R, g)$ is given by a contact structure $\eta$, with a compatible almost contact metric structure $(\eta, \phi, R, g)$ such that $g(X, \phi Y)=$ $\mathrm{d} \eta(X, Y)$. A manifold endowed with such a structure is called a contact metric manifold. 
Remark 3.28. Notice that a contact metric structure does not impose any restriction on the topology of the underlying manifold in addition to those seen in Theorem 3.6 since every contact structure $\eta$ admits a compatible almost contact metric structure $(\eta, \phi, R, g)$ such that $g(X, \phi Y)=\mathrm{d} \eta(X, Y)$.

In order to see this let $\eta$ be a contact form on a manifold $M$. Then $\mathcal{D}=\operatorname{ker} \eta$ is a symplectic distribution endowed with the symplectic form $\mathrm{d} \eta$. Fix an almost complex structure $J$ on $\mathcal{D}$ which is compatible with $\mathrm{d} \eta$. We can extend $J$ to an endomorphism $\phi$ of $T M$ by requiring that $\phi R=0$. This defines an almost contact structure $(\eta, \phi, R)$. The associated metric $g$ makes $(\eta, \phi, R, g)$ a contact metric structure by definition.

Therefore, we can regard a contact metric structure $(\eta, \phi, R, g)$ as a contact form $\eta$ with a choice of compatible almost complex structure $J$ on $\mathcal{D}$.

Definition 3.29. A K-contact structure $(\eta, \phi, R, g)$ is a contact metric structure such that $R$ is a Killing vector field of $g$. A manifold with such a structure is a K-contact manifold.

Notation. We will use the terminology K-contact manifold both for a manifold endowed with a $K$-contact structure and for a manifold admitting a $K$-contact structure. The meaning will be clear from the context.

Several different structures with suitable compatibility conditions come together in the definition of K-contact manifold. The abundance of underlying structures allows several equivalent definitions of K-contact manifolds. Let us then discuss some equivalent definitions of K-contact manifolds.

Very often K-contact structures are referred to as contact structures whose Reeb flow preserves a transverse almost Kähler structure. Let us explain this phrasing. The term transverse refers to the Reeb foliation. A contact form $\eta$ has a certain transverse geometric structure if this structure is transverse to the Reeb foliation. In this setting an almost Kähler structure transverse to the characteristic foliation is induced naturally by a contact metric structure. Namely, the restriction $\phi_{\left.\right|_{\mathcal{D}}}$ of the endomorphism $\phi$ to the contact distribution defines an almost complex structure $J$ on $\mathcal{D}$ compatible with the symplectic form $\mathrm{d} \eta$.

As discussed in Remark 3.28, given a contact form $\eta$ we can always define a transverse almost Kähler structure induced by a contact metric structure $(\eta, \phi, R, g)$. However, invariance under the Reeb flow is a non-trivial condition. The canonical transverse almost Kähler structure is preserved by $R$ if and only if the flow of $R$ preserves $\mathrm{d} \eta, \phi$ and $g$. Now the Reeb vector field preserves $\mathrm{d} \eta$ by definition. Moreover, the metric is given by $g=\mathrm{d} \eta \circ(\phi \otimes \mathrm{Id})$. Hence $g$ is invariant under the flow of $R$ if and only if so is $\phi$, see also the proof of Proposition 3.41. We have shown the following equivalences for a contact manifold $(M, \eta, \phi, R, g)$ :

$$
\mathcal{L}_{R} g=0 \Longleftrightarrow \mathcal{L}_{R} \phi=0 \Longleftrightarrow \mathrm{K} \text {-contact } \Longleftrightarrow R \text { preserves transverse a.K.s. . }
$$

Hence one can regard K-contact structures as contact metric structures whose endomorphism $\phi$ or metric $g$ are invariant under the flow of $R$. 
Furthermore, we can rephrase these conditions in terms of the almost complex and symplectic structures transverse to the characteristic foliation. The choice of a compatible almost complex structure $J$ on $\mathcal{D}$ determines the endomorphism $\phi$ and the associated metric $g$ such that $(\eta, \phi, R, g)$ is a contact metric structure. Conversely, since $\mathrm{d} \eta$ is a symplectic form on $\mathcal{D}$, the choice of a transversal metric $g_{\mathcal{D}}$ on $\mathcal{D}$ determines a compatible almost complex structure $J$. Thus we get a contact metric structure $(\eta, \phi, R, g)$ by requiring that $R$ is a normal vector orthogonal to $\mathcal{D}$ and extending $J$ to $T M$ trivially. Moreover, it is clear that

$$
\mathcal{L}_{R} \phi=0 \Longleftrightarrow \mathcal{L}_{R} J=0, \quad \mathcal{L}_{R} g=0 \Longleftrightarrow \mathcal{L}_{R} g_{\mathcal{D}}=0
$$

Hence we can rewrite the conditions above as

$$
\mathcal{L}_{R} g_{\mathcal{D}}=0 \Longleftrightarrow \mathcal{L}_{R} J=0 \Longleftrightarrow \text { K-contact . }
$$

Notice that the choice of a compatible almost complex structure $J$ defines a transverse almost Kähler structure of the form $\left(\mathrm{d} \eta, J, g_{\mathcal{D}}\right)$ where $g_{\mathcal{D}}$ is determined by the compatibility condition. Hence a K-contact structure can be seen as a contact form with the choice of an $R$-invariant transverse almost Kähler structure of the form $\left(\mathrm{d} \eta, J, g_{\mathcal{D}}\right)$.

We summarize the discussion above in the following:

Definition 3.30. Let $(M, \eta)$ be a contact manifold. A K-contact structure on $(M, \eta)$ is given by one of the following:

i) A contact metric structure $(\eta, \phi, R, g)$ such that $R$ is Killing for $g$, i.e. $\mathcal{L}_{R} g=0$.

ii) A contact metric structure $(\eta, \phi, R, g)$ such that the flow of $R$ preserves $\phi$, i.e. $\mathcal{L}_{R} \phi=0$.

iii) The choice of a compatible almost complex structure $J$ on $\mathcal{D}=\operatorname{ker} \eta$ such that the flow of $R$ preserves $J$, i.e. $\mathcal{L}_{R} J=0$.

iv) The choice of a transverse metric $g_{\mathcal{D}}$ on $\mathcal{D}=\operatorname{ker} \eta$ such that the flow of $R$ preserves $g_{\mathcal{D}}$, i.e. $\mathcal{L}_{R} g_{\mathcal{D}}=0$.

v) A transverse almost Kähler structure $\left(\mathrm{d} \eta, J, g_{\mathcal{D}}\right)$ which is preserved by the flow of $R$.

Example 3.31 (Standard sphere). In Example 3.19 we have presented the standard contact structure on the odd dimensional sphere $S^{2 n+1}$. Identifying $\mathbb{R}^{2 n+2}$ with $\mathbb{C}^{n+1}$ with the complex structure given by $J \partial_{x_{j}}=\partial_{y_{j}}$ we can describe the contact distribution as $\mathrm{TS}^{2 n+1} \cap J \mathrm{TS}^{2 n+1}=\operatorname{ker} \eta$. Therefore, $J$ restricts to an almost complex structure on $\mathcal{D}=\operatorname{ker} \eta$ and the round metric on the sphere induced by the Euclidian metric on $\mathbb{R}^{2 n+2}$ is compatible with the contact structure. We have shown that the standard contact structure on $S^{2 n+1}$ with the round metric is a contact metric structure. We show that this structure is indeed K-contact in the following example. 
Example 3.31 above is an instance of a larger class of K-contact manifolds that we have already encountered, namely Boothby-Wang fibrations. We now show that all such manifolds are K-contact.

Proposition 3.32. A Boothby-Wang bundle over an almost Kähler manifold has a canonical $K$-contact structure. Equivalently, a regular contact manifold is $K$-contact. Conversely, every regular $K$-contact manifold is a Boothby-Wang bundle over an almost Kähler manifold.

Proof. Let $(M, \eta)$ be a regular contact manifold of dimension $2 n+1$ and $\pi: M \rightarrow X$ its Boothby-Wang fibration. Then $X$ has an integral symplectic form $\omega$ such that $\pi^{*} \omega=\mathrm{d} \eta$. Since $\eta$ is a connection form for the principal $S^{1}$-bundle $M$, it defines a horizontal lift, say $\tilde{\pi}$. Now let $J$ be an almost complex structure compatible with $\omega$ and $h$ the associated metric on $X$. We can define a tensor $\phi$ on $M$ by $\phi V=\tilde{\pi}\left(J \pi_{*}(V)\right)$. Since the Reeb vector field $R$ is vertical we have $\phi^{2}=-\tilde{\pi} \circ \pi_{*}=-\mathrm{Id}+\eta \otimes R$. Therefore $(\eta, \phi, R)$ is a compatible almost contact structure. Now for the metric on $M$ defined by $g=\pi^{*} h+\eta \otimes \eta$ we have

$$
g(V, \phi W)=h\left(\pi_{*} V, J \pi_{*} W\right) \circ \pi=\omega\left(\pi_{*} V, \pi * W\right) \circ \pi=\pi^{*} \omega(V, W)=\mathrm{d} \eta(V, W) .
$$

Thus $(\eta, \phi, R, g)$ defines a contact metric structure on $M$. Moreover, it is clear that $R$ is a Killing vector field because $\pi^{*} h$ is invariant under the $S^{1}$-action and $\mathcal{L}_{R} \eta=0$ by definition of $R$. We have shown that a Boothby-Wang fibration admits a K-contact structure which depends on the choice of an almost Kähler structure on the base.

Conversely, a regular K-contact structure define a Boothby-Wang bundle by Theorem 3.22. Moreover, the endomorphism $\phi$ restricts to a transverse almost complex structure compatible with $\mathrm{d} \eta$. Therefore, it induces an almost Kähler structure on the base of the Boothby-Wang fibration.

Remark 3.33. An alternative proof of the fact that a regular contact manifold is Kcontact is given by averaging an associated metric over the $S^{1}$-action. Namely, given a regular contact structure $\eta$ take any contact metric structure $\left(\eta, \phi, R, g^{\prime}\right)$ and replace $g^{\prime}$ by

$$
g=\int_{S^{1}} \varphi_{t}^{*} g^{\prime} \mathrm{d} t
$$

where $\varphi_{t}$ is the flow of $R$.

As discussed in Remark 3.28, given a contact manifold $(M, \eta)$ the existence of a contact metric structure on $(\eta, \phi, R, g)$ does not restrict further the topology of $M$. Nevertheless, the existence of K-contact structures is, in general, obstructed by the topology of $M$. The following theorems will give us such restrictions and will enable us to find examples of contact manifolds admitting contact structures but no K-contact structures.

Theorem 3.34 ([110]). Let $\pi: M \rightarrow X$ be the Boothby-Wang fibration associated to a compact regular contact manifold $(M, \eta)$. Then $b_{1}(M)=b_{1}(X)$. 
Proof. Let $e=[\omega]$ be the Euler class of the $S^{1}$-bundle $\pi: M \rightarrow X$. The Gysin sequence of the bundle reads

$$
0 \rightarrow H^{1}(X ; \mathbb{R}) \stackrel{\pi^{*}}{\longrightarrow} H^{1}(M ; \mathbb{R}) \rightarrow H^{0}(X ; \mathbb{R}) \stackrel{\cdot v e}{\longrightarrow} H^{2}(X ; \mathbb{R}) \rightarrow \cdots
$$

Since $\omega$ is a symplectic form the last map $\cup e$ is injective and the pullback map $H^{1}(X ; \mathbb{R}) \stackrel{\pi^{*}}{\longrightarrow} H^{1}(M ; \mathbb{R})$ is an isomorphism.

Corollary 3.35. The torus $T^{2 n+1}$ does not admit a regular contact structure.

Proof. Assume $T^{2 n+1}$ admits a regular contact structure and let $T^{2 n+1} \rightarrow X$ be the associated Boothby-Wang fibration. The long homotopy sequence of the fibration becomes

$$
0 \rightarrow \pi_{2}(X) \rightarrow \pi_{1}\left(S^{1}\right) \rightarrow \pi_{1}\left(T^{2 n+1}\right) \rightarrow \pi_{1}(X) \rightarrow 0
$$

because $\pi_{k}\left(S^{1}\right)=\pi_{k}\left(T^{2 n+1}\right)=0$ for all $k>1$. Now consider the lift of the fibration to the universal cover $\mathbb{R}^{2 n+1}$. Here the leaves lift to lines therefore the fibration does not have nullhomotopic fibers. Thus the map $\pi_{1}\left(S^{1}\right) \rightarrow \pi_{1}\left(T^{2 n+1}\right)$ is non-trivial. This, in turn, implies that $\pi_{2}(X)=0$ since $\pi_{1}\left(T^{2 n+1}\right)$ is torsion free. Hence $\pi_{1}(X)=\mathbb{Z}^{2 n+1} / \mathbb{Z}$ and $b_{1}(X)=2 n$ which is a contradiction to Theorem 3.34

We can actually prove that tori cannot support a K-contact structure, being it regular or not. This is because the existence of a K-contact structure constrains the topology of $M$. Before we make this statement precise let us prove the following:

Lemma 3.36. Let $(M, \eta, \phi, R, g)$ be a compact $K$-contact manifold. If $\alpha$ is a harmonic 1-form then $\alpha(R)=0$.

Proof. Let $f=\alpha(R)$ and decompose $\alpha$ into $\alpha=\beta+f \eta$. Since harmonic forms are invariant under isometries and $R$ is Killing one gets

$$
0=\mathcal{L}_{R} \alpha=\mathrm{d} \iota_{R} \alpha+\iota_{R} \mathrm{~d} \alpha=\mathrm{d} \iota_{R} \alpha=\mathrm{d} f
$$

so that $f$ is constant. Thus $0=\mathrm{d} \alpha=\mathrm{d} \beta+f \mathrm{~d} \eta$. By Stokes' Theorem we have

$$
0=\int_{M} \mathrm{~d}\left(\beta \wedge \eta \wedge \mathrm{d} \eta^{n-1}\right)=-\int_{M} f \eta \wedge \mathrm{d} \eta^{n}
$$

which implies the claim since $\eta \wedge \mathrm{d} \eta^{n}$ is a volume form.

The following result was first proved by Rukimbira [101] in a slightly different, although equivalent, setting and later by Itoh [63] in the K-contact setting.

Theorem 3.37 ([101, 63]). Let $(M, \eta, \phi, R, g)$ be a compact $K$-contact manifold of dimension $2 n+1$. Then the cup length cup $(M)$ of $M$ satisfies

$$
1 \leqslant \operatorname{cup}(M) \leqslant 2 n
$$


Proof. By Hodge theory we can represent every cohomology class by a harmonic form. If the cup length of $M$ equals $2 n+1$, we can write a non-trivial class $c \in H^{2 n+1}(M ; \mathbb{R})$ as the cup product of $2 n+1$ harmonic 1 -forms $\alpha_{1} \wedge \cdots \wedge \alpha_{2 n+1}$. Now we complete $R$ to a local basis $\left\{R, v_{1}, \ldots, v_{2 n}\right\}$. By Lemma $3.36 \alpha_{1} \wedge \cdots \wedge \alpha_{2 n+1}\left(R, v_{1}, \ldots, v_{2 n}\right)=0$. Therefore $c \in H^{2 n+1}(M ; \mathbb{R})$ is the trivial class contradicting the assumption.

Corollary 3.38. Let $\Sigma_{g}$ be a compact orientable surface of genus $g \geqslant 1$. Then the manifold $\Sigma_{g_{1}} \times \cdots \times \Sigma_{g_{k}} \times S^{1}$ does not admit a $K$-contact structure (hence it does not admit a regular contact structure). In particular, tori do not admit $K$-contact structures.

\subsection{Complex structures on the Riemannian cone}

Let us take a step back and consider again almost contact manifolds. We now draw a parallel between almost contact structures and almost complex structures. In this light we will define an odd dimensional counterpart of complex structures. Namely, consider an almost contact structure $(M, \eta, \phi, R)$ and its cone $M \times \mathbb{R}^{+}$. Let $\partial_{t}$ be the vector tangent to the second factor and define an automorphism of the tangent bundle by

$$
I X=\phi X+\eta(X) \partial_{t}, \quad I \partial_{t}=-R
$$

where $X \in \mathfrak{X}(M)$. Since $I^{2}=-\mathrm{Id}$, the almost contact structure $(\eta, \phi, R)$ defines an almost complex structure $I$ on $M \times \mathbb{R}^{+}$. This is called the almost complex structure associated to $(\eta, \phi, R)$. By analogy, an almost contact manifold $(M, \eta, \phi, R)$ which induces an integrable almost complex structure $I$ on $M \times \mathbb{R}^{+}$can be considered the odd dimensional analogue of a complex manifold.

Definition 3.39. An almost contact structure $(\eta, \phi, R)$ on a manifold $M$ is called normal if the induced almost complex structure $I$ on the cone $M \times \mathbb{R}^{+}$is integrable.

By a classical result of Newlander and Nirenberg, an almost complex structure $J$ is integrable if and only if the Nijenhuis tensor $N_{J}(X, Y)=J^{2}[X, Y]+[J X, J Y]-J[J X, Y]-$ $J[X, J Y]$ vanishes. We compute the tensor $N_{I}$ in order to express the integrability of $I$ in terms of the almost contact structure $(\eta, \phi, R)$. Since $N_{I}$ is a tensor on $M \times \mathbb{R}^{+}$it suffices 
to compute it for pairs of the form $(X, Y)$ and $\left(X, \partial_{t}\right)$ with $X, Y \in \mathfrak{X}(M)$.

$$
\begin{aligned}
N_{I}(X, Y)= & I^{2}[X, Y]+[I X, I Y]-I[I X, Y]-I[X, I Y] \\
& -[X, Y]+\left[\phi X+\eta(X) \partial_{t}, \phi Y+\eta(Y) \partial_{t}\right] \\
& -I\left[\phi X+\eta(X) \partial_{t}, Y\right]-I\left[X, \phi Y+\eta(Y) \partial_{t}\right] \\
= & \phi^{2}[X, Y]-\eta([X, Y]) R+[\phi X, \phi Y]+(\phi X(\eta(Y))-\phi Y(\eta(X))) \partial_{t} \\
& -I[\phi X, Y]+I Y(\eta(X)) \partial_{t}-I[X, \phi Y]-I X(\eta(Y)) \partial_{t} \\
= & \phi^{2}[X, Y]-\eta([X, Y]) R+[\phi X, \phi Y]+(\phi X(\eta(Y))-\phi Y(\eta(X))) \partial_{t} \\
& -\phi[\phi X, Y]-\phi[X, \phi Y]-(\eta([\phi X, Y])+\eta([X, \phi Y])) \partial_{t} \\
& +(X(\eta(Y))-Y(\eta(X))) R \\
= & N_{\phi}(X, Y)+2 \mathrm{~d} \eta(X, Y) R+2 \mathrm{~d} \eta(\phi X, Y) \partial_{t}+2 \mathrm{~d} \eta(X, \phi Y) \partial_{t} \\
= & N_{\phi}(X, Y)+2 \mathrm{~d} \eta(X, Y) R-2\left(\left(\mathcal{L}_{\phi X} \eta\right)(Y)-\left(\mathcal{L}_{\phi Y} \eta\right)(X)\right) \partial_{t} .
\end{aligned}
$$

Similarly we have

$$
\begin{aligned}
N_{I}\left(X, \partial_{t}\right) & =I^{2}\left[X, \partial_{t}\right]+\left[I X, I \partial_{t}\right]-I\left[I X, \partial_{t}\right]-I\left[X, I \partial_{t}\right] \\
& =-\left[\phi X+\eta(X) \partial_{t}, R\right]-I\left[\phi X+\eta(X) \partial_{t}, \partial_{t}\right]+I[X, R] \\
& =-[\phi X, R]+R(\eta(X)) \partial_{t}+\phi[X, R]+\eta([X, R]) \partial_{t} \\
& =-[\phi X, R]+\phi[X, R]+(\eta([X, R])+R(\eta(X))) \partial_{t} \\
& =\left(\mathcal{L}_{R} \phi\right)(X)+\left(\mathcal{L}_{R} \eta\right)(X) \partial_{t} .
\end{aligned}
$$

Separating the components tangent to the two factors, we define the following tensors on $M$ :

$$
\begin{aligned}
N^{(1)}(X, Y) & =N_{\phi}(X, Y)+2 \mathrm{~d} \eta(X, Y) R \\
N^{(2)}(X, Y) & =\left(\mathcal{L}_{\phi X} \eta\right)(Y)-\left(\mathcal{L}_{\phi Y} \eta\right)(X) \\
N^{(3)}(X) & =\left(\mathcal{L}_{R} \phi\right)(X) \\
N^{(4)}(X) & =\left(\mathcal{L}_{R} \eta\right)(X) .
\end{aligned}
$$

It is clear that the tensors $N^{(i)}$ vanish for all $i=1, \ldots, 4$ if and only if the almost contact structure $(\eta, \phi, R)$ is normal. The next lemma shows that this condition is redundant.

Lemma 3.40. If $N^{(1)}$ vanishes, then $N^{(i)}=0$ for $i=2,3,4$. 
Proof. For any $X \in \mathfrak{X}(M)$ we have

$$
\begin{aligned}
0 & =N^{(1)}(X, R)=N_{\phi}(X, R)+2 \mathrm{~d} \eta(X, R) R \\
& =\phi^{2}[X, R]-\phi[\phi X, R]-R \eta(X) R-\eta([X, R]) R \\
& =-[X, R]+\eta([X, R]) R-\phi[\phi X, R]-R \eta(X) R-\eta([X, R]) R \\
& =-[X, R]-\phi[\phi X, R]-R \eta(X) R .
\end{aligned}
$$

Applying $\eta$ to 3.6 in the equation above yields $\eta([X, R])+R \eta(X)=0$. This in turns implies $\mathrm{d} \eta(R, X)=0$ for all $X \in \mathfrak{X}(M)$. Therefore $\mathcal{L}_{R} \eta=\iota_{R} \mathrm{~d} \eta=0$, i.e. $N^{(4)}=0$.

Now replacing $X$ by $\phi X$ in (3.7) in the above equation we get

$$
\begin{aligned}
N^{(1)}(\phi X, R) & =-[\phi X, R]-\phi\left[\phi^{2} X, R\right]-R \eta(\phi X) R \\
& =[R, \phi X]-\phi[-X, R]-\phi[\eta(X) R, R] \\
& =[R, \phi X]-\phi[R, X]=\left(\mathcal{L}_{R} \phi\right)(X) \\
& =N^{(3)}(X) .
\end{aligned}
$$

Thus $N^{(1)}=0$ implies $N^{(3)}=0$.

Finally, applying $\eta$ to $N^{(1)}(\phi X, Y)$ with $X, Y \in \mathfrak{X}(M)$ gives

$$
\begin{aligned}
0 & =\eta\left(N^{(1)}(\phi X, Y)\right)=\eta\left(N_{\phi}(\phi X, Y)\right)+2 \mathrm{~d} \eta(\phi X, Y) \\
& =\eta\left(\left[\phi^{2} X, \phi Y\right]\right)+2 \mathrm{~d} \eta(\phi X, Y) \\
& =-\eta([X, \phi Y])+\eta([\eta(X) R, \phi Y])+2 \mathrm{~d} \eta(\phi X, Y) \\
& =-\eta([X, \phi Y])-\phi Y \eta(X)+\eta(X) \eta([R, \phi Y])+2 \mathrm{~d} \eta(\phi X, Y) \\
& =2 \mathrm{~d} \eta(\phi X, Y)+2 \mathrm{~d} \eta(X, \phi Y)-2 \eta(X) \mathrm{d} \eta(R, \phi Y) \\
& =2\left(\left(\mathcal{L}_{\phi X} \eta\right)(Y)-\left(\mathcal{L}_{\phi Y} \eta\right)(X)\right)-2 \eta(X) \mathrm{d} \eta(R, \phi Y) \\
& =2 N^{(2)}(X, Y)-2 \eta(X) \mathrm{d} \eta(R, \phi Y)=2 N^{(2)}(X, Y) .
\end{aligned}
$$

So far we have only assumed that $(\eta, \phi, R)$ is an almost contact structure. In the case of a contact metric structure $(\eta, \phi, R, g)$ the expression of the tensors simplifies.

Proposition 3.41. The tensor fields $N^{(2)}$ and $N^{(4)}$ vanish on a contact metric manifold $(M, \eta, \phi, R, g)$. Moreover, $N^{(3)}=0$ if and only if $(\eta, \phi, R, g)$ is $K$-contact.

Proof. When $\eta$ is contact $N^{(4)}=0$ trivially. Moreover, for any $X, Y \in \mathfrak{X}(M)$ we have

$$
\begin{aligned}
N^{(2)}(X, Y) & =\left(\mathcal{L}_{\phi X} \eta\right)(Y)-\left(\mathcal{L}_{\phi Y} \eta\right)(X) \\
& =\phi X \eta(Y)-\eta([\phi X, Y])-\phi Y \eta(X)+\eta([\phi Y, X]) \\
& =2 \mathrm{~d} \eta(\phi X, Y)-2 \mathrm{~d} \eta(\phi Y, X)=2 g(Y, X)-2 g(X, Y)=0 .
\end{aligned}
$$


For the last statement recall that $\mathcal{L}_{R} \mathrm{~d} \eta=0$ because $\eta$ is contact. Now we have $\mathcal{L}_{R} g=\mathrm{d} \eta \circ\left(\mathcal{L}_{R} \phi \otimes \mathrm{Id}\right)$ because $g$ is the associated metric. Hence $R$ is Killing if $N^{(3)}$ vanishes. Conversely, if $R$ is Killing, then $N^{(3)}(X)=0$ for all $X \in \mathcal{D}$ since $\mathrm{d} \eta$ is non-degenerate on $\mathcal{D}$. Then $N^{(3)}$ vanishes identically on $M$ because $\mathcal{L}_{R} \phi(R)=0$.

\subsection{CR Structures}

Contact structures, in particular normal ones, are closely related to CR structures. In this section we recall some terminology on CR structures and explain their relation to contact structures.

Definition 3.42. An almost CR structure on a manifold $M$ is a subbundle $\mathcal{D}$ of the tangent bundle TM endowed with an almost complex structure $J$. The subbundle $(\mathcal{D}, J)$ is a CR structure if the Nijenhuis tensor $N_{J}$ vanishes and the vector field $[J X, Y]+$ $[X, J Y]$ is tangent to $\mathcal{D}$ for $X, Y \in \mathcal{D}$. In this case we will say that the almost CR structure $(\mathcal{D}, J)$ is integrable.

Equivalently, an almost CR structure can be defined as a complex subbundle $\mathcal{H}$ of the complexified tangent bundle $\mathrm{T}^{\mathbb{C}} M=\mathrm{T} M \otimes_{\mathbb{R}} \mathbb{C}$ such that $\mathcal{H} \cap \overline{\mathcal{H}}=0$. In this setting the integrability condition corresponds to $\mathcal{H}$ being closed under Lie brackets, i.e. $[X, Y]$ being tangent to $\mathcal{H}$ for any two sections $X, Y \in \mathcal{H}$. In fact, given a subbundle $\mathcal{D}$ with an almost complex structure $J$ we can define $\mathcal{H}=\{X-i J X \mid X \in \mathcal{D}\}$. Then the Lie brackets of two vectors $X, Y \in \mathcal{H}$ read

$$
[X-i J X, Y-i J Y]=[X, Y]-[J X, J Y]-i([J X, Y]+[X, J Y])
$$

so that $\mathcal{H}$ is involutive if and only if $[J X, Y]+[X, J Y]$ is tangent to $\mathcal{D}$ for $X, Y \in \mathcal{D}$ and the Nijenhuis tensor $N_{J}$ vanishes. Conversely, one can define $\mathcal{D}$ as the real part of the complex subbundle $\mathcal{H} \oplus \overline{\mathcal{H}}$ and $J$ by $J(V+\bar{V})=i(V-\bar{V})$. Also in this case is clear that the two integrability conditions are equivalent.

We focus now on the corank 1 case, that is, $M$ has dimension $2 n+1$ and $\mathcal{D}$ has rank $2 n$. In this case, assuming the orientability of $M$, there exists a 1-form $\eta$ on $M$ such that $\mathcal{D}=\operatorname{ker} \eta$ and the tangent bundle of $M$ splits non-canonically as $\mathrm{T} M=\mathcal{D} \oplus \underline{\mathbb{R}}$. Therefore, given an almost CR structure $(\mathcal{D}, J)$, we can define an endomorphism $\phi \overline{\text { of }}$ $\mathrm{T} M$ by extending $J$ trivially. This defines an almost contact structure $(\eta, \phi, R)$ where $R$ is a section of $\mathbb{R}$ such that $\eta(R)=1$. Vice versa, the distribution $\left(\mathcal{D}=\operatorname{ker} \eta, \phi_{\mid \mathcal{D}}\right)$ coming from an almost complex structure is clearly an almost CR structure.

We want to express the integrability condition of an almost CR structure in terms of the associated almost contact structure. Ianus [62] proved that normality of the almost contact structure is a sufficient condition. More precisely, this relation is given in the following:

Proposition 3.43. An almost contact structure $(\eta, \phi, R)$ on a manifold $M$ is normal if and only if 
1. the almost $C R$ structure $\left(\mathcal{D}, \phi_{\mid \mathcal{D}}\right)$ is integrable and

2. $N^{(3)}$ vanishes.

Proof. Denote by $J$ the restriction of $\phi$ to the contact distribution $\mathcal{D}$. Then we rewrite the first condition as

$$
\begin{aligned}
0 & =N_{J}(X, Y)=[J X, J Y]-[X, Y]-J([J X, Y]+[X, J Y]) \\
& =[\phi X, \phi Y]-\eta([X, Y]) R+\phi^{2}[X, Y]-\phi([\phi X, Y]+[X, \phi Y]) \\
& =N_{\phi}(X, Y)+2 \mathrm{~d} \eta(X, Y) R
\end{aligned}
$$

for $X, Y$ of $\mathcal{D}$. Moreover, we can read the second condition as

$$
0=\left(\mathcal{L}_{R} \phi\right)(X)=N^{(1)}(\phi X, R)=N_{\phi}(\phi X, R)+2 \mathrm{~d} \eta(\phi X, R) R
$$

where we used $\left(\mathcal{L}_{R} \phi\right)(X)=N^{(1)}(\phi X, R)$ from the proof of Lemma 3.40. It is clear that $N^{(1)}=0$ on $T M$ if and only if both these conditions are satisfied.

The Levi form $L$ of a corank 1 almost CR structure $(\mathcal{D}, J)$ is defined by $L(X, Y)=$ $-\mathrm{d} \eta(X, J Y)$ for $X, Y \in \mathcal{D}$, where $\eta$ is a 1 -form such that $\operatorname{ker} \eta=\mathcal{D}$. If $L$ is nondegenerate then $\eta$ is a contact form and its Reeb vector field $R$ is transverse to $\mathcal{D}$. Moreover, if the Levi form is positive or negative definite, we say that $(\mathcal{D}, J)$ is strictly pseudoconvex. In this case one can extend the Levi form to the metric $g$ associated to $\eta$ by setting $g(R, R)=1$ and $g(R, X)=0$ for $X \in \mathcal{D}$. Thus a strictly pseudoconvex almost $\mathrm{CR}$ structure defines a contact metric structure. Clearly the converse holds because a contact metric structure is in particular an almost contact structure.

\subsection{Sasaki structures}

We are now ready to introduce the main object $f$ this thesis, i.e. Sasaki manifolds. The remainder of the chapter is dedicated to Sasaki structures and their properties.

Definition 3.44. A Sasaki structure $(\eta, \phi, R, g)$ is a contact metric structure whose underlying almost contact structure is normal, i.e. such that $N^{(1)}=0$. A Sasaki manifold $(M, \eta, \phi, R, g)$ is a smooth manifold $M$ equipped with a Sasaki structure $(\eta, \phi, R, g)$. A manifold $M$ admitting a Sasaki structure is called a Sasakian manifold.

Notation. In order to lighten the notation we may write M for a Sasaki manifold. In this case the Sasaki structure is understood to be fixed.

As in the K-contact case several different structures with suitable compatibility conditions come together in the definition of Sasaki structures. The abundance of underlying structures allows many approaches to Sasakian geometry. In analogy with the $\mathrm{K}$-contact case we discuss now various definitions of Sasaki manifolds. 
We begin by investigating the relation between Sasaki and K-contact structures. It turns out that every Sasaki structure has a canonical underlying K-contact structure.

Corollary 3.45. A Sasaki structure $(\eta, \phi, R, g)$ is $K$-contact.

Proof. This is a direct consequence of Lemma 3.40 and Proposition 3.41 .

Remark 3.46. Corollary 3.45 implies that Sasakian manifolds satisfy the topological properties of K-contact manifolds presented in Section 3.3, in particular tori are not Sasakian.

It is then natural to ask which additional conditions guarantee that a K-contact manifold is indeed Sasaki. Combining Proposition 3.43 with Proposition 3.41 we get a necessary and sufficient condition for a K-contact structure to be Sasaki. Namely, a Sasaki structure consists of a K-contact structure satisfying an integrability condition.

Corollary 3.47. A $K$-contact structure $(\eta, \phi, R, g)$ is Sasaki if and only if the underlying almost $C R$ structure is integrable.

This leads to two different viewpoints on Sasakian geometry. One may think of Sasaki structures as strictly pseudoconvex CR structures $(\mathcal{D}, J)$ whose associated Reeb vector field preserves $J$. In fact, such a CR structure defines a contact structure. The Levi form $L$ defines a transverse metric compatible with the contact structure. Since $J$ is $R$-invariant this defines a $\mathrm{K}$-contact structure, that is, a Sasaki structure because we assumed the CR structure to be integrable.

Sasaki structures can also be regarded as K-contact structures whose underlying CR structure is integrable. Hence, bearing in mind the additional integrability condition, the discussion that led to Definition 3.30 applies to Sasaki structures. In particular, one can view Sasaki structures as contact forms with the choice of an integrable compatible almost complex structure $J$ which is $R$-invariant.

Sasaki manifolds can also be characterized as contact metric manifold whose metric cone is Kähler.

Proposition 3.48. A contact metric manifold $(M, \eta, \phi, R, g)$ is Sasaki if and only if the cone $\left(M \times \mathbb{R}^{+}, d\left(t^{2} \eta\right), I\right)$ is Kähler.

Proof. It is clear that the form $\mathrm{d}\left(t^{2} \eta\right)$ is symplectic and the almost complex structure $I$ is compatible with it. By definition a metric contact structure is normal if the associated almost complex structure on the metric cone is integrable. The claim then follows directly from Definition 3.44 .

In fact, the analogy between Sasaki and Kähler manifolds goes further than Proposition 3.48. Namely, we can characterize Sasaki structures as contact forms with a specific transverse Kähler geometry. In Section 3.3 we have discussed the analogy between K-contact structures and almost Kähler structures. Specifically, we have seen that a $\mathrm{K}$-contact manifold $(M, \eta, \phi, R, g)$ is a contact manifold $(M, \eta)$ with an $R$-invariant 
transverse almost Kähler structure of the form $\left(\mathrm{d} \eta, J, g_{\mathcal{D}}\right)$. Since a Sasaki manifold is $\mathrm{K}$-contact, the same applies in this context but more can be said. In fact, the transverse almost complex structure $J$ is integrable. That is, there exist foliated charts for the characteristic foliation such that the transverse transition functions are holomorphic. This is equivalent to integrability of the underlying almost CR structure.

Summarizing the discussion above we get several equivalent definitions of Sasaki structures:

Definition 3.49. A Sasaki structure on a smooth manifold $M$ is given by one of the following equivalent structures:

i) A contact metric structure $(\eta, \phi, R, g)$ whose underlying almost contact structure is normal.

ii) A contact metric structure $(\eta, \phi, R, g)$ whose metric cone $\left(M \times \mathbb{R}^{+}, \mathrm{d}\left(t^{2} \eta\right), I\right)$ is Kähler.

iii) A strictly pseudoconvex $\mathrm{CR}$ structure $(\mathcal{D}, J)$ such that the associated Reeb vector field $R$ preserves $J$, i.e. $\mathcal{L}_{R} J=0$.

iv) A strictly pseudoconvex $\mathrm{CR}$ structure $(\mathcal{D}, J)$ such that the associated Reeb vector field $R$ preserves the Levi form $L$, i.e. $\mathcal{L}_{R} L=0$.

v) A contact form $\eta$ with an integrable compatible almost complex structure $J$ such that the flow of $R$ preserves $J$, i.e. $\mathcal{L}_{R} J=0$.

vi) A contact form $\eta$ with a metric $g_{\mathcal{D}}$ associated to a complex structure $J$ such that the flow of $R$ preserves $g_{\mathcal{D}}$, i.e. $\mathcal{L}_{R} g_{\mathcal{D}}=0$.

vii) A contact form $\eta$ with a transverse Kähler structure of the form $\left(\mathrm{d} \eta, J, g_{\mathcal{D}}\right)$.

viii) A K-contact structure $(\eta, \phi, R, g)$ whose underlying almost $\mathrm{CR}$ structure is integrable.

These equivalences justify the heuristic of regarding Sasaki manifolds as the odd dimensional analogues of Kähler manifolds. Moreover, it is clear that Sasaki manifolds relate to K-contact manifolds as Kähler manifolds relate to almost Kähler manifolds. In fact, a Sasaki structure, respectively Kähler structure, is given by a K-contact strcture, resp. almost Kähler triple, whose almost complex structure is integrable. We refer to the discussion following Theorem 3.59 for further details.

Note that a Sasaki structure $(\eta, \phi, R, g)$ is uniquely determined by the underlying CR structure $\left(\operatorname{ker} \eta=\mathcal{D}, \phi_{\left.\right|_{\mathcal{D}}}=J\right)$ and the Reeb vector field $R$. In order to see this suppose $(\mathcal{D}, J)$ and $R$ are given. Then the endomorphism $\phi$ is determined by simply extending $J$ trivially to $T M$. Now, since $\mathcal{D}$ is a contact distribution, there exists a contact form $\eta^{\prime}$ such that $\operatorname{ker} \eta^{\prime}=\mathcal{D}$. Therefore we can recover $\eta$ by setting $\eta=\frac{\eta^{\prime}}{f}$ where $f=\eta^{\prime}(R)$. We want to show that $R$ is indeed the Reeb vector field for $\eta$. Notice that $\mathcal{L}_{R} \eta=f^{\prime} \eta$ since 
$R$ preserves the contact distribution. Moreover, $\mathcal{L}_{R} \eta=\iota_{R} \mathrm{~d} \eta+\mathrm{d} \iota_{R} \eta=\iota_{R} \mathrm{~d} \eta$. Therefore we get

$$
f^{\prime}=f^{\prime} \eta(R)=\mathcal{L}_{R} \eta(R)=\iota_{R} \mathrm{~d} \eta(R)=0 .
$$

We conclude that $R$ is the Reeb vector field of $\eta$. Now the Sasaki structure is determined by the identity $g=\mathrm{d} \eta \circ \mathrm{Id} \otimes \phi+\eta \otimes \eta$.

The above discussion suggests that it may be fruitful to consider deformations of Sasaki structures that leave the CR structure or the Reeb vector field invariant. A deformation of the former type is called a deformation of type $\mathrm{I}$.

Definition 3.50. Let $(M, \eta, \phi, R, g)$ be a Sasaki manifold and denote by $(\mathcal{D}, J)$ the underlying strictly pseudoconvex CR structure. Let $\mathcal{S}(\mathcal{D}, J)$ be the space of Sasaki structures with underlying $\mathrm{CR}$ structure $(\mathcal{D}, J)$. A deformation of type $\mathbf{I}$ is a deformation of the Sasaki structure that leaves the underlying CR structure invariant, i.e. a deformation inside $\mathcal{S}(\mathcal{D}, J)$.

Deformations of type I have a very explicit description. Namely, let $\left(\eta_{t}, \phi_{t}, R_{t}, g_{t}\right)$ be such a deformation. Since the contact distribution is preserved we have $\eta_{t}=f_{t} \eta$ and $R_{t}=R+\rho_{t}$ where $f_{t}$ is a non-vanishing function and $\rho$ is a vector field. Moreover, $f_{t}$ has the form

$$
f_{t}=\frac{1}{\eta\left(R_{t}\right)}=\frac{1}{1+\eta\left(\rho_{t}\right)}
$$

because $\eta_{t}\left(R_{t}\right)=1$. This implies $\eta\left(\rho_{t}\right)>-1$. The endomorphism $\phi_{t}$ is then defined by $\phi_{t}=\phi-\phi R_{t} \otimes \eta_{t}$ and the metric $g_{t}$ is determined by

$$
g_{t}=\mathrm{d} \eta_{t} \circ \mathrm{Id} \otimes \phi_{t}+\eta_{t} \otimes \eta_{t} .
$$

Conversely a deformation of the form $R \mapsto R+\rho_{t}$ with $\eta\left(\rho_{t}\right)>-1$ yields a deformation of type I.

Now given a Sasaki manifold $(M, \eta, \phi, R, g)$ we can consider deformations that leave the Reeb vector field unchanged. Such deformations are given by a family of structures $\left(\eta_{s}=\eta+\zeta_{s}, \phi_{s}, R, g_{s}\right)$ with the following properties. The form $\zeta_{s}$ is a basic 1 -form, i.e. $\mathcal{L}_{R} \zeta_{s}=\zeta_{s}(R)=0$, and $\eta_{s} \wedge\left(\mathrm{d} \eta_{s}\right)^{n}$ is nowhere vanishing. It is clear that $R$ is the Reeb vector field of $\eta_{s}$. The endomorphism $\phi_{s}$ is then given by

$$
\phi_{s}=\phi-R \otimes \zeta_{s} \circ \phi
$$

and the metric $g_{s}$ is associated to $\eta_{s}$ and $\phi_{s}$ by by

$$
g_{s}=\mathrm{d} \eta_{s} \circ \mathrm{Id} \otimes \phi_{s}+\eta_{s} \otimes \eta_{s} .
$$

We focus now on deformations that preserve the Reeb foliation $\mathcal{F}$ but not necessarily the Reeb vector field $R$. One can obtain such a deformation by composing a deformation that fixes the Reeb vector field $R$ with a rescaling of $R$ by a constant function, i.e. $R \mapsto$ $a R$ for $a \in \mathbb{R} \backslash\{0\}$. 
Definition 3.51. Let $(M, \eta, \phi, R, g)$ be a Sasaki manifold and denote by $\mathcal{F}$ the Reeb foliation. Let $\mathcal{S}(\mathcal{F})$ be the space of Sasaki structures with Reeb foliation $\mathcal{F}$. A deformation of type II is a deformation of the Sasaki structure that leaves the Reeb foliation invariant, i.e. a deformation inside $\mathcal{S}(\mathcal{F})$.

Later in this thesis we will also study Sasaki structures up to isomorphisms and equivalences.

Definition 3.52. Let $\left(M_{1}, \eta_{1}, \phi_{1}, R_{1}, g_{1}\right)$ and $\left(M_{2}, \eta_{2}, \phi_{2}, R_{2}, g_{2}\right)$ be two Sasaki manifolds. A diffeomorphism $f: M_{1} \longrightarrow M_{2}$ is an isomorphism of Sasaki manifolds if

$$
f^{*} \eta_{2}=\eta_{1} \text {, and } f_{*} \phi_{1}=\phi_{2} f_{*}
$$

(hence clearly $f^{*} g_{2}=g_{1}$ and $f_{*} R_{1}=R_{2}$ ). The two Sasaki structures $\left(\eta_{1}, \phi_{1}, R_{1}, g_{1}\right)$ and $\left(\eta_{2}, \phi_{2}, R_{2}, g_{2}\right)$ are then called isomorphic.

Definition 3.53. Two Sasaki structures $\left(\eta_{1}, \phi_{1}, R_{1}, g_{1}\right)$ and $\left(\eta_{2}, \phi_{2}, R_{2}, g_{2}\right)$ on a manifold $M$ are called equivalent if they can by identified by a sequence of isomorphisms and smooth deformations. In this case the smooth deformations are arbitrary, hence, in particular, they need not to be of type I and II.

We present now the structure theorem for Sasaki and K-contact manifolds which will play a key role in this thesis. In order to do so we recall some classical results. The first of these results is a theorem of Wadsley which gives a necessary and sufficient condition for foliations by circles to be induced by smooth $S^{1}$-actions.

Theorem 3.54 ([115]). Let $M$ be a manifold and $\mathcal{F}$ a foliation of $M$ by circles. The leaves of $\mathcal{F}$ are the orbits of a smooth $S^{1}$-action if and only if there exists a metric $g$ on $M$ for which the leaves of $\mathcal{F}$ are geodesics.

Remark 3.55. The condition in Theorem 3.54 is always satisfied on K-contact manifolds or, more generally, contact metric manifolds. In fact, since the metric $g$ is compatible with $\eta$ (cf. Definition 3.4), the equality $\eta(X)=g(X, R)$ holds. Therefore we get

$$
\begin{aligned}
0=\mathrm{d} \eta(R, X) & =R(\eta(X))-X(\eta(R))-\eta([R, X]) \\
& =R(g(R, X))-\eta\left(\nabla_{R} X\right)+\eta\left(\nabla_{X} R\right) \\
& =g\left(\nabla_{R} R, X\right)+g\left(R, \nabla_{R} X\right)-g\left(R, \nabla_{R} X\right)+\frac{1}{2} X(g(R, R)) \\
& =g\left(\nabla_{R} R, X\right)
\end{aligned}
$$

for all $X \in \mathfrak{X}(M)$. This shows that the orbits of $R$ are geodesics for $g$.

Corollary 3.56. Let $(M, \eta, \phi, R, g)$ be a $K$-contact manifold such that the orbits of $R$ are circles. Then the flow of $R$ induces a locally free smooth $S^{1}$-action. 
Proof. By Remark 3.55, the leaves of the Reeb foliation are geodesics for the metric $g$. Therefore, Theorem 3.54 implies that the flow of $R$ defines a smooth $S^{1}$-action. Moreover, since $R$ is nowhere vanishing, the action is locally free.

We consider now compact K-contact manifolds. In this case the following theorem of Rukimbira implies that if a compact manifold admits a K-contact structure, then it admits one whose leaves of the Reeb fibration are circles, see [101]. We will state and prove the result for $\mathrm{K}$-contact manifolds even though it was originally proved in a slightly different, although equivalent, setting.

Theorem 3.57 ([101]). Let $(M, \eta, \phi, R, g)$ be a compact $K$-contact (Sasaki) manifold. Then $M$ admits a quasi-regular $K$-contact (Sasaki) structure.

Proof. Let $(M, \eta, \phi, R, g)$ be a compact K-contact manifold. The Reeb vector field $R$ is Killing and, by Proposition 3.41, it preserves the endomorphism $\phi$. Therefore, its flow defines a homomorphism

$$
\varphi: \mathbb{R} \longrightarrow \operatorname{Isom}(M, g) \cap \operatorname{CR}(M, \mathcal{D})
$$

where $\operatorname{CR}(M, \mathcal{D})$ is the group of CR-transformations of $(M, \mathcal{D})$. If the structure is irregular, then the image of this homomorphism is not $S^{1}$. Nevertheless, its closure is an abelian subgroup, hence a torus because $\operatorname{Isom}(M, g)$ is a compact Lie group. We can now pick an $S^{1}$ in this torus corresponding to a vector field $R^{\prime}$ arbitrarily close to $R$. Consider the form

$$
\eta^{\prime}=\frac{\eta}{\eta\left(R^{\prime}\right)}
$$

This is clearly a contact form because $R^{\prime}$ is close enough to $R$ and its Reeb vector field is $R^{\prime}$. With respect to the splitting $T M=\mathcal{D} \oplus L_{R}$ the metric $g$ is given by $g=g_{\mathcal{D}} \oplus \eta \otimes \eta$ where $\mathcal{D}=\operatorname{ker} \eta=\operatorname{ker} \eta^{\prime}$ and $g_{\mathcal{D}}=\mathrm{d} \eta \circ(\phi \otimes \mathrm{Id})$. Let $\phi^{\prime}$ be an endomorphism of $T M$ defined by

$$
\phi_{\mid \mathcal{D}}^{\prime}=\phi_{\mid \mathcal{D}}, \quad \phi^{\prime}\left(R^{\prime}\right)=0 .
$$

We define a metric $g^{\prime}$ on $M$ as

$$
g^{\prime}=\mathrm{d} \eta^{\prime} \circ\left(\phi^{\prime} \otimes \mathrm{Id}\right)+\eta^{\prime} \otimes \eta^{\prime} .
$$

Since $R^{\prime}$ is the Reeb vector field of $\eta^{\prime}$, its flow preserves $\eta^{\prime}$ and $\mathrm{d} \eta^{\prime}$. Moreover, it preserves $\phi$ because it lies in the closure of the image of the homomorphism $\varphi$. We conclude that $R^{\prime}$ is Killing for $g^{\prime}$ and it preserves the CR structure. Therefore $\left(\eta^{\prime}, \phi^{\prime}, R^{\prime}, g^{\prime}\right)$ is a quasi-regular K-contact structure on $M$.

By Corollary 3.47, the K-contact structure $(\eta, \phi, R, g)$ is Sasaki if and only if the underlying almost $\mathrm{CR}$ structure $\left(\mathcal{D}, \phi_{\mid \mathcal{D}}\right)$ is integrable. Notice that the underlying almost CR structure did not change in deforming $(\eta, \phi, R, g)$ into $\left(\eta^{\prime}, \phi^{\prime}, R^{\prime}, g^{\prime}\right)$ because, by definition, $\phi_{\mid \mathcal{D}}^{\prime}=\phi_{\mid \mathcal{D}}$ and $\operatorname{ker} \eta=\operatorname{ker} \eta^{\prime}$. Therefore, $\left(\eta^{\prime}, \phi^{\prime}, R^{\prime}, g^{\prime}\right)$ is Sasaki if and only if $(\eta, \phi, R, g)$ is Sasaki. 
Remark 3.58. By compactness, the leaves of a quasi-regular K-contact (Sasaki) structure on a compact manifold are circles. Thus Theorem 3.57 implies that a compact (possibly irregular) K-contact or Sasaki manifold admits a locally free $S^{1}$-action given by the flow of the Reeb vector field of a quasi-regular structure.

We are now ready to prove the structure theorem for K-contact and Sasaki manifolds. We state this theorem in a compact form that collects several results. Namely, Theorem 6.3.8, Theorem 7.1.3 and Theorem 7.1.6 in [15].

Theorem 3.59 (Structure Theorem [15]). Let $(M, \eta, \phi, R, g)$ be a compact quasi-regular $K$-contact manifold and $\pi: M \longrightarrow X$ the projection on the space of orbits of $R$. Then

i) $X$ admits a symplectic cyclic orbifold structure $\mathcal{X}=(X, \mathcal{U})$ with symplectic form $\omega$.

ii) $\pi: M \rightarrow \mathcal{X}$ is a principal $S^{1}$-orbibundle with connection 1-form $\eta$ and curvature $d \eta=\pi^{*} \omega$.

iii) $(M, \eta, \phi, R, g)$ is Sasaki if and only if $\omega$ is a Kähler form on $\mathcal{X}$.

iv) The orbifold structure on $\mathcal{X}$ is trivial if and only if the K-contact (Sasaki) structure is regular.

Conversely, let $\mathcal{X}=(X, \mathcal{U})$ be an almost Kähler orbifold with integral symplectic class $[\omega]$. Then the principal $S^{1}$-orbibundle $M \stackrel{\pi}{\rightarrow} X$ associated to $[\omega]$ is a quasi-regular $K$-contact orbifold with contact form $\eta$ such that $d \eta=\pi^{*} \omega$. Moreover, M is Sasaki if and only if $\mathcal{X}$ is a Kähler orbifold.

Proof. We refer to Chapter 2 for the theory of orbifolds. By quasi-regularity and compactness, every orbit can be covered by finitely many foliated charts and the intersection of the orbit with each chart has finitely many connected components. Therefore, all orbits are circles. By Corollary 3.56, the flow of the Reeb vector field defines a locally free $S^{1}$-action.

Now the first two claims follow from the Slice Theorem for smooth actions. Namely, let $x \in X$ be an orbit and $p$ a point on the orbit $x$. Let $\mathbb{Z}_{n}$ be the isotropy group of $p$. There exists a tubular neighbourhood of the form $S_{x} \times_{\mathbb{Z}_{n}} S^{1} \subset M$ where $S_{x}$ is diffeomorphic to (a neighbourhood of the zero section of) the normal bundle, i.e. the contact distribution $\mathcal{D}$. Let $\Gamma_{x} \subset \mathbb{Z}_{n}$ be the subgroup of the isotropy group acting effectively on the slice $S_{x}$. Thus we can define an orbifold atlas $\mathcal{U}$ on $X$ consisting of charts of the form $\left(S_{x}, \Gamma_{x}, \pi_{\mid S_{x}}\right)$ around $x \in X$. Notice that iv) follows immediately from this description.

Now $\pi: M \rightarrow \mathcal{X}$ is a principal $S^{1}$-orbibundle with charts $\left(S_{x} \times_{\mathbb{Z}_{n}} S^{1}, \Gamma_{x}, \pi\right)$ since the $S^{1}$-action is locally free. It is clear that $\mathcal{D}$ defines an Ehresmann connection for the $S^{1}$-bundle with connection 1-form $\eta$. Let $\widetilde{V}$ denote the horizontal lift of a vector field $V$ on $\mathcal{X}$. Then the formula $J V=\pi_{*} \phi \widetilde{V}$ gives a well defined almost complex structure $J$ on $\mathcal{X}$ because $\phi$ is invariant under the flow of $R$. Similarly, $\omega(V, W)=\mathrm{d} \eta(\widetilde{V}, \widetilde{W})$ defines 
a symplectic form on $\mathcal{X}$ compatible with $J$. Therefore, $(\mathcal{X}, \omega, J)$ is an almost Kähler orbifold and we have proven claims $i$ ), ii) and $i v$ ).

Finally $(\mathcal{X}, \omega, J)$ is Kähler if and only if $J$ is integrable. This holds if and only if the underlying almost CR structure is integrable because $N_{J}[V, W]=\pi_{*} N_{\phi_{\mid \mathcal{D}}}[\widetilde{V}, \widetilde{W}]$. Thus iii) follows from Corollary 3.47 .

Conversely, let $\mathcal{U}=\left\{\left(\widetilde{U}_{i}, \Gamma_{i}, \varphi_{i}\right\}\right.$ and denote embeddings by $\lambda_{i j}: \widetilde{U}_{i} \rightarrow U_{j}$. The integrality of $[\omega]$ means that there exists a class $e \in H_{\text {orb }}^{2}(\mathcal{X} ; \mathbb{Z})$ mapping to $[\omega]$ under the inclusion $H_{\text {orb }}^{2}(\mathcal{X} ; \mathbb{Z}) \hookrightarrow H_{o r b}^{2}(\mathcal{X} ; \mathbb{R})$. By Proposition 2.34 , the class $[\omega]$ determines a principal $S^{1}$-orbibundle $M \stackrel{\pi}{\rightarrow} \mathcal{X}$ with first Chern class $c_{1}^{o r b}(M)=e$. Namely, it determines the transition maps $\lambda_{i j}^{*}$ and the homorphisms $h_{\widetilde{U}_{i}}: \Gamma_{i} \rightarrow G$ in Definition 2.6 Moreover, there is an atlas of $M$ given by charts of the form $\left(E_{i}, \Gamma_{i}^{*}, \varphi_{i}^{*}\right)$ where $E_{i}=$ $\tilde{U}_{i} \times S^{1}$.

The form $\omega$ is given by gluing together a collection of invariant forms $\omega_{i} \in \Omega^{2}\left(\widetilde{U}_{i}\right)$. By the argument given in Section 3.2 there exist a connection 1-form $\eta$ on $M$ such that $\mathrm{d} \eta=\pi^{*} \omega$. This amounts to the existence of connections $\eta_{i}$ for the $S^{1}$-bundle $E_{i}$ such that $\mathrm{d} \eta_{i}=\pi_{E_{i}}^{*} \omega_{i}$ satisfying the compatibility conditions. Now let $R_{i}$ be the vector field in $\mathrm{T} S^{1} \subset \mathrm{T} E_{i}$ such that $\eta_{i}\left(R_{i}\right)=1$. Clearly we have $\mathrm{d} \eta_{i}\left(R_{i}\right)=0$. Consider a basis $\left(V_{1}, \ldots, V_{2 n}, R_{i}\right)$ for $\mathrm{T} E_{i}$ with $V_{1}, \ldots, V_{2 n} \in \operatorname{ker} \eta_{i}$. Then

$$
\begin{aligned}
& \eta_{i} \wedge\left(\mathrm{d} \eta_{i}\right)^{n}\left(V_{1}, \ldots, V_{2 n}, R_{i}\right)=\eta_{i}\left(R_{i}\right)\left(\mathrm{d} \eta_{i}\right)^{n}\left(V_{1}, \ldots, V_{2 n}\right) \\
& =\left(\pi^{*} \omega\right)^{n}\left(V_{1}, \ldots, V_{2 n}\right)=\omega^{n}\left(\pi_{*} V_{1}, \ldots, \pi_{*} V_{2 n}\right) \neq 0
\end{aligned}
$$

because $\pi_{*}$ is an isomorphism when restricted to $\operatorname{ker} \eta_{i}$. Therefore, $\eta$ is a contact form on $M$ because $\eta_{i} \wedge\left(\mathrm{d} \eta_{i}\right)^{n}$ is non-vanishing. Moreover, the $R_{i}$ 's patch together to give the Reeb vector field $R$ of $\eta$.

Let $J$ be the almost complex structure on $X$ and $h$ the associated metric. Once again we lift $h$ to a metric on the subbundle $\mathcal{D}=\operatorname{ker} \eta \subset \mathrm{T} M$. Define then a metric $g$ on $M$ by setting $g=\pi^{*} h+\eta \otimes \eta$. Moreover, for $V \in \mathrm{T} M$ we can define $\phi V=\widetilde{J \pi_{*}(V)}$ where $\widetilde{X}$ denotes the horizontal lift of $X \in \mathrm{T} \widetilde{U}_{i}$. Then the discussion in Section 3.3 shows that $(M, \eta, \phi, R, g)$ is a K-contact manifold by construction.

Moreover, since $\pi_{*}$ is an isomorphism on $\mathcal{D}$, the underlying almost CR structure is integrable if and only if the almost complex structure $J$ is integrable. In that case $\mathcal{X}$ is a Kähler orbifold.

The Structure Theorem 3.59 further justifies the analogy between Kähler and Sasakian geometry. Moreover, for quasi-regular structures it is even more evident that Sasaki manifolds relate to K-contact manifolds as Kähler manifolds relate to almost Kähler manifolds. Namely, a quasi-regular K-contact manifold $M$ is a principle $S^{1}$ orbibundle over a symplectic orbifold $(\mathcal{X}, \omega)$. The K-contact structure $(\eta, \phi, R, g)$ on $M$ determines an almost Kähler triple $(\omega, J, h)$ on $\mathcal{X}$ and vice versa. The almost Kähler structure is then Kähler if and only if the K-contact structure is Sasaki.

Remark 3.60. We have already seen that an orbibundle has a trivial orbifold structure 
if the uniformizing groups of the base inject into the structure group. Therefore the orbibundle $M$ in Theorem 3.59 can be manifold only if the symplectic orbifold $\mathcal{X}$ is cyclic, i.e. all its uniformizing groups are cyclic.

Remark 3.61. The form $\omega$ represents the Euler class of the bundle $M \rightarrow \mathcal{X}$. Hence it defines an integral class. In the Sasakian case this implies that the base orbifold is a projective variety, see Theorem 2.54. Moreover, the topological space $X$ underlying the orbifold $X$ is the quotient of a smooth manifold by a locally free smooth $S^{1}$-action. Thus the space $X$ is a normal variety with cyclic quotient singularities and falls into the discussion of Section 2.4. In this sense Sasakian geometry can be considered the odd dimensional counterpart to projective geometry rather than Kähler geometry.

Remark 3.62. By Theorem 3.57, any compact K-contact (Sasaki) manifold $M$ admits a quasi-regular structure. Therefore, $M$ is always the total space of a principal $S^{1}$ orbibundle over a symplectic (projective) orbifold. Moreover, by Theorem 3.22, $M$ is a Boothby-Wang bundle over a symplectic (projective) manifold if and only if it admits a regular K-contact (Sasaki) structure.

Remark 3.63. While every Sasakian manifold admits a quasi-regular structure, it is still an open question whether all Sasakian manifolds admit a regular structure. In other words, it is not known whether all Sasakian manifolds arise as Boothby-Wang bundles over projective manifolds.

We conclude this section by presenting some examples of Sasaki structures. We begin with the odd dimensional sphere $S^{2 n+1}$. In Example 3.24 we have seen the standard contact structure on $S^{2 n+1}$, cf. Example 3.19, as a Boothby-Wang bundle over the complex projective space $\mathbb{C P}^{n}$ equipped with the Fubini-Study form. The Structure Theorem 3.59 implies that the standard contact structure on $S^{2 n+1}$ is indeed a regular Sasaki structure. In fact, every projective manifold $X$ with integral Kähler class $[\omega]$ provides an example of regular Sasaki structure, see for instance Example 4.19 and Example 4.20. Next we present some irregular and quasi-regular Sasaki structures on $S^{2 n+1}$.

Example 3.64. We produce now irregular contact structures on $S^{2 n+1}$ as type I deformations of the standard Sasaki structure $\left(\eta_{0}, \phi_{0}, R_{0}, g_{0}\right)$, see the description after Definition 3.50. As usual we regard the sphere $S^{2 n+1}$ as the space of unit vectors in $\mathbb{C}^{n+1}$. Define

$$
R_{w}=\sum_{i=0}^{n} w_{i}\left(y_{i} \frac{\partial}{\partial x_{i}}-x_{i} \frac{\partial}{\partial y_{i}}\right)
$$

where $w=\left(w_{0}, \ldots, w_{n}\right)$ and $w_{i}$ is a positive real number for each $i=0, \ldots, n$. The vector field $R_{w}$ is the Reeb vector field for the contact form

$$
\eta_{w}=\frac{\eta_{0}}{\sum_{i=0}^{n} w_{i}\left(x_{i}^{2}+y_{i}^{2}\right)} .
$$

Following the description of deformations of type I we can write $R_{w}=R_{0}+\rho_{w}$. Notice 
that

$$
\eta\left(\rho_{w}\right)=\eta\left(R_{w}\right)-1=\sum_{i=0}^{n} w_{i}\left(x_{i}^{2}+y_{i}^{2}\right)-1>-1
$$

so that $R_{w}$ defines indeed a type I deformation $\left(\eta_{w}, \phi_{w}, R_{w}, g_{w}\right)$ of the standard structure where $\phi_{w}=\phi-\phi R_{w} \otimes \eta_{w}$ and $g_{w}$ is determined by

$$
g_{w}=\mathrm{d} \eta_{w} \circ \mathrm{Id} \otimes \phi_{w}+\eta_{w} \otimes \eta_{w} .
$$

We denote by $S^{2 n+1}(w)$ the sphere $S^{2 n+1}$ as a Sasaki manifold endowed with the Sasaki structure $\left(\eta_{w}, \phi_{w}, R_{w}, g_{w}\right)$. It is easy to see that the structure just defined is irregular unless $w_{i} \in \mathbb{Q}$ for all $i=0, \ldots, n$. This instance is described in further detail in Example 3.65 below.

Example 3.65. Consider the weighted sphere $S^{2 n+1}(w)$ defined in Example 3.64. Assume that $w_{0} \leqslant \cdots \leqslant w_{n}$ and that $w_{i} \in \mathbb{Q}$ for all $i=0, \ldots, n$. Then the orbits of $R_{w}$ are circles, i.e. the structure $\left(\eta_{w}, \phi_{w}, R_{w}, g_{w}\right)$ is quasi-regular.

By the Structure Theorem 3.59 this determines a $S^{1}$-orbibundle $\pi: S^{2 n+1}(w) \longrightarrow \mathcal{X}$ over a projective orbifold $(\mathcal{X}, \omega)$. Without loss of generality we can multiply $w$ by the $\mathrm{lcm}$ of the denominators and redefine the action to get $0<w_{i} \in \mathbb{Z}$. Moreover we can divide by the gcd of the integers so obtained to achieve the condition $\operatorname{gcd}\left(w_{0}, \ldots, w_{n}\right)=$ 1. This does not change the space of leaves of the $S^{1}$-action, i.e. the base orbifold $\mathcal{X}$, but it rescales the Kähler form $\omega$ on $\mathcal{X}$.

Recall the $\mathbb{C}^{*}(w)$ action on $\mathbb{C}^{n+1}$ defined in Example 2.37. Now the orbits of $R_{w}$ coincide with the restriction to $S^{2 n+1}$ of the orbits of the $\mathbb{C}^{*}(w)$-action. Thus the base orbifold $\mathcal{X}$ of the fibration is the weighted projective space $\mathbb{C P}^{n}(w)$ defined in Example 2.37. Namely, there exists a unique Kähler form $\omega$ on $\mathbb{C P}^{n}(w)$ such that $\pi^{*}(\omega)=\eta_{w}$. The class $[\omega]$ is the image in $H_{\text {orb }}^{2}\left(\mathbb{C P}^{n}(w) ; \mathbb{R}\right)$ of the first Chern class of the principal orbibundle $\pi: S^{2 n+1}(w) \longrightarrow \mathbb{C P}^{n}(w)$.

Example 3.66 (Links). Consider the $\mathbb{C}^{*}(w)$ action on $\mathbb{C}^{n+1}$ of Example 2.37 and let $f$ be a weighted homogeneous polynomial as in Definition 2.43. Recall that $f$ defines a hypersurface in $\mathbb{C P}^{n}(w)$ if $\operatorname{pr}^{-1}\left(X_{f}\right) \subset \mathbb{C}^{n+1} \backslash\{0\}$ is smooth, see Proposition 2.45 . Notice that this condition is equivalent to smoothness of the set of zeroes $V_{f}$ of $f$. We will assume that $f$ satisfies this condition. The link of $V_{f}$ is the smooth manifold $L_{f}$ given by the intersection

$$
L_{f}=V_{f} \bigcap S^{2 n+1} .
$$

If $0 \in \mathbb{C}^{n+1}$ is a regular point for $f$, then $L_{f}$ is diffeomorphic to the standard sphere $S^{2 n-1}$ by the Morse Lemma, see [87, Lemma 2.12]. Hence we will assume that $0 \in \mathbb{C}^{n+1}$ is a singular point for $f$, that is, $f$ has no linear terms. As a differentiable manifold $L_{f}$ is rather simple. Namely, it is $(n-2)$-connected by Milnor's Fibration Theorem [87].

We want to show that $L_{f}$ supports a Sasaki structure. More precisely, the structure $\left(\eta_{w}, \phi_{w}, R_{w}, g_{w}\right)$ on $S^{2 n+1}(w)$ of Example 3.64 induces a Sasaki structure on $L_{f}$. In order to show that we need the following: 
Definition 3.67. Let $(M, \eta, \phi, R, g)$ be a Sasaki manifold. An immersed submanifold $N$ is said to be an invariant submanifold if

1. $R$ is tangent to $T N$ at all points $p$ of $N$ and

2. $\phi T_{p} N \subset T_{p} N$ for all $p \in N$.

The following result shows that an invariant submanifold is a Sasaki manifold in a natural way.

Lemma 3.68 ([96]). An invariant submanifold $N$ of a Sasaki manifold $(M, \eta, \phi, R, g)$ is Sasaki with the structure given by the restriction of $(\eta, \phi, R, g)$ to $N$.

Proof. Clearly the restrictions of $\eta$ and $g$ to $N$ are well defined. The two conditions in Definition 3.67 ensure that $R$ and $\phi$ are well defined on $N$. Moreover they satisfy on $N$ all the compatibility conditions that are satisfied on $M$. Thus $(N, \eta, \phi, R, g)$ is a Sasaki manifold.

We can now show that $L_{f}$ inherits a Sasaki structure from $S^{2 n+1}(w)$. According to Lemma 3.68 we have to show that

1. $R_{w}$ is everywhere tangent to $T L_{f}$ and

2. $\phi_{w} T_{p} L_{f} \subset T_{p} L_{f}$ for all $p \in L_{f}$.

Let $\lambda$ be an element of $S^{1} \subset \mathbb{C}^{*}$. Differentiating the equation $f(\lambda z)=\lambda^{d} f(z)$ in the direction of $R_{w}$ we get

$$
R_{w}(f)=\mathrm{d} f\left(R_{w}\right) d \cdot f .
$$

Thus $\mathrm{d} f\left(R_{w}\right)$ vanishes along $V_{f}$ and, a fortiori, along $L_{f}$. In order to prove the second claim denote by $J$ the standard complex structure of $\mathbb{C}^{n+1}$. The endomorphism $\phi_{w}$ coincides with $J$ both on $\mathcal{D}_{w}=\operatorname{ker} \eta_{w}$ and on $\mathcal{D}_{L_{f}}=\operatorname{ker} \eta_{L_{f}}$, where we are writing $\eta_{L_{f}}$ for $\eta_{w}$ restricted to $L_{f}$ to avoid the clash of notation. Since $\phi_{w} R_{w}=0$ condition 2 in Definition 3.67 becomes $\phi_{w} \mathcal{D}_{L_{f}} \subset \mathcal{D}_{L_{f}}$. Notice that $\mathcal{D}_{L_{f}}=\mathcal{D}_{w} \bigcap T V_{f}$. Now $\phi_{w}$ leaves $\mathcal{D}_{w}$ invariant and coincides with $J$ on $\mathcal{D}_{L_{f}}$. The set $V_{f}$ is defined by a holomorphic equation hence $J T V_{f} \subset T V_{f}$. We conclude that $\phi_{w} \mathcal{D}_{L_{f}} \subset \mathcal{D}_{L_{f}}$.

We have shown that the weighted sphere $S^{2 n+1}(w)$ induces a Sasaki structure on $L_{f}$. Recall that we have a principal orbibundle $\pi: S^{2 n+1}(w) \longrightarrow \mathbb{C P}^{n}(w)$ because the weights $w_{i}$ are positive integers. What we have just proven implies that the canonical inclusion $\iota: L_{f} \longrightarrow S^{2 n+1}(w)$ is a Sasakian embedding. Moreover the two structures are regular, see Example 3.65 . Hence we have the following commutative diagram

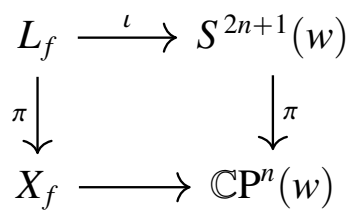


where the horizontal lines are Sasakian and Kähler embeddings respectively while the vertical arrows are principal $S^{1}$-orbibundles.

\subsection{Transverse Hodge theory}

This section is dedicated to the transverse geometry of K-contact and Sasaki manifolds. We review some properties of Sasaki manifolds which depend on the transverse Kähler structure. In particular, we focus on the basic cohomology of the Reeb foliation.

Let us begin with the definition of the basic cohomology of a foliation. Let $\mathcal{F}$ be a foliation on a smooth manifold $M$. Denote by $\Omega_{B}^{r}(\mathcal{F})$ be the set of basic $r$-forms. A form is basic if it is horizontal and invariant. Namely, a $r$-form $\alpha \in \Omega^{r}(M)$ is basic if

$$
\iota_{V} \alpha=0 \quad \text { and } \quad \iota_{V} \mathrm{~d} \alpha=0
$$

for all vector fields $V$ tangent to $\mathcal{F}$. It is clear that the exterior derivative of a basic form is again basic. Therefore, the direct sum $\Omega_{B}(\mathcal{F})=\oplus_{r} \Omega_{B}^{r}(\mathcal{F})$ is a subcomplex of the de Rham complex $\Omega(M)$. When relevant, we denote the restriction of the exterior derivative to basic forms by $d_{B}$.

Definition 3.69. The basic cohomology of the foliation $\mathcal{F}$ is the cohomology of the complex $\Omega_{B}(\mathcal{F})$.

Now let $(M, \eta, \phi, R, g)$ be a compact K-contact manifold. When the contact structure is regular $M$ is a $S^{1}$-bundle over a symplectic manifold $X$. Thus the cohomology ring of $M$ can be computed from that of $X$ via the Leray-Hirsch Theorem and the Gysin sequence of the bundle. We want to derive a generalization of the Gysin sequence for K-contact structures which are not necessarily regular, namely, we want to get a long exact sequence which gives a relation between the cohomology groups of $M$ and those of the base orbifold.

Assume $\mathcal{F}$ is the Reeb foliation of a compact $\mathrm{K}$-contact manifold $(M, \eta, \phi, R, g)$. The isometry group $\operatorname{Isom}(M, g)$ is a compact Lie group because $M$ is compact and the flow of the Reeb vector field $R$ generates a one-parameter $\operatorname{subgroup}$ in $\operatorname{Isom}(M, g)$. Therefore, the closure of the flow of $R$ in $\operatorname{Isom}(M, g)$ is a torus $T$. Let $\Omega(M)^{T}$ be the set of $T$-invariant forms on $M$, i.e. the set of forms $\beta$ such that $\mathcal{L}_{V} \beta=0$ for all vector fields $V$ tangent to $T$. Notice that $\Omega_{B}(\mathcal{F}) \subset \Omega(M)^{T}$ since the orbit of $R$ is dense in $T$. On the other hand, the form $\iota_{R} \alpha$ is basic for any $T$-invariant form $\alpha$. This follows from $\iota_{R} \iota_{R} \alpha=0$ and $\iota_{R}\left(\mathrm{~d} \iota_{R} \alpha\right)=\mathcal{L}_{R}\left(\iota_{R} \alpha\right)=0$. Therefore, we have a short sequence of complexes

$$
0 \longrightarrow \Omega_{B}^{*}(\mathcal{F}) \longrightarrow \Omega^{*}(M)^{T} \stackrel{\iota_{R}}{\longrightarrow} \Omega_{B}^{*-1}(\mathcal{F}) \longrightarrow 0 .
$$

We want to show that this sequence is exact and study the induced long exact sequence in cohomology. Exactness in the middle term follows directly from the definitions of $\Omega_{B}(\mathcal{F})$ and $\Omega(M)^{T}$. Thus we only have to prove surjectivity of the last map. For 
any form $\alpha$ we have $\iota_{R}(\eta \wedge \alpha)=\alpha$. We want to show that the form $\eta \wedge \alpha$ is $T$-invariant when $\alpha$ is basic. Let $V$ be a vector field in the Lie algebra t of $T$. Then we have

$$
\mathcal{L}_{V}(\eta \wedge \alpha)=\left(\mathcal{L}_{V} \eta\right) \wedge \alpha+\eta \wedge \mathcal{L}_{V}(\alpha) .
$$

Here the first summand vanishes because $V \in \mathrm{t}$ preserves the contact form. Moreover, the second summand vanishes because basic forms are $T$-invariant.

The cohomology of the complex $\Omega(M)^{T}$ is isomorphic to the de Rham cohomology $H^{*}(M ; \mathbb{R})$ because $T$ is compact [53, Chapter IV]. Therefore, the induced long exact sequence reads

$$
\cdots \longrightarrow H_{B}^{r}(\mathcal{F}) \longrightarrow H^{r}(M ; \mathbb{R}) \longrightarrow H_{B}^{r-1}(\mathcal{F}) \stackrel{\delta}{\longrightarrow} H_{B}^{r+1}(\mathcal{F}) \longrightarrow \cdots
$$

where the connecting morphism $\delta$ is given by $\delta[\alpha]=[\mathrm{d} \eta \wedge \alpha]=[\mathrm{d} \eta] \cup[\alpha]$.

Now let $(M, \eta, \phi, R, g)$ be a quasi-regular K-contact manifold and let $\pi: M \rightarrow \mathcal{X}$ be the associated principal $S^{1}$-orbibundle. We want to show that $\pi$ induces an isomorphism of complexes

$$
\pi^{*}: \Omega^{*}(\mathcal{X}) \longrightarrow \Omega_{B}^{*}(\mathcal{F})
$$

where $\Omega^{*}(\mathcal{X})$ is the complex of forms in the orbifold sense. Let $\beta$ be a form on $\mathcal{X}$. Clearly $\iota_{R} \pi^{*} \beta=\iota_{\pi_{*} R} \beta=0$. Moreover, the form $\pi^{*} \beta$ is invariant under the flow of $R$ because $0=\mathcal{L}_{R} \pi^{*} \beta=\iota_{R} \mathrm{~d} \pi^{*} \beta$. Thus $\pi^{*} \beta$ is a basic form on $M$. Conversely, given a basic form $\alpha$ on $M$, we can define a form $\beta$ on $\mathcal{X}$ such that $\pi^{*} \beta=\alpha$. Recall from the proof of the Structure Theorem 3.59 that the charts of $\mathcal{X}$ around $x=\pi(p)$ are given by slices $S_{x}$ for the $S^{1}$-action around the orbit $x$. The form $\beta$ is then given by the pullback of $\alpha$ under the embedding $S_{x} \hookrightarrow M$. This is independent of the choice of the point $p$ in the orbit $x$ at which we take the slice. It is clear that this is a one-to-one correspondence. Hence, for quasi-regular K-contact manifold one gets an isomorphism

$$
\pi^{*}: H_{d R}^{*}(\mathcal{X}) \cong H^{*}(X ; \mathbb{R}) \longrightarrow H_{B}^{*}(\mathcal{F})
$$

where the first isomorphism is described in Proposition 2.13

Therefore, the long exact sequence (3.9) now reads

$$
\cdots \rightarrow H^{r}(X ; \mathbb{R}) \longrightarrow H^{r}(M ; \mathbb{R}) \longrightarrow H^{r-1}(X ; \mathbb{R}) \stackrel{\delta}{\longrightarrow} H^{r+1}(X ; \mathbb{R}) \rightarrow \cdots .
$$

One clearly recovers the usual Gysin sequence with real coefficients in the case of regular compact K-contact manifolds.

The basic cohomology of compact K-contact manifolds satisfies many properties due to the existence of a transverse Hodge theory. Let us introduce the main objects involved. We begin by defining the transverse Hodge star:

$$
\bar{\star} \alpha=\star(\eta \wedge \alpha)=(-1)^{r} \iota_{R} \star \alpha
$$


where $\alpha$ is a $r$-form. Following the analogy with standard Hodge theory, we define the adjoint $\delta_{B}$ of the exterior derivative $d_{B}$ by $\delta_{B}=-\bar{\star} D_{B} \bar{\star}$. The basic Laplacian $\Delta_{B}$ is then given by $\Delta_{B}=\mathrm{d}_{B} \delta_{B}+\delta_{B} \mathrm{~d}_{B}$. We can now define the vector space $\mathcal{H}_{B}^{r}(\mathcal{F})$ of harmonic $r$-forms to be the kernel of the basic Laplacian $\operatorname{ker} \Delta_{B} \subset \Omega_{B}^{r}(\mathcal{F})$. One easily sees that $\operatorname{ker} \Delta_{B}=\operatorname{ker} \delta_{B} \cap \operatorname{ker} d_{B}$. The next theorem of El Kacimi-Alaoui and Hector [42] constitutes the transverse analogue of the Hodge Theorem and of Poincare duality.

Theorem 3.70 ([4]2]). Let $(M, \eta, \phi, R, g)$ be a compact $K$-contact manifold of dimension $2 n+1$ and $\mathcal{F}$ its Reeb foliation. Then we have

i) Each basic cohomology class admits a unique harmonic representative, i.e.

$$
\mathcal{H}_{B}^{r}(\mathcal{F}) \cong H_{B}^{r}(\mathcal{F})
$$

ii) The vector spaces $H_{B}^{r}(\mathcal{F})$ are finite dimensional and $H_{B}^{r}(\mathcal{F})=0$ for $r>2 n$.

iii) There is a non-degenerate pairing

$$
\begin{aligned}
H_{B}^{r}(\mathcal{F}) \otimes H_{B}^{2 n-r}(\mathcal{F}) & \longrightarrow \mathbb{R} \\
([\alpha],[\beta]) & \mapsto \int_{M} \eta \wedge \alpha \wedge \beta .
\end{aligned}
$$

From this theorem and the sequence (3.9) we can derive the following

Proposition 3.71. Let $(M, \eta, \phi, R, g)$ be a compact $K$-contact manifold of dimension $2 n+1$. Then

i) $H_{B}^{2 n}(\mathcal{F}) \cong \mathbb{R}$

ii) $[d \eta]$ is a non-trivial class in $H_{B}^{2}(\mathcal{F})$,

iii) the even degree basic cohomology vector spaces $H_{B}^{2 r}(\mathcal{F})$ are non-trivial and

iv) $H_{B}^{1}(\mathcal{F}) \cong H^{1}(M)$.

Proof. Recall the long exact sequence 3.9]:

$$
\cdots \longrightarrow H_{B}^{r}(\mathcal{F}) \longrightarrow H^{r}(M ; \mathbb{R}) \stackrel{\iota}{\longrightarrow} H_{B}^{r-1}(\mathcal{F}) \stackrel{\delta}{\longrightarrow} H_{B}^{r+1}(\mathcal{F}) \longrightarrow \cdots
$$

For $r=2 n+1$ the sequence gives an isomorphism $\iota: H^{2 n+1}(M ; \mathbb{R}) \rightarrow H_{B}^{2 n}(\mathcal{F})$ because $H_{B}^{r}(\mathcal{F})=0$ for $r>2 n$. This isomorphism is induced by contracting with $R$. Therefore, $\iota$ maps the class $\left[\eta \wedge(\mathrm{d} \eta)^{n}\right]$ to the basic class $\left[\mathrm{d} \eta^{n}\right]$. We conclude that $[\mathrm{d} \eta]$ is a non-trivial class. Moreover, since $\left[\mathrm{d} \eta^{n}\right]$ generates $H_{B}^{2 n}(\mathcal{F})$ we have $\left[\mathrm{d} \eta^{r}\right] \neq 0$ for all $0<r<n+1$.

Now consider the sequence (3.9) for $r=1$, i.e.

$$
0 \longrightarrow H_{B}^{1}(\mathcal{F}) \longrightarrow H^{1}(M ; \mathbb{R}) \stackrel{\iota}{\longrightarrow} \mathbb{R} \stackrel{\delta}{\longrightarrow} H_{B}^{2}(\mathcal{F}) \longrightarrow \cdots
$$


Recall that the connecting morphism $\delta$ maps a class $[\alpha]$ to $[\mathrm{d} \eta \wedge \alpha]=[\mathrm{d} \eta] \cup[\alpha]$. Since $[\mathrm{d} \eta] \neq 0$, the connecting morphism $\delta$ is injective in degree 0 . Hence we get an isomorphism $H_{B}^{1}(\mathcal{F}) \cong H^{1}(M ; \mathbb{R})$.

Remark 3.72. If the K-contact structure is quasi-regular the previous theorem holds when replacing basic cohomology by orbifold cohomology.

We can then define some transverse invariants of K-contact structures which originate from basic cohomology.

Definition 3.73. Let $(M, \eta, \phi, R, g)$ be a compact K-contact manifold of dimension $2 n+1$ and $\mathcal{F}$ its Reeb foliation. The $r$-th basic Betti number $\mathrm{b}_{r}^{B}(\mathcal{F})$ is the dimension of the $r$-th basic cohomology vector space, i.e.

$$
\mathrm{b}_{r}^{B}(\mathcal{F})=\operatorname{dim}\left(H_{B}^{r}(\mathcal{F})\right) .
$$

Similarly, we define the basic Euler characteristic $\chi(\mathcal{F})$ to be

$$
\chi(\mathcal{F})=\sum_{r=0}^{2 n}(-1)^{r} \operatorname{dim}\left(H_{B}^{r}(\mathcal{F})\right) .
$$

It was proven in [43] that if $f:(M, \eta, \phi, R, g) \rightarrow\left(M, \eta^{\prime}, \phi^{\prime}, R^{\prime}, g^{\prime}\right)$ is a K-contact transformation, then $f$ induces a ring isomorphism $f^{*}: H_{B}^{*}\left(\mathcal{F}^{\prime}\right) \rightarrow H_{B}^{*}(\mathcal{F})$. That is, the basic cohomology of a K-contact manifold is invariant under K-contact transformations. A stronger result holds when the K-contact structure is Sasaki. Namely, the basic cohomology of a Sasaki manifold $M$ is a topological invariant of $M$, see Theorem 5.2 .

Let us move on to Hodge theory for Sasaki manifolds. Assume $(M, \eta, \phi, R, g)$ to be a Sasaki manifold for the remainder of this section. We consider the complexification $\mathcal{D}_{\mathbb{C}}=\mathcal{D} \otimes \mathbb{C}$ of the contact distribution $\mathcal{D}$. Since $\left(\mathcal{D}, \phi_{\left.\right|_{\mathcal{D}}}\right)$ is a complex bundle, $\phi_{\left.\right|_{\mathcal{D}}}$ has eigenvalues $i$ and $-i$. Denote by $\mathcal{D}_{1,0}$, respectively $\mathcal{D}_{0,1}$, the eigenspace relative to the eigenvalue $i$, resp. $-i$. Now let $\mathcal{D}^{1,0}$, resp. $\mathcal{D}^{0,1}$, be their duals. Then we can define the basic forms of type $(p, q)$ to be sections of the bundle $\left(\bigwedge^{p} \mathcal{D}^{1,0}\right) \wedge\left(\bigwedge^{q} \mathcal{D}^{0,1}\right)$. The set of basic $(p, q)$-forms will be denoted by $\Omega^{p, q}(\mathcal{F})$. In analogy with the almost complex case, we can define the operators $\partial$ and $\partial$. Moreover, the integrability of the CR structure yields $\mathrm{d}_{B}=\partial+\bar{\partial}$.

Definition 3.74. The complex $\left(\Omega^{\bullet \bullet \bullet}(\mathcal{F}), \bar{\partial}\right)$ is called the basic Dolbeault complex and its cohomology $H^{\bullet, \bullet}(\mathcal{F})$ is the basic Dolbeault cohomology of the Sasaki manifold $(M, \eta, \phi, R, g)$. The basic Hodge numbers $h_{B}^{p, q}(\mathcal{F})$ are defined to be

$$
h_{B}^{p, q}(\mathcal{F})=\operatorname{dim}\left(H^{p, q}(\mathcal{F})\right) .
$$

Notation. When the Sasaki structure on a manifold $M$ is understood we will write $h_{B}^{p, q}(M)$ instead of $h_{B}^{p, q}(\mathcal{F})$. 
Remark 3.75. If the Sasaki structure is quasi-regular, the basic Dolbeault complex is the Dolbeault complex of the orbifold $\mathcal{X}$.

Since the Reeb foliation of a Sasaki manifold is transversally Kähler, it is natural to ask whether the basic Dolbeault cohomology of a Sasaki manifold enjoys some of the properties of the Dolbeaut cohomology of a Kähler manifold. The following theorems of El Kacimi-Alaoui [41] give a positive answer to this question. In particular El Kacimi-Alaoui's results provide a relation between basic Dolbeault cohomology and the cohomology $H_{B}^{*}(\mathcal{F} ; \mathbb{C})$ of the complex of basic complex valued forms $\Omega_{B}^{\mathbb{C}}(\mathcal{F})$. In order to lighten the notation, we will not specify the coefficients when they are clear from the context.

The first theorem that we present collects some results from [41] among which the transverse versions of the Hodge decomposition and of Serre duality.

Theorem 3.76 ([41] $)$. Let $(M, \eta, \phi, R, g)$ be a compact Sasaki manifold of dimension $2 n+1$. Then

i) $H^{n, n}(\mathcal{F}) \cap H_{B}^{2 n}(\mathcal{F}) \cong \mathbb{R}$.

ii) The basic form $d \eta$ is of pure type $(1,1)$, i.e. $[d \eta] \in H^{1,1}(\mathcal{F}) \cap H_{B}^{2}(\mathcal{F})$.

iii) The group $H^{r, r}(\mathcal{F})$ is non-trivial for all $0<r<n+1$.

iv) Complex conjugation induces an isomorphism $H^{p, q}(\mathcal{F}) \cong H^{q, p}(\mathcal{F})$.

v) There is a decomposition $H_{B}^{r}(\mathcal{F} ; \mathbb{C}) \cong \bigoplus_{p+q=r} H^{p, q}(\mathcal{F})$.

vi) The odd degree basic cohomology vector space $H_{B}^{2 r+1}(\mathcal{F})$ is even dimensional for $r<n$.

vii) There is an isomorphism $H^{p, q}(\mathcal{F}) \cong H^{n-p, n-q}(\mathcal{F})$.

Corollary 3.77. The basic Hodge and Betti numbers of a Sasaki manifold satisfy the following relations

$$
h_{B}^{p, q}(\mathcal{F})=h_{B}^{n-p, n-q}(\mathcal{F})=h_{B}^{q, p}(\mathcal{F}), \quad b_{B}^{r}(\mathcal{F})=\sum_{p+q=r} h_{B}^{p, q}(\mathcal{F}) .
$$

In analogy with the Kähler case, the basic operator $L: \Omega_{B}^{r}(\mathcal{F}) \longrightarrow \Omega_{B}^{r+2}(\mathcal{F})$ is defined by

$$
L \alpha=\alpha \wedge \mathrm{d} \eta
$$

Its adjoint $\Lambda: \Omega_{B}^{r}(\mathcal{F}) \longrightarrow \Omega_{B}^{r-2}(\mathcal{F})$ is therefore given by

$$
\Lambda=-\bar{\star} L \bar{\star} \text {. }
$$

The proof of the standard case applies mutatis mutandis to prove Theorem 3.76. In particular, we define the basic primitive cohomology group $P^{r}(\mathcal{F})$ as the kernel of the map induced by $\Lambda$ in cohomology. 
Another result in [41] which is relevant to our discussion is the transverse Hard Lefschetz Theorem.

Theorem 3.78 ([41]). Let $(M, \eta, \phi, R, g)$ be a compact Sasaki manifold of dimension $2 n+1$ and let $L$ be the operator induced in cohomology by (3.12). Then $L^{k}: H_{B}^{n-k}(\mathcal{F}) \rightarrow$ $H_{B}^{n+k}(\mathcal{F})$ is an isomorphism for $1 \leqslant k \leqslant n$. Moreover there is a decomposition

$$
H_{B}^{r}(\mathcal{F})=\bigoplus_{k \geqslant 0} L^{k} P^{r-2 k}(\mathcal{F}) .
$$

Remark 3.79. Let $(M, \eta, \phi, R, g)$ be a compact quasi-regular Sasaki manifold of dimension $2 n+1$. By the Structure Theorem, $M$ is the total space of a principal $S^{1}$-orbibundle $M \stackrel{\pi}{\rightarrow} \mathcal{X}$ over a Kähler orbifold. Thus the basic and Dolbeault cohomology of the Reeb foliation are isomorphic to the de Rham and Dolbeault cohomology of $\mathcal{X}$. Since the isomorphism is given by $\pi^{*}$, the Kähler class $[\omega]$ maps to [d $\left.\eta\right]$.

If the structure on $M$ is regular, then the basic forms agree with pullbacks of forms on the base of the $S^{1}$-bundle $M \stackrel{\pi}{\rightarrow} X$ where now $\mathrm{X}$ is a smooth projective variety. Then the basic and Dolbeault cohomology are isomorphic to the standard de Rham and Dolbeault cohomologies. In this case the transverse Hodge decomposition and transverse Hard Lefschetz Theorem reduce to the standard ones.

Let us now discuss further transverse invariants of Sasaki structures. Consider the contact distribution $\mathcal{D}$ and its Chern classes. We can compute representatives of the Chern classes of $\mathcal{D}$ via Chern-Weil theory with the use of a connection $\nabla^{\mathcal{D}}$ on $\mathcal{D}$. We consider a connection $\nabla$ given by taking the projection of the Levi-Civita connection of $g$ onto $\mathcal{D}$. When restricting $\nabla$ to $\mathcal{D}$, we get a connection $\nabla^{\mathcal{D}}$ on $\mathcal{D}$. This is compatible with the transverse Kähler metric $g_{\mathcal{D}}$ and torsion-free by definition. Moreover $\mathcal{L}_{R} \nabla^{\mathcal{D}}=0$ because the metric is compatible with $g_{\mathcal{D}}$ and $R$ is Killing. Therefore, the connection 1form of $\nabla^{\mathcal{D}}$ is basic because $\iota_{R} \nabla^{\mathcal{D}}=0$, see [9, Lemma 6.2]. Hence, the curvature 2-form $\Omega$ of $\nabla$ is also basic. Moreover, since the Reeb foliation is transversally holomorphic, $\Omega$ is a basic $(1,1)$-form. Chern-Weil theory then implies that the $i$-th Chern class $c_{i}(\mathcal{D}) \in$ $H^{2 i}(M, \mathbb{Z})$ of $\mathcal{D}$ is represented by a basic $(i, i)$-form $\alpha_{i}$.

Definition 3.80. Let $(M, \eta, \phi, R, g)$ be a compact Sasaki manifold. The $i$-th basic Chern class $c_{i}(\mathcal{F})$ is defined to be the class $\left[\alpha_{i}\right] \in H^{i, i}(\mathcal{F})$ defined above.

Remark 3.81. Let $(M, \eta, \phi, R, g)$ be a compact (quasi-)regular Sasaki manifold and $M \stackrel{\pi}{\rightarrow}$ $X$ the associated principal $S^{1}$-(orbi)bundle. Then the basic Chern classes of $M$ are identified with the (orbifold) Chern classes of the tangent bundle of $X$.

Lemma 3.82. Let $(M, \eta, \phi, R, g)$ be a compact Sasaki manifold. Then the basic Chern classes map to the Chern classes of $\mathcal{D}$ under the natural inclusion $H^{i, i}(\mathcal{F}) \rightarrow H^{2 i}(M)$. In particular, if $M$ is quasi-regular with associated principal $S^{1}$-orbibundle $M \stackrel{\pi}{\rightarrow} \mathcal{X}$, then $\pi^{*} c_{i}^{\text {orb }}(\mathcal{X})=c_{i}(\mathcal{F})$.

Proof. Both statements follow directly from the construction of basic Chern classes. 
We conclude this section with the definition of type for Sasaki structures.

Definition 3.83. Let $(M, \eta, \phi, R, g)$ be a compact Sasaki manifold. The Sasaki structure is of positive type, respectively negative type, if the first basic Chern class $c_{1}(\mathcal{F})$ can be represented by a positive, resp. negative, definite $(1,1)$-form. The structure will be called null if $c_{1}(\mathcal{F})=0$. In these cases the Sasaki structure is definite. Otherwise it is called indefinite.

Notation. We often simply say that a Sasaki structure of positive, respectively negative, type is positive, resp. negative. When we refer to the type of a definite Sasaki structure we are considering whether it is positive, negative or null.

Example 3.84. Let $X$ be a Kähler manifold with ample canonical bundle $K_{X}$. By the Kodaira Embedding Theorem $X$ is a projective manifold and the canonical class $K_{X}$, or any positive multiple of it, can be represented by a Kähler form $\omega$. Now the BoothbyWang bundle $M$ over $(X, \omega)$ is a regular Sasaki manifold whose first basic Chern class is exactly $c_{1}(X)=-K_{X}$, that is, a negative multiple of a Kähler class. Therefore $M$ is a negative Sasaki manifold. Summarizing, given a Kähler manifold with ample canonical bundle we can associate a negative Sasaki manifold to each choice of a Kähler form representing a negative multiple of $c_{1}(X)$.

Links provide a class of Sasaki manifolds that are always of definite type, see Example 3.66. The following proposition gives a simple numerical condition for the type of the standard Sasaki structure on a link. We use here the notation from Example 3.66

Proposition 3.85 ([16]). Let $L_{f} \subset S^{2 n+1}(w)$ be a link. Then the basic first Chern class is a multiple of $\left[d \eta_{w}\right]_{B}$ and the Sasaki structure is

i) positive if and only if $\sum w_{i}-d>0$,

i) negative if and only if $\sum w_{i}-d<0$,

i) null if and only if $\sum w_{i}-d=0$.

\subsection{The join construction}

In the Kähler setting there exists a straightforward way to produce new Kähler manifolds from known ones. Namely, the product $M_{1} \times M_{2}$ of two Kähler manifolds $\left(M_{1}, \omega_{1}\right)$ and $\left(M_{2}, \omega_{2}\right)$ can be endowed with the Kähler forms $k_{1} \omega_{1}+k_{2} \omega_{2}$ for parameters $k_{1}, k_{2}>0$. Clearly the product of two Sasakian manifolds cannot be Sasakian for dimension reasons. The goal of this section is to present a construction of Sasaki manifolds introduced in [17]. This operation, called the join construction, plays the role of products in the Sasaki setting.

Remark 3.86. The results in this section will be proved for Sasaki manifolds but they hold true in the K-contact setting. Therefore we will give the definitions and the statements for both cases. 
Definition 3.87. Let $(M, \eta, \phi, R, g)$ be a quasi-regular contact manifold such that the leaves of the Reeb foliation are compact, e.g. $M$ is compact. The order $v(M, \eta, \phi, R, g)$ of the contact manifold $M$ is the least common multiple of the orders of the isotropy groups of the $S^{1}$-action on $M$. We will write $v(M)$ when the contact structure is understood.

Now let $\left(M_{1}, \eta_{1}, \phi_{1}, R_{1}, g_{1}\right)$ and $\left(M_{2}, \eta_{2}, \phi_{2}, R_{2}, g_{2}\right)$ be two compact quasi-regular Sasaki manifolds. By the Structure Theorem 3.59, $M_{i}$ is a principal $S^{1}$-orbibundle over a Kähler orbifold $\mathcal{X}_{i}$ with Kähler class $\left[\omega_{i}\right] \in H_{\text {orb }}^{2}\left(\mathcal{X}_{i} ; \mathbb{Z}\right)$. For all integers $k_{1}, k_{2}>0$ the form $k_{1} \omega_{1}+k_{2} \omega_{2}$ is an integral Kähler form on the orbifold $\mathcal{X}_{1} \times \mathcal{X}_{2}$. Therefore, the class $\left[k_{1} \omega_{1}+k_{2} \omega_{2}\right] \in H_{\text {orb }}^{2}\left(\mathcal{X}_{1} \times \mathcal{X}_{2} ; \mathbb{Z}\right)$ determines a principal $S^{1}$-orbibundle

$$
\pi: M_{1} \star_{k_{1}, k_{2}} M_{2} \longrightarrow \mathcal{X}_{1} \times \mathcal{X}_{2}
$$

By the Structure Theorem 3.59, $M_{1} \star_{k_{1}, k_{2}} M_{2}$ has a Sasaki structure determined by a connection 1-form with curvature $\pi^{*}\left(k_{1} \omega_{1}+k_{2} \omega_{2}\right)$. Note that the total space of this orbibundle is in general an orbifold and not a smooth manifold.

Definition 3.88. The $\left(k_{1}, k_{2}\right)$-join of two compact quasi-regular Sasaki, respectively K-contact, manifolds $\left(M_{1}, \eta_{1}, \phi_{1}, R_{1}, g_{1}\right)$ and $\left(M_{2}, \eta_{2}, \phi_{2}, R_{2}, g_{2}\right)$ is the Sasaki, resp. Kcontact, orbifold $M_{1} \star_{k_{1}, k_{2}} M_{2}$ constructed above.

Since we are interested in constructing smooth Sasaki manifolds, we characterize the pairs $\left(k_{1}, k_{2}\right)$ for which $M_{1} \star_{k_{1}, k_{2}} M_{2}$ is a smooth manifold. In order to do so consider the manifold $M_{1} \times M_{2}$ as a torus orbibundle bundle over $\mathcal{X}_{1} \times \mathcal{X}_{2}$. Notice that the total space $M_{1} \star_{k_{1}, k_{2}} M_{2}$ can be seen as the quotient of $M_{1} \times M_{2}$ by a circle action given by

$$
\begin{aligned}
S^{1} \times M_{1} \times M_{2} & \longrightarrow M_{1} \times M_{2} \\
\left(e^{i \theta}, x, y\right) & \longmapsto\left(x e^{i k_{2} \theta}, y e^{-i k_{1} \theta}\right)
\end{aligned}
$$

Therefore, the orbifold structure on $M_{1} \star_{k_{1}, k_{2}} M_{2}$ is trivial if and only if the circle action above is free. Let $(x, y)$ be a point in $M_{1} \times M_{2}$ and denote by $v_{x}$, respectively $v_{y}$, the order of the isotropy group of $x$, resp $y$, for the $S^{1}$-action on the compact quasiregular Sasaki manifold $M_{1}$, resp. $M_{2}$. Clearly the isotropy subgroup at $(x, y)$ has order $v_{(x, y)}=\operatorname{gcd}\left(v_{x} k_{2}, v_{y} k_{1}\right)$. In conclusion, the circle action on $M_{1} \times M_{2}$ is free if and only if $\operatorname{gcd}\left(v_{x} k_{2}, v_{y} k_{1}\right)=1$ for all $(x, y) \in M_{1} \times M_{2}$.

A notable case is when both $M_{1}$ and $M_{2}$ are compact regular Sasaki manifolds. In this case $M_{1} \star_{k_{1}, k_{2}} M_{2}$ is a regular Sasaki manifold whenever $k_{1}$ and $k_{2}$ are relatively prime, e.g. when $k_{1}=k_{2}=1$. For this reason we give the following

Definition 3.89. The join $M_{1} \star M_{2}$ of two compact regular Sasaki or K-contact manifolds is the $(1,1)$-join $M_{1} \star_{1,1} M_{2}$.

We close this section with a characterization of the join in terms of the $S^{1}$-orbibundle associated to the Sasaki structures. 
Proposition 3.90. Let $M_{1} \star_{k_{1}, k_{2}} M_{2}$ be the smooth join of two compact quasi-regular Sasaki or K-contact manifolds $M_{1}$ and $M_{2}$. Then $M_{1} \star_{k_{1}, k_{2}} M_{2}$ is a bundle over $\mathcal{X}_{1}$ with fiber $M_{2} / \mathbb{Z}_{k_{2}}$ associated to the orbibundle $M_{1} \rightarrow \mathcal{X}_{1}$.

Proof. $M_{1} \star_{k_{1}, k_{2}} M_{2}$ is the quotient of $M_{1} \times M_{2}$ by the circle action (3.14). Let the subgroup $\mathbb{Z}_{k_{2}} \subset S^{1}$ act first to obtain the quotient $M_{1} \times M_{2} / \mathbb{Z}_{k_{2}}$. Denote a point in $M_{1} \times M_{2} / \mathbb{Z}_{k_{2}}$ by $(x,[y])$. Now $M_{1} \star_{k_{1}, k_{2}} M_{2}$ is the quotient of $M_{1} \times M_{2} / \mathbb{Z}_{k_{2}}$ by the diagonal $S^{1}$-action where $S^{1}=S^{1} / \mathbb{Z}_{k_{2}}$ acts by $(x,[y]) e^{i \theta}=\left(x e^{i \theta},\left[y e^{-i k_{k_{2}}} \theta\right]\right)$. By definition this is the $M_{2} / \mathbb{Z}_{k_{2}}$-bundle associated to $M_{1} \rightarrow \mathcal{X}_{1}$.

Corollary 3.91. Let $M_{1} \star M_{2}$ be the join of two compact regular Sasaki or K-contact manifolds $M_{1}$ and $M_{2}$. Denote by $M \rightarrow X_{1}$ and $M_{2} \rightarrow X_{2}$ the associated Boothby-Wang fibrations. Then $M_{1} \star M_{2}$ is a $M_{2}$-bundle over $X_{1}$.

Remark 3.92. The roles of the compact regular Sasaki manifolds $M_{1}$ and $M_{2}$ are interchangeable. Hence, $M_{1} \star M_{2}$ is a $M_{1}$-bundle over $X_{2}$.

Remark 3.93. The join construction for K-contact manifolds is a special case of the contact fiber bundles by Lerman, see [83].

\subsection{Topology of Sasakian manifolds}

We conclude this chapter by reviewing some of the topological properties of Sasakian manifolds.

We begin by showing that in dimension 3 every K-contact structure is Sasaki. This is a consequence of the fact that every almost complex structure on a surface is integrable.

Theorem 3.94. A 3-dimensional $K$-contact manifold $(M, \eta, \phi, R, g)$ is Sasaki.

Proof. By Corollary 3.47, we have to show that the induced almost CR structure is integrable. Let $\mathcal{H}=\{X-i J X \mid X \in \mathcal{D}\}$ as in Section 3.5. We want to prove the integrability condition

$$
[X-i \phi X, Y-i \phi Y] \in \mathcal{H} \text { for all } X, Y \in \mathcal{D} .
$$

By Proposition 3.41 the tensor $N^{(2)}$ vanishes. Thus we get $N^{(2)}(\phi X, Y)=\eta([\phi X, Y]+$ $[X, \phi Y])=0$ for $X, Y \in \mathcal{D}$. Thus 3.15 ) is equivalent to

$$
\phi[X, Y]-\phi[\phi X, \phi Y]-[\phi X, Y]-[X, \phi Y]=0 \text { for all } X, Y \in \mathcal{D} .
$$

The equation above is easily verified by choosing a basis of $\mathcal{D}$ of the form $\{X, Y=$ $\phi X\}$.

We now turn our attention to Sasaki manifolds of higher dimension. It is very natural to ask which topological properties of Kähler manifolds are enjoyed by Sasakian manifolds. 
We begin by investigating formality. For the basic material we refer the reader to [44]. Consider a commutative differential graded algebra $\left(A, \mathrm{~d}_{A}\right)$ (CDGA $A$ for short) over $\mathbb{R}$. We denote by $|a|$ the degree of an element $a \in A$ and by $H^{*}(A)$ the cohomology of the complex $\left(A, \mathrm{~d}_{A}\right)$. The CDGA we mainly focus on is the de Rham complex of a Sasakian manifold.

A CDGA $A$ is called minimal if

1) $A$ is the free algebra $\bigwedge V$ over a graded vector space $V$.

2) There exists a set of generators $\left\{a_{i}\right\}$, indexed by a well-ordered set, such that

i) $\left|a_{i}\right| \leqslant\left|a_{j}\right|$ for $i<j$ and

ii) $\mathrm{d}_{A} a_{i}$ is expressed in terms of $a_{j}$ for $j<i$.

A morphism of CDGA's is then a morphism of algebras that commutes with the differential and respects the grading. A quasi-isomorphism of CDGA's is a morphism which induces an isomorphism in cohomology. More generally, we can define the notion of weak equivalence. Namely, two commutative differential graded algebras $A$ and $B$ are said to be weakly equivalent if there is a sequence of quasi-isomorphisms

$$
A \longleftarrow C_{1} \longrightarrow C_{2} \longleftarrow \cdots \longleftarrow C_{n} \longrightarrow B .
$$

An elementary extension of a CDGA $\left(A, \mathrm{~d}_{A}\right)$ is a CDGA of the form $\left(B=A \otimes \wedge V, \mathrm{~d}_{B}\right)$ satisfying the following properties:

i) $V$ is finite-dimensional and all elements of $V$ have the same degree.

ii) $\mathrm{d}_{B}(a)=\mathrm{d}_{A}(a)$ for all $a \in A$ and $\mathrm{d}_{B}(v) \in A$ for all $v \in V$.

A minimal model for a CDGA $A$ is a minimal commutative differential graded algebra $\bigwedge V$ together with a quasi-isomorphism $\rho: \bigwedge V \longrightarrow A$. A CDGA is called connected if its 0-th cohomology group is isomorphic to $\mathbb{R}$. Every connected CDGA admits a minimal model which is unique up to isomorphism, see [58]. Therefore, weakly equivalent connected CDGA's have isomorphic minimal models.

Definition 3.95. Let $M$ be a connected manifold and $\bigwedge V$ the minimal model for its de Rham complex $\Omega(M)$. Consider the cohomology $H_{d R}^{*}(M)$ as a CDGA with trivial differential. The manifold $M$ is formal if $\bigwedge V$ is a minimal model for $H_{d R}^{*}(M)$. Equivalently, $M$ is formal if there exists a morphism of CDGA's $\psi: \bigwedge V \longrightarrow H_{d R}^{*}(M)$ inducing an isomorphism in cohomology.

Kähler manifolds form a class of manifolds which enjoys formality [33]. It is then natural to ask whether Sasakian manifolds are formal. The first results in this direction were given by Tievsky in his Ph.D. thesis [113]. We briefly review his results.

Let $(M, \eta, \phi, R, g)$ be a compact Sasaki manifold. Consider the operator $\mathrm{d}_{B}^{c}=i(\bar{\partial}-\partial)$ on complex valued basic forms. Let $\Omega_{B}^{c}(\mathcal{F})$ be the complex of $\mathrm{d}^{c}$-closed forms with 
differential given by $\mathrm{d}_{B}$ and $H_{B}^{c}(\mathcal{F})$ its cohomology. Let $V=\langle y\rangle$ be a graded vector space with $|y|=1$. An elementary extension by $\bigwedge V$ is then determined by defining $\mathrm{d} y$. Now one can define morphisms relating an elementary extension of the basic $\mathrm{d}^{c}-$ cohomology with the de Rham complex. Namely, consider

$$
\begin{gathered}
\left(\Omega_{B}^{c}(\mathcal{F}) \otimes \wedge V, \mathrm{~d} y=\mathrm{d} \eta\right) \stackrel{\tau}{\longrightarrow}\left(H_{B}^{c}(\mathcal{F}) \otimes \wedge V, \mathrm{~d} y=[\mathrm{d} \eta]\right) \\
\alpha+\beta \otimes y \longmapsto[\alpha]+[\beta] \otimes y .
\end{gathered}
$$

Moreover, we can define the morphism

$$
\begin{aligned}
\left(\Omega_{B}^{c}(\mathcal{F}) \otimes\right. & \wedge V, \mathrm{~d} y=\mathrm{d} \eta) \stackrel{\sigma}{\longrightarrow}(\Omega(M), \mathrm{d}) \\
\alpha+\beta \otimes y & \longmapsto \alpha+\beta \wedge \eta .
\end{aligned}
$$

The main result in [113] is the following:

Theorem 3.96 ([113]). Let $(M, \eta, \phi, R, g)$ be a compact Sasaki manifold. Then the diagram

$$
\left(H_{B}^{c}(\mathcal{F}) \otimes \wedge V, d y=[d \eta]\right) \stackrel{\tau}{\longleftarrow}\left(\Omega_{B}^{c}(\mathcal{F}) \otimes \wedge V, d y=d \eta\right) \stackrel{\sigma}{\longrightarrow}(\Omega(M), d)
$$

is a weak equivalence of CDGA's.

Moreover, $\left(H_{B}^{c}(\mathcal{F}) \otimes \bigwedge V, \mathrm{~d} y=[\mathrm{d} \eta]\right)$ is isomorphic to $\left(H_{B}(\mathcal{F}) \otimes \wedge V, \mathrm{~d} y=[\mathrm{d} \eta]\right)$. Therefore, the minimal model of $\left(H_{B}(\mathcal{F}) \otimes \wedge V, \mathrm{~d} y=[\mathrm{d} \eta]\right)$ is isomorphic to the minimal model of $\left(H_{d R}(M), 0\right)$. A compact contact manifold satisfying this condition will be called a Tievsky type manifold.

Generally, determining whether a manifold is formal is a difficult problem. An important tool in detecting non-formality is given by Massey products. Massey products are indeed an obstruction to formality, a proof of this fact can be found in [33] in the discussion after Theorem 4.1. Using this result, it is easy to see that there exist non-formal Sasakian manifolds. In fact the Boothby-Wang fibration over a torus $\left(T^{2}\right)^{n}$ with standard Kähler class gives such a manifold. In [10] Biswas, Fernández, Muñoz and Tralle proved that all higher order Massey products vanish on Sasakian manifolds. Hence only triple Massey products can detect non-formality.

Another important topological property of Sasakian manifolds is the Hard Lefschetz Theorem which was proven in the Sasaki setting by Cappelletti Montano, De Nicola and Yudin.

Theorem $3.97([25])$. Let $(M, \eta, \phi, R, g)$ be a compact Sasaki manifold of dimension 
$2 n+1$. Then for $0 \leqslant r \leqslant n$ there exists an isomorphism

$$
\begin{aligned}
L^{r}: H^{n-r}(M) & \longrightarrow H^{n+r+1}(M) \\
{[\beta] } & \mapsto\left[\eta \wedge(d \eta)^{r} \wedge \beta\right]
\end{aligned}
$$

where $\beta$ is the unique harmonic representative of its cohomology class. Moreover, the isomorphism is independent of the Sasaki metric.

A compact contact manifold with such an isomorphism is called a Lefschetz contact manifold. An example of Lefschetz K-contact manifold of Tievsky type which does not admit a Sasaki structure was given in [24]. In this case the obstruction is given by the fundamental group.

As in the Kähler case, the class of fundamental groups of compact Sasakian manifolds satisfies several properties. For instance, we have the following:

Proposition 3.98. The abelianization $H_{1}(M)$ of the fundamental group $\pi_{1}(M)$ of a $S a$ sakian manifold $M$ has even rank.

Proof. This follows directly from part iv) in Proposition 3.71 and Corollary 3.77 on the basic Hodge numbers of a Sasaki manifold $M$. Namely, for any Sasaki structure on $M$ the basic Hodge numbers satisfy $h^{1,0}(\mathcal{F})=h^{0,1}(\mathcal{F})=\frac{1}{2} \mathrm{~b}_{1}(\mathcal{F})=\frac{1}{2} \mathrm{~b}_{1}(M)$.

Fundamental groups of compact Sasakian manifolds are known to meet further constraints. Since such groups are the subject of Chapter 6, we review the literature on Sasaki groups in detail in Section 4.3 . 


\section{Chapter 4}

\section{Group extensions and Kähler groups}

As a consequence of the Structure Theorem 3.59, the topology of Sasakian manifolds is closely related to the topology of Kähler orbifolds. In particular, fundamental groups of compact Sasakian manifolds can be described in terms of projective orbifold fundamental groups. In turn, these groups are related to projective groups. Hence, we explain here these relations in order to discuss fundamental groups of compact Sasakian manifolds.

\subsection{Group cohomology and central extensions}

In this section we recall the definition of group cohomology and discuss group extensions. The proofs which are omitted in this section, as well as a detailed discussion on these topics, can be found in [19, 81, 108].

Given a group $\Gamma$ a connected aspherical space $B \Gamma$ with $\pi_{1}(B \Gamma)=\Gamma$ is a classifying space for $\Gamma$. The classifying space is determined by $\Gamma$ up to homotopy equivalence. There are several construction of classifying spaces. Since we are only interested in fundamental groups of compact manifolds, we present below one such construction which is particularly convenient for us.

The space $B \Gamma$ is classifying for $\Gamma$ in the following sense. Any homomorphism of groups $\Gamma \longrightarrow \Delta$ is induced by a map $B \Gamma \longrightarrow B \Delta$ unique up to homotopy. Therefore, the homotopy type of $B \Gamma$ is determined uniquely by $\Gamma$.

We define the group cohomology $H^{*}(\Gamma ; R)$ of $\Gamma$ to be the cohomology $H^{*}(B \Gamma ; R)$ for a ring $R$.

Example 4.1 (Finite cyclic groups). A classifying space for $\Gamma=\mathbb{Z}_{n}$ is given by the infinite dimensional lens space $L(\infty, n)$. Namely, the quotient of $S^{\infty} \subset \mathbb{C}^{\infty}$ by the standard action of $\mathbb{Z}_{n}$. Clearly $\pi_{1}(L(\infty, n))=\mathbb{Z}_{n}$. Moreover, $L(\infty, n)$ is aspherical 
because its universal covering space $S^{\infty}$ is contractible. Thus we have

$$
H^{k}\left(\mathbb{Z}_{n} ; \mathbb{Z}\right)= \begin{cases}\mathbb{Z}, & \text { if } k=0 \\ \mathbb{Z}_{n}, & \text { for } k>0 \text { even } \\ 0, & \text { otherwise. }\end{cases}
$$

Example 4.2 (Surface groups). Let $\Gamma_{g}$ be the fundamental group of a closed oriented surface $\Sigma_{g}$ of genus $g \geqslant 2$. Then $\Sigma_{g}$ is the classifying space for $\Gamma_{g}$ because its universal covering is a hyperbolic disk. We conclude that $H^{*}\left(\Gamma_{g} ; \mathbb{Z}\right) \cong H^{*}\left(\Sigma_{g} ; \mathbb{Z}\right)$. The groups $\Gamma_{g}$ are called surface groups.

Example 4.3 (Free groups). Let $F_{n}$ be the free group on $n$ generators. Then $F_{n}$ is the fundamental group of a wedge of $n$ circles $\bigvee n S^{1}$. Moreover, $\bigvee n S^{1}$ has trivial higher homotopy groups. Hence $\bigvee n S^{1}=B F_{n}$. It follows that the cohomology of $F_{n}$ is trivial in degree larger than 1.

We present now an alternative defintion of group cohomology. In certain situations this will turn out to be more suitable than the definition given above.

Let $\Gamma$ be a group and $R$ a $\Gamma$-module. Consider the group $\operatorname{Hom}\left(\Gamma^{r}, R\right)$ of homogeneous homomorphisms from the $r$-fold direct product $\Gamma \times \cdots \times \Gamma$ to $R$. In other words, consider the group of homomorphisms

$$
\varphi: \Gamma \times \cdots \times \Gamma \longrightarrow R
$$

such that

$$
\gamma \varphi\left(\gamma_{1}, \ldots, \gamma_{r}\right)=\varphi\left(\gamma \gamma_{1}, \ldots, \gamma \gamma_{r}\right)
$$

for $\gamma, \gamma_{1}, \ldots, \gamma_{r} \in \Gamma$. Now consider the map d: $\operatorname{Hom}\left(\Gamma^{\bullet}, R\right) \longrightarrow \operatorname{Hom}\left(\Gamma^{\bullet+1}, R\right)$ defined by

$$
\mathrm{d} \varphi\left(\gamma_{1}, \ldots, \gamma_{r+1}\right)=\sum_{i=1}^{r+1}(-1)^{i+1} \varphi\left(\gamma_{1}, \ldots, \hat{\gamma}_{i}, \ldots, \gamma_{r+1}\right)
$$

where the notation $\hat{\gamma}_{i}$ means that the $i$-th entry is omitted. Then $\left(\operatorname{Hom}\left(\Gamma^{\bullet}, R\right), \mathrm{d}\right)$ is a cochain complex. One can show that the group cohomology $H^{*}(\Gamma, R)$ is isomorphic to the cohomology of the complex $\left(\operatorname{Hom}\left(\Gamma^{\bullet+1}, R\right), \mathrm{d}\right)$. Let us present an instance in which this definition is more convenient for computations.

Example 4.4. From the above description follows that the cohomology of a torsion group of order $m$ vanishes if the coefficients are divisible by $m$. For simplicity let us consider real coefficients. Namely, let $\Gamma$ be a group such that $\gamma^{m}=0$ for all $\gamma \in \Gamma$ and consider $\mathbb{R}$ as a trivial $\Gamma$-module. It is clear that every homomorphism $\varphi: \Gamma \times \cdots \times \Gamma \longrightarrow \mathbb{R}$ satisfies $m \varphi=0$. Therefore, we have $m H^{r}(\Gamma ; \mathbb{R})=0$ for all $r>0$. On the other hand $m H^{r}(\Gamma ; \mathbb{R})=H^{r}(\Gamma ; m \mathbb{R})=H^{r}(\Gamma ; \mathbb{R})=0$. Hence we have $\widetilde{H}^{*}(\Gamma ; \mathbb{Z})=0$.

Consider now a fiber bundle $M \longrightarrow B$ with fiber $F$. If the fiber and the base are aspherical spaces, then so is $M$. That is, $M, B$ and $F$ are classifying spaces for their 
fundamental groups. Then the long exact sequence of homotopy groups reduces to a short exact sequence of the form

$$
0 \longrightarrow \pi_{1}(F) \longrightarrow \pi_{1}(M) \longrightarrow \pi_{1}(B) \longrightarrow 0 .
$$

Conversely, consider the following short exact sequence

$$
0 \longrightarrow K \stackrel{i}{\longrightarrow} \Gamma \stackrel{p}{\longrightarrow} Q \longrightarrow 0 \text {. }
$$

Then there exists a fiber bundle $B \Gamma \longrightarrow B Q$ with fiber $B K$ inducing the above short exact sequence.

We can construct such a fiber bundle in the following way. For discrete groups one can consider the universal covering space $E \Gamma \longrightarrow B \Gamma$. In fact, this is the universal bundle for principal $\Gamma$-bundles. Consider the space $E \Gamma \times_{\Gamma} E Q$. Since $\Gamma$ acts freely on $E \Gamma$, this is a classifying space $B \Gamma$. Moreover, $E \Gamma / K$ is a classifying space $B K$. Therefore, by taking the quotient by $K$ first we get

$$
E \Gamma \times_{\Gamma} E Q=B K \times{ }_{Q} E Q .
$$

One can regard this space as a fiber bundle $\pi: B K \times{ }_{Q} E Q \longrightarrow B Q$ associated to the principal $Q$-bundle $E Q \longrightarrow B Q$. Furthermore, the space $E \Gamma \times_{\Gamma} E Q$ is the fiber bundle associated to the principal $Q$-bundle $E Q \longrightarrow B Q$ by $p: \Gamma \longrightarrow Q$. It is then clear that the bundle map $\pi$ induces the homomorphism $p$ at the level of fundamental groups. Thus, the long exact sequence of homotopy groups of the bundle $\pi: B K \times{ }_{Q} E Q \longrightarrow B Q$ is exactly (4.1).

We are particularly interested in central extensions $\Gamma$ of a group $Q$ by an abelian group $C$. Namely, we are interested in short exact sequences of groups of the form

$$
0 \longrightarrow C \stackrel{i}{\longrightarrow} \Gamma \stackrel{p}{\longrightarrow} Q \longrightarrow 0
$$

where $i(C)$ lies in the center of $\Gamma$.

Given a group extension as in (4.1), we derive the Lyndon-Hochschild-Serre spectral sequence as a special case of Serre spectral sequence for the associated fibration described above. In particular, the second page of the Lyndon-Hochschild-Serre spectral sequence is given by

$$
E_{2}^{p, q}=H^{p}\left(Q ; H^{q}(C ; R)\right)
$$

and it converges to the group cohomology $H^{p+q}(\Gamma ; R)$.

Remark 4.5. When $C$ is torsion of order $m$ and $R$ is $m$ divisible Example 4.4 shows that the cohomology groups $H^{r}(C ; R)$ vanish for all $r>0$. In this case the LyndonHochschild-Serre spectral sequence with values in $R$ degenerates at the second page and gives an isomorphism $H^{*}(Q ; R) \cong H^{*}(\Gamma ; R)$.

The case $C=\mathbb{Z}$ plays a special role for us. Therefore we describe this situation in further detail. When $C=\mathbb{Z}$ the fibration associated to the extension (4.2) is a principal 
$S^{1}$-bundle. Conversely, every principal $S^{1}$-bundle over a classifying space $B Q$ gives rise to a group extension by looking at the long exact sequence of homotopy groups. Moreover, the total space of such an $S^{1}$-bundle is aspherical, hence a classifying space for its fundamental group. Therefore central extensions $\Gamma$ of a group $Q$ by $\mathbb{Z}$ are classified by their Euler class of the associated principal $S^{1}$-bundle in $H^{2}(Q ; \mathbb{Z})$. We will call this the Euler class of the central extension and denote it by $e(\Gamma)$.

Remark 4.6. This is not the usual definition of the characteristic class of a central extension. In general central extensions

$$
0 \longrightarrow C \stackrel{i}{\longrightarrow} \Gamma \stackrel{p}{\longrightarrow} Q \longrightarrow 0
$$

are classified by their characteristic class in $H^{2}(Q ; C)$. Since we will not need this classification in full generality, we use our simplified definition.

Next we give a construction of the classifying space $B \pi_{1}(M)$ for a manifold $M$. In fact, we will also construct a classifying map $M \longrightarrow B \pi_{1}(M)$. Let $\Gamma$ be the fundamental group of a manifold $M$. Then a classifying space $B \Gamma$ for $\Gamma$ can be constructed in the following way. We attach cells of dimension 3 to $M$ along generators of $\pi_{2}(M)$ in order to get a space $M_{2}$ with $\pi_{2}\left(M_{2}\right)=0$. Subsequently, we attach 4-cells in order to get a space $M_{3}$ such that $\pi_{3}\left(M_{3}\right)=0$ and so on. Since we only attached cells of dimension 3 or higher, the result is an aspherical space $M_{\infty}$ which has the same fundamental group as $M$. Hence $M_{\infty}=B \Gamma$. Thus we have a natural inclusion

$$
\iota: M \longrightarrow B \Gamma \text {. }
$$

By definition $\iota$ induces an isomorphism

$$
\iota^{*}: H^{1}(B \Gamma) \longrightarrow H^{1}(M)
$$

and a injection

$$
\iota^{*}: H^{2}(B \Gamma) \longrightarrow H^{2}(M) .
$$

In particular it follows that $\mathrm{b}_{1}(\Gamma)=\mathrm{b}_{1}(M)$ for any manifold $M$ with $\pi_{1}(M)=\Gamma$.

Now suppose $\mathcal{X}$ is an orbifold. Then we can replicate the above construction on the orbifold classifying space $B \mathcal{X}$. We obtain a map $\iota: B \mathcal{X} \longrightarrow B \pi_{1}^{\text {orb }}(\mathcal{X})$ such that the homomorphisms (4.4) and (4.5) satisfy the same properties when replacing $H^{*}(M)$ by $H_{o r b}^{*}(\mathcal{X})$.

\subsection{Kähler groups}

The aim of this section is to present some properties of Kähler groups. These will serve as comparison as we discuss Sasaki groups later on. We will see in Chapter 6 that some of these properties are shared by Sasaki groups while others, arguably more 
interestingly, do not hold in Sasakian setting. If no reference is provided, the proofs of the results in this section can be found in [2].

Definition 4.7. A Kähler group is the fundamental group of a compact Kähler manifold. Analogously, the fundamental group of a smooth projective variety over $\mathbb{C}$ is a projective group. Denote by $\mathcal{K}$, respectively $\mathcal{P}$, the set of Kähler groups, resp. projective groups. We define $\mathcal{K}_{2 n}$, resp. $\mathcal{P}_{2 n}$, to be the set of fundamental groups of compact Kähler manifolds, resp. projective manifolds, of real dimension $2 n$.

Let $X$ be a $2 n$-dimensional Kähler manifold with $\pi_{1}(X)=\Gamma$. Given a finite index subgroup $\Gamma^{\prime} \subset \Gamma$, we can lift the Kähler structure to the compact covering space $X^{\prime}$ associated to $\Gamma^{\prime}$. This simple observation immediately yields the following property of Kähler groups.

Proposition 4.8. The set $\mathcal{K}_{2 n}$ is closed under taking finite index subgroups.

Clearly the product of two Kähler, respectively projective, manifolds is again Kähler, resp. projective. Thus the sets $\mathcal{P}$ and $\mathcal{K}$ are closed under taking direct products. Moreover, taking cartesian products with $\mathbb{C P}^{1}$ increases the dimension in which a Kähler or projective group can be realized. We collect these properties in a proposition for future reference.

Proposition 4.9. The classes of Kähler and projective groups enjoy the following properties.

i) The sets $\mathcal{P}$ and $\mathcal{K}$ are closed under taking direct products.

ii) There are inclusions $\mathcal{K}_{2 n} \subset \mathcal{K}_{2 n+2}$ and $\mathcal{P}_{2 n} \subset \mathcal{P}_{2 n+2}$ for all $n$.

Under a natural dimension restriction, one can prove the converse of ii) in Proposition 4.9 in the projective setting. This is due to the following version of the Lefschetz Hyperplane Theorem proven by Bott.

Theorem 4.10 (Lefschetz Hyperplane Theorem [13]). Let $X \subset \mathbb{C} P^{N}$ be a projective variety of complex dimension $n$. Consider a hyperplane section $Y \longrightarrow X$ given by $a$ hyperplane transverse to $X$. Then the induced map on homotopy groups

$$
\pi_{i}(Y) \longrightarrow \pi_{i}(X)
$$

is an isomorphism for all $i<n$.

Corollary 4.11. Every projective group is realizable in real dimension 4. In particular there are bijections $\mathcal{P}_{2 i}=\mathcal{P}_{2 j}$ for all $i, j \geqslant 2$.

Remark 4.12. Whether the same result holds for Kähler groups is still an open problem.

Remark 4.13. It follows from Kodaira's classification of complex surfaces that the set $\mathcal{P}_{4}$ coincides with the set $\mathcal{K}_{4}$. Therefore, every projective group is the fundamental group of a 4-dimensional Kähler manifold, i.e. $\mathcal{P}=\mathcal{P}_{4}=\mathcal{K}_{4}$. 
The next properties of Kähler groups that we will review are consequences of Hodge theory. In particular, the following classical result have important implications for Kähler groups.

Theorem 4.14 (Hard Lefschetz Theorem). Let $(X, \omega)$ be a compact Kähler manifold of complex dimension $n$. Then taking the wedge product with the $k$-th power of the Kähler class induces an isomorphism

$$
\begin{aligned}
L^{k}: H_{d R}^{n-k}(X) & \longrightarrow H_{d R}^{n+k}(X) \\
{[\alpha] } & \mapsto\left[\alpha \wedge \omega^{k}\right]
\end{aligned}
$$

for all $k \leqslant n$.

Moreover, Hodge theory implies that the first Betti number $b_{1}(X)$ of a Kähler manifold is even. This follows from Hodge decomposition and the fact that complex conjugation yields an isomorphism in Dolbeault cohomology. Then the construction of the inclusion (4.3) shows that $b_{1}(\Gamma)$ is even. Therefore we have the following:

Proposition 4.15. The first Betti number of a Kähler group is even.

Proposition 4.15, in turn, implies the following

Corollary 4.16. Free groups are not Kähler.

Proof. Let $F_{n}$ be the free group on $n$ generators. Then $\mathrm{b}_{1}\left(F_{n}\right)=n$. This rules out all free groups on an odd number of generators. In all other cases $F_{n}$ admits finite index subgroups which are isomorphic to $F_{N}$ for $N$ odd. Therefore the claim follows from Proposition 4.8 .

This is not the only application of Hodge theory to the study of Kähler groups. In particular, Theorem 4.14 has a non-trivial consequence which we present now. Let $\Gamma$ be the fundamental group of a compact Kähler manifold $X$ of real dimension $2 n$. Consider the isomorphism $L^{n-1}: H_{d R}^{1}(X) \longrightarrow H_{d R}^{2 n-1}(X)$. Combining this with Poincaré duality we get a non-degenerate bilinear pairing

$$
\begin{aligned}
H_{d R}^{1}(X) \times H_{d R}^{1}(X) & \longrightarrow \mathbb{R} \\
(\alpha, \beta) & \longmapsto\left\langle\alpha \cup \beta \cup \omega^{n-1},[X]\right\rangle .
\end{aligned}
$$

which factorizes through the cup product

$$
H_{d R}^{1}(X) \times H_{d R}^{1}(X) \longrightarrow H_{d R}^{2}(X) .
$$

We have seen in the previous section that there exists a map $\iota: X \longrightarrow B \Gamma$ where $\Gamma=$ $\pi_{1}(X)$. This map induces an isomorphism

$$
\iota^{*}: H^{1}(B \Gamma) \longrightarrow H^{1}(X)
$$


and a injection

$$
\iota^{*}: H^{2}(B \Gamma) \longrightarrow H^{2}(X)
$$

This is explained in the discussion before (4.4) and (4.5). Combining this with the previous discussion we get the following:

Proposition 4.17. Let $\Gamma$ be a Kähler group. Then there is a non-degenerate skewsymmetric bilinear product

$$
H^{1}(\Gamma) \times H^{1}(\Gamma) \longrightarrow \mathbb{R}
$$

which factorizes through the cup product

$$
H^{1}(\Gamma) \times H^{1}(\Gamma) \stackrel{\cup}{\longrightarrow} H^{2}(\Gamma) .
$$

Proposition 4.18. Let $\pi: M \longrightarrow \mathcal{X}$ be the principal orbibundle associated to a quasiregular Sasaki structure. Then there is a non-degenerate skew-symmetric bilinear product

$$
H^{1}\left(\pi_{1}^{o r b}(\mathcal{X}) ; \mathbb{R}\right) \times H^{1}\left(\pi_{1}^{o r b}(\mathcal{X}) ; \mathbb{R}\right) \longrightarrow \mathbb{R}
$$

which factorizes through the cup product

$$
H^{1}\left(\pi_{1}^{o r b}(\mathcal{X}) ; \mathbb{R}\right) \times H^{1}\left(\pi_{1}^{o r b}(\mathcal{X}) ; \mathbb{R}\right) \stackrel{\cup}{\longrightarrow} H^{2}\left(\pi_{1}^{o r b}(\mathcal{X}) ; \mathbb{R}\right) .
$$

Proof. Theorem 3.78 is the analogue of the Hard Leftschetz Theorem in basic cohomology of a Sasaki structure. In the quasi-regular case the basic cohomology $\operatorname{ring} H_{B}^{*}(\mathcal{F} ; \mathbb{R})$ coincides with the orbifold cohomology ring $H_{\text {orb }}^{*}(\mathcal{X} ; \mathbb{R})$. Therefore, the claim follows from the fact that the homomorphisms (4.4) and (4.5) are defined also in the orbifold case.

Example 4.19 (The Heisenberg group $\mathcal{H}_{3}$ ). Let $T^{2}=S^{1} \times S^{1}$ be the two dimensional torus. Consider the classes $\alpha_{1}, \alpha_{2} \in H^{1}\left(T^{2} ; \mathbb{Z}\right)$ given by the generators of the cohomology of the two factors. Denote by $\beta=\alpha_{1} \cup \alpha_{2}$ the generator of $H^{2}\left(T^{2} ; \mathbb{Z}\right)$. Now let $M$ be the principal $S^{1}$-bundle on the torus $T^{2}$ with Euler class $\beta$. The 3-dimensional Heisenberg group $\mathcal{H}_{3}$ is the fundamental group $\pi_{1}(M)$. Clearly $M$ is aspherical, thus $M=B \mathcal{H}_{3}$. Since $H^{2}\left(T^{2} ; \mathbb{Z}\right) \cong \mathbb{Z}$ and the Euler class is a generator, it follows from the Gysin sequence of the principal $S^{1}$-bundle that $H^{2}(M)=0$. It is then evident that the cup product of classes in $H^{1}(M)$ vanishes. Hence a non-degenerate skew-symmetric bilinear product on $H^{1}\left(\mathcal{H}_{3}\right)$ cannot factorize through the cup product. We conclude that $\mathcal{H}_{3}$ is not a Kähler group.

Example 4.20 (Higher rank Heisenberg groups). Now let $T^{2 n}$ be the $2 n$-dimensional torus and let $\alpha_{i}$ be the generator of the integral cohomology of the $i$-th factor. Denote by $\beta$ the class $\sum_{i=1}^{n} \alpha_{2 i-1} \cup \alpha_{2 i}$. Then we define the $2 n+1$-dimensional Heisenberg group $\mathcal{H}_{2 n+1}$ to be the fundamental group of the principal $S^{1}$-bundle determined by $\beta$. Carlson 
and Toledo [27] proved that $\mathcal{H}_{5}$ and $\mathcal{H}_{7}$ are not Kähler groups while Campana [20] proved that $\mathcal{H}_{2 n+1}$ is Kähler for $n \geqslant 4$.

Johnson and Rees [66] used Proposition 4.17 to show that the free product of groups with non-trivial finite quotients is not a Kähler group. In fact, they proved a much more general statement. The proof we give here is a simplified version of the original proof, relying on Lemma 4.21.

Lemma 4.21 ([78]). Let $\Gamma_{1}$ and $\Gamma_{2}$ be two groups. Assume $f_{i}: \Gamma_{i} \longrightarrow Q_{i}$ is a non-trivial quotient with kernel $K_{i}$ and $\left|Q_{i}\right|=m_{i}<\infty$ for $i=1,2$. Then the free product $\Gamma_{1} * \Gamma_{2}$ admits a finite index subgroup with odd first Betti number.

Proof. Consider the following homomorphism

$$
\Gamma_{1} * \Gamma_{2} \stackrel{\pi_{a b}}{\longrightarrow} \Gamma_{1} \times \Gamma_{2} \stackrel{f_{1} \times f_{2}}{\longrightarrow} Q_{1} \times Q_{2} .
$$

By the Kurosh subgroup theorem, the kernel of the above homomorphism has the form $F_{m} * K$ where $F_{m}$ is the free group on $m=\left(m_{1}-1\right)\left(m_{2}-1\right)$ generators and $K=K_{1} * K_{2}$. Now let $f: F_{m} \longrightarrow Q$ be a finite quotient with $|Q|=d$. Extend $f$ trivially on $K$ to get a homomorphism $\bar{f}: F_{m} * K \longrightarrow Q$. Then the kernel of $\bar{f}$ has the form $F_{n} * K * \cdots * K$ where $n=1+d(m-1)$ and $K$ appears $d$ many times. Thus, $\operatorname{ker}(\bar{f})$ is a finite index subgroup in $\Gamma_{1} * \Gamma_{2}$ and

$$
\mathrm{b}_{1}(\operatorname{ker}(\bar{f}))=n+d \mathrm{~b}_{1}(K)=1+d\left(m-1+\mathrm{b}_{1}(K)\right) .
$$

By picking $d=2 c$ we get a finite index subgroup of $\Gamma_{1} * \Gamma_{2}$ with odd first Betti number.

Theorem 4.22 ([66] $)$. Let $\Gamma_{1}$ and $\Gamma_{2}$ be groups admitting a non-trivial finite quotient. Then the group

$$
\Gamma=\left(\Gamma_{1} * \Gamma_{2}\right) \times H
$$

is not Kähler for any group $H$. In particular $\Gamma_{1} * \Gamma_{2}$ is not a Kähler group.

Proof. Suppose that the first Betti number $\mathrm{b}_{1}(H)$ of the group $H$ is even. By Lemma 4.21. there exists a finite index subgroup $\Delta \subset \Gamma_{1} * \Gamma_{2}$ with $\mathrm{b}_{1}(\Delta)$ odd. Hence, the group $\Delta \times H$ is a finite index subgroup of $\Gamma$ with odd first Betti number. Thus $\Gamma$ cannot be Kähler by Proposition 4.8 and Proposition 4.15 .

If, instead, the first Betti number $b_{1}(H)$ is odd, then $b_{1}\left(\Gamma_{1} * \Gamma_{2}\right)>0$. Thus we can assume that the first Betti number of $\Gamma_{1}$ is positive. The proof of Lemma 4.21 provides a finite index subgroup of $\Gamma_{1} \times \Gamma_{2}$ of the form $F_{n} * G$ where $F_{n}$ is the free group on $n$ generators. Moreover, the rank of $F_{n}$ can be chosen to be arbitrarily large. Since the class of Kähler groups is closed under taking finite index subgroups, we can assume $\Gamma=\left(F_{n} * G\right) \times H$ with $n>\mathrm{b}_{1}(H)$. Moreover, the bilinear product

$$
H^{1}(\Gamma) \times H^{1}(\Gamma) \longrightarrow \mathbb{R}
$$


given in Proposition 4.17 is non-degenerate and factorizes through the cup product.

Therefore $H^{1}\left(F_{n}\right)$ is an isotropic subspace in $H^{1}(\Gamma) \cong H^{1}\left(F_{n}\right) \oplus H^{1}(G) \oplus H^{1}(H)$ which is orthogonal to $H^{1}(G)$. Hence, the inequality $n>\mathrm{b}_{1}(H)$ implies that the skewsymmetric product is degenerate. Therefore $\Gamma$ cannot be a Kähler group.

Corollary 4.16 and Theorem 4.22 show that Kähler groups have little in common with free groups and free products. In [54] Gromov proved, without the technical assumption of Theorem 4.22, that Kähler groups are indecomposable under free products. These results highlight the contrast between Kähler groups and 3-manifolds groups. The class of 3-manifold groups consists of fundamental groups of (not necessarily closed) 3-manifolds. Clearly 3-manifold groups are closed under free products as the connected sum of three-manifolds is also a three-manifold. It is natural to conjecture that 3-manifolds groups have a very small intersection with Kähler groups. This was proved by Dimca and Suciu in [35] for closed 3-manifolds. Namely, they showed that fundamental groups of closed 3-manifolds are Kähler if and only if they are finite. Later Kotschick improved this result to the following:

Theorem 4.23 ([79]). An infinite Kähler group $\Gamma$ is the fundamental group of a (not necessarily closed) 3-manifold if and only if it is a surface group.

Indecomposability under free products is closely related to the number of ends of a group. Let us explain how. One can define the number of ends $E(\Gamma)$ of a group $\Gamma$ in the following way. Let $M$ be a manifold with $\pi_{1}(M)=\Gamma$ and denote by $\widetilde{M}$ the universal covering of $M$. Suppose $K_{1} \subset K_{2} \subset \cdots$ is an exhaustion of $\tilde{M}$ by compact sets. Then $E(\Gamma)$ is the limit of the number of connected components of $\widetilde{M} \backslash K_{i}$. It is a classical result that for any group $\Gamma$ the number of ends is $0,1,2$ or $\infty$. A group has 0 ends if and only if it is finite and it has 2 ends if and only if it has an infinite cyclic subgroup of finite index. By Stallings' Theorem a group has infinitely many ends if and only if it is an amalgamated product or a $\mathrm{HNN}$-estension, both over a finite group. It is then natural to ask whether a Kähler group can have infinitely many ends. The methods introduced by Gromov in [54] led to the proof (see [2, Chapter 4] or [3]) of the next theorem on ends of Kähler groups.

Theorem 4.24 ([3]). A Kähler group has 0 or 1 end.

\subsection{Analogues in the Sasakian setting}

How much do Sasaki and Kähler groups have in common? In this section we summarize the partial answers to this question which were given recently in [10, 23, 30, 68]

In order to state these recent results, we begin by giving the definition of Sasaki group.

Definition 4.25. A Sasaki group is the fundamental group of a compact Sasakian manifold. Denote by $\mathcal{S}$ the set of Sasaki groups. The set of fundamental groups of Sasakian manifolds of dimension $2 n+1$ is denoted by $\mathcal{S}_{2 n+1}$. 
One sees immediately that the Sasakian analogue of Proposition 4.8 holds true. Namely, the set $\mathcal{S}_{2 n+1}$ is closed under taking finite index subgroups. One is then encouraged to translate Proposition 4.9 in the Sasakian setting. This is where the first obstacles arise. In fact, the product of Sasakian manifolds is not Sasakian for trivial reasons. We will see in Sections 6.2 and 6.3 that Sasaki groups do not satisfy the properties in Proposition 4.9. Nevertheless, Chen [30] showed that projective groups form a subset of Sasaki groups. In that sense, these properties are satisfied by a subclass of Sasaki groups.

Theorem 4.26 ([30]). Every projective group is the fundamental group of a Sasakian manifold of dimension $2 n+1 \geqslant 7$.

Proof. Let $\Gamma=\pi_{1}(X)$ be a projective group with $X$ a smooth projective variety of complex dimension $n>1$. Let $M$ be the Boothby-Wang bundle over $X$. Then taking the join with the standard 3-sphere yields a manifold $M \star S^{3}$ diffeomorphic to an $S^{3}$ bundle over $X$. This is a Sasakian manifold with $\pi_{1}\left(M \star S^{3}\right)=\Gamma$.

In [10] the authors discussed formality of Sasakian manifolds. Their examples are also built as joins of a given Sasaki or K-contact manifold with $S^{3}$. With the same methods they proved the analogous result in the K-contact setting.

Theorem 4.27 ([10]). Every finitely presentable group is the fundamental group of a $K$-contact manifold of dimension $2 n+1 \geqslant 7$.

Proof. Given a finitely presented group $\Gamma$ there exists a symplectic $2 n$-manifold $X$ with $\pi_{1}(X)=\Gamma$ and $n>1$, see [50]. By Remark 3.23 we can construct a Botthby-Wang bundle $M$ over $X$. Now the same argument in the proof of Theorem 4.26 applies. Namely, the join $M \star S^{3}$ is a $(2 n+1)$-dimensional K-contact manifold with fundamental group $\Gamma$.

In [30] Chen proved several results on Sasaki groups which can be considered as the Sasakian analogues of results from the Kähler case. Let us review them:

Theorem 4.28. Suppose $\Gamma$ is a Sasaki group. Then $\Gamma$ is the fundamental group of some compact three-manifold $M$ if and only if $M$ has geometry modelled on $S^{3}$, the threedimensional Heisenberg group or $S \widetilde{L(2, \mathbb{R})}$.

Remark 4.29. Theorem 4.28 can be considered the Sasakian analogue of a theorem of Dimca and Suciu [35] which we discussed in Section 4.2. In Section 6.5 we prove a sharpening of this result which can be regarded as the analogue of Theorem 4.23 , see Theorem 6.21.

Theorem 4.30. Suppose $\Gamma$ is a Sasaki group.

1. Then $\Gamma$ has either zero or one end. In particular, $\Gamma$ cannot split as a non-trivial free product. 
2. If $\Gamma$ is solvable, it contains a nilpotent subgroup of finite index.

Remark 4.31. Theorem 4.30 relies on the work of Campana [21, 22] on orbifold fundamental groups of compact Kähler orbifolds, see [30, Lemma 3.1] and references therein. However, the only Kähler orbifolds that are considered in [21, 22] are those whose underlying topological space is smooth. This assumption is not always satisfied for the orbifolds associated to quasi-regular Sasaki manifolds, since these can have genuine singularities. It is not clear to the author how it is possible to apply Campana's results in this situation.

The authors of [10] discuss torsion in orbifold fundamental groups to prove the following:

Theorem 4.32 ([10]). Let $\Gamma$ be an irreducible arithmetic lattice in a semi-simple real Lie group $G$ of rank at least two with no co-compact factors and with trivial center. If $\Gamma$ is Sasaki, then it must be isomorphic to the group $\pi_{1}^{\text {orb }}(\mathcal{X})$ of some Kähler orbifold $\mathcal{X}$. Moreover, $\Gamma$ cannot be a cocompact arithmetic lattice in $S O(1, n)$ for $n \geqslant 3$.

We will see later that this is a special case of a more general statement, see Proposition 6.24 .

The results that we have seen rely only on the Structure Theorem and the join construction. On the other hand, the authors of [23] used topological properties of Sasakian manifolds to constrain their fundamental groups. Namely, in [23] is given a characterization of Sasaki nilmanifolds using the results of Tievsky [113] on minimal models for Sasakian manifolds.

Theorem 4.33 ([23]). A compact nilmanifold of dimension $2 n+1$ is Sasakian if and only if it is a quotient of the real Heisenberg group $\mathrm{H}_{2 n+1}$ by a co-compact lattice $\Gamma$.

Later Kasuya [68] extended this result to solvmanifolds. The generalization follows from the study of the representations of Sasaki groups in $G L(1, \mathbb{C})$. With the same methods Kasuya proved the following:

Theorem 4.34 ([68]). A polycyclic Sasaki group is virtually nilpotent.

We have already seen that Proposition 4.17 does not hold in the Sasakian setting. This is the first instance of a feature of Kähler groups which is not enjoyed by Sasaki groups. On the other hand, the results in this section seem to suggest that Sasaki and Kähler groups have very similar behaviour. In Chapter 6 we will see that, although Sasaki groups are deeply related to Kähler groups and enjoy many of their properties, they also display antithetical behaviour in certain aspects. 



\section{Chapter 5}

\section{Invariants and underlying structures}

Sasaki structures have a variety of underlying structures. When defining invariants of Sasaki structures it is natural to ask which of the underlying structures they depend on. In this chapter we will focus on invariants of the transverse Kähler structure. In particular, we will discuss basic Hodge numbers, basic Chern classes and the type of Sasaki structures. Moreover, we relate these invariants to the topology of the underlying almost contact and contact structures. Specifically, we will provide examples of Sasaki structures on smooth manifolds whose invariants disagree and discuss which of the underlying structures are equivalent.

In Section 5.1 we present the results in [15, 49] on invariance of basic Betti and Hodge numbers. This will serve as motivation for the results proven in Section 5.3

\subsection{Invariance of basic Betti and Hodge numbers}

In Chapter 3 we have introduced several invariants of Sasaki manifolds. In this section we will focus on basic Betti an Hodge numbers. We begin by showing that the former are topological invariants of the Sasakian manifold. In order to prove this we need a lemma of Tachibana.

Lemma 5.1 ([109]). Let $(M, \eta, \phi, R, g)$ be a compact Sasaki manifold of dimension $2 n+$ 1. Let $\alpha$ be a harmonic $p$-form on $M$ with $1 \leqslant p \leqslant n$. Then $\iota_{R} \alpha=0$ and the form $\phi \alpha$ given by

$$
\phi \alpha\left(X_{1}, \ldots, X_{p}\right)=\sum_{i=1}^{p} \alpha\left(X_{1}, \ldots, \phi X_{i}, \ldots, X_{p}\right)
$$

is harmonic.

We can now prove the following:

Theorem 5.2 ([15, Theorem 7.4.14]). Let $(M, \eta, \phi, R, g)$ be a compact Sasaki manifold of dimension $2 n+1$. Then the basic cohomology $H_{B}^{*}(\mathcal{F})$ only depends on the topology of M. In particular, the basic Betti numbers of any two Sasaki structures on M agree. 
Proof. Let $\alpha$ be a harmonic $p$-form on $M$ with $1 \leqslant p \leqslant n$. By Lemma $5.1 \iota_{R} \alpha=0$. Moreover, $\mathcal{L}_{R} \alpha=0$ because $R$ is Killing and $\alpha$ is harmonic. Hence $\alpha$ is basic. From the definition of the basic Hodge star operator $\bar{\star}$ it follows that $\star \alpha=\eta \wedge \bar{\star} \alpha$, see (3.11). This yields

$$
\mathrm{d}(\star \alpha)=\mathrm{d} \eta \wedge \bar{\star} \alpha-\eta \wedge \mathrm{d}(\bar{\star} \alpha)=L \bar{\star} \alpha-\eta \wedge \mathrm{d}_{B} \bar{\star} \alpha
$$

where $L$ is the operator defined in (3.12). Now the left-hand side vanishes since $\alpha$ is coclosed. The two forms on the right-hand side are not proportional so they both must vanish. The equation $L \bar{\star} \alpha=0$ means that $\alpha$ is primitive while the vanishing of the second term shows that $\alpha$ is basic harmonic. We conclude that $\alpha$ must be the unique harmonic representative of its class in the basic primitive cohomology group $P^{r}(\mathcal{F})$. Thus $P^{r}(\mathcal{F})$ is a topological invariant because it is isomorphic to the de Rham cohomology of $M$. Now the basic cohomology groups $H_{B}^{r}(\mathcal{F})$ are topological invariants because Theorem 3.78 gives a decomposition

$$
H_{B}^{r}(\mathcal{F})=\bigoplus_{k \geqslant 0} L^{k} P^{r-2 k}(\mathcal{F})
$$

It is natural to ask whether or not basic Hodge numbers can distinguish Sasaki structures on the same smooth manifold. This is indeed the case as shown by the following example due to Boyer and explained in [49].

Example 5.3 ([49, Example 3.4]). We present here two Sasaki structures with different Hodge numbers are given on $M=\# 21\left(S^{2} \times S^{3}\right)$, the 21-fold connected sum of $S^{2} \times S^{3}$. The first such structure $\left(\eta_{1}, \phi_{1}, R_{1}, g_{1}\right)$ is the Boothby-Wang fibration on a $K 3$ surface. Therefore, the basic Hodge numbers are the Hodge numbers of the $K 3$ surface. In particular $h^{2,0}\left(\mathcal{F}_{1}\right)=1$. On the other hand, $M$ supports the following positive Sasaki structure $\left(\eta_{2}, \phi_{2}, R_{2}, g_{2}\right)$; cf. Example 3.66 and [15, page 356]. The connected sum $\# 21\left(S^{2} \times S^{3}\right)$ can be realized as the link $L_{f}=V_{f} \bigcap S^{7} \subset \mathbb{C}^{4}$ where

$$
f\left(z_{0}, z_{1}, z_{2}, z_{3}\right)=z_{0}^{22}+z_{1}^{22}+z_{2}^{22}+z_{0} z_{3}
$$

is a weighted homogeneous polynomial of degree $d=22$ with weight $w=(1,1,1,21)$. That is, $M$ is the $S^{1}$-orbibundle over the hypersurface $X_{f} \subset \mathbb{C P}_{w}^{3}$. This Sasaki structure is positive because $\sum w_{i}-d=2>0$, see Proposition 3.85. Then a vanishing result proved independently in [95] and [51] implies that $h^{2,0}\left(\mathcal{F}_{2}\right)=0$.

It is then natural to ask whether an example of Sasaki structures of the same type but with different Hodge numbers exists, see Section 5.3 .

The fact that the Reeb foliation of a Sasaki manifold is transversally Kähler imposes some rigidity on the transverse geometry. Namely, the basic Dolbeault cohomology groups, and therefore the basic Hodge numbers, are invariant under deformations of type II, see Definition 3.51, because these deformations preserve the Reeb foliation and the transverse holomorphic structure. It turns out that basic Hodge numbers are invariant 
under an even larger class of deformations [49]. In particular they are invariant under deformations of type I.

Theorem 5.4 ([49]). Two Sasaki structures on a compact manifold with isomorphic CR structure have the same basic Hodge numbers.

The behaviour of basic Hodge numbers under general smooth deformations of Sasaki structures has been studied in [100].

\subsection{Complete intersections}

Here we present some classic results on complete intersections. These projective manifolds have a relatively simple and easily computable cohomology ring. Moreover, complete intersections often have ample canonical bundle, allowing us to construct negative Sasaki structures via Boothby-Wang fibrations, see Example 3.84.

A complete intersection $X\left(d_{1}, \ldots, d_{r}\right)$ of complex dimension $n$ and multidegree $\underline{d}=\left(d_{1}, \ldots, d_{r}\right)$ is a smooth projective variety given by the intersection of $r$ hypersurfaces of degree $d_{1}, \ldots, d_{r}$ in $\mathbb{C P}^{n+r}$. It follows from the Lefschetz Hyperplane Theorem that the cohomology of complete intersections is very simple. We give here the Dolbeault version of the theorem which was proven by Kodaira and Spencer.

Theorem 5.5 (Lefschetz Hyperplane Theorem [71]). Let $X \subset \mathbb{C} P^{N}$ be a smooth projective variety of complex dimension $n$. Consider a hyperplane section $Y \rightarrow X$ given by a hyperplane transverse to $X$. Then the induced map in Dolbeault cohomology

$$
H^{p, q}(X) \longrightarrow H^{p, q}(Y)
$$

is an isomorphism for all $p, q$ such that $p+q<n$.

Remark 5.6. This means that for $p+q<n$ we have $h^{p, q}(X)=h^{p, q}\left(\mathbb{C P}^{n}\right)$ for any complete intersection $X=X\left(d_{1}, \ldots, d_{r}\right)$. Then Serre duality implies that $h^{p, q}(X)=\delta_{p q}$ if $p+q \neq n$.

Let $X=X\left(d_{1}, \ldots, d_{r}\right)$ be a complete intersection of complex dimension $n$. Denote by $v(X)$ the normal bundle to $X$ in $\mathbb{C P}^{n+r}$. We have the following splitting

$$
T \mathbb{C P}_{\left.\right|_{X}}^{n+r}=T X \oplus v(X)=T X \oplus O\left(d_{1}\right) \oplus \cdots \oplus O\left(d_{r}\right) .
$$

Therefore, the characteristic classes of $X$ can be computed in terms of its multidegree and dimension. This computation was first carried out by Libgober and Wood in [84]. Note that the Chern and Pontryagin classes of $X$ are multiples of (the pullbacks of) powers of the generator $x \in H^{2}\left(\mathbb{C P}^{n+r}\right)$.

In order to write explicitly the characteristic classes of $X$ as multiples of $x^{i}$, denote by $d=d_{1} d_{2} \cdots d_{r}$ the total degree of $X$ and let $s_{k}=\sum_{i=1}^{r} d_{i}^{k}$. Then the coefficients of 
the Chern classes $c_{i}(X)$, Pontryagin classes $p_{i}(X)$ and the Euler characteristic $\chi(X)$ are given by polynomials in the sums $s_{k}$. Namely,

$$
\begin{aligned}
& c_{i}(X)=\frac{1}{i !} g_{i}\left(n+r+1-s_{1}, \ldots, n+r+1-s_{i}\right) x^{i}, \quad 1 \leqslant i \leqslant n, \\
& p_{i}(X)=\frac{1}{i !} g_{i}\left(n+r+1-s_{2}, \ldots, n+r+1-s_{2} k\right) x^{i}, \quad 1 \leqslant i \leqslant \frac{n}{2}, \\
& e(X)=d \frac{1}{n !} g_{n}\left(n+r+1-s_{1}, \ldots, n+r+1-s_{n}\right),
\end{aligned}
$$

where the polynomials are determined recursively by the formulas

$$
s_{i}-g_{1}\left(s_{1}\right) s_{i-1}+\frac{1}{2} g_{2}\left(s_{1}, s_{2}\right) s_{i-2}+\cdots+(-1)^{i} \frac{1}{i !} g_{i}\left(s_{1}, \ldots, s_{i}\right) i=0 .
$$

Since we are mostly interested in complete intersections of complex dimension 3 , we recall here the coefficients of the non-trivial Chern and Pontryagin classes for $n=3$.

$$
\begin{aligned}
& c_{1}=4+r-s_{1} \\
& c_{2}=\frac{1}{2}\left(\left(4+r-s_{1}\right)^{2}-\left(4+r-s_{2}\right)\right) \\
& c_{3}=\frac{1}{6}\left(\left(4+r-s_{1}\right)^{3}-3\left(4+r-s_{1}\right)\left(4+r-s_{2}\right)+2\left(4+r-s_{3}\right)\right) \\
& p_{1}=4+r-s_{2}
\end{aligned}
$$

Moreover, given the simplicity of the Hodge diamond of complete intersections, the Hirzebruch-Riemann-Roch Theorem [60] provides a method to compute the Hodge numbers of complete intersections in terms of their Chern numbers. We state here a special case which suits our discussion.

Theorem 5.7 (Hirzebruch-Riemann-Roch Theorem [60]). Let $X$ be an n-dimensional projective manifold. Let $T^{p}$ be the p-th coefficient in the Todd genus. Then

$$
\sum_{i=0}^{n}(-1)^{i} h^{i, p}=\chi^{p}(X)=T^{p}(X) .
$$

In particular $\sum_{i=0}^{n}(-1)^{i} h^{i, p}$ is a linear combination of the Chern numbers of $X$.

Summarizing the above discussion, the Hodge numbers of a complete intersection are determined by its dimension and multidegree. Moreover, the Lefschetz Hyperplane Theorem 5.5 implies

$$
\chi^{p}(X)= \begin{cases}(-1)^{n-p}\left(h^{p, n-p}+(-1)^{n}\right), & \text { if } p \neq q . \\ (-1)^{p}\left(h^{p, p}\right), & \text { if } p=\frac{n}{2}\end{cases}
$$


Since Thom noted that the diffeomorphism type of complete intersections is determined solely by the multidegree, many efforts have been made to classify complete intersections up to homemorphisms or diffeomorphisms. In particular, a result of Traving gives sufficient conditions under which the diffeomorphism type of a complete intersection is determined by the total degree $d$, the Pontryagin classes and the Euler characteristic, see [80]. Let $X\left(d_{1}, \ldots, d_{r}\right)=X(\underline{d})$ be an $n$-dimensional complete intersection. Denote by $d=\prod p^{v_{p}(d)}$ the prime factorization of $d$. Assume that $v_{p}(d) \geqslant \frac{2 n+1}{2 p-2}+1$ for all primes $p$ such that $p(p-1) \leqslant n+1$. Then we have the following:

Theorem $5.8([114])$. Let $X(\underline{d})$ and $X\left(\underline{d}^{\prime}\right)$ be two complete intersections of dimension $n>2$ satisfying the above condition. Then $X(\underline{d})$ and $X\left(\underline{d}^{\prime}\right)$ are diffeomorphic if and only if the total degrees, the Pontryagin classes and the Euler characteristic agree.

Analogously, we can define a complete intersection of codimension $r$ in a product of projective spaces $\mathbb{C P}^{n_{1}} \times \cdots \times \mathbb{C P}^{n_{k}}$ to be the intersection of hypersurfaces whose degrees are now given by $k$-tuples $\left(d_{i}^{1}, \ldots, d_{i}^{k}\right)$ whith $i=1, \ldots, r$. The computation of the Chern classes of $X$ is carried out in the same way after noticing that

$$
T\left(\mathbb{C P}^{n_{1}} \times \cdots \times \mathbb{C P}^{n_{k}}\right)_{\left.\right|_{X}}=T X \bigoplus_{i=1}^{r}\left(\otimes_{l=1}^{k} O\left(d_{i}^{l}\right)\right) .
$$

With an abuse of notation, in the equation above we are denoting by $O\left(d_{i}^{l}\right)$ the bundle $p_{l}^{*}\left(O\left(d_{i}^{l}\right)\right)$ with $p_{l}: \mathbb{C P}^{n_{1}} \times \cdots \times \mathbb{C P}^{n_{k}} \rightarrow \mathbb{C P}^{n_{l}}$ the projection on the $l$-th factor.

We will use these results in the next section to provide examples of different Sasaki structures on the same smooth manifold.

\subsection{Distinguished Sasaki structures on a smooth manifold}

In this section we construct examples of Sasaki structures on the same smooth manifold distinguished by transverse invariants. These examples are given by Boothby-Wang fibrations over smooth projective varieties. The invariants that we use to distinguish the Sasaki structures are basic Hodge numbers, divisibility of the basic first Chern class and the type of the Sasaki structure. In particular, we give examples of negative Sasaki structures with different Hodge numbers in all dimensions $2 n+1 \geqslant 5$.

\subsubsection{Simply connected 5-manifold}

Firstly we focus on 5-dimensional Sasaki structures. In particular we will consider simply connected regular Sasaki manifolds. In this setting, that is, regular contact structures on simply connected 5-manifolds with indivisible Euler class, the equivalence classes of (almost) contact structured were studied by Hamilton [59]. Let us introduce the terminology needed in order to state his result. 
Definition 5.9. Let $X$ be a topological space and pick $\alpha \in H^{p}(X ; \mathbb{Z})$. The divisibility $d(\alpha)$ of a class $\alpha$ is the maximum number $n \in \mathbb{Z}$ such that $\alpha=n \beta$ for some $0 \neq \beta \in$ $H^{p}(X ; \mathbb{Z})$. A class $\alpha$ is called indivisible, or primitive, if $d(\alpha)=1$.

Given a regular K-contact manifold $\pi: M \longrightarrow X$, let $\mathcal{D}$ be the underlying almost contact structure. Denote by $c_{1}(\mathcal{D})$ the first Chern class of $\mathcal{D}$ and by $c_{1}(X)$ the first Chern class of the symplectic manifold $X$. We will be interested in the divisibility of these classes in $H^{2}(M)$ and $H^{2}(X)$ respectively.

We are now ready to state following result of Hamilton:

Theorem 5.10 ([59]). Let $M$ be a simply connected 5-manifold admitting two different (regular) Boothby-Wang fibrations

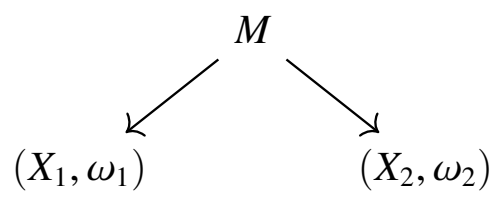

and denote by $\left(\eta_{i}, \phi_{i}, R_{i}, g_{i}\right)$ the associated $K$-contact structures for $i=1,2$.

1. Then the underlying almost contact structures are equivalent if and only if the divisibilities of their first Chern classes agree, i.e. $d\left(c_{1}\left(\mathcal{D}_{1}\right)\right)=d\left(c_{1}\left(\mathcal{D}_{2}\right)\right)$ in $H^{2}(M)$.

2. Assume that the underlying contact structures are equivalent.

- If $d\left(c_{1}\left(\mathcal{D}_{1}\right)\right)=d\left(c_{1}\left(\mathcal{D}_{2}\right)\right)=0$ in $H^{2}(M)$, then $d\left(c_{1}\left(X_{1}\right)\right)=d\left(c_{1}\left(X_{2}\right)\right)$ in $H^{2}\left(X_{i}\right)$.

- If $d\left(c_{1}\left(\mathcal{D}_{1}\right)\right)=d\left(c_{1}\left(\mathcal{D}_{2}\right)\right) \neq 0$ in $H^{2}(M)$, then either $d\left(c_{1}\left(X_{1}\right)\right), d\left(c_{1}\left(X_{2}\right)\right) \leqslant$ 3 or $d\left(c_{1}\left(X_{1}\right)\right)=d\left(c_{1}\left(X_{2}\right)\right) \geqslant 4$ in $H^{2}\left(X_{i}\right)$.

As discussed in the introduction the contact structures underlying Sasaki structures are tight. Hence they are not necessarily classified by an $h$-principle, see [12]. In particular, the isotopy classes of contact structures underlying Sasaki structures can lie in the same homotopy class of almost contact structures. In general homotopy classes of almost contact structures are determined by obstruction theory. By these means Geiges [47] proved that almost contact structures on simply connected 5-manifolds are classified up to homtopy by their first Chern class.

Theorem 5.11 ([47]). Let $M$ be a simply connected 5-manifold. Then two almost contact structures on $M$ are homotopic if and only if they have the same first Chern class.

Remark 5.12. Let $\pi: M \longrightarrow X$ be a regular Sasaki structure. Then the first Chern class $c_{1}(\mathcal{D})$ of the contact distribution $\mathcal{D}$ is the pullback $\pi^{*}\left(c_{1}(X)\right)$ of first Chern class of the base $X$. 
The previous remark also gives a necessary and sufficient condition for $M$ to be spin which only depends on $X$ :

Lemma 5.13. Let $M$ be a Boothby-Wang bundle over a smooth projective manifold $(X, \omega)$. Then $M$ is spin if and only if $X$ is spin or $c_{1}(X) \equiv[\omega] \bmod 2$.

Proof. Denote by $\mathcal{D}$ and $L_{R}$ the contact distribution and the Reeb line bundle given by the Boothby-Wang structure on $M$. Since $T M=\mathcal{D} \oplus L_{R}$ the Whitney sum formula gives $w_{2}(M)=w_{2}(\mathcal{D})$. Moreover, $w_{2}(\mathcal{D})$ is the mod 2 reduction of $c_{1}(\mathcal{D})$. It follows from Remark 5.12 that $w_{2}(M)=\pi^{*}\left(w_{2}(X)\right)$. Therefore $w_{2}(M)=0$ if and only if $w_{2}(X)=0$ or $w_{2}(X) \in \operatorname{ker} \pi^{*}$, that is, $c_{1}(X) \equiv[\omega] \bmod 2$.

Remark 5.14. Suppose $M$ is a simply connected 5-manifold with torsion-free cohomology. Then Barden's classification of simply connected 5-manifolds [7] implies that the diffeomorphism type of $M$ depends only on its second Betti number and whether $M$ is spin or non-spin. Namely,

$$
M \cong \begin{cases}\# \mathrm{~b}_{2}(M)\left(S^{2} \times S^{3}\right), & \text { if } w_{2}(M)=0 . \\ \#\left(\mathrm{~b}_{2}(M)-1\right)\left(S^{2} \times S^{3}\right) \#\left(S^{2} \widetilde{\times} S^{3}\right), & \text { if } w_{2}(M) \neq 0 .\end{cases}
$$

where $S^{2} \widetilde{\times} S^{3}$ is the non-trivial $S^{3}$-bundle over $S^{2}$.

Lemma 5.15. Let $X_{1}$ and $X_{2}$ be simply connected Kähler surfaces endowed with indivisible integral Kähler classes $\left[\omega_{1}\right]$ and $\left[\omega_{2}\right]$ respectively. Suppose that $b_{2}\left(X_{1}\right)=b_{2}\left(X_{2}\right)$. Then the associated Boothby-Wang fibrations $M_{1}$ and $M_{2}$ are diffeomorphic if and only if they are both spin or non-spin, i.e. if and only if $w_{2}\left(M_{1}\right)$ and $w_{2}\left(M_{2}\right)$ have the same parity.

Proof. Since the Kähler class $\left[\omega_{i}\right]$ is indivisible, the Boothby-Wang bundle $M_{i}$ is simply connected with torsion-free cohomology for $i=1,2$. Moreover $\mathrm{b}_{2}\left(M_{i}\right)=\mathrm{b}_{2}\left(X_{i}\right)-1$. Thus the claim is a direct consequence of Remark 5.14 .

Lemma 5.15 is the key observation to construct most examples of diffeomorphic 5dimensional Boothby-Wang bundles in this section. Before presenting some results on simply connected Sasakian manifolds in dimension 5 we a state a lemma about complex surfaces for future reference.

Lemma 5.16. Let $X$ be a simply connected complex surface. Then its Hodge numbers $h^{0,2}$ and $h^{1,1}$ are related to its Chern numbers $c_{1}^{2}$ and $c_{2}$ by the following formulas:

$$
\begin{aligned}
h^{0,2} & =\frac{1}{12}\left(c_{1}^{2}+c_{2}\right)-1 \\
h^{1,1} & =\frac{1}{6}\left(5 c_{2}-c_{1}^{2}\right) .
\end{aligned}
$$


We now give a result on Sasaki structures with inequivalent underlying contact structures and different basic Hodge numbers on a simply connected 5-manifold.

Proposition 5.17. Let $X_{1}$ and $X_{2}$ be simply connected complex surfaces with ample canonical classes $K_{X_{1}}$ and $K_{X_{2}}$ respectively. Suppose that $b_{2}\left(X_{1}\right)=b_{2}\left(X_{2}\right)$. Then the principal $S^{1}$-bundle $M$ over $X_{1}$ with Euler class the indivisible class underlying $K_{X_{1}}$ admits two negative Sasaki structures with homotopic underlying almost contact structures. Moreover,

i) if $\sigma\left(X_{1}\right) \neq \sigma\left(X_{2}\right)$, then the Sasaki structures have different basic Hodge numbers,

ii) if $\operatorname{gcd}\left(K_{X_{1}}^{2}, K_{X_{2}}^{2}\right)$ is square-free and $d\left(K_{X_{1}}\right) \neq 1$ or $d\left(K_{X_{2}}\right) \neq 1$, then the underlying contact structures are inequivalent.

Proof. By the Kodaira Embedding Theorem the indivisible classes underlying $K_{X_{1}}$ and $K_{X_{2}}$ are represented by Kähler forms. The associated Boothby-Wang bundles $M_{1}$ and $M_{2}$ are both spin because $K_{X_{i}}=-c_{1}\left(X_{i}\right)$ is a multiple of the Euler class, hence $\pi^{*}\left(c_{1}\left(X_{i}\right)\right)=$ 0 ; cf. Remark 5.12. Moreover, $\mathrm{b}_{2}\left(M_{1}\right)=\mathrm{b}_{2}\left(M_{2}\right)$ because the Euler classes are indivisible. Therefore, $M_{1}$ is diffeomorphic to $M_{2}$ by Lemma 5.15. In addition the Sasaki structures are negative because $c_{1}\left(X_{i}\right)$ is a negative multiple of a Kähler class. The underlying almost contact structures are homotopic as a consequence of Theorem 5.11 because $c_{1}\left(\mathcal{D}_{i}\right)=\pi^{*}\left(c_{1}\left(X_{i}\right)\right)=0$.

To prove part i) notice first that the basic Hodge numbers of $M_{i}$ are the Hodge numbers of $X_{i}$ for $i=1,2$. Since $\sigma\left(X_{1}\right) \neq \sigma\left(X_{2}\right)$, we can assume $\mathrm{b}_{2}^{+}\left(X_{1}\right)=\mathrm{b}_{2}^{+}\left(X_{2}\right)+a$ for some $a>0$. Now on a simply connected complex surface we have

$$
c_{1}^{2}=2 c_{2}+p_{1}=2\left(2+b_{2}^{+}+b_{2}^{-}\right)+3\left(b_{2}^{+}-b_{2}^{-}\right)=4+5 b_{2}^{+}-b_{2}^{-} .
$$

Thus $\mathrm{b}_{2}^{+}\left(X_{1}\right)=\mathrm{b}_{2}^{+}\left(X_{2}\right)+a$ implies $c_{1}^{2}\left(X_{1}\right)=c_{1}^{2}\left(X_{2}\right)+7 a$. On the other hand $c_{2}\left(X_{i}\right)=$ $\mathrm{b}_{2}\left(X_{i}\right)+2$. Therefore $c_{2}\left(X_{1}\right)=c_{2}\left(X_{2}\right)$. Now, by Lemma 5.16, the Hodge numbers are related to the Chern numbers by

$$
\frac{1}{12}\left(c_{1}^{2}\left(X_{i}\right)+c_{2}\left(X_{i}\right)\right)=\chi\left(O_{X_{i}}\right)=h^{2,0}\left(X_{i}\right)+1
$$

because $\mathrm{b}_{1}\left(X_{i}\right)=0$. Therefore the basic Hodge numbers of $M_{1}$ and $M_{2}$ disagree if $a \neq 0$.

Now, without loss of generality, suppose that $d\left(K_{X_{1}}\right) \neq 1$ and $\operatorname{gcd}\left(K_{X_{1}}^{2}, K_{X_{2}}^{2}\right)$ is square-free. If the contact structures were equivalent, then $d\left(c_{1}\left(X_{1}\right)\right)=d\left(c_{1}\left(X_{2}\right)\right)$ in $H^{2}\left(X_{i}\right)$ by Theorem 5.10. This means that $c_{1}\left(X_{i}\right)=k \cdot \alpha_{i}$ for some primitive class $\alpha_{i} \in H^{2}\left(X_{i}\right)$ and some integer $k>1$. Therefore $K_{X_{i}}^{2}=c_{1}^{2}\left(X_{i}\right)=k^{2} \cdot \alpha_{i}^{2}$ contradicting the hypothesis. This proves part ii).

A first application of the results above is the following: 
Theorem 5.18. There exist countably infinitely many simply connected 5-dimensional manifolds admitting negative Sasaki structures with inequivalent underlying contact structures in the same homotopy class of almost contact structures.

Proof. The proof makes use of a family of complete intersections presented in [18, Example 4]

Let $X_{k}$ be the hypersurface of bidegree $(5+k, 6)$ in $\mathbb{C P}^{1} \times \mathbb{C P}^{2}$. As explained in Section 5.2, the computation of the characteristic numbers of $X_{k}$ is carried out by noticing that $T\left(\mathbb{C P}^{1} \times \mathbb{C P}^{2}\right)_{\mid X_{k}}=v\left(X_{k}\right) \oplus T X_{k}$. In particular we have $c_{1}\left(X_{k}\right)=-(k+3) x_{1}-3 x_{2}$ where $x_{1}$ and $x_{2}$ are the generators of the cohomology rings of $\mathbb{C P}$ and $\mathbb{C P}{ }^{2}$ respectively.

Consider now the complete intersection $Y_{k}$ of multidegree $[(2,1),(1+k, 6)]$ in $\mathbb{C P}^{1} \times$ $\mathbb{C P}^{3}$. We can compute the characteristic numbers of $Y_{k}$ as above. In this case we have $c_{1}\left(Y_{k}\right)=-(k+1) y_{1}-3 y_{2}$, where $y_{1}$ and $y_{2}$ are now the generators of the cohomology rings of $\mathbb{C P} P^{1}$ and $\mathbb{C P}^{3}$ respectively. The calculation of the characteristic numbers yields

$$
c_{1}^{2}\left(X_{k}\right)=c_{1}^{2}\left(Y_{k}\right)=9(17+5 k), c_{2}\left(X_{k}\right)=c_{2}\left(Y_{k}\right)=3(113+25 k) .
$$

Thus $\mathrm{b}_{2}\left(X_{k}\right)=\mathrm{b}_{2}\left(Y_{k}\right)=337+75 k$. Since the canonical bundles $K_{X_{k}}$ and $K_{Y_{k}}$ are ample, the Kodaira Embedding Theorem yields a Kähler form representing the indivisible classes underlying $K_{X_{k}}$ and $K_{Y_{k}}$. Hence we can perform the Boothby-Wang construction with Euler classes given by such Kähler classes. The resulting 5-manifolds $M_{X}$ and $M_{Y}$ are spin because the first Chern class $c_{1}\left(X_{k}\right)$, respectively $c_{1}\left(Y_{k}\right)$, is a multiple of the Euler class of the bundle. Hence, $M_{X}$ and $M_{Y}$ are diffeomorphic because they have torsion-free cohomology and they have the same second Betti number, see Proposition 5.17 .

These two Sasaki structures cannot be distinguished by the basic Hodge numbers because the characteristic numbers of $X_{k}$ and $Y_{k}$ agree, see Lemma 5.16. Moreover, since the first Chern classes $c_{1}\left(X_{k}\right)$ and $c_{1}\left(Y_{K}\right)$ are in the kernel of the pullback, the underlying almost contact structures are homotopic by Theorem 5.11. Nevertheless, by the above computation of $c_{1}\left(X_{k}\right)$ and $c_{1}\left(Y_{K}\right)$, the divisibilities of the first Chern classes are

$$
d\left(c_{1}\left(X_{k}\right)\right)=\operatorname{gcd}(k, 3), \quad d\left(c_{1}\left(Y_{K}\right)\right)=\operatorname{gcd}(k+1,3) .
$$

Thus the underlying contact structures are inequivalent for $k \neq 3 l+1$ by Theorem 5.10 . We conclude that the two Sasaki structures cannot be equivalent unless $k=3 l+1$.

Remark 5.19. The above construction relies on examples of pairs of homeomorphic complete intersections of complex dimension 2 whose canonical classes have different divisibilities. Any pair of such complete intersections provides two Sasaki structures on the same smooth manifold with the property that the underlying almost contact structures are equivalent while the contact structures are not. Further examples of such pairs can be found in [38, Theorem 5] and [117, Table 1].

When looking at Theorem 5.18 it is natural to ask whether or not there is a bound on the number of Sasaki structures with inequivalent contact structures but homotopic 
almost contact structures. The following proposition gives a negative answer to this question.

Theorem 5.20. For all positive integers $k>0$ there exists a simply connected 5manifold admitting $k$ Sasaki structures with homotopic almost contact structures but pairwise inequivalent contact structures.

Proof. In [18] Braungardt and Kotschick constructed arbitrarily large tuples $\left(X_{1}, \ldots, X_{k}\right)$ of homeomorphic branched covers of projective planes $\mathbb{C P}^{2}$. These are pairwise nondiffeomorphic projective surfaces distinguished by the divisibility of the first Chern class. Moreover, the surfaces $X_{i}$ have ample canonical bundle $K_{X_{i}}$ for $i=1, \ldots, k$; cf. [18, Corollary 1].

By Proposition 5.17 we can perform the Boothby-Wang construction to get $k$ Sasaki structures on a simply connected spin 5-manifold. Since the first Chern class $c_{1}\left(X_{i}\right)$ is a multiple of the Euler class, the underlying almost contact structures are homotopic by Theorem 5.11. Moreover, the equalities

$$
\chi=c_{2}, \quad \sigma=\frac{1}{3} p_{1}=\frac{1}{3}\left(c_{1}^{2}-2 c_{2}\right)
$$

together with Lemma 5.16 show that the Hodge numbers of complex surfaces are topological invariants. Hence the basic Hodge numbers of the Boothby-Wang fibrations agree.

However, the basic first Chern classes $c_{1}\left(X_{i}\right)$ have pairwise different divisibilities. Thus the underlying contact structures are inequivalent by Theorem 5.10 .

Next we turn our attention to the relation between basic Hodge numbers, the type and homotopy classes of underlying almost contact structures. Namely, we show that a manifold can support two Sasaki structures with different Hodge numbers even if they are both negative and the underlying almost contact structures are homotopic.

Theorem 5.21. There exist countably infinitely many simply connected 5-manifolds admitting two negative Sasaki structures whose basic Hodge numbers disagree. Moreover, these pairs of Sasaki structures have homotopic underlying almost contact structures but inequivalent contact structures.

Proof. We construct these Sasaki manifolds as Boothby-Wang fibrations over a family of complete intersections and a family of Horikawa surfaces respectively.

Let $X_{k}$ be the complete intersection in $\mathbb{C P}^{1} \times \mathbb{C P}^{3}$ given by intersecting hypersurfaces of bidegree $(2,5)$ and $(k, 1)$. As explained in Section 5.2, the computation of the characteristic numbers of $X_{k}$ is carried out by noticing that $T\left(\mathbb{C P}^{1} \times \mathbb{C P}^{3}\right)_{\mid X_{k}}=v\left(X_{k}\right) \oplus T X_{k}$. In particular, we have $c_{1}\left(X_{k}\right)=-k x_{1}-2 x_{2}$ where $x_{1}$ and $x_{2}$ are the generators of the cohomology rings of $\mathbb{C P}^{1}$ and $\mathbb{C P}^{3}$ respectively. The Chern numbers and holomorphic Euler characteristic of $X_{k}$ are:

$$
c_{1}^{2}\left(X_{k}\right)=40 k+8, \quad c_{2}\left(X_{k}\right)=80 k+76, \quad \chi\left(O_{X_{k}}\right)=10 k+7 .
$$


Moreover, $X_{k}$ is simply connected thus the second Betti number is given by $b_{2}\left(X_{k}\right)=$ $c_{2}\left(X_{k}\right)-2=80 k+74$.

On the other hand, consider the following family of Horikawa surfaces $Y_{i}$ from [61]. Let $\Sigma_{i}$ be the Hirzebruch surface of degree $i$, that is the $\mathbb{C P}{ }^{1}$-bundle over $\mathbb{C P}^{1}$ whose zero section $\Delta$ has self-intersection $-i$. Let $F$ denote the class of the fiber of the fibration. Then we can construct the Horikawa surface $Y_{i}$ as the double cover $p r: Y_{i} \rightarrow \Sigma_{i}$ with branch locus homologous to $B=6 \Delta+2(2 i+3) F$. Notice that these surfaces have ample canonical bundle $K_{Y_{i}}$ since $K_{Y_{i}}=p r^{*}\left(K_{\Sigma_{i}}+\frac{1}{2} B\right)=p r^{*}(\Delta+(i+1) F)$ and $\Delta+(i+1) F$ is an ample bundle. Moreover, these surfaces are simply connected because the branch locus $B$ is ample. Now the characteristic numbers of $Y_{i}$ are:

$$
c_{1}^{2}\left(Y_{i}\right)=2 i+4, \quad c_{2}\left(Y_{i}\right)=10 i+56, \quad \chi\left(O_{Y_{i}}\right)=i+5 .
$$

Hence $b_{2}\left(Y_{i}\right)=10 i+54$ so $b_{2}\left(X_{k}\right)=b_{2}\left(Y_{i}\right)$ for $i=8 k+2$.

From now on we denote $Y_{8 k+2}$ by $Y_{k}$ and restrict to this case for which we have:

$$
c_{1}^{2}\left(Y_{k}\right)=16 k+8, \quad c_{2}\left(Y_{k}\right)=80 k+76, \quad \chi\left(O_{Y_{k}}\right)=8 k+7 .
$$

Both $X_{k}$ and $Y_{k}$ have ample canonical line bundle. Hence, by the Kodaira Embedding Theorem, we can perform the Boothby-Wang construction with indivisible Euler class underlying $K_{X_{k}}$, respectively $K_{Y_{k}}$, see Proposition 5.17. Denote the associated Boothby-Wang fibration over $X_{k}$, respectively over $Y_{k}$, by $\left(M_{1}, \eta_{1}, \phi_{1}, R_{1}, g_{1}\right)$, resp. $\left(M_{2}, \eta_{2}, \phi_{2}, R_{2}, g_{2}\right)$. Since the canonical classes $K_{X_{k}}$ and $K_{Y_{k}}$ are multiples of the respective Kähler classes, the associated Sasaki manifolds $M_{1}$ and $M_{2}$ are spin. Hence $M_{1}$ and $M_{2}$ are both diffeomorphic to the $(80 k+73)$-fold connected sum \# $(80 k+73)\left(S^{2} \times S^{3}\right)$ by Lemma 5.15. Since the Sasaki structures are regular, the basic Hodge numbers are the Hodge numbers of the base of the Boothby-Wang fibration. Therefore Lemma 5.16 gives

$$
\begin{array}{cc}
h^{0,2}\left(X_{k}\right)=10 k+6, & h^{1,1}\left(X_{k}\right)=60 k+62, \\
h^{0,2}\left(Y_{k}\right)=8 k+6, & h^{1,1}\left(Y_{k}\right)=64 k+62 .
\end{array}
$$

Moreover, the underlying almost contact structures are homotopic by Proposition 5.17 .

Notice that $\mathrm{d}\left(c_{1}\left(X_{k}\right)\right)=\operatorname{gcd}\{k, 2\}$. On the other hand, the main result of [91] implies that $Y_{k}$ is spin if and only if $B / 2$ is the Poincare dual of the second Stiefel-Whitney class $w_{2}\left(\Sigma_{8 k+2}\right)$. In other words, $Y_{k}$ is spin if and only if $B / 2$ is divisible by 2 because $\Sigma_{8 k+2}$ is spin. Now the intersection number of $B / 2$ with $F$ equals 3 and this implies that $Y_{k}$ is not spin. In particular, since $\mathrm{d}\left(K_{Y}\right)$ is always odd, the structures are not equivalent as contact structures whenever $k$ is even.

Note that the Sasaki structures we have constructed in Theorem 5.18, Theorem 5.20 and Theorem 5.21 are negative. On the other hand, the two Sasaki structures in Exam- 
ple 5.3 are positive and null. This is the first example of a manifold admitting Sasaki structures of the same type whose basic Hodge numbers disagree.

On the other hand, the next result shows that one can have structures of different type whose underlying almost contact structures are homotopic.

Proposition 5.22. There exists a simply connected 5-manifold admitting negative, positive and null Sasaki structures which have homotopic almost contact structures.

Proof. Let us denote by $X$ the del Pezzo surface $\mathbb{C P}^{2} \# 8 \overline{\mathbb{C P}^{2}}$. This is a complex surface with ample anti-canonical class $-K_{X}$ represented by a Kähler form.

On the other hand consider the Craighero-Gattazzo surface $Y$, see [32]. It was proven in [37] that the Craighero-Gattazzo surface $Y$ has ample canonical class $K_{Y}$. This can therefore be represented by a Kähler form. Moreover, $Y$ is simply connected [99].

Thus $X$ and $Y$ are homeomorphic by Freedman's classification. Denote by $M_{X}$ and $M_{Y}$ the Boothby-Wang bundles over $X$ and $Y$ respectively. The former is positive while the latter is negative because $c_{1}(X)$, respectively $c_{1}(Y)$, is a positive, resp. negative, multiple of the Euler class $[\omega]$.

Both $M_{X}$ and $M_{Y}$ are diffeomorphic to $\# 8\left(S^{2} \times S^{3}\right)$ by Barden's classification of simply connected 5-manifolds. Moreover, $\# 8\left(S^{2} \times S^{3}\right)$ admits several null structures as a link, see [15, Table B.1]. For instance, the Boothby-Wang bundle over a hypersurface of degree $d=17$ in $\mathbb{C P}(2,3,5,7)$ is a null Sasaki manifold diffeomorphic to $\# 8\left(S^{2} \times S^{3}\right)$.

In all cases the first Chern class of the contact distribution vanishes. Hence the underlying almost contact structures are homotopic by Theorem 5.11 .

Remark 5.23. In the notation of Proposition 5.22 $K_{X}^{2}=K_{Y}^{2}=1$, therefore the first basic Chern classes are indivisible. It is not clear whether or not the contact structures are isotopic. Moreover, the Hodge numbers of $X$ and $Y$ agree since they are topological invariants of complex surfaces. Hence the Sasaki structures cannot be distinguished by their basic Hodge numbers.

\subsubsection{Simply connected 7-manifolds}

Here we turn our attention to Sasaki structures on 7-dimensional manifolds. In this setting we cannot rely on an analogue to Theorem 5.10 to classify equivalent contact structures, nor there exists a classification of almost contact structures on 7-manifolds. Nevertheless, when restricting to Boothby-Wang fibrations over complete intersections one has some control on invariants such as the basic Hodge numbers.

Let us begin with a discussion on complete intersections of complex dimension 3 . Let $X$ be such a complete intersection. As seen in Remark 5.6 the interesting Hodge 
numbers $h^{p, q}$ lie in the middle degree. That is, its Hodge diamond is given by

$$
\begin{aligned}
& 1 \\
& 0 \quad 0 \\
& \begin{array}{lll}
0 & 1 & 0
\end{array} \\
& h^{3,0} \quad h^{2,1} \quad h^{1,2} \quad h^{0,3} \\
& \begin{array}{lll}
0 & 1 & 0
\end{array} \\
& 0 \quad 0 \\
& 1
\end{aligned}
$$

as showed in the discussion leading to (5.6).

We are particularly interested in diffeomorphic complete intersections with different Hodge numbers. By the Hirzebruch-Riemann-Roch Theorem 5.7 the middle dimensional Hodge numbers of a 3-dimensional complete intersection $X$ can be computed from the Chern numbers:

$$
\begin{aligned}
& h^{0,3}=h^{3,0}=1-\chi^{0}(X)=1-\frac{1}{24} c_{1} c_{2} \\
& h^{1,2}=h^{2,1}=1+\chi^{1}(X)=1+\frac{1}{24} c_{1} c_{2}-\frac{1}{2} c_{3} .
\end{aligned}
$$

The following lemma characterizes diffeomorphic complete intersections with different Hodge numbers in terms of the first Chern class.

Lemma 5.24. Two diffeomorphic complete intersection $X$ and $Y$ of complex dimension 3 have different Hodge numbers if and only if $c_{1}(X) \neq c_{1}(Y)$.

Proof. Since $X$ and $Y$ are diffeomorphic, we have $p_{1}(X)=p_{1}(Y)$ and $c_{3}(X)=c_{3}(Y)$. It is clear that, if $c_{1}(X)=c_{1}(Y)$, then also $c_{2}(X)=c_{2}(Y)$ because $p_{1}=c_{1}^{2}-2 c_{2}$. Thus all the Chern numbers agree. It follows from (5.8) and (5.9) that the Hodge numbers of $X$ and $Y$ agree. Conversely, denote by $x$ the generator of the second cohomology group determined by the orientation. Suppose

$$
\begin{aligned}
& c_{1}(X)=k \cdot x \\
& c_{1}(Y)=l \cdot x \\
& p_{1}(X)=p_{1}(Y)=m \cdot x^{2}
\end{aligned}
$$


with $|k|>|l|$. Then we have

$$
\begin{aligned}
& 2 c_{2}(X)=c_{1}(X)^{2}-p_{1}(X)=\left(k^{2}-m\right) \cdot x^{2} \\
& 2 c_{2}(Y)=c_{1}(Y)^{2}-p_{1}(Y)=\left(l^{2}-m\right) \cdot x^{2} .
\end{aligned}
$$

Thus $c_{2}(X) \neq c_{2}(Y)$ since $k^{2}-m>l^{2}-m$. This implies that

$$
\begin{aligned}
& 2 c_{1}(X) c_{2}(X)=k\left(k^{2}-m\right) \cdot x^{3} \\
& 2 c_{1}(Y) c_{2}(Y)=l\left(l^{2}-m\right) \cdot x^{3} .
\end{aligned}
$$

Therefore the equalities (5.8) and (5.9) yield

$$
\begin{aligned}
& h^{0,3}(X)>h^{0,3}(Y) \\
& h^{1,2}(X)<h^{1,2}(Y) .
\end{aligned}
$$

Complete intersections of complex dimension 3 are simply connected 6-manifolds with torsion-free cohomology. Therefore, by Wall's classification the diffeomorphism type of 3-dimensional complete intersection is determined by the Euler number, the first Pontryagin class and the parity of the first Chern class, see [67, 116]. In their study of moduli spaces of complete intersections Libgober and Wood [84] conjectured the existence of diffeomorphic complete intersections with different Chern classes. Such pairs of complete intersections $\left(X_{1}, X_{2}\right)$ were given by Wang and Du [117]:

\begin{tabular}{c|cccc}
$\underline{d}$ & $d$ & $p_{1}$ & $\chi=d \cdot c_{3}$ & $c_{1}$ \\
\hline$(70,16,16,14,7,6)$ & $7^{3} \cdot 5 \cdot 3 \cdot 2^{11}$ & -5683 & -7767425433600 & -119 \\
$(56,49,8,6,5,4,4)$ & $7^{3} \cdot 5 \cdot 3 \cdot 2^{11}$ & -5683 & -7767425433600 & -121 \\
\hline$(88,28,19,14,6,6)$ & $19 \cdot 11 \cdot 7^{2} \cdot 3^{2} \cdot 2^{8}$ & -9147 & -35445749391360 & -151 \\
$(76,56,11,7,6,6,2)$ & $19 \cdot 11 \cdot 7^{2} \cdot 3^{2} \cdot 2^{8}$ & -9147 & -35445749391360 & -153 \\
\hline$(84,29,25,25,18,7)$ & $29 \cdot 7^{2} \cdot 5^{4} \cdot 3^{3} \cdot 2^{3}$ & -9510 & -384536710530000 & -178 \\
$(60,58,49,9,5,5,5)$ & $29 \cdot 7^{2} \cdot 5^{4} \cdot 3^{3} \cdot 2^{3}$ & -9510 & -384536710530000 & -180 \\
\hline
\end{tabular}

Table 5.1: Diffeomorphic 3-dimensional complete intersections with different $c_{1}$

The characteristic classes of a complete intersection $X$ are multiples of the generators $x, x^{2}$ of the groups $H^{2}(X) \cong H^{4}(X) \cong \mathbb{Z}$. The values in Table 5.1 are the coefficients that determine the characteristic classes as multiples of $x$ and $x^{2}$.

These pairs of complete intersections allows us to extend Theorem 5.21 to dimension 7 although in the following weaker form:

Theorem 5.25. There exist countably many 7-manifolds admitting two negative Sasaki 
structures with different basic Hodge numbers. Moreover, these manifolds can be arranged to be spin or non-spin.

Proof. Let $X_{1}$ and $X_{2}$ be two diffeomorphic 3-dimensional complete intersections with different first Chern class given in Table 5.1. Since these complete intersections have ample canonical bundle, the positive generator $x$ of $H^{2}\left(X_{1}\right) \cong H^{2}\left(X_{2}\right)$ is a Kähler class by Kodaira Embedding Theorem. Thus we can perform the Boothby-Wang construction with Euler class $x$, see Proposition 5.17 .

The resulting Sasaki manifolds $\left(M, \eta_{1}, \phi_{1}, R_{1}, g_{1}\right)$ and $\left(M, \eta_{2}, \phi_{2}, R_{2}, g_{2}\right)$ are diffeomorphic because $X_{1}$ and $X_{2}$ are diffeomorphic and the Euler classes of the BoothbyWang bundles coincide. Moreover, $H^{2}(M)=0$ because $H^{2}\left(X_{1}\right)$ is generated by the Euler class $x$. Then Lemma 5.24 and Table 5.1 imply that these structures are distinguished by their basic Hodge numbers which coincide with the Hodge numbers of $X_{1}$ and $X_{2}$.

Now let $X_{1}$ and $X_{2}$ be two diffeomorphic 3-dimensional complete intersections with odd first Chern class given in Table 5.1. Let $M_{i}^{k}$ be the Boothby-Wang bundle over $X_{i}$ with Euler class $k \cdot x$. We can arrange the manifold $M_{i}^{k}$ to be spin or non-spin. In fact this depends on the parity of $k$. For $k$ odd the second homology group $H^{2}\left(M_{i}^{k} ; \mathbb{Z}\right) \cong$ $H^{2}\left(X_{i} ; \mathbb{Z}\right) / k x \cong \mathbb{Z}_{k}$ has no 2-torsion. Therefore the second Stiefel-Whitney class $w_{2}\left(M_{i}^{k}\right)$ is trivial. On the other hand, when $k$ is even the class $w_{2}\left(X_{i}\right)$ does not have the same parity of $c_{1}\left(X_{i}\right)$. Hence $M_{i}^{k}$ is not spin by Lemma 5.13 .

Notice that all these Sasaki structures are negative by construction.

Remark 5.26. The almost contact structures underlying the Sasaki manifolds $M_{i}^{k}$ defined in the proof of Theorem5.25 are inequivalent. In fact it is easy to see that their first Chern classes have different divisibilities.

Remark 5.27. The embeddings $\iota_{i}: X_{i} \longrightarrow \mathbb{C P}^{N}$ given by Kodaira Embedding Theorem depend on the complex structure on $X_{i}$. The Kähler forms $\omega_{i}$ used in the BoothbyWang construction are given by rescaling the restriction of the Fubini-Study form to $X_{i}$. Therefore, even though $\left[\omega_{1}\right]=\left[\omega_{2}\right]=x$, the Kähler forms $\omega_{1}$ and $\omega_{2}$ cannot be joined by a smooth path of symplectic forms $\omega_{t}$. In fact, in that case the complex structures on $X_{1}$ and $X_{2}$ would be deformation equivalent, at least as almost complex structures. Hence their Chern classes would agree.

\subsubsection{Simply connected higher dimensional manifolds}

In this last part of the chapter we focus on higher dimensional Sasaki manifolds with different Hodge numbers. Our next result extends Theorem 5.25 to any dimension $2 n+$ $1>3$.

Theorem 5.28. For all $n>1$ there exist countably many $(2 n+1)$-dimensional manifolds admitting two Sasaki structures with different basic Hodge numbers. Moreover, these manifolds can be arranged to be spin or non-spin and one can pick the Sasaki structures to be negative. 
Proof. The case $n=2,3$ is proved in Theorem 5.21 and Theorem 5.25.

Let $X_{1}$ and $X_{2}$ be two diffeomorphic 3-dimensional complete intersections with different first Chern class given in Table 5.1. Recall that $X_{1}$ and $X_{2}$ have different Hodge numbers by Lemma 5.24. We adopt the notation from the proof of Theorem 5.25.

Since $X_{1}$ is diffeomorphic to $X_{2}$, the projective manifolds $X_{1} \times \mathbb{C P}^{n}$ and $X_{2} \times \mathbb{C P}^{n}$ are diffeomorphic. Now consider the forms $\omega_{i}$ from Remark 5.27. Let $M_{i}^{n}$ be the BoothbyWang bundle over $\left(X_{i} \times \mathbb{C P}^{n}, \omega_{i}+\omega_{F S}\right)$.

Since the Kähler forms $\omega_{i}+\omega_{F S}$ represent the same class $x+\left[\omega_{F S}\right]$, the resulting Sasaki manifolds are diffeomorphic. Moreover, their basic Hodge numbers disagree because the Sasaki structures are regular and the Hodge numbers of $X_{1} \times \mathbb{C P}^{n}$ and $X_{2} \times$ $\mathbb{C P}^{n}$ disagree. Therefore, for all $n$ we get simply connected manifolds of dimension $2 n+1$ with different Hodge numbers.

Unfortunately the Sasaki structures constructed as Boothby-Wang bundles over $X_{i} \times$ $\mathbb{C P}^{n}$ are not of definite type. Nevertheless, we can get negative Sasaki manifolds with the same properties as follows. Consider the $k$-fold products $X_{i}^{k}=X_{i} \times \ldots \times X_{i}$. The class $(x, \ldots, x) \in H^{2}\left(X_{i}^{k}\right)=\oplus_{j=1}^{k} H^{2}\left(X_{i}\right)$ is represented by the standard product Kähler form. Hence the Boothby-Wang fibrations over $X_{1}^{k}$ and $X_{2}^{k}$ are diffeomorphic for the argument above. In this case the canonical bundle of $X_{i}^{k}$ is ample. Thus the Sasaki structures that we have constructed are negative. Moreover, their Hodge numbers disagree because the Sasaki structures are regular and the Hodge numbers of $X_{1}^{k}$ and $X_{2}^{k}$ disagree.

As in the proof of Theorem 5.25 we consider diffeomorphic complete intersections $X_{1}$ and $X_{2}$ with odd first Chern class given in Table 5.1. Now we can construct the Boothby-Wang bundle on the $k$-fold products $X_{i}^{k}=X_{i} \times \ldots \times X_{i}$ with Euler class $(l x, \ldots, l x) \in H^{2}\left(X_{i}^{k}\right)$ for $l \in \mathbb{N}$. By the same arguments above these yield two negative Sasaki structures with different basic Hodge numbers on the same differentiable manifold $M_{l}^{k}$. Moreover, Lemma 5.13 implies that $M_{l}^{k}$ is spin if and only if $l$ is odd.

Since the first Chern classes of the almost contact structures are the pullbacks under the bundle map of $c_{1}\left(X_{1}^{k}\right)$ and $c_{1}\left(X_{2}^{k}\right)$, they define elements of different order in $H^{2}\left(M_{l}^{k}\right)$. Therefore the underlying almost contact structures cannot be equivalent.

In order to obtain such examples in dimension $6 k+3$ and $6 k+5$ it is enough to reproduce the above construction on the products $X_{1}^{k} \times Y$ and $X_{2}^{k} \times Y$, where $Y$ is a complex curve, respectively surface, with ample canonical bundle.

Remark 5.29. As pointed out in Remark 5.27 the Kähler forms used in the BoothbyWang construction are given by rescaling the restriction of the Fubini-Study form to $X_{i}^{k}$. Therefore, even though they are cohomologous, they cannot be joined by a smooth path of symplectic forms $\omega_{t}$.

Remark 5.30. Theorem 5.28 cannot be extended to dimension 3. Indeed, in the threedimensional case $h_{B}^{0,0}=h_{B}^{1,1}=1$ and $h_{B}^{1,0}=\frac{1}{2} \mathrm{~b}_{B}^{1}$. Therefore, basic Hodge numbers are topological invariants of Sasakian 3-manifolds by Theorem 5.2 


\section{Chapter 6}

\section{Sasaki groups}

We consider now topological properties of K-contact and Sasakian manifolds. Specifically, we focus on fundamental groups of compact K-contact and Sasakian manifolds. In parallel with the projective and Kähler case we call these groups K-contact groups and Sasaki groups respectively. It is very interesting to understand how far the analogy between Kähler and Sasakian geometry goes in terms of fundamental groups. Following the discussion of Chapter 4 we give an interpretation of Sasaki groups as group extensions. In particular, these extensions depend on a choice of quasi-regular structure on a Sasakian manifold realizing the group. We prove that Sasaki groups enjoy many properties of projective groups. Perhaps more interestingly, we also prove that Sasaki groups do not enjoy some properties that are satisfied by Kähler and projective groups.

In Section 6.1 we present Sasaki groups as group extensions and discuss this interpretation. The remainder of the chapter is dedicated to the proof of the results presented in the introduction.

\subsection{The short exact sequence of a quasi-regular Sasaki structure}

Let $M$ be a compact Sasakian manifold and denote by $\Gamma=\pi_{1}(M)$ its fundamental group. Rukimbira's Theorem 3.57 implies that $M$ admits a quasi-regular Sasaki structure. Moreover, the Structure Theorem 3.59 shows that $M$ is a principal $S^{1}$-orbibundle over a Kähler orbifold $\mathcal{X}=(X, \mathcal{U})$. Therefore, the manifold $M$ admits a locally free $S^{1}$-action. As a consequence of Theorem 2.36 the fundamental group of a compact Sasakian manifold fits into the long exact sequence of homotopy groups

$$
\cdots \longrightarrow \pi_{2}^{o r b}(\mathcal{X}) \stackrel{\delta}{\longrightarrow} \pi_{1}\left(S^{1}\right) \cong \mathbb{Z} \longrightarrow \Gamma \longrightarrow \pi_{1}^{o r b}(\mathcal{X}) \longrightarrow 0 .
$$

In particular, every Sasaki group fits in a short exact sequence of the form

$$
0 \longrightarrow C \longrightarrow \Gamma \longrightarrow \pi_{1}^{o r b}(\mathcal{X}) \longrightarrow 0
$$


where $C=\mathbb{Z} / \operatorname{Im}(\delta)$. Therefore, the group $C$ can be as follows

$$
C \cong \begin{cases}0, & \text { if } \delta \text { is surjective } \\ \mathbb{Z}, & \text { if } \delta \text { is trivial. } \\ \mathbb{Z}_{k}, & \text { otherwise. }\end{cases}
$$

Hence we have an associated fibration of classifying spaces

$$
\pi_{\Gamma}: B \Gamma \longrightarrow B \pi_{1}^{o r b}(\mathcal{X})
$$

with fiber $B C$.

Suppose now that the map $\delta: \pi_{2}^{\text {orb }}(\mathcal{X}) \longrightarrow \mathbb{Z}$ is trivial, i.e. assume $C=\mathbb{Z}$. Then we get a principal $S^{1}$-bundle $\pi_{\Gamma}: B \Gamma \longrightarrow B \pi_{1}^{o r b}(\mathcal{X})$ which is classified by its Euler class $e(\Gamma)$. By definition principal $S^{1}$-orbibundles $P$ on $X$ correspond to principal $S^{1}$-bundles $\widetilde{P}$ on $B X$. In particular, the Euler classes of $P$ and $\widetilde{P}$ coincide. Consider the principal bundle $\tilde{M}$ on $B \mathcal{X}$ determined by $\pi: M \longrightarrow \mathcal{X}$. Notice that $\pi_{1}(\tilde{M})=\Gamma$ so that we can obtain $B \Gamma$ from $\widetilde{M}$ by glueing cells of dimension $m>2$ as explained in Section 4.1 . It is easy to see that the bundle $\tilde{M}$ is the restriction of $\left(\mathrm{B} \Gamma, \pi_{\Gamma}\right)$ to $B \mathcal{X} \subset B \pi_{1}^{o r b}(\mathcal{X})$. Equivalently, the bundle $\tilde{M}$ is the pullback of $(6.3)$ under $\iota$; see $(4.3)$ for the definition of $\iota$. This follows directly from the construction of the classifying space in the orbifold case. Visually we have

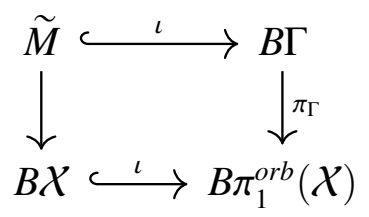

where the vertical arrows are principal $S^{1}$-bundles and the horizontal maps are defined in (4.3).

Thus we identify the principal $S^{1}$-orbibundle $\pi: M \longrightarrow \mathcal{X}$ with the pullback of $\left(\mathrm{B} \Gamma, \pi_{\Gamma}\right)$ under $\iota: B \mathcal{X} \longrightarrow B \pi_{1}^{o r b}(\mathcal{X})$. In particular, by naturality of the Euler class, we have proven the following

Proposition 6.1. Let $M$ be a compact Sasakian manifold and $\Gamma=\pi_{1}(M)$. Assume that $M$ is endowed with a quasi-regular Sasaki structure $\pi: M \longrightarrow \mathcal{X}$ such that the associated central extension defined above has the form

$$
0 \longrightarrow \mathbb{Z} \longrightarrow \Gamma \longrightarrow \pi_{1}^{\text {orb }}(\mathcal{X}) \longrightarrow 0
$$

Then we have $[\omega]=\iota^{*}(e(\Gamma)) \in H_{\text {orb }}^{2}(\mathcal{X} ; \mathbb{Z})$.

We discuss now the map $\delta: \pi_{2}^{o r b}(\mathcal{X}) \longrightarrow \mathbb{Z}$ to describe the group $C$ in geometrical terms. In order to give an explicit description of the homomorphism $\delta$, we consider the universal bundle $S^{\infty} \longrightarrow \mathbb{C P}^{\infty}$. The principal $S^{1}$-bundle $\widetilde{M} \longrightarrow B \mathcal{X}$ is the pullback of 
$S^{\infty}$ under the classifying map $f: B \mathcal{X} \longrightarrow \mathbb{C P}^{\infty}$. By naturality, we get a map between the long exact sequences of homotopy groups yielding the commutative diagram

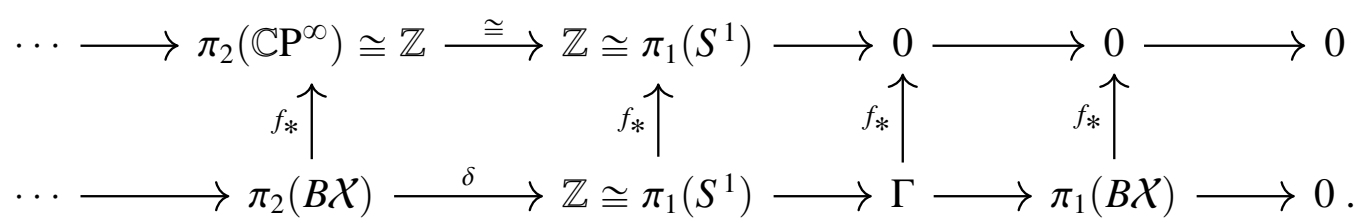

We focus on the isomorphism $\pi_{2}\left(\mathbb{C P}^{\infty}\right) \longrightarrow \pi_{1}\left(S^{1}\right)$. This clearly factorizes through the Hurewicz map $\pi_{2}\left(\mathbb{C P}^{\infty}\right) \stackrel{h}{\longrightarrow} H_{2}\left(\mathbb{C P}^{\infty} ; \mathbb{Z}\right)$ to give

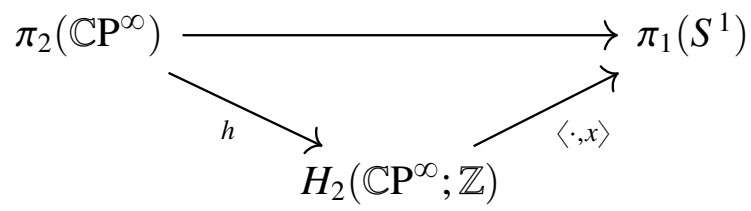

where the map $\langle\cdot, x\rangle$ is capping with the generator $x \in H^{*}\left(\mathbb{C P}^{\infty} ; \mathbb{Z}\right)$. By definition the Euler class $e(\tilde{M})=[\omega]$ of the bundle $\widetilde{M} \longrightarrow B X$ is the pullback $f^{*}(x)$. From the diagram

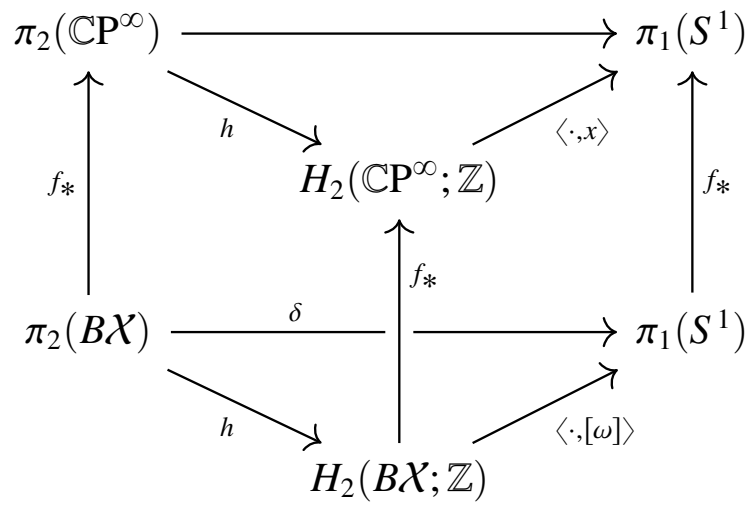

we conclude that $\delta(S)=\left\langle f_{*}(S), x\right\rangle=\langle S,[\omega]\rangle$ for any element $S \in \pi_{2}(B \mathcal{X})$. We summarize the above discussion in the following:

Lemma 6.2. Let $M$ be a compact Sasakian manifold. Denote by $\pi: M \longrightarrow X$ the principal $S^{1}$-bundle over a Kähler orbifold $(\mathcal{X}, \omega)$ determined by a quasi-regular Sasaki structure on $M$. Then the map $\delta: \pi_{2}^{\text {orb }}(\mathcal{X}) \longrightarrow \mathbb{Z}$ in the long exact sequence of homotopy groups

$$
\cdots \longrightarrow \pi_{2}^{o r b}(\mathcal{X}) \stackrel{\delta}{\longrightarrow} \pi_{1}\left(S^{1}\right) \cong \mathbb{Z} \longrightarrow \Gamma \longrightarrow \pi_{1}^{o r b}(\mathcal{X}) \longrightarrow 0
$$


is given by the composition

$$
\pi_{2}^{o r b}(\mathcal{X}) \stackrel{h}{\longrightarrow} H_{2}^{\text {orb }}(\mathcal{X} ; \mathbb{Z}) \stackrel{\langle\cdot,[\omega]\rangle}{\longrightarrow} \pi_{1}\left(S^{1}\right) \cong \mathbb{Z}
$$

where $h$ is the Hurewicz map and the map $\langle\cdot,[\omega]\rangle$ is evaluation on the Euler class of the bundle $\pi: M \longrightarrow X$.

Let us now come back to the extension (6.1). In particular, we want to relate the orbifold fundamental group $\pi_{1}^{o r b}(\mathcal{X})$ to a genuine projective group. Note that the map $p: B X \longrightarrow X$ defined in Section 2.3 induces a surjective map $p_{*}$ at the level of fundamental groups. Moreover, the kernel of $p_{*}$ is normally generated by loops around the irreducible divisors $D_{i}$ contained in the singular set of $\mathcal{X}$, see Section 2.4. These loops represent torsion elements of order $m_{i}$ the ramification index of $D_{i}$. Therefore the kernel $K$ of the map $p_{*}: \pi_{1}^{o r b}(\mathcal{X}) \longrightarrow \pi_{1}(X)$ is generated by (possibly infinitely many) torsion elements.

Assume now that $K$ is finitely generated. Then by Remark 4.5 we get an isomorphism

$$
H^{*}\left(\pi_{1}^{o r b}(\mathcal{X}) ; \mathbb{R}\right) \cong H^{*}\left(\pi_{1}(X) ; \mathbb{R}\right) .
$$

Moreover, $X$ admits a resolution which preserves the fundamental group by a result of Kollár, see [72, Theorem 7.5.2]. Thus the real cohomology ring of $\pi_{1}^{o r b}(\mathcal{X})$ is that of a projective group. Notice that whenever $C \neq \mathbb{Z}$ we have an isomorphism

$$
H^{*}\left(\pi_{1}^{o r b}(\mathcal{X}) ; \mathbb{R}\right) \cong H^{*}(\Gamma ; \mathbb{R}) .
$$

In this instance $\Gamma$ itself has the cohomology ring of the projective group $\pi_{1}(X)$. We have proven the following:

Lemma 6.3. For any quasi-regular structure $\pi: M \longrightarrow \mathcal{X}$ on a Sasakian manifold $M$ one has the diagram

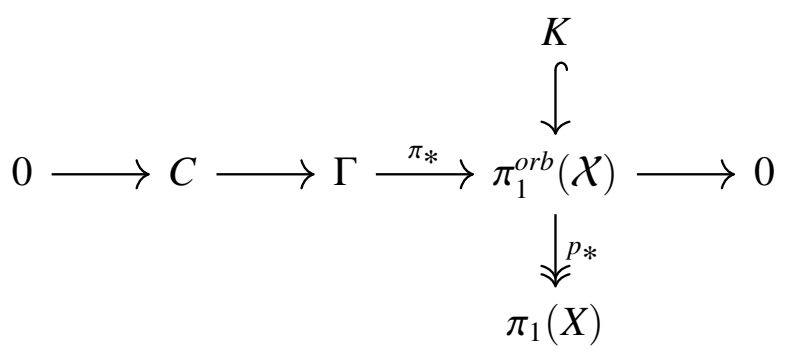

where $\Gamma=\pi_{1}(M)$. Moreover, $\pi_{1}(X)$ is a projective group and the kernel $K$ of $p_{*}$ is generated by torsion elements. If $K$ is finitely generated by torsion elements, then $H^{*}\left(\pi_{1}^{\text {orb }}(\mathcal{X}) ; \mathbb{R}\right) \cong H^{*}\left(\pi_{1}(X) ; \mathbb{R}\right)$. If in addition $C \neq \mathbb{Z}$, then $H^{*}\left(\pi_{1}(X) ; \mathbb{R}\right) \cong H^{*}(\Gamma ; \mathbb{R})$.

Remark 6.4. The results of this section are stated and proved in the Sasakian setting. Nevertheless, it is easy to check that none of the arguments in the proofs of Propo- 
sition 6.1 and Lemma 6.2 uses integrability of the CR structure. Therefore, Proposition 6.1 and Lemma 6.2 hold true in the K-contact setting.

\subsection{Realizability}

This section is dedicated to realizability results. Namely, we discuss which groups are Sasaki groups and in which dimension they can be realized. In particular, we show that $\mathcal{S}_{2 n+1}$ is not contained in $\bigcup_{m>n} \mathcal{S}_{2 m+1}$ for small $n$.

We begin this section by investigating in which dimensions projective groups, respectively finitely presentable groups, can be realized as fundamental groups of compact Sasakian manifolds, resp. K-contact manifolds.

Theorem 6.5. Every projective group $\Gamma$ can be realized as the fundamental group of a compact Sasakian manifold of any odd dimension $2 n+1 \geqslant 5$.

Proof. Let $\Gamma$ be a projective group. By the discussion in Section 4.2, we can assume $\Gamma=\pi_{1}(X)$ where $X$ is a smooth projective variety of any (real) dimension $2 n \geqslant 4$. Denote by $[\omega]$ the integral Kähler class of $X$. The blow-up of $X$ at a point, topologically $X \# \overline{\mathbb{C P}^{n}}$, can be endowed with the integral Kähler class $k[\omega]+E$ where $E$ is the Poincaré dual of the exceptional divisor $D$ and $k \in \mathbb{N}$ is large enough.

Consider now the Boothby-Wang fibration $M$ over $X \# \overline{\mathbb{C P}^{n}}$ with Euler class $e=$ $k[\omega]+E$ and the associated long exact sequence

$$
\cdots \longrightarrow \pi_{2}\left(X \# \overline{\mathbb{C P}^{n}}\right) \stackrel{\delta}{\longrightarrow} \pi_{1}\left(S^{1}\right) \longrightarrow \pi_{1}(M) \longrightarrow \Gamma \longrightarrow 0
$$

The exceptional divisor $D \cong \mathbb{C P}^{n-1}$ contributes a non-torsion spherical class $[D]$ to $H_{2}(X \# \overline{\mathbb{C P}} ; \mathbb{Z})$ on which the Euler class evaluates to 1 . By Lemma 6.2 the map $\delta$ is surjective, that is, we get the desired isomorphism $\pi_{1}(M) \cong \Gamma$.

Remark 6.6. Chen [30] proved that all projective groups are Sasaki, cf. Theorem 4.26 However, his proof only realizes these groups as fundamental groups of Sasakian manifolds of dimension $2 n+1 \geqslant 7$ because it relies on the join construction.

Theorem 6.7. Every finitely presentable group $\Delta$ can be realized as the fundamental group of a compact $K$-contact manifold of any odd dimension $2 n+1 \geqslant 5$.

Proof. The proof is similar to that of Theorem 6.5. Namely, we replace the projective variety $X$ by a symplectic manifold.

Let $\Delta$ be any finitely presented group. By a celebrated theorem of Gompf [50] there exists a closed symplectic $2 n$-manifold $Y$ such that $\pi_{1}(Y)=\Delta$, for any $n \geqslant 2$. Since non-degeneracy is an open condition, there exists a symplectic form on $Y$ representing a rational class in cohomology. After multiplication with a large integer we may assume 
that $Y$ is equipped with a symplectic form $\omega$ representing an integral class $[\omega]$, see Remark 3.23 .

After possibly replacing $Y$ by its symplectic blow-up at a point, there exists a spherical class in $H_{2}(Y ; \mathbb{Z})$ on which $[\omega]$ evaluates to 1 . Therefore, the Boothby-Wang fibration over $Y$ with Euler class $[\omega]$ is a compact $2 n+1$-dimensional K-contact manifold $N$ with $\pi_{1}(N)=\Delta$.

Remark 6.8. It was shown in [10] that all finitely presentable groups are K-contact, cf. Theorem 4.27. However, the proof only realizes these groups as fundamental groups of K-contact manifolds of dimension $2 n+1 \geqslant 7$ because it relies on the join construction.

Remark 6.9. Theorem 3.94 implies that K-contact groups in dimension 3 are Sasaki groups. Moreover, three-dimensional Sasaki manifolds were classified by Geiges [48]. As a result their fundamental groups are lattices in $S O(4), \widetilde{S} L(2, \mathbb{R})$ or the real Heisenberg group $\mathcal{H}_{3}$. This shows that the bounds on the dimension in Theorem 6.5 and Theorem 6.7 are optimal.

As a motivation to discuss the quasi-regular setting, let us rephrase the above results. Consider the central extension (6.1) associated to a regular Sasaki structure:

$$
0 \longrightarrow C \longrightarrow \Gamma \longrightarrow \pi_{1}(X) \longrightarrow 0 \text {. }
$$

Here $\pi_{1}(X)$ is the ordinary fundamental group of a compact projective manifold. The Boothby-Wang Theorem ensures that all projective groups figure as the last term of the extension (6.1) associated to some regular Sasaki structure. Moreover, Theorem 6.5 shows that every projective group is the fundamental group of a compact regular Sasaki manifold. We can then rephrase Theorem 6.5 as: every group which appears at the right of a central extension associated to a regular Sasaki structure also figures in the middle of some other such extension.

It is then natural to ask whether the analogous statement holds true in the quasiregular case. Our next theorem gives an affirmative answer to this question.

Theorem 6.10. Let $M$ be a $2 n+1$-dimensional compact quasi-regular Sasaki manifold and let

$$
0 \longrightarrow C \longrightarrow \Gamma \longrightarrow \pi_{1}^{o r b}(\mathcal{X}) \longrightarrow 0
$$

be the associated central extension. Then $\pi_{1}^{\text {orb }}(\mathcal{X})$ is a Sasaki group.

Proof. The proof is a generalization to the orbifold setting of the argument in Theorem 6.5 .

Let $\pi: M \rightarrow \mathcal{X}$ be the $S^{1}$-orbibundle with Euler class $[\omega]$ associated to the quasiregular structure on $M$. Consider the blow-up of $\mathcal{X}$ at a smooth point. This gives a projective orbifold $\mathcal{Y}$ with underlying topological space $Y=X \# \overline{\mathbb{C P}} \bar{P}^{n}$. Clearly $\pi_{1}^{\text {orb }}(\mathcal{X})=$ $\pi_{1}^{o r b}(\mathcal{Y})$. Moreover, we can endow $\mathcal{Y}$ with an integral Kähler class $e=k[\omega]+E$ where $E$ is the Poincaré dual to the exceptional divisor $D \cong \mathbb{C P}^{n-1}$ and $k$ is a positive, large enough integer. 
We make use of Theorem 2.53 to ensure that we can pick $k$ such that the principal $S^{1}$-bundle associated to $e \in H_{\text {orb }}^{2}(\mathcal{Y} ; \mathbb{Z})$ is smooth. For instance, it is enough to take $k$ relatively prime to the orders of the uniformizing groups of $\mathcal{X}$. Let $N$ denote this bundle. By the Structure Theorem 3.59, $N$ is a Sasaki manifold. We want to prove that $\pi_{1}(N)=\pi_{1}^{o r b}(\mathcal{Y})$.

Now consider the map $p_{*}: \pi_{2}^{\text {orb }}(\mathcal{Y}) \longrightarrow \pi_{2}^{\text {orb }}(\mathcal{X})$ induced by the blow-down map $p: \mathcal{Y} \longrightarrow \mathcal{X}$. Let $f: S^{2} \longrightarrow B \mathcal{Y}$ be a representative of the generator of $\operatorname{ker}\left(p_{*}\right)$. By construction the pullback of $N$ under $f$ is the Hopf bundle $S^{3} \longrightarrow S^{2}$. Therefore, $f$ induces the following map of long exact sequences

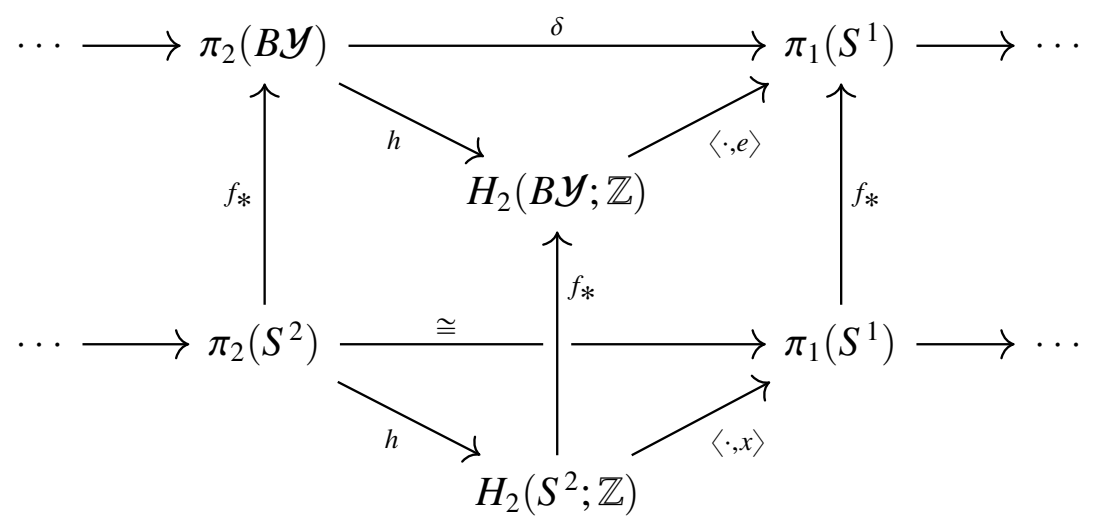

where $x$ is the generator of $H^{2}\left(S^{2} ; \mathbb{Z}\right)$ given by the orientation. Since $x=f^{*}(e)$, it follows that the map $\delta: \pi_{2}^{o r b}(\mathcal{Y}) \longrightarrow \pi_{1}\left(S^{1}\right)$ is surjective. We conclude that $\pi_{1}(N)=$ $\pi_{1}^{o r b}(\mathcal{Y})=\pi_{1}^{o r b}(\mathcal{X})$.

Remark 6.11. The orbifold fundamental group of a compact orbifold is finitely presentable. Since every such group is the fundamental group of a symplectic manifold, the K-contact analogue of Theorem 6.10 is, in fact, weaker than Theorem 6.7.

A natural question to ask on Sasaki groups is the following:

Question: In which dimension does there exist a Sasakian manifold $M$ with $\pi_{1}(M)=\Gamma$ for a given Sasaki group $\Gamma$ ?

Theorem 6.5 together with Remark 6.9 provide an answer to this question when $\Gamma$ is also a projective group. In the Kähler case the analogous problem has a partial answer in Proposition 4.9. However, in the Sasakian setting taking products with simply connected manifolds does not yield Sasaki manifolds in a natural way. Moreover, consider a quasi-regular Sasaki structure such that the map $\delta$ of Lemma 6.2 is surjective. Then the Euler class $e$ of the associated group extension pulls back to the Kähler class of $\mathcal{X}$ by Proposition 6.1. Hence the powers $e^{k}$ of the Euler class cannot vanish for $k \leqslant \operatorname{dim}_{\mathbb{C}}(\mathcal{X})$. This suggests that some dimensional restrictions may apply. Our next result exploits this observation to prove that the Sasakian analogue of ii) in Proposition 4.9 is false. 
Theorem 6.12. The set $\mathcal{S}_{2 n+1}$ is not contained in $\bigcup_{m>n} \mathcal{S}_{2 m+1}$ for $n=1,2,3$.

Proof. Fix $n \in\{1,2,3\}$. We want to find elements of $\mathcal{S}_{2 n+1}$, i.e., fundamental groups of a compact Sasakian manifold of dimension $2 n+1$, which cannot be realized as $\pi_{1}(M)$ for $M$ a Sasakian manifold of dimension larger than $2 n+1$. In particular we will show that this property is satisfied by the integral Heisenberg groups of dimension 3,5 and 7.

We have seen in Example 4.20 that the Heisenberg group $\mathcal{H}_{2 n+1}$ arises as the fundamental group of the Sasaki manifold given by the Boothby-Wang fibration over the $2 n$-dimensional torus $T^{2 n}$. Thus $\mathcal{H}_{2 n+1} \in \mathcal{S}_{2 n+1}$.

Now suppose $\mathcal{H}_{2 n+1}=\pi_{1}(M)$ where $M$ is a Sasakian manifold of dimension $2 m+1$. A quasi-regular structure on $M$ yields a central extension of the form:

$$
0 \longrightarrow C \stackrel{\varphi}{\longrightarrow} \mathcal{H}_{2 n+1} \longrightarrow \pi_{1}^{o r b}(\mathcal{X}) \longrightarrow 0
$$

First we prove by contradiction that $C \neq 0$ and $C \neq \mathbb{Z}_{k}$ leaving as the only possibility $C=\mathbb{Z}$, see 6.2 . Since $\mathcal{H}_{2 n+1}$ is torsion-free, $C$ cannot be a non-trivial finite group. Now suppose that $C$ is the trivial group. By Lemma $6.3 \pi_{1}^{\text {orb }}(X)$ surjects onto $\pi_{1}(X)$ with kernel generated by elements of finite order. Hence $\mathcal{H}_{2 n+1} \cong \pi_{1}^{\text {orb }}(\mathcal{X})$ and torsion-freeness yields an isomorphism $\mathcal{H}_{2 n+1} \cong \pi_{1}(X)$. Thus $\mathcal{H}_{2 n+1}$ is a Kähler group, contradicting the results of [27].

The only possibility left is $C \cong \mathbb{Z}$. We will first treat the case where $\varphi(1)=z$ is a generator of the center of $\mathcal{H}_{2 n+1}$. In this case the central extension (6.6) reads

$$
0 \longrightarrow \mathbb{Z} \longrightarrow \mathcal{H}_{2 n+1} \longrightarrow \mathbb{Z}^{2 n} \longrightarrow 0
$$

with Euler class $e \in H^{2}\left(\mathbb{Z}^{2 n} ; \mathbb{Z}\right)$. Let $B \mathbb{Z}^{2 n}$ be the classifying space obtained by attaching cells to $B X$ as explained in Section 4.1. By Proposition 6.1 the Euler class $e$ of the extension above is mapped to the Euler class $[\omega]$ of the $S^{1}$-orbibundle $\pi: M \longrightarrow \mathcal{X}$ by the injection $\iota^{*}: H^{2}\left(B \mathbb{Z}^{2 n} ; \mathbb{Z}\right) \hookrightarrow H_{\text {orb }}^{2}(\mathcal{X} ; \mathbb{Z})$. Thus

$$
0=\iota^{*}\left(e^{n+1}\right)=\iota^{*}(e)^{n+1}=[\omega]^{n+1} .
$$

On the other hand, $\omega$ is a Kähler form on a $2 m$-dimensional orbifold so that $[\omega]^{l} \neq 0$ for all $l \leqslant m$. We conclude that $m \leqslant n$.

If we are not in the preceding case, then $\varphi(1)=z^{k}$ so that the sequence (6.6) reads

$$
0 \longrightarrow \mathbb{Z} \longrightarrow \mathcal{H}_{2 n+1} \longrightarrow G_{k} \longrightarrow 0
$$

where, in turn, $G_{k}$ fits in the short exact sequence

$$
0 \longrightarrow \mathbb{Z}_{k} \longrightarrow G_{k} \longrightarrow \mathbb{Z}^{2 n} \longrightarrow 0 \text {. }
$$

Again by Proposition 6.1, $\iota^{*}\left(e_{k}\right)=[\omega]$ where $e_{k}$ is the Euler class of the central extension 6.7). The class $e_{k}$ is not torsion because $[\omega]$ is a Kähler class and $\iota^{*}$ is injective. Moreover, the Lyndon-Hochschild-Serre spectral sequence for $\mathbb{Z}_{k} \subset G_{k}$ gives 
$H^{*}\left(\mathbb{Z}^{2 n} ; \mathbb{R}\right) \cong H^{*}\left(G_{k} ; \mathbb{R}\right)$, hence $e_{k}^{l}=0$ for all $l>n$. As in the previous case, we get $0=\iota^{*}\left(e_{k}^{n+1}\right)=\iota^{*}\left(e_{k}\right)^{n+1}=[\omega]^{n+1}$.

This proves that $\mathcal{H}_{2 n+1} \notin \mathcal{S}_{2 m+1}$ for $m>n$ and concludes the proof.

Remark 6.13. In order to show that $\mathcal{S}_{3} \nsubseteq \bigcup_{m>1} \mathcal{S}_{2 m+1}$, it is enough to consider a $\mathbb{Z}$ central extension $\Delta_{g, e}$ of the fundamental group $\Gamma_{g}$ of a closed orientable surface $\Sigma_{g}$ of genus $g \geqslant 1$ with non-trivial Euler class $e \in H^{2}\left(\Gamma_{g} ; \mathbb{Z}\right)=H^{2}\left(\Sigma_{g} ; \mathbb{Z}\right)$. This is the fundamental group of the principal $S^{1}$-bundle over $\Sigma_{g}$ with Euler class $e$, thus a Sasaki group. On the other hand, $\Delta_{g, e}$ is not Kähler since the cup product

$$
H^{1}\left(\Delta_{g, c} ; \mathbb{R}\right) \times H^{1}\left(\Delta_{g, c} ; \mathbb{R}\right) \longrightarrow H^{2}\left(\Delta_{g, c} ; \mathbb{R}\right)
$$

is degenerate, cf. Proposition 4.17.

In fact, we will see in Corollary 6.23 that this is the case for any infinite Sasaki group $\Gamma \in \mathcal{S}_{3}$.

Remark 6.14. One cannot use higher dimensional Heisenberg groups $\mathcal{H}_{2 n+1}$ to prove the same result for $\mathcal{S}_{2 n+1}$ with $n \geqslant 4$. In fact Campana [20] proved that these groups are projective. Therefore, they are Sasaki groups realizable in any odd dimension $2 n+1 \geqslant 5$ by Theorem 6.5 .

\subsection{Direct products}

Recall that the class of Kähler groups is closed under direct products because the product of Kähler manifold is again a Kähler manifold. Motivated by this rather elementary property of Kähler groups in this section we study direct products of Sasaki groups. Products of Sasakian manifolds are not Sasakian for dimension reasons. Nevertheless, one can ask whether or not the set of Sasaki groups is closed under direct products. A natural approach is to try to perform a construction, e.g. a join construction, while controlling the fundamental group. It turns out that this method cannot work because the set of Sasaki groups is not closed under direct products.

Theorem 6.15. The set $\mathcal{S}$ is not closed under direct products.

Proof. Let $\Sigma_{g}$ be the closed orientable surface of genus $g \geqslant 2$ and denote its fundamental group by $\Gamma_{g}$. We will show that, even though $\mathcal{H}_{3}$ and $\Gamma_{g}$ are Sasaki groups, their product $\mathcal{H}_{3} \times \Gamma_{g}$ cannot be the fundamental group of any compact Sasakian manifold.

Assume there were a compact Sasakian manifold $M$ with $\pi_{1}(M)=\mathcal{H}_{3} \times \Gamma_{g}$. Note that $\pi_{1}(M)$ is not a Kähler group by Proposition 4.17. Thus, by the same argument in the proof of Theorem 6.12, we would have the following central extension:

$$
0 \longrightarrow \mathbb{Z} \longrightarrow \pi_{1}(M) \longrightarrow G_{k} \times \Gamma_{g} \longrightarrow 0
$$

where $G_{k}$ is a $\mathbb{Z}_{k}$-extension of $\mathbb{Z}^{2}$ (with possibly $k=1$ and $G_{k}=\mathbb{Z}^{2}$ ). Again the Lyndon-Hochschild-Serre spectral sequence for $\mathbb{Z}_{k} \subset G_{k} \times \Gamma_{g}$ yields $H^{*}\left(\mathbb{Z}^{2} \times \Gamma_{g} ; \mathbb{R}\right) \cong$ 
$H^{*}\left(G_{k} \times \Gamma_{g} ; \mathbb{R}\right)$. Furthermore, the extension 6.8 is the pullback of the extension 6.7 ) for $n=1$ via the projection on the first factor. That is, we have the following pullback diagram:

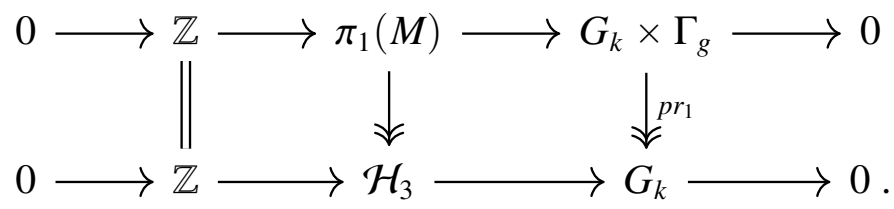

Therefore the characteristic class $p r_{1}^{*}\left(e_{k}\right)=d_{k} \in H^{2}\left(B G_{k} \times \Gamma_{g} ; \mathbb{Z}\right)$ of the extension 6.8$)$ satisfies $d_{k}^{2}=0$. This implies $\operatorname{dim}(\mathcal{X})=2$, that is, the underlying space $X$ is a closed surface. Thus $\mathrm{b}_{2}(\mathcal{X})=\mathrm{b}_{2}(X)=1$ because $H_{\text {orb }}^{*}(X ; \mathbb{R})=H^{*}(X ; \mathbb{R})$, see Remark 2.27 . Moreover, the injection (4.5) in the orbifold case implies $\mathrm{b}_{2}\left(G_{k} \times \Gamma_{g}\right) \leqslant \mathrm{b}_{2}(X)$. However, $\mathrm{b}_{2}\left(G_{k} \times \Gamma_{g}\right)=\mathrm{b}_{2}\left(\mathbb{Z}^{2} \times \Gamma_{g}\right)>1$. Therefore $G_{k} \times \Gamma_{g}$ is not the orbifold fundamental group of an orbifold surface. Hence $\mathcal{H}_{3} \times \Gamma_{g}$ is not a Sasaki group.

Remark 6.16. Let $\Delta_{g, e}$ be the groups defined in Remark 6.13. The same argument proves that none of the products $\Delta_{g, e} \times \Gamma_{g}$ is a Sasaki group. More generally, the product $\Delta \times \Gamma_{g}$ is not Sasaki if $\Delta$ is an infinite Sasaki group in dimension 3, see 6.21.

Remark 6.17. An alternative proof of Theorem 6.15 follows by combining [69, Proposition 7.2] with the classification of 3-dimensional Sasakian manifolds in [48]. In order to see this, let $H$ be the Boothby-Wang bundle over $T^{2}$ with Euler class a generator of $H^{2}\left(T^{2}\right)$. Then Proposition 7.2 in [69] implies that for any compact even dimensional manifold $M$ the product $M \times H$ is not Sasakian. However, the proof of this proposition shows more. Namely, it proves that $\pi_{1}(M) \times \mathcal{H}_{3}$ is not realizable as the fundamental group of a Sasakian manifold of dimension $2 n+1 \geqslant 5$. On the other hand, the classification of 3-dimensional Sasakian manifolds in [48] ensures that $\mathcal{H}_{3} \times \Gamma_{g}$ is not the fundamental group of a Sasakian manifold of dimension 3.

\subsection{Free products}

We focus now on instances in which Sasaki and Kähler groups present similar behaviour. We are mainly interested in the relation with free products. Specifically, in this section we prove that free products of groups with a non-trivial finite quotient cannot be Sasaki. In fact, we show more, i.e., we give the Sasakian analogue of Theorem 4.22 . Namely, we prove that under mild hypotheses on $\Gamma_{1}$ and $\Gamma_{2}$ the group $\left(\Gamma_{1} * \Gamma_{2}\right) \times H$ is not Sasaki for any group $H$. This shows in particular that Sasaki groups are indecomposable under free products whose factors have non-trivial finite quotients. The proof we give here is an adaptation to the Sasakian setting of an argument from the Kähler case; see the proof of Theorem 4.22 .

Theorem 6.18. Let $\Gamma_{1}$ and $\Gamma_{2}$ be two groups. Assume $f_{i}: \Gamma_{i} \longrightarrow Q_{i}$ is a non-trivial quotient with $\left|Q_{i}\right|=m_{i}<\infty$ for $i=1,2$. 
a) Then $\left(\Gamma_{1} * \Gamma_{2}\right)$ is not Sasaki.

b) For any group $H$ the product $\left(\Gamma_{1} * \Gamma_{2}\right) \times H$ is not Sasaki.

Proof. Clearly part $a$ ) follows from the second claim so we prove only $b$ ). Set $\Gamma=$ $\left(\Gamma_{1} * \Gamma_{2}\right) \times H$. The proof is divided into two cases.

Case $1\left(b_{1}(H)\right.$ even $)$ By Lemma 4.21, there exists a finite index subgroup $\Delta \subset \Gamma_{1} * \Gamma_{2}$ with $\mathrm{b}_{1}(\Delta)$ odd. Hence, the group $\Delta \times H$ is a finite index subgroup of $\Gamma$ with odd first Betti number. Thus $\Gamma$ cannot be Sasaki.

Case $2\left(b_{1}(H)\right.$ odd $)$ In this case $b_{1}\left(\Gamma_{1} * \Gamma_{2}\right)>0$. Then we can assume that the first Betti number of $\Gamma_{1}$ is positive. The proof of Lemma 4.21 provides a finite index subgroup of $\Gamma_{1} \times \Gamma_{2}$ of the form $F_{n} * G$. Moreover, the rank of $F_{n}$ can be chosen to be arbitrarily large. Since the class of Sasaki groups is closed under taking finite index subgroups, we can assume $\Gamma=\left(F_{n} * G\right) \times H$ with $n>\mathrm{b}_{1}(H)$.

Let $M$ be a compact Sasakian manifold with $\pi_{1}(M)=\Gamma$. Consider a quasi-regular Sasaki structure $\pi: M \longrightarrow \mathcal{X}$ and let

$$
0 \longrightarrow C \longrightarrow \Gamma \longrightarrow \pi_{1}^{o r b}(\mathcal{X}) \longrightarrow 0
$$

be the associated central extension. Since $C$ is mapped to the center of $\Gamma$, the extension is a pullback of the following form

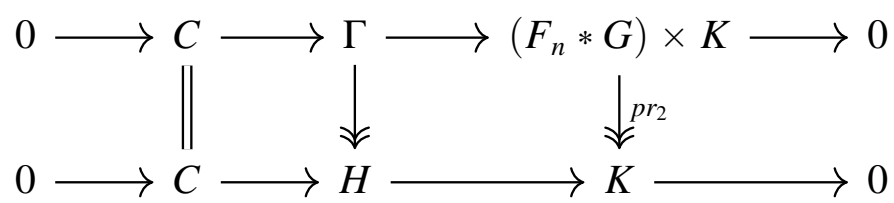

where $p r_{2}$ is the projection on the second factor and $\pi_{1}^{o r b}(\mathcal{X})=\left(F_{n} * G\right) \times K$. Now $H^{1}\left(\pi_{1}^{o r b}(\mathcal{X}) ; \mathbb{R}\right)$ is endowed with a skew-symmetric non-degenerate bilinear product by Proposition 4.18. Moreover, this factorizes through the cup product, i.e.

$$
H^{1}\left(\pi_{1}^{o r b}(\mathcal{X}) ; \mathbb{R}\right) \times H^{1}\left(\pi_{1}^{o r b}(\mathcal{X}) ; \mathbb{R}\right) \stackrel{\cup}{\longrightarrow} H^{2}\left(\pi_{1}^{o r b}(\mathcal{X}) ; \mathbb{R}\right) \longrightarrow \mathbb{R}
$$

Therefore, $H^{1}\left(F_{n}\right)$ is an isotropic subspace in $H^{1}\left(\pi_{1}^{\text {orb }}(\mathcal{X})\right) \cong H^{1}\left(F_{n}\right) \oplus H^{1}(G) \oplus$ $H^{1}(K)$ which is orthogonal to $H^{1}(G)$. We also have that $\mathrm{b}_{1}(K)=\mathrm{b}_{1}(H)$ since $\mathrm{H}$ is a non-trivial cyclic central extension of $K$. Now the inequality $n>\mathrm{b}_{1}(H)=\mathrm{b}_{1}(K)$ implies that the skew-symmetric product is degenerate. We conclude that $\Gamma$ is not a Sasaki group.

Remark 6.19. Sasaki manifolds do satisfy the Hard Lefschetz Theorem 3.97 but this does not induce a non-degenrate bilinear product as in Proposition 4.17. In particular, such a bilinear map would not factorize through the cup product as Example 4.19 shows. 
Remark 6.20. Theorem 4.30 implies that Sasaki groups are indecomposable under free products. In particular, it implies part a) of Theorem 6.18. However, it does not imply part b) of Theorem 6.18. Moreover, the proof of Theorem 6.18 does not rely on [21, 22], cf. Remark 4.31

\subsection{Three-manifold groups}

Motivated by the results on free groups and free products we discuss the relation between Sasaki and 3-manifold groups. These groups enjoy different properties than Kähler groups. For instance, by taking connected sums one sees that 3-manifolds groups are closed under free products. It was proved in [79] that an infinite 3-manifold group is also a Kähler group if and only if it is a surface group, see Theorem 4.23 . Clearly the fundamental group of a compact 3-dimensional Sasakian manifold lies in the intersection of 3-manifold groups and Sasaki groups. Thus the analogous statement to Theorem 4.23 in the Sasakian setting involves only Sasaki groups realizable in dimension 5 or higher, that is, it only involves the sets $\mathcal{S}_{2 n+1}$ for $n \geqslant 2$. It turns out that Sasaki groups have very little in common with 3-manifold groups, apart from the obvious intersection given by surface groups and some finite groups.

Theorem 6.21. Let $\Gamma$ be an infinite 3-manifold group. Then $\Gamma \in \mathcal{S}_{2 n+1}$ for some $n \geqslant 2$ if and only if $\Gamma$ is the fundamental group of a closed orientable surface.

Proof. Throughout this proof $N$ will denote a (not necessarily closed) 3-manifold.

All fundamental groups of closed orientable surfaces are projective, thus the necessity follows from Theorem 6.5 .

Now let $\Gamma=\pi_{1}(N)$ where $N$ is a 3-manifold and $|\Gamma|=\infty$.

Suppose $\Gamma$ is the fundamental group of a closed Sasakian manifold $M$ of dimension larger than 3 . In particular, $\Gamma$ is finitely presentable and, by a result of Jaco [64], we can assume that $N$ is compact.

Now suppose $N$ is not prime. This means that $\Gamma=\Gamma_{1} * \Gamma_{2}$ for two three-manifold groups $\Gamma_{1}$ and $\Gamma_{2}$. The groups $\Gamma_{1}$ and $\Gamma_{2}$ satisfy the hypothesis of Lemma 4.21 because 3 -manifold groups are residually finite, see [4]. Thus $\Gamma$ has a finite index subgroup with odd first Betti number contradicting the assumption that $\Gamma$ is Sasaki. Hence we can assume that $N$ is prime.

Moreover, if $\Gamma$ is virtually cyclic, then it is not a Sasaki group by parity of the first Betti number. Hence we can assume $N$ to be irreducible as a consequence of the sphere theorem [86]. This in particular implies that $N$ is aspherical and $\Gamma$ is torsion-free.

Now consider the central extension (4.2) associated to a quasi-regular structure on $M$ :

$$
0 \longrightarrow C \longrightarrow \Gamma \longrightarrow \pi_{1}^{o r b}(\mathcal{X}) \longrightarrow 0
$$

Since $\Gamma$ is torsion-free, $C$ cannot be a non-trivial finite cyclic group. Moreover, if $C$ is the trivial group, then $\Gamma$ is isomorphic $\pi_{1}^{o r b}(\mathcal{X})$. Hence the torsion-freeness of $\Gamma$ implies 
that the kernel $K$ in diagram 6.5$)$ is trivial. Thus $\Gamma$ is isomorphic to the projective group $\pi_{1}(X)$. In this case the claim follows from Theorem 4.23, see also Proposition 6.24.

In order to conclude the proof we have to show that the case $C=\mathbb{Z}$ cannot occur. In this case since $\Gamma$ has an infinite cyclic normal subgroup, the 3-manifold $N$ is the total space of a Seifert bundle over a 2-dimensional orbifold $\Sigma$; see [28, 45].

If $N$ is closed, then it is finitely covered by a principal $S^{1}$-bundle $\hat{N}$ over a surface $\Sigma_{g}$ of genus $g \geqslant 1$. Since the class of Sasaki groups realizable in a given dimension is closed under passing to finite index subgroups, it suffices to prove that $\pi_{1}(\hat{N})$ is not a Sasaki group realizable in dimension higher than 3. If the $S^{1}$-bundle $\hat{N}$ is trivial, then $\pi_{1}(\hat{N})$ is not a Sasaki group because $\mathrm{b}_{1}\left(\pi_{1}(\hat{N})\right)=2 g+1$. Therefore $\pi_{1}(\hat{N})=\Delta_{g, c}$ must be one of the groups discussed in Remark 6.13, i.e. a Sasaki group that can only be realized in dimension 3 . This settles the case in which $N$ is closed.

If $N$ has non-empty boundary, any cyclic subgroup of $\Gamma$ is generated by a power of the fiber of some Seifert fibration of $N$; see [65, Lemma II.4.8]. In other words, $C$ is generated by $\gamma^{k}$ for some $k \geqslant 1$ where $\gamma$ generates an infinite cyclic subgroup $D \cong \mathbb{Z} \subset \Gamma$. Moreover, $D$ fits in the short exact sequence

$$
0 \longrightarrow D \longrightarrow \Gamma \longrightarrow \pi_{1}^{o r b}(\Sigma) \longrightarrow 0
$$

associated to the Seifert fibration of $N$ over a 2-dimensional orbifold $\Sigma$.

In particular $\pi_{1}^{\text {orb }}(\mathcal{X})$ is a $\mathbb{Z}_{k}$ extension of $\pi_{1}^{\text {orb }}(\Sigma)$ for some $k \geqslant 1$. Thus the LyndonHochschild-Serre spectral sequence for $\mathbb{Z}_{k} \subset \pi_{1}^{o r b}(\mathcal{X})$ gives

$$
H^{*}\left(\pi_{1}^{\text {orb }}(\mathcal{X}) ; \mathbb{R}\right) \cong H^{*}\left(\pi_{1}^{\text {orb }}(\Sigma) ; \mathbb{R}\right) .
$$

Moreover, the isomorphism (6.4) yields $H^{*}\left(\pi_{1}^{\text {orb }}(\Sigma) ; \mathbb{R}\right) \cong H^{*}\left(\pi_{1}(\Sigma) ; \mathbb{R}\right)$ where $\pi_{1}(\Sigma)$ has cohomological dimension 2.

Neither of the above extensions can be trivial because, by Proposition 6.1, one has $[\omega]=\iota^{*}(e)$ where $[\omega] \in H_{o r b}^{2}(\mathcal{X} ; \mathbb{Z})$ is the Euler class of the principal $S^{1}$-orbibundle $\pi: M \longrightarrow \mathcal{X}$ and $e \in H^{2}\left(\pi_{1}^{\text {orb }}(\mathcal{X}) ; \mathbb{Z}\right)$ classifies the associated central extension.

Since $e$ has infinite order it defines a non-trivial real class in $H^{2}\left(\pi_{1}^{\text {orb }}(\mathcal{X}) ; \mathbb{R}\right)$. which, by abuse of notation, we denote by $e$. The isomorphism $H^{*}\left(\pi_{1}^{o r b}(\mathcal{X}) ; \mathbb{R}\right) \cong H^{*}\left(\pi_{1}(\Sigma) ; \mathbb{R}\right)$ yields $\iota^{*}(e)^{2}=[\omega]^{2}=0$ thus, the Kähler orbifold $\mathcal{X}$ must be 2 -dimensional.

This implies that $\operatorname{dim}(M)=3$ contradicting the assumption and concluding the proof.

Remark 6.22. Serre proved in [107] that all finite groups are projective. This explains why $\Gamma$ is assumed to be infinite.

As an immediate consequence of Theorem 6.21 we get a dichotomy of infinite Sasaki groups into those realizable in dimension 3 and all others.

Corollary 6.23. A Sasaki group $\Gamma \in \mathcal{S}_{3}$ is realizable in higher dimensions if and only if it is finite. 


\subsection{Further consequences}

Here we present a collection of results that follow directly from diagram 6.5). We begin with a general observation. Most results in this section will then be special occurrences of the following:

Proposition 6.24. If $\Gamma$ is a torsion-free non-Kähler group with trivial center, then $\Gamma$ is not Sasaki.

Proof. If $\Gamma$ were a Sasaki group it would fit in the following central extension:

$$
0 \longrightarrow C \longrightarrow \Gamma \longrightarrow \pi_{1}^{o r b}(\mathcal{X}) \longrightarrow 0
$$

Since the center of $\Gamma$ is trivial we would have an isomorphism $\Gamma \cong \pi_{1}^{\text {orb }}(\mathcal{X})$. We have seen in Section 6.1 that $\pi_{1}^{\text {orb }}(\mathcal{X})$ surjects on $\pi_{1}(X)$ with kernel generated by elements of finite order. Thus $\Gamma$ would be isomorphic to the Kähler group $\pi_{1}(X)$ by torsion-freeness and this contradicts the assumption.

In [92] Napier and Ramachandran proved that Thompson's group $F$ and its generalizations $F_{n, \infty}$ and $F_{n}$ are not Kähler groups. The analogue of the above statement holds true in the Sasakian setting and is indeed a special instance of Proposition 6.24.

Corollary 6.25. The Thompson groups $F, T$ and $V$ and their generalizations $F_{n, \infty}$ and $F_{n}$ are not Sasaki groups.

In particular, we get Theorem 4.32 as a corollary of Proposition 6.24

Corollary 6.26. Let $\Gamma$ be a lattice in $S O(1, n)$ with $n>2$. Then $\Gamma$ is not a Sasaki group.

It is sometimes possible to combine Lemma 6.3 with the properties of specific classes of groups in order to get restrictions on Sasaki groups. This happens for limit groups, a class of groups that was introduced by Sela in [106]. One can characterize limit groups as the class of groups $\Gamma$ such that for every finite set $S \subset \Gamma$ there is a homomorphism to a free group which is injective on $S$, see [29, Corollary 3.10]. Exploiting the properties of limit groups we prove Proposition 6.28 which is the Sasakian analogue of [77, Theorem 6]. Before proving the result we state the following theorem for future reference.

Theorem 6.27 ([85, 46]). If a finitely presentable group $\Gamma$ fits into an exact sequence

$$
0 \longrightarrow C \longrightarrow \Gamma \longrightarrow Q \longrightarrow 0
$$

with $C$ and $Q$ infinite, and $C$ finitely generated, then the first $\ell^{2}$-Betti number $\beta_{1}(\Gamma)$ of $\Gamma$ vanishes.

We can now prove the following 
Proposition 6.28. A non-abelian limit group is a Sasaki group if and only if it is the fundamental group of a closed orientable surface.

Proof. All surface groups are projective, hence Sasaki.

Conversely, let $\Gamma$ be a limit group which is the fundamental group of a compact Sasakian manifold $M$. Then a quasi-regular structure on $M$ yields the sequence

$$
0 \longrightarrow C \longrightarrow \Gamma \longrightarrow \pi_{1}^{\text {orb }}(X) \longrightarrow 0 \text {. }
$$

Here $C$ cannot be a non-trivial finite group since limit groups are torsion-free. It was proven in [97] that the first $\ell^{2}$-Betti number of non-abelian limit groups is positive. This, together with Theorem 6.27, rules out the instance $C=\mathbb{Z}$. The last case is $C=0$ but this instance cannot occur either. In fact, if $C=0$, then $\Gamma$ is isomorphic to the Kähler group $\pi_{1}(X)$ as a consequence of Lemma 6.3 and torsion-freeness. The claim follows from [77, Theorem 6]. 



\section{Bibliography}

[1] A. Adem, J. Leida and Y. Ruan, Orbifolds and stringy topology, Cambridge Tracts in Mathematics, 171. Cambridge University Press, Cambridge, 2007.

[2] J. Amorós, M. Burger, K. Corlette, D. Kotschick and D. Toledo, Fundamental groups of compact Kähler manifolds, Mathematical Surveys and Monographs, 44. American Mathematical Society, Providence, RI, 1996.

[3] D. Arapura, P. Bressler and M. Ramachandran, On the fundamental group of a compact Kähler manifold, Duke Math. J. 68 (1992), no. 3, 477-488.

[4] M. Aschenbrenner, S. Friedl and H. Wilton, 3-manifold groups, EMS Series of Lectures in Mathematics. European Mathematical Society (EMS), Zürich, 2015.

[5] W. L. Baily Jr., The decomposition theorem for V-manifolds, Amer. J. Math. 78 (1956), 862-888.

[6] W. L. Baily Jr., On the imbedding of $V$-manifolds in projective space, Amer. J. Math. 79 (1957), 403-430.

[7] D. Barden, Simply connected five-manifolds, Ann. of Math. (2) 82 (1965), 365385.

[8] D. E. Blair, Contact manifolds in Riemannian geometry, Lecture Notes in Mathematics, Vol. 509. Springer-Verlag, Berlin-New York, 1976.

[9] D. E. Blair, Riemannian geometry of contact and symplectic manifolds, Progress in Mathematics, 203. Birkhäuser Boston, Inc., Boston, MA, 2010.

[10] I. Biswas, M. Fernández, V. Muñoz and A. Tralle, On formality of Sasakian manifolds, J. Topol. 9 (2016), no. 1, 161-180.

[11] W. M. Boothby and H. C. Wang, On contact manifolds, Ann. of Math. (2) 68 (1958), 721-734.

[12] M. S. Borman, Y. Eliashberg and E. Murphy, Existence and classification of overtwisted contact structures in all dimensions, Acta Math. 215 (2015), no. 2, 281361. 
[13] R. Bott, On a theorem of Lefschetz, Michigan Math. J. 6 (1959), 211-216.

[14] F. Bourgeois, Odd dimensional tori are contact manifolds, Int. Math. Res. Not. (2002) no. 30, 1571-1574.

[15] C. P. Boyer and K. Galicki, Sasakian geometry, Oxford Mathematical Monographs. Oxford University Press, Oxford, 2008.

[16] C. P. Boyer, K. Galicki and J. Kollár, Einstein metrics on spheres, Ann. of Math. (2) 162 (2005), no. 1, 557-580.

[17] C. P. Boyer, K. Galicki and L. Ornea, Constructions in Sasakian geometry, Math. Z. 257 (2007), no. 4, 907-924.

[18] V. Braungardt and D. Kotschick, Einstein metrics and the number of smooth structures on a four-manifold, Topology 44 (2005), no. 3, 641-659.

[19] K. S. Brown, Cohomology of groups, Graduate Texts in Mathematics, 87. Springer-Verlag, New York-Berlin, 1982.

[20] F. Campana, Remarques sur les groupes de Kähler nilpotents, C. R. Acad. Sci. Paris Sér. I Math. 317 (1993), no. 8, 777-780.

[21] F. Campana, Orbifoldes géométriques spéciales et classification biméromorphe des variétés kählériennes compactes, J. Inst. Math. Jussieu 10 (2011), no. 4, 809934.

[22] F. Campana, Quotients résolubles ou nilpotents des groupes de Kähler orbifoldes, Manuscripta Math. 135 (2011), no. 1-2, 117-150.

[23] B. Cappelletti-Montano, A. De Nicola and J. C. Marrero, Sasakian nilmanifolds, Int. Math. Res. Not. IMRN (2015) no. 15, 6648-6660.

[24] B. Cappelletti-Montano, A. De Nicola, J. C. Marrero and I. Yudin, A non-Sasakian Lefschetz K-contact manifold of Tievsky type, Proc. Amer. Math. Soc. 144 (2016), no. $12,5341-5350$.

[25] B. Cappelletti-Montano, A. De Nicola and I. Yudin, Hard Lefschetz theorem for Sasakian manifolds, J. Differential Geom. 101 (2015), no. 1, 47-66.

[26] J. A. Carlson and D. Toledo, Rigidity of harmonic maps of maximum rank, J. Geom. Anal. 3 (1993), no. 2, 99-140.

[27] J. A. Carlson and D. Toledo, Quadratic presentations and nilpotent Kähler groups, J. Geom. Anal. 5 (1995), no. 3, 359-377.

[28] A. Casson and D. Jungreis, Convergence groups and Seifert fibered 3-manifolds, Invent. Math. 118 (1994), no. 3, 44-456. 
[29] C. Champetier and V. Guirardel, Limit groups as limits of free groups, Israel J. Math. 146 (2005), 1-75.

[30] X. Chen, On the fundamental groups of compact Sasakian manifolds, Math. Res. Lett. 20 (2013), no. 1, 27-39.

[31] W. Chen and Y. Ruan, Orbifold Gromov-Witten theory, Orbifolds in mathematics and physics (Madison, WI, 2001), 25-85, Contemp. Math., 310, Amer. Math. Soc., Providence, RI, 2002.

[32] P. C. Craighero and R. Gattazzo, Quintic surfaces of $\mathbf{P}^{3}$ having a nonsingular model with $q=p_{g}=0, P_{2} \neq 0$, Rend. Sem. Mat. Univ. Padova 91 (1994), 187-198.

[33] P. Deligne, P. Griffiths, J. Morgan and D. Sullivan, Real homotopy theory of Kähler manifolds, Invent. Math. 29 (1975), no. 3, 245-274.

[34] C. Delorme, Espaces projectifs anisotropes, Bull. Soc. Math. France 103 (1975), no. 2, 203-223.

[35] A. Dimca and A. Suciu, Which 3-manifold groups are Kähler groups?, J. Eur. Math. Soc. (JEMS) 11 (2009), no. 3, 521-528.

[36] I. Dolgachev, Weighted projective varieties, Group actions and vector fields (Vancouver, B.C., 1981), 34-71, Lecture Notes in Math., 956, Springer, Berlin, 1982.

[37] I. Dolgachev and C. Werner, A simply connected numerical Godeaux surface with ample canonical class, J. Algebraic Geom. 8 (1999), no. 4, 737-764.

[38] W. Ebeling, An example of two homeomorphic, nondiffeomorphic complete intersection surfaces, Invent. Math. 99 (1990), no. 3, 651-654.

[39] Y. Eliashberg, Classification of overtwisted contact structures on 3-manifolds, Invent. Math. 98 (1989), no. 3, 623-637.

[40] Y. Eliashberg, Filling by holomorphic discs and its applications, Geometry of lowdimensional manifolds, 2 (Durham, 1989), 45-67, London Math. Soc. Lecture Note Ser., 151, Cambridge Univ. Press, Cambridge, 1990.

[41] A. El Kacimi-Alaoui, Opérateurs transversalement elliptiques sur un feuilletage riemannien et applications, Compositio Math. 73 (1990), no. 1, 57-106.

[42] A. El Kacimi-Alaoui and G. Hector, Décomposition de Hodge basique pour un feuilletage riemannien, Ann. Inst. Fourier (Grenoble) 36 (1986), no. 3, 207-227.

[43] A. El Kacimi-Alaoui and M. Nicolau, On the topological invariance of the basic cohomology, Math. Ann. 295 (1993), no. 4, 627-634. 
[44] Y. Félix, J. Oprea and D. Tanré, Algebraic models in geometry, Oxford Graduate Texts in Mathematics, 17. Oxford University Press, Oxford, 2008.

[45] D. Gabai, Convergence groups are Fuchsian groups, Ann. of Math. (2) 136 (1992), no. 3, 447-510.

[46] D. Gaboriau, Invariants $\ell^{2}$ de relations d'équivalence et de groupes, Publ. Math. Inst. Hautes Études Sci. No. 95 (2002), 93-150.

[47] H. Geiges, Contact structures on 1-connected 5-manifolds, Mathematika 38 (1991), no. 2, 303-311.

[48] H. Geiges, Normal contact structures on 3-manifolds, Tohoku Math. J. (2) 49 (1997), no. 3, 415-422.

[49] O. Goertsches, H. Nozawa and D. Töben, Rigidity and vanishing of basic Dolbeault cohomology of Sasakian manifolds, J. Symplectic Geom. 14 (2016), no. 1, 31-70.

[50] R. E. Gompf, A new construction of symplectic manifolds, Ann. of Math. (2) 142 (1995), no. 3, 527-595.

[51] R. Goto, Calabi-Yau structures and Einstein-Sasakian structures on crepant resolutions of isolated singularities, J. Math. Soc. Japan 64 (2012), no. 3, 1005-1052.

[52] J. W. Gray, Some global properties of contact structures, Ann. of Math. (2) 69 (1959), 421-450.

[53] W. Greub, S. Halperin and R. Vanstone, Connections, curvature, and cohomology. Vol. II: Lie groups, principal bundles, and characteristic classes, Pure and Applied Mathematics, Vol. 47-II. Academic Press, New York-London, 1973.

[54] M. Gromov, Sur le groupe fondamental d'une variété kählérienne, C. R. Acad. Sci. Paris Sér. I Math. 308 (1989), no. 3, 67-70.

[55] A. Haefliger, Variétés feuilletées, Ann. Scuola Norm. Sup. Pisa Cl. Sci. (3) 16 (1962), 367-397.

[56] A. Haefliger, Homotopy and integrability, 1971 Manifolds-Amsterdam 1970 (Proc. Nuffic Summer School) 133-163 Lecture Notes in Mathematics, 197 Springer, Berlin

[57] A. Haefliger and E. Salem, Actions of Tori on Orbifolds, Ann. Glob. Anal. Geom. 9 (1991), 37-59.

[58] S. Halperin, Lectures on minimal models, Mém. Soc. Math. France (N.S.) No. 9-10 (1983). 
[59] M. J. D. Hamilton, Inequivalent contact structures on Boothby-Wang fivemanifolds, Math. Z. 274 (2013), no. 3-4, 719-743.

[60] F. Hirzebruch, Topological methods in algebraic geometry, Classics in Mathematics. Springer-Verlag, Berlin, 1995.

[61] E. Horikawa, Algebraic surfaces of general type with small $c_{1}^{2}$. I, Ann. of Math. (2) 104 (1976), no. 2, 357-387.

[62] S. Ianuş, Sulle varietà di Cauchy-Riemann, Rend. Accad. Sci. Fis. Mat. Napoli (4) 39 (1972), 191-195.

[63] M. Itoh, Odd-dimensional tori and contact structure, Proc. Japan Acad. Ser. A Math. Sci. 73 (1997), no. 4, 58-59.

[64] W. H. Jaco, Finitely presented subgroups of three-manifold groups, Invent. Math. 13 (1971), 335-346.

[65] W. H. Jaco and P. B. Shalen, Seifert fibered spaces in 3-manifolds, Mem. Amer. Math. Soc. 21 (1979).

[66] F. E. A. Johnson and E. G. Rees, On the fundamental group of a complex algebraic manifold, Bull. London Math. Soc. 19 (1987) 463-466.

[67] P. E. Jupp, Classification of certain 6-manifolds, Proc. Cambridge Philos. Soc. 73 (1973), 293-300.

[68] H. Kasuya, Cohomologies of Sasakian groups and Sasakian solvmanifolds, Ann. Mat. Pura Appl. (4) 195 (2016), no. 5, 1713-1719.

[69] H. Kasuya, Mixed Hodge structures and Sullivan's minimal models of Sasakian manifolds, Ann. Inst. Fourier (Grenoble) 67 (2017), no. 6, 2533-2546.

[70] S. Kobayashi, Principal fibre bundles with the 1-dimensional toroidal group, Tohoku Math. J. (2) 8 (1956), 29-45.

[71] K. Kodaira and D. C. Spencer, On a theorem of Lefschetz and the lemma of Enriques-Severi-Zariski, Proc. Nat. Acad. Sci. U.S.A. 39 (1953), 1273-1278.

[72] J. Kollár, Shafarevich maps and plurigenera of algebraic varieties, Invent. Math. 113 (1993), no. 1, 177-215.

[73] J. Kollár, Seifert $G_{m}$-bundles, preprint, arXiv:math/0404386v2 (2004).

[74] J. Kollár, Einstein metrics on five-dimensional Seifert bundles, J. Geom. Anal. 15 (2005), no. 3, 445-476.

[75] D. Kotschick, All fundamental groups are almost complex, Bull. London Math. Soc. 24 (1992), no. 4, 377-378. 
[76] D. Kotschick, All fundamental groups are almost contact, Bull. London Math. Soc. 24 (1992), no. 5, 493-494.

[77] D. Kotschick, The deficiencies of Kähler groups, J. Topol. 5 (2012), no. 3, 639650 .

[78] D. Kotschick, Three-manifolds and Kähler groups, Ann. Inst. Fourier (Grenoble) 62 (2012), no. 3, 1081-1090.

[79] D. Kotschick, Kählerian three-manifold groups, Math. Res. Lett. 20 (2013), no. 3, 521-525.

[80] M. Kreck, Surgery and duality, Ann. of Math. (2) 149 (1999), no. 3, 707-754.

[81] S. Lang, Topics in cohomology of groups, Translated from the 1967 French original by the author. Chapter X based on letters written by J. Tate. Lecture Notes in Mathematics, 1625. Springer-Verlag, Berlin, 1996.

[82] J. K. Leida, Orbifolds and stable equivariant homotopy groups, Thesis (Ph.D.)The University of Wisconsin - Madison 2006.

[83] E. Lerman, Contact fiber bundles, J. Geom. Phys. 49 (2004), no. 1, 52-66.

[84] A. S. Libgober and J. W. Wood, Differentiable structures on complete intersections. I, Topology 21 (1982), no. 4, 469-482.

[85] W. Lück, Hilbert modules and modules over finite von Neumann algebras and applications to $L^{2}$-invariants, Math. Ann. 309 (1997), no. 2, 247-285.

[86] J. Milnor, A unique decomposition theorem for 3-manifolds, Amer. J. Math. 84 $19621-7$.

[87] J. Milnor, Singular points of complex hypersurfaces, Annals of Mathematics Studies, No. 61 Princeton University Press, Princeton, N.J.; University of Tokyo Press, Tokyo 1968.

[88] I. Moerdijk and J. Mrčun, Introduction to foliations and Lie groupoids, Cambridge Studies in Advanced Mathematics, 91. Cambridge University Press, Cambridge, 2003.

[89] I. Moerdijk and D. A. Pronk, Orbifolds, sheaves and groupoids, K-Theory 12 (1997), no. 1, 3-21.

[90] P. Molino, Riemannian foliations, Progress in Mathematics, 73. Birkhäuser Boston, Inc., Boston, MA, 1988.

[91] S. Nagami, Existence of Spin structures on double branched covering spaces over four-manifolds, Osaka J. Math. 37 (2000), no. 2, 425-440. 
[92] T. Napier and M. Ramachandran, Thompson's group F is not Kähler, Contemp. Math., 394, Amer. Math. Soc., Providence, RI, 2006.

[93] K. Niederkrüger, The plastikstufe - a generalization of the overtwisted disk to higher dimensions, Algebr. Geom. Topol. 6 (2006), 2473-2508.

[94] K. Niederkrüger and F. Pasquotto, Resolution of symplectic cyclic orbifold singularities, J. Symplectic Geom. 7 (2009), no. 3, 337-355.

[95] H. Nozawa, Deformation of Sasakian metrics, Trans. Amer. Math. Soc. 366 (2014), no. 5, 2737-2771.

[96] M. Okumura, On contact metric immersion, Kōdai Math. Sem. Rep. 20 (1968), 389-409.

[97] M. Pichot, Semi-continuity of the first $\ell^{2}$-Betti number on the space of finitely generated groups, Comment. Math. Helv. 81 (2006), no. 3, 643-652.

[98] D. Prill, Local classification of quotients of complex manifolds by discontinuous groups, Duke Math. J. 34 (1967), 375-386.

[99] J. Rana, J. Tevelev and G. Urzúa, The Craighero-Gattazzo surface is simply connected, Compos. Math. 153 (2017), no. 3, 557-585.

[100] P. Raźny, Invariance of basic Hodge numbers under deformations of Sasakian manifolds, preprint, arXiv:1908.11107 (2019).

[101] P. Rukimbira, Some remarks on R-contact flows, Ann. Global Anal. Geom. 11 (1993), no. 2, 165-171.

[102] S. Sasaki, On differentiable manifolds with certain structures which are closely related to almost contact structure. I, Tohoku Math. J. (2) 12 (1960), 459-476.

[103] S. Sasaki and Y. Hatakeyama, On differentiable manifolds with contact metric structures, J. Math. Soc. Japan 14 (1962), 249-271.

[104] I. Satake, On a generalization of the notion of manifold, Proc. Nat. Acad. Sci. U.S.A. 42 (1956), 359-363.

[105] I. Satake, The Gauss-Bonnet theorem for V-manifolds, J. Math. Soc. Japan 9 (1957), 464-492.

[106] Z. Sela, Diophantine geometry over groups. I. Makanin-Razborov diagrams, Publ. Math. Inst. Hautes Études Sci. No. 93 (2001), 31-105.

[107] J.-P. Serre, Sur la topologie des variétés algébriques en caractéristique p, (1958) Symposium internacional de topología algebraica International symposium on algebraic topology pp. 24-53 Universidad Nacional Autónoma de México and UNESCO, Mexico City. 
[108] J.-P. Serre, Corps locaux, Publications de l'Institut de Mathématique de l’Université de Nancago, VIII Actualités Sci. Indust., No. 1296. Hermann, Paris 1962.

[109] S. Tachibana, On harmonic tensors in compact Sasakian spaces, Tohoku Math. J. (2) 17 (1965), 271-284.

[110] S. Tanno, Harmonic forms and Betti numbers of certain contact Riemannian manifolds, J. Math. Soc. Japan 19 (1967) 308-316.

[111] S. Tanno, Variational problems on contact Riemannian manifolds, Trans. Amer. Math. Soc. 314 (1989), no. 1, 349-379.

[112] C. H. Taubes, The existence of anti-self-dual conformal structures, J. Differential Geom. 36 (1992), no. 1, 163-253.

[113] A. M. Tievsky, Analogues of Kähler geometry on Sasakian manifolds, Thesis (Ph.D.)-Massachusetts Institute of Technology. 2008.

[114] C. Traving, Zur Diffeomorphieklassifikation vollständiger Durchschnitte, Diplomarbeit, Mainz (1985). Available under: https://www.maths.ed.ac.uk/ v1ranick/papers/traving.pdf

[115] A. W. Wadsley, Geodesic foliations by circles, J. Differential Geometry 10 (1975), no. 4, 541-549.

[116] C. T. C. Wall, Classification problems in differential topology. V. On certain 6manifolds, Invent. Math. 1 (1966), 355-374.

[117] J. Wang and J. Du, Geometrical realization of low-dimensional complete intersections, Chin. Ann. Math. Ser. B 37 (2016), no. 4, 523-532. 



\section{Eidesstattliche Versicherung \\ (Siehe Promotionsordnung vom 12.07.11, § 8, Abs. 2 Pkt. .5.)}

Hiermit erkläre ich an Eidesstatt, dass die Dissertation von mir selbstständig, ohne unerlaubte Beihilfe angefertigt ist.

Placini, Giovanni

Name, Vorname

München, 06.08.2020

Giovanni Placini

Ort, Datum

Unterschrift Doktorand 